\title{
Digital-to-Time Converters for spur correction \\ in Digital Frequency Synthesis
}

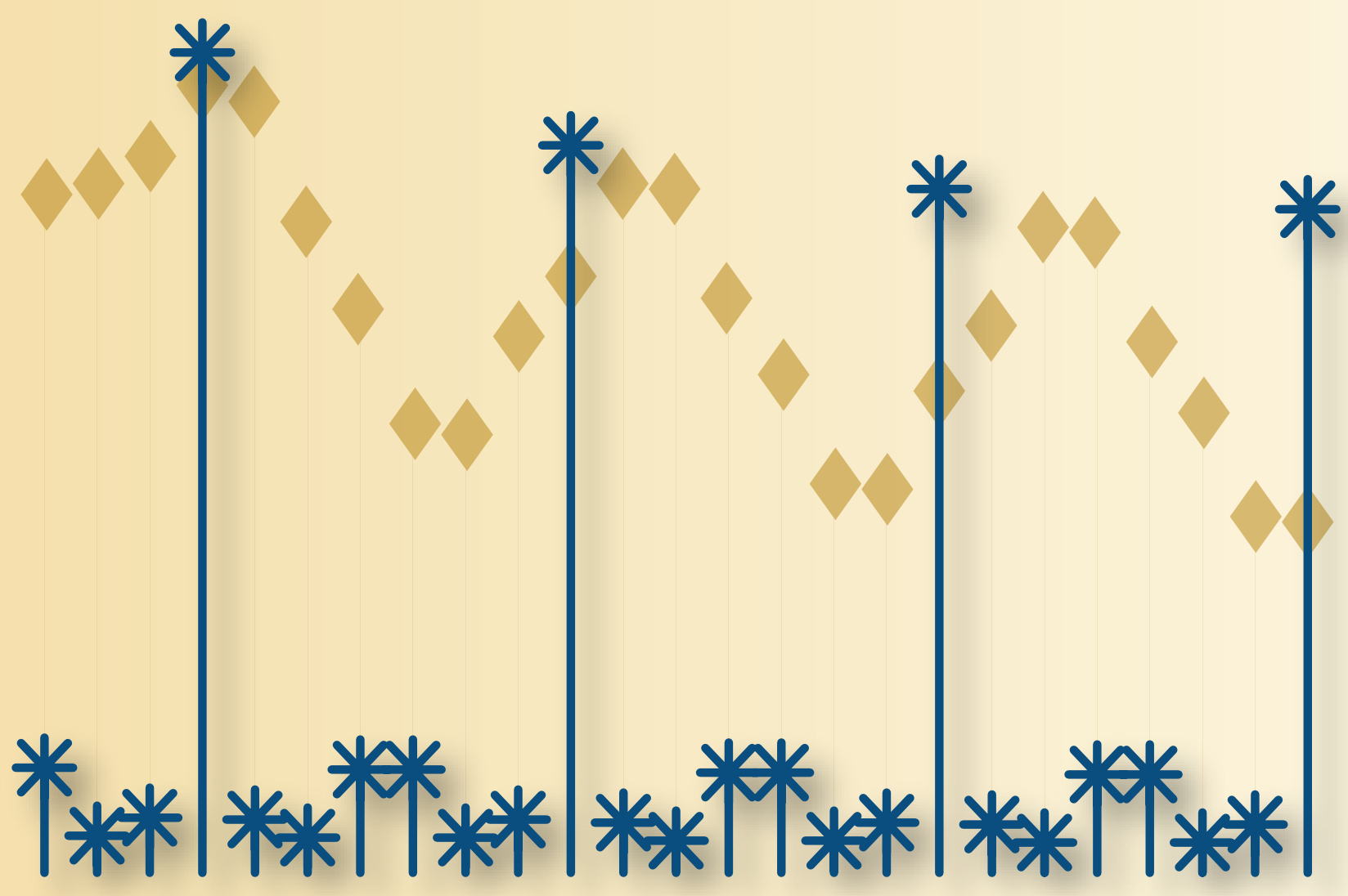

Claudia Palattella 


\section{Digital-to-Time Converters for spur correction in Digital Frequency Synthesis}


Members of the dissertation committee:

Prof. dr. J.N. Kok University of Twente (chairperson/secretary)

Prof. dr. ir. B. Nauta University of Twente (supervisor)

Dr. ing. E.A.M. Klumperink University of Twente (co-supervisor)

Prof. dr. ir. F.E. van Vliet University of Twente

Prof. dr. J.A. Ferreira University of Twente

Prof. dr. ir. P.G.M. Baltus Eindhoven University of Technology

Prof. dr. P.P. Sotiriadis National Technical University of Athens

Copyright (C) 2020 by Claudia Palattella, Enschede, The Netherlands.

Printed by Gildeprint Drukkerijen, Enschede, The Netherlands.

ISBN 978-90-365-5015-4

DOI $\quad 10.3990 / 1.978903655015$

https:/ / doi.org/10.3990/1.9789036550154 


\title{
DigITAL-TO-TIME CONVERTERS FOR SPUR CORRECTION IN Digital FREQUENCY SYNTHESIS
}

\section{PROEFSCHRIFT}

\author{
ter verkrijging van \\ de graad van doctor aan de Universiteit Twente, \\ op gezag van de rector magnificus, \\ prof. dr. T.T.M. Palstra, \\ volgens besluit van het College voor Promoties \\ in het openbaar te verdedigen \\ op vrijdag 8 juli 2020 om 16.45 uur
}

door

Claudia Palattella

geboren op 19 juni 1983

te Putignano (BA), Italy 
Dit proefschrift is goedgekeurd door:

Prof. dr. ir. B. Nauta (promotor)

Dr. ing. E.A.M. Klumperink (co-promotor) 
Ai miei genitori 



\section{Abstract}

The demand for wireless communication, mobile computing and multifunctional portable electronics has driven System-on-Chip (SoC) solutions, with increasing level of integration. SoCs are heterogeneous systems, where many functional blocks running at difference clock frequencies coexist on the same chip. This requires an on-chip clock source that must be able to generate a wide range of frequencies in a flexible way, so that they can be changed quickly to accommodate the sophisticated communication activities occurring among the blocks.

Direct Digital Synthesizers (DDSs), known from the 1970s[1-3], have gained a renewed interest since they offer wide frequency range and greater flexibility than commonly used Phase-Locked Loop (PLL) solutions, as well as compatibility with digital design flows. Direct Digital Synthesizers using a D/A converter to generate the output sine-wave ("sinewave DFC") typically achieve lower maximum output frequency than that of commensurate PLLs, but they cover the full frequency range, from zero to the maximum one, with extremely fine frequency resolution, and instantaneous, digitally programmed frequency and phase switching. When producing different frequencies in SoCs, a key benefit of DDSs is that a single simple integer-N PLL is needed to produce a (unique) high-frequency system clock, to control multiple DDS blocks. Those blocks can generate all other frequencies required in the system, in a flexible, digitally programmable way. This allows an optimal design of the integer-N PLL, improving significantly the overall performance of frequency generation, including the minimization of the phase noise.

In many clocking applications, a sinewave is not necessary, and a pulsed clock can be used instead. To address such needs, the concept of PulseOutput Digital-to-Frequency Converter (DFC)[4-6] has been the starting point for the research developed in this thesis. A key limitation of this architecture is deterministic jitter, due to periodic phase errors produced by its digital core [7]. The research described in this thesis focuses on reducing the deterministic jitter exploiting Digital-to-Time Converters (DTCs).

DTC based on the constant-slope principle have been proven to provide 10-bit accuracy and they are a solid starting point to design a DTC with good properties for deterministic jitter compensation, especially high resolution and high linearity, as well as low thermally induced jitter. In this thesis, the constant-slope principle has been extended to make it suitable for 
$\mathrm{GHz}$ operating frequencies, leading to the design of a new time-interleaved DTC with fully differential output, operating on both clock edges.

To characterize the DTC in terms of resolution and linearity, the tiny DTC delay steps ( $100 \mathrm{fs}$ ) should be measured with sufficient precision. In this thesis, we propose a method to measure DTC delay steps with unprecedented time resolution, as low as femto-seconds, with common lab equipment. The method exploits digital phase modulation to produce spurs related to the size of the DTC delay-steps.

To correct the deterministic jitter of a Pulse-Output DFC, we propose in this thesis a spur correction with the aforementioned developed and studied DTC. The system analysis developed in this work shows that a DTC with 11-bit resolution and a few LSB INL is capable to push down the sub-harmonics spurs of the Pulse-Output DFC to $-60 \mathrm{dBc}$. A mathematical model is derived that (1) predicts the strength of all the sub-harmonic spurs and (2) relates the maximum spur to the DTC Integral Non-Linearity (INL) in a simple, closed-form expression. This allows the designer to derive DTC INL requirements given a Spurious-Free Dynamic Range (SFDR) target.

A fully-integrated prototype of a Pulse-Output DFC with DTC-based spur correction has been implemented in $1.2 \mathrm{~V} 65 \mathrm{~nm}$ CMOS technology. Measurements demonstrate basic functionality but do not achieve the target $-60 \mathrm{dBc}$ spur level. Worst case spurs are $<-44 \mathrm{dBc}$ for an arbitrarily programmable frequency up to $500 \mathrm{MHz}$, with a power consumption of $51 \mathrm{~mW}$. The DTC spur correction is limited by sub-optimal layout as well as by the DTC INL and likely timing violation at the interface between the DFC core and the DTC.

Overall, it is shown that the spur-reduction technique based on DTC correction is promising for flexible digitally programmable frequency synthesis, but more work is needed to improve high-speed DTC linearity, deal with PVT variations and reduce power. 


\section{Samenvatting}

De vraag naar draadloze communicatie, mobiele rekenkracht en multifunctionele draagbare elektronica heeft geleid tot System-on-Chip (SoC) -oplossingen, met een toenemende mate van integratie. SoCs zijn heterogene systemen, waarbij veel functionele blokken die op verschillende klokfrequenties lopen naast elkaar op dezelfde chip bestaan. Dit vereist een on-chip klokbron die op flexibele wijze een breed scala aan frequenties moet kunnen genereren, zodat ze snel kunnen worden gewijzigd om tegemoet te komen aan de geavanceerde communicatieactiviteiten tussen de blokken.

Direct Digital Synthesizers (DDSs), bekend uit de jaren 70 [1-3], staan opnieuw in de belangstelling omdat ze een breed frequentiebereik en grotere flexibiliteit bieden dan veelgebruikte Phase-Locked Loop (PLL)oplossingen en goed passen in een digitaal ontwerpproces. Direct digital synthesizers die een D/A-omzetter gebruiken om de uitgangssinusgolf te genereren ("sinewave DFC "), bereiken doorgaans een lagere maximale uitgangsfrequentie dan die van vergelijkbare PLL's, maar ze bestrijken wel het volledige frequentiebereik, van nul tot het maximum, met extreem fijne frequentieresolutie en directe, digitaal gestuurde frequentie- en faseomschakeling. Bij het maken van verschillende frequenties in SoCs is een belangrijk voordeel van DDSen is dat er een enkele eenvoudige integer-N PLL nodig is om een (unieke) hoogfrequente systeemklok te produceren, die meerdere DDS-blokken aan kan sturen. Deze blokken kunnen op een flexibele, digitaal programmeerbare manier alle andere in het systeem benodigde frequenties genereren. Dit maakt een optimaal ontwerp van de integer-N PLL mogelijk, waardoor de algehele prestatie van frequentieopwekking aanzienlijk wordt verbeterd en faseruis wordt geminimaliseerd.

In veel kloktoepassingen is een sinusgolf niet nodig en kan in plaats daarvan een pulsvormige klok worden gebruikt. Om in dergelijke behoeften te voorzien, is het concept van Pulse-Output Digital-to-Frequency Converter (DFC) [4-6] het uitgangspunt geweest voor het onderzoek in dit proefschrift. Een belangrijke beperking van deze architectuur is deterministische jitter, als gevolg van periodieke fasefouten die worden veroorzaakt door de digitale kern van het systeem [7]. Het in dit proefschrift beschreven onderzoek richt zich op het verminderen van de deterministische jitter door middel van Digital-to-Time Converters (DTCs).

Het is bewezen dat DTCs op basis van het constant-slope principe 
10-bit nauwkeurigheid bieden en ze vormen een goed startpunt voor het ontwerpen van een DTC met goede eigenschappen voor deterministische jittercompensatie, met name de hoge resolutie en hoge lineariteit en lage jitter van thermische oorsprong. In dit proefschrift is het constant-slope principe uitgebreid om het geschikt te maken voor $\mathrm{GHz}$-frequenties, wat heeft geleid tot het ontwerp van een nieuwe time-interleaved DTC met differentële output, die op beide klokflanken werkt.

Om de lineariteit en resolutie van de DTC te karakteriseren, moeten de kleine DTC-vertragingsstappen ( $100 \mathrm{fs}$ ) met voldoende precisie worden gemeten. In dit proefschrift stellen we een methode voor om DTCvertragingsstappen te meten met een voorheen ongekende tijdsresolutie tot in de femto-seconden, met standaard meetapparatuur. De methode maakt gebruik van digitale fasemodulatie om frequentiecomponenten te produceren die verband houden met de grootte van de DTC-vertragingsstappen.

Om de deterministische jitter van een Pulse-Output DFC te corrigeren, introduceren we in dit proefschrift een correctie op basis van frequentiecomponenten, met de hier ontwikkelde en bestudeerde DTC. De systeemanalyse in dit onderzoek laat zien dat een DTC met 11-bit resolutie en een paar LSB INL in staat is om de ongewenste subharmonische frequenties van de Pulse-Output DFC naar $-60 \mathrm{dBc}$ te brengen. Een wiskundig model is afgeleid dat (1) de sterkte van alle subharmonischen voorspelt en (2) de maximale subharmonische component relateert aan de DTC Integral Non-Linearity (INL) in een eenvoudige vergelijking. Hierdoor kan een ontwerper DTC INL-vereisten afleiden op basis van een beoogde Spurious-Free Dynamic Range (SFDR).

Een volledig geïntegreerd prototype van een Pulse-Output DFC met op DTC gebaseerde spectrumcorrectie is geïmplementeerd in $1.2 \mathrm{~V} 65 \mathrm{~nm}$ CMOS-technologie. Metingen tonen basisfunctionaliteit aan, maar halen niet het beoogde doel van $-60 \mathrm{dBc}$ subharmonischen. In het ergste geval zijn de spurs $<-44 \mathrm{dBc}$ voor een willekeurig programmeerbare frequentie tot $500 \mathrm{MHz}$, met een energieverbruik van $51 \mathrm{~mW}$. De DTC-correctie wordt beperkt door een niet-optimale lay-out evenals door de DTC INL en waarschijnlijk door timing-problemen op de interface tussen de DFC-kern en de DTC.

Over het geheel genomen is aangetoond dat de spectrumcorrectie op basis van DTC-correctie veelbelovend is voor flexibele digitaal programmeerbare frequentiesynthese, maar er zijn vervolgstappen nodig om de DTC-lineariteit op deze hoge snelheden te verbeteren, PVT-variaties aan te pakken en het vermogensverbruik te verminderen. 


\section{List of Acronyms}

CF Charge for falling edge

CML Current-Mode Logic

CR Charge for rising edge

DAC Digital-to-Analog Converter

DDL Digital Delay Line

DDS Direct Digital Synthesizer

DFC Digital-to-Frequency Converter

DFT Discrete Fourier Transform

DLL Delay-Locked Loop

DNL Differential Nonlinearity

DPC Digital-to-Period Converter

DTC Digital-to-Time Converter

DVFS Dynamical voltage and frequency scaling

DW Delay Word

ENOB Effective Number of Bits

FCW Frequency Control Word

FoM Figure-of-Merit

GRR Grand Repetition Rate

IC Integrated Circuit

INL Integral Non-Linearity
LO Local Oscillator

LVT Low-Voltage

MMD Multi-Modulus Divider

p.d.f. probability density function

p.m.f. probability mass function

PLL Phase-Locked Loop

PSD Power Spectral Density

PVT Process-Voltage-Temperature

RSCE Reverse Short-Channel Effect

RST Reset

SA Split-Apart

SFDR Spurious-Free Dynamic Range

SINAD Signal-to-Noise And Distortion ratio

SoC System-on-Chip

TDC Time-to-Digital Converter

VCO Voltage-Controlled Oscillator

VTC Voltage-to-Time Converter

ZCD Zero Crossing Detector 


\section{Contents}

Abstract vii

Samenvatting $\quad i x$

List of Acronyms $x i$

1 Introduction $\quad 1$

1.1 Deterministic jitter compensation 3

1.2 Research Goals 5

$\begin{array}{lll}1.3 & \text { Thesis Outline } & 7\end{array}$

2 State-of-the-art DTCs 9

2.1 Constant-slope principle 10

2.2 Architectures 13

2.3 DTC benchmarking 21

2.4 Conclusions 21

3 A Sensitive Method to Measure the INL of a DTC 23

3.1 Introduction 23

3.2 Proposed Method 26

3.2.1 The concept 26

3.2.2 Analysis 26

3.2.3 Sensitivity and Resolution $\quad 27$

3.2.4 Algorithm 29

3.3 Simulations 30

3.4 Measurements 32

3.5 Conclusion 34

4 Pulse-Output DFCs: reducing spurs with a DTC $\quad 35$

4.1 Introduction 35

4.2 Brief overview on DPCs and DFCs 37

4.3 Proposed Architecture 39

4.3.1 Pulse-Output DFC 39

4.3.2 Digital-to-Time Converter (DTC) for edge retiming 39

4.3.3 Pushing down spur levels with a DTC 41

4.3.4 DTC-based Pulse-Output DFC 43

4.4 Output spectrum in a DTC-based DFC 46 
4.4.1 Effect of DTC quantization error

4.4.2 Worst-case spur: closed-form estimate 49

4.4.3 Spectrum of DFCs versus DPCs 50

4.4.4 Effect of DTC impairments on the DFC spectrum 53

4.4.5 Worst spur in presence of INL 56

$\begin{array}{ll}4.5 \text { Conclusion } & 58\end{array}$

5 Integrated DFC Prototype: UTDUCK 61

5.1 Top-level architecture $\quad 61$

5.2 Four-phase clock generator 62

5.3 Digital-to-Time Converter 63

5.3.1 DTC Design Challenges 63

5.3.2 Coarse DTC 65

5.3.3 Fine DTC: detailed view 66

5.3.4 State Diagram 68

5.3.5 Complementary Current Sources $\quad 72$

$\begin{array}{ll}\text { 5.3.6 Capacitive DAC } & 74\end{array}$

5.3.7 Zero Crossing Detector $\quad 78$

$\begin{array}{lll}5.4 & \text { Final stages } & 78\end{array}$

5.5 Expected performance and limitations $\quad 79$

5.5.1 Time constraints for the time-interleaved DTC 79

5.5.2 Output jitter 83

$\begin{array}{lll}5.6 & \text { Simulations } & 89\end{array}$

5.7 Measurements $\quad 95$

5.7.1 Additional circuit for measurement 96

5.7.2 Calibration 98

5.7.3 Measurements on the DTC 100

5.7.4 Measurements in DFC mode 106

5.7.5 Maximum spur 108

5.7.6 Phase noise measurements 109

$\begin{array}{lll}\text { 5.7.7 Speed measurements } & 112\end{array}$

$\begin{array}{ll}\text { 5.7.8 Power breakdown } & 116\end{array}$

5.8 Benchmarking 118

$\begin{array}{ll}5.9 \text { Conclusions } & 121\end{array}$

6 Conclusions \& Recommendations 123

6.1 Summary \& Conclusions 123

6.2 Original contributions $\quad 125$

$\begin{array}{ll}6.3 \text { Recommendations } & 126\end{array}$

A Implementation details $\quad 129$

A.1 Capacitive DAC 129

A.2 Digital front-end 133

$\begin{array}{ll}\text { A.3 Calibration } & 133\end{array}$

B Mathematical derivations for the analysis in chapter $4 \quad 139$ 
B.1 Derivation of (4.13) and (4.14) 139

B.2 Proof of the approximation (4.18) 140

B.3 Expected value of INL 142

C Sampled noise power $\quad 147$

C.1 Capacitive DAC 147

$\begin{array}{lll}\text { C.2 Reset switch } \mathrm{RST}_{z} & 149\end{array}$

D Verilog code for the digital design of the front-end 151

$\begin{array}{ll}\text { Acknowledgments } & 161\end{array}$

$\begin{array}{ll}\text { Bibliography } & 165\end{array}$

List of publications $\quad 171$

$\begin{array}{ll}\text { Peer-reviewed } & 171\end{array}$ 


\section{CHAPTER}

\section{Introduction}

Portability and connectivity are nowadays essential features of all kinds of smart devices. We are accustomed to light-weighted devices which can be easily carried around and are always connected to the Internet (Internet of Things, or IoT), to the point that they have changed our lifestyle and created new needs. These factors together with the declining prices of electronics, due to technological advancements and increasing level of integration, are boosting the portable electronics global market, which is expected to grow substantially over the period 2017 - 2022 (Fig. 1.1) [8, 9].

From the perspective of Integrated Circuit (IC) design, innovation in the area of clock generation plays an important role in facing the design challenges related to smart phones and IoT. As the device sizes progressively shrink, more features are incorporated in a single chip, leading to Systemon-Chip (SoC) applications. The coexistence of many functional blocks running at different clock frequencies needs a flexible clock source able not only to provide the required frequencies but also to change them quickly, in order to allow fast communication when needed and slower when the

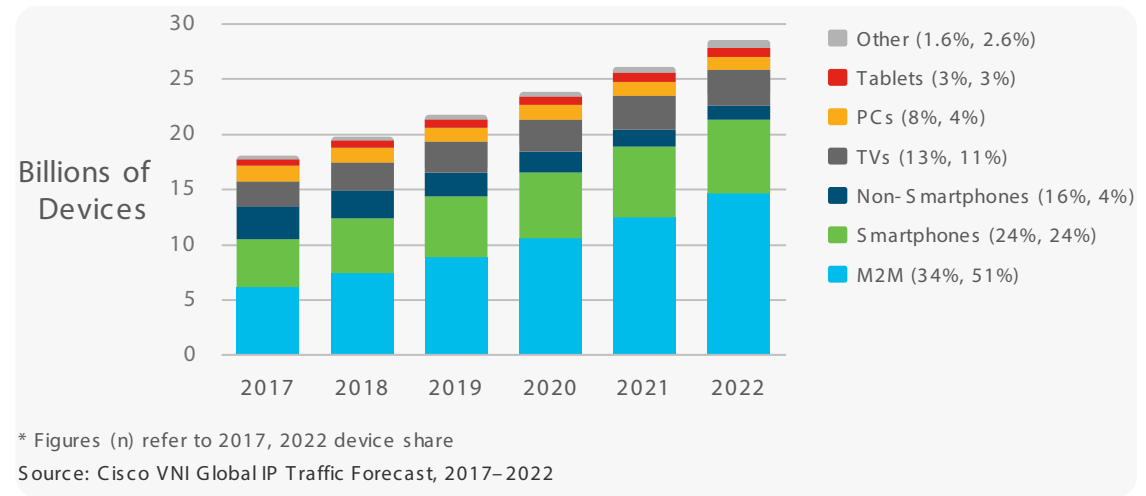

Figure 1.1: Global Mobile Devices and Connections Growth. [8] 


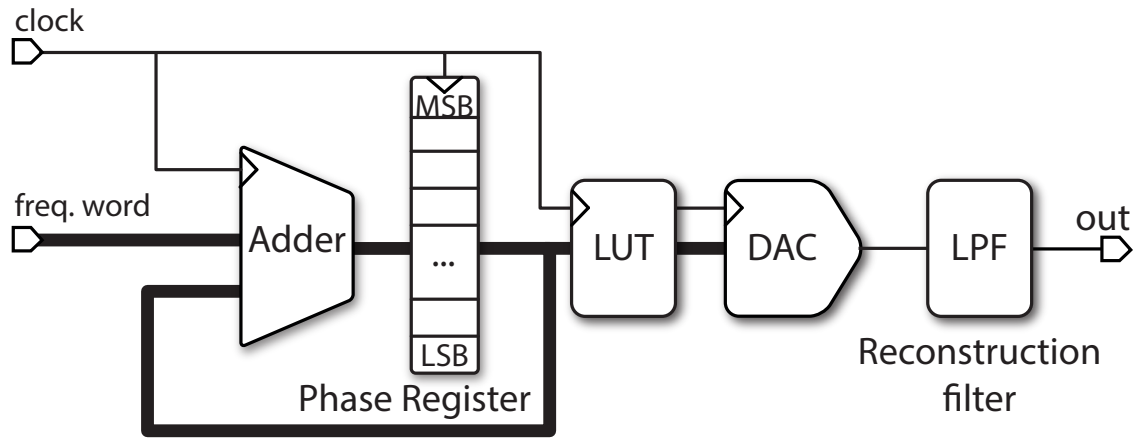

Figure 1.2: DFC producing a sine-wave. [CP:3]

applications allows it, where power savings are possible (Dynamical voltage and frequency scaling, DVFS) [10]. Moreover, radio communication requires very clean clocks with low spurs, to avoid mixing with unwanted channels. This is tied to the quality of the on-chip clock generator.

On-chip frequency generation is traditionally implemented with PhaseLocked Loops (PLLs) [11]. They can offer very high output frequencies with clean spectrum (low jitter) but have a limited output frequency range, limited by their Voltage-Controlled Oscillator (VCO) tuning range. Moreover, PLLs need time to settle after channel switching, e.g. because their loop bandwidth is limited due to stability requirements (locking time is tipically inversely proportional to the loop-bandwidth) [12]. The frequency resolution of integer-N PLLs is the reference frequency. This can be too coarse for many applications. Fractional-N PLLs overcome this limitation, but at the expense of the output spectrum quality.

A different approach for frequency generation is based on Direct Digital Synthesis. Direct Digital Synthesizers (DDSs), also called Digital-toFrequency Converters (DFCs) [7], have been known for decades [1, 2] but they are making a comeback $[13,14]$ due to the increasing integration density of the latest technological nodes, improvement in speed and especially power efficiency. A conventional DFC architecture exploiting a D/A converter that produces a sine-wave, shown in Fig. 1.2, usually reaches lower frequencies than a PLL, because, having so much digital content, it is competitive in terms of power consumption for frequencies below $1 \mathrm{GHz}$. However, a DFC can cover a much wider frequency range [15], essentially from arbitrarily low frequency to the maximum frequency. The digital nature of a DFC allows for more design automation, leading to short specs-to-product time and easy technology migration. Frequency resolution is low and the linear control on the synthesized frequency is convenient for modulation purposes. Most importantly, a DFC is a flexible clock source such that many frequencies can be generated from it and channel switching can be accomplished immediately. For these features, DFCs are an appealing approach for today systems' requirements in IC 


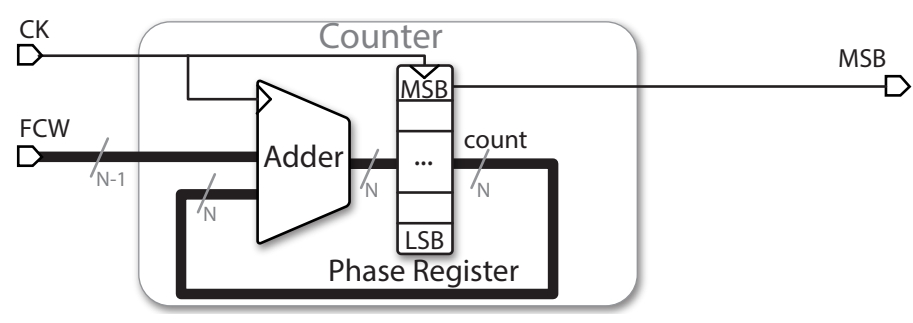

Figure 1.3: Scheme of a Pulse-Output DFC.

design. They allow area efficiency by significantly reducing the number of on-chip PLLs, because multiple DFC blocks can be attached to only one reference PLL, thus generating different frequencies all derived from and related to it. Also, pulling issues between PLLs or cross-talk issues between clock domains are avoided, as all timing is derived from a central reference clock.

However, DFCs have also some drawbacks. Conventional DFCs producing a clean sine-wave from digital sampled values via D/A conversion require a reconstruction filter. This results in issues like image frequencies that need to be suppressed, requiring bulky low-pass filters with an adequate stop-band rejection [16]. Sine-waves are not always needed as clock signal. Many circuits, like digital circuits, hard-switched mixers, samplers, and data converters can be and preferrably are driven by square-waves. In these cases, instead of producing a sine-wave first and then a square-wave (by passing the sinusoidal waveform through a hard-limiter), it is more efficient to exploit the digital core of the DFC to directly produce an output square-wave of the desired frequency. However, as will be explained in next section section 1.1, such output square-waves are affected by high deterministic jitter that appears in the output spectrum as a set of extra sub-harmonic tones (spurs). Therefore, these square-waves need compensation to realize a low spur output. This thesis focuses on the design and characterization of digital frequency synthesis for applications that require square-wave clocks, with a compensation mechanism to achieve low deterministic jitter.

\subsection{Deterministic jitter compensation}

In this section, we will briefly review the state-of-the-art compensation techniques applied to all-digital DFCs, for spur correction. These techniques have been extensively discussed in [7], while here we will focus on the main concepts.

All-digital DFCs are also named Pulse-Output DFCs. They differ from conventional DFCs in that they do not reconstruct sine-waves, but instead they have a fully-digital core consisting on just a phase accumulator, as shown in Fig. 4.2 (chapter 4), reproposed here in Fig. 1.3 for the reader's 


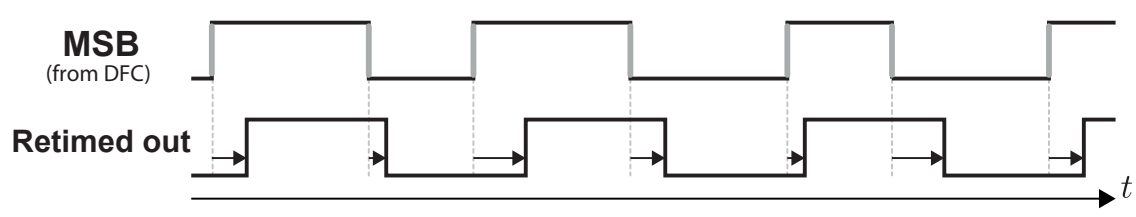

Figure 1.4: Retiming techniques.

convenience. The phase accumulator, triggered by the input clock, sums the input word FCW to the current value of the phase register. The most significant bit (MSB) of the sum is taken as the system output. The input word specifies the output frequency, that is why it is named Frequency Control Word (FCW)

Pulse-Output DFCs are based on the observation that the MSB of the phase accumulator contains all of the desired frequency information, so it has on average the target output frequency. Being the instantaneous frequency of the output not constant, the waveform is affected by deterministic jitter, which shows up in the spectrum as spurs.

Different techniques for spur correction has been proposed in literature and they can be combined with the Pulse-Output DFC of Fig. 1.3. These techniques can be classified in dithering, PLL and retiming techniques [7]. Digital random-dithering is a purely digital approach for spurs suppression. It is achieved by adding a random number sequence with zero mean to FCW (frequency dithering) [4], or by perturbing the output of the accumulator (phase dithering) [17]. Random dithering breaks the unwanted periodic patterns in the output sequences and spread the power of the corresponding spurs over a frequency continuum, thus converting the spurs' power into noise floor.

PLL techniques use a PLL as a frequency-translated band-pass filter, by locking it to the dominant tone of the Pulse-Output DFC [18]. Extra error canceling paths can be added $[19,20]$ to partially compensate the non-zero output of the PLL's phase-frequency detector.

Retiming techniques (Fig. 1.4) are based on delaying the output edges of the DFC to obtain square-waves with constant instantaneous frequency. The delay can be added in different ways. One method consist of connecting the phase register to a Digital-to-Analog Converter (DAC) and then send the resulting voltage level to a lumped transmission line, where varicaps affect delay when changing a control DC-voltage [19], as shown in Fig. 1.5. However, it is difficult to accurately control the desired delay values starting from the DAC voltage levels, due to Process-VoltageTemperature (PVT) variations.

Another way of adding delay is to employ the charging of a capacitor, as in the circuit in Fig. 1.6. The delay is controlled by acting on analog parameter, shown in the gray clouds in Fig. 1.6. The edge (rising or falling) of the MSB signal, coming from the DFC, starts the capacitor charging, thus producing a voltage ramp across the capacitor. The desirable delay 


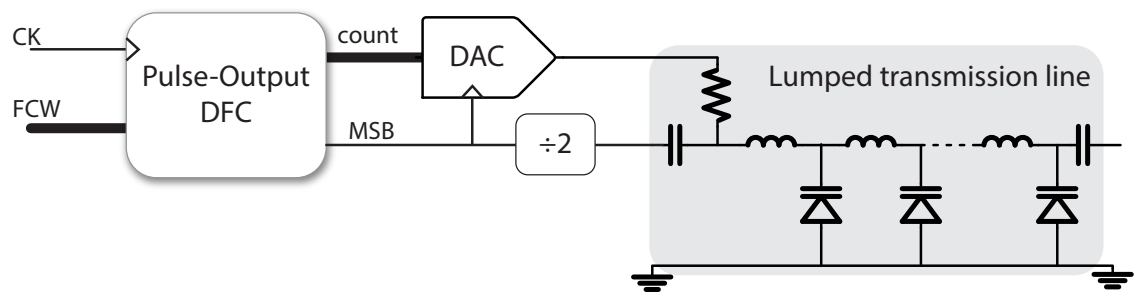

Figure 1.5: Pulse retiming using a lumped transmission line. Adapted from $[7,19]$

is obtained either adjusting the threshold voltage that the ramp should cross to produce a output edge [7], or adjusting the ramp slope by tuning the charging current [21-25]. The drawback for both of these methods is the poor linearity: implementing variable thresholds that change linearly with the desired delay is difficult, while variable slopes at a fixed threshold crossing affect the comparator's response, giving non linear switching instants [CP:2] .

A further way of delay generation is by using a delay line. It can be controlled either analogically, i.e. a chain of current-starved inverters [26], or digitally, i.e. with a tapped series of fixed delay elements (usually inverters) [27]. The first approach suffers more by PVT variations. The second one introduces a quantization of the delay values, limited by one gate delay, and suffers of delay variation over PVT, as well as accumulation of random jitter through the inverter chain.

The issues of the digital delay lines can be minimized by employing a Delay-Locked Loop (DLL) (Fig. 1.7). The clock at the output of the delay line is locked to the reference clock, thus reducing delay variations over PVT. Moreover the periodic realignment with the reference clock has a filtering action on the $1 / \mathrm{f}$ noise. The DLL can be used alone as in [28] or combined with other free running delay lines [29].

The same correction techniques recalled in this section can be also applied to Digital-to-Period Converters (DPCs) [30, 31], that are all-digital systems controlling the time-averaged output period. The key differences between all-digital DFCs and DPCs on system level will be discussed in section 4.2 .

\subsection{Research Goals}

The main objective of this $\mathrm{PhD}$ work is to investigate the usage of digitally programmable delays and their limitations, in the context of frequency synthesis. In particular, we will propose a Pulse-Output DFC based on a Digital-to-Time Converter (DTC) for deterministic jitter correction, as an alternative to other compensation approaches mentioned in section 1.1. This goal implies firstly a research focus on the DTC itself. 


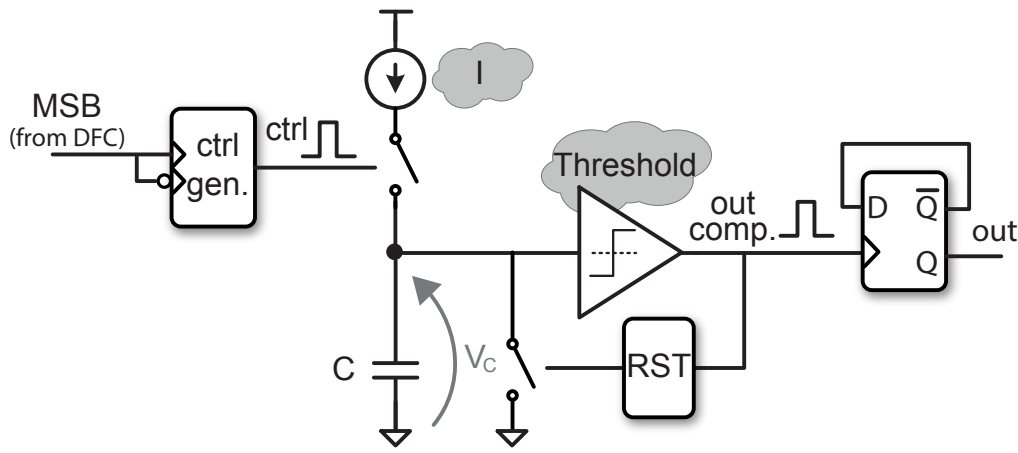

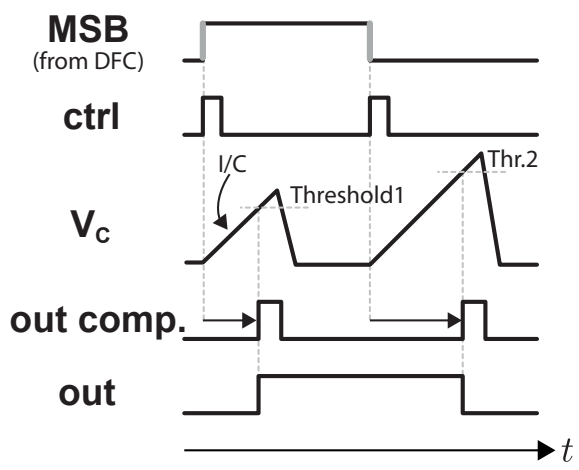

(a)

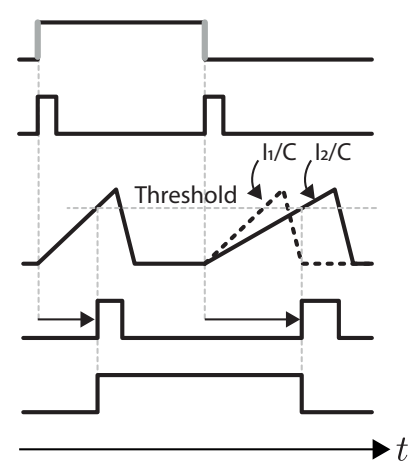

(b)

Figure 1.6: Retiming by capacitor charging. (a) controlling the threshold voltage; (b) adjusting the ramp slope.

DTCs are devices adding a digitally controllable delay to an input clock. Their implementation and measurement involve the capability to measure delays as small as hundreds of femto-seconds with tens of femto-seconds accuracy. Such a task is not obvious, since measuring delays below one pico-second is problematic even when using the best available high-speed oscilloscopes. Therefore, one of the first goals of this work is to set up a method that allows to measure reliably the DTC delay steps.

A second goal is the design of a linear DTC operating in the $\mathrm{GHz}$ range. We will first compare different DTC implementations already proposed in literature and, based in the insights developed, conceive a structure suited for the target frequency.

Furthermore, the proposed DFC with DTC correction will be modeled and analyzed from the perspective of its deterministic jitter, with the goal of setting the system specifications in terms of cleanness of the output spectrum and to study the limitations of the DTC and the effect of its impairments on the system output.

The goal of our research is to develop a fully integrated Pulse-Output DFC producing square-waves, with as low spurious tones possible in the entire range of the system output frequencies, and with a reasonable total 


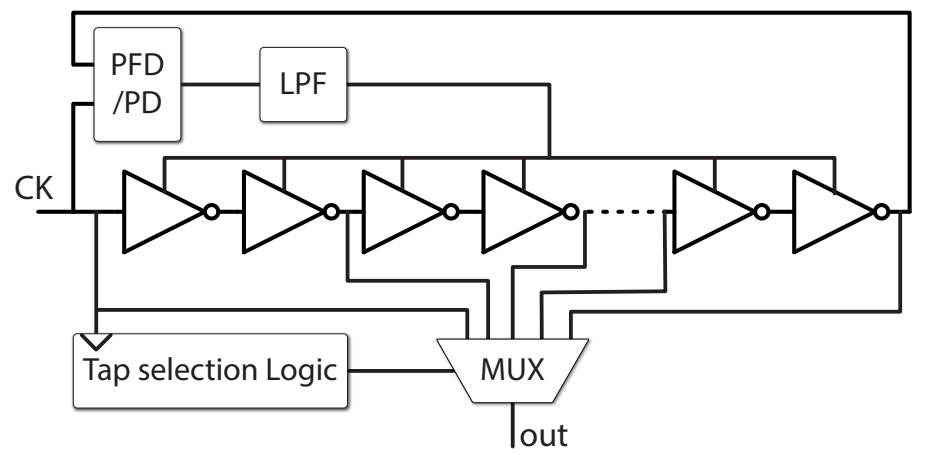

Figure 1.7: Pulse retiming using a DLL.

power consumption, aligned to the previous state-of-the-art.

\subsection{Thesis Outline}

This PhD thesis is organized as follows. Chapter 2 focuses on the stateof-the-art DTCs. Different delay mechanisms are shown and the existing DTC architectures are compared in terms of operating frequency, power consumption and linearity. The constant-slope principle will be discussed in this chapter, as it has been proven [CP:2] to increase the device linearity. We will call attention to the main DTC limitations for high-speed applications.

Chapter 3 describes the proposed method for accurate measurements of sub-ps delays. The method applies a digital phase modulation to the DTC and measures the correspondent delay indirectly, starting from the acquisition of the output with a spectrum analyzer, and then converting the measured frequency tones to the time domain.

Chapter 4 elaborates the behavioral model of the DFC with the DTC correction scheme. The design aspects of a DTC suitable for the target application and operating frequencies will be discussed and a set of key DTC parameters affecting the output spectrum will be identified. Moreover, we will provide a deep analysis of the output spurious tones and their behavior with the input digital word of the synthesizer.

Chapter 5 deals with the design of the proposed DFC prototype, based on the architecture and system considerations discussed in chapter 4 . The design is optimized for a wide range of output frequencies, from a few hundreds of $\mathrm{kHz}$ till around $800 \mathrm{MHz}$. The DTC operates at both rising ad falling edges of the output square-wave. Chapter 5 also shows measurements of the DTC linearity and output spectra, as well as a comparison with the analytical model developed in chapter 4.

Finally, the conclusions drawn from this thesis are summarized in chapter 6, along with a list of original contributions, and recommendations for future work. 
The appendices in this thesis provide proofs of some equations used in the analysis in chapter 4, as well as the Verilog code for the digital design flow (chapter 5) of the front-end in the DFC prototype. 


\section{State-of-the-art DTCs}

A Digital-to-Time Converter (DTC) delays an input clock according to a digital control word. It is suitable for several applications, each one requiring different operating frequencies of the DTC, from a few $\mathrm{MHz}$ to GHz. For example, fractional-N Phase-Locked Loops (PLLs) employ a DTC working at a few tens of $\mathrm{MHz}$, placed in the reference or the divider path, to relax the Time-to-Digital Converter (TDC) requirements [32-37]. Radar applications employ DTCs at around $10 \mathrm{MHz}$ to delay the sampling clock in the transmitter [38-40], or to span the timing-range of interest in the receiver [41]. In direct digital frequency synthesis, DTCs operating beyond $1 \mathrm{GHz}$ have recently started to be employed for spur mitigation in Digital-to-Period Converters (DPCs) and Digital-to-Frequency Converters (DFCs) [28, 42, 43].

Considering the retiming techniques mentioned in section 1.1, those controlled digitally can be regarded as implementations of DTCs. A broad definition of a DTC would include also tapped delay lines and DelayLocked Loops (DLLs) [44-46]. However in this thesis, we will strictly limit the term DTC to a "single shot" device that produces one output edge in response to an input edge. In fact, while tapped delay lines and DLLs produce multiple outputs, i.e. a lot of available edges to choose from, this also means that all edges except for one remain unused. A DTC, instead, produces only the edge needed, hence it is fundamentally more power efficient. The DTC delay steps can be designed to be much smaller than a CMOS gate delay, which is often the basis for DLLs. Furthermore, the DTC delay linearity is not limited by mismatches of different components associated with the creation of multiple delay taps in DLLs.

Notice that a DLL operates closed-loop and is a phase interpolator. It requires two edges to be present, between which it can place a new edge. Instead the DTC acts open loop. It often requires only one input edge to trigger the start of the delay and the delay is not constrained by a 


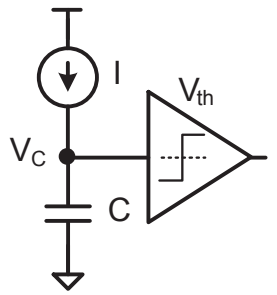

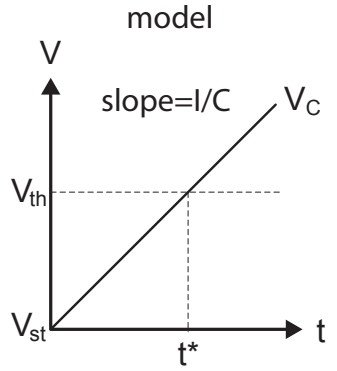

(a)

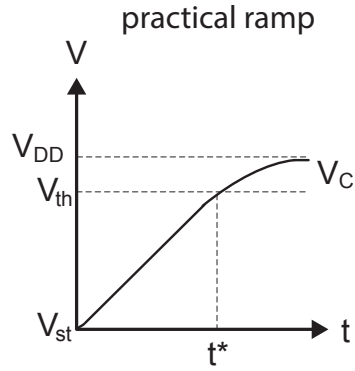

(b)

Figure 2.1: Voltage ramp for delay generation in a DTC: (a) ideal model; (b) a practical case. (Source: [CP:2] )

second edge. However some examples of open-loop DTCs acting as phase interpolators have also been proposed and will be discussed in this chapter, as well as other recent developments of high-resolution DTCs. The design efforts in literature are mainly focused on the delay generation part and most of the recent implementations exploit the constant-slope principle, discussed in next section.

\subsection{Constant-slope principle}

Linearity is an important metric in a DTC since it has a direct impact on performance in the systems in which the DTC is employed. For example, DTC non-linearity causes spurs in DTC-based frequency synthesizers [44, 47]. In this context, the Integral Non-Linearity (INL) is a key specification for a DTC, where the delay produced by the converter varies widely over the full-scale, thus making the INL more important as a metric than the Differential Nonlinearity (DNL).

Design efforts to increase the DTC linearity can be directed either to ana$\log$ design techniques [CP:2], or to the development of digital algorithms for pre-distortion and multipoint calibration [48]. The latter techniques can be convenient if they allow to reduce the DTC complexity substantially. However calibration depends on the architecture of the system in which it is applied and analog techniques are still required to avoid changes in INL that would not be compensated. Therefore, a intrinsically linear relation between the DTC delay and its input control word is favored, as calibration of only two points is sufficient. In view of this, the constant-slope principle has been employed for delay generation, for its linear behavior.

A DTC often exploits a voltage ramp generated by a current source charging a capacitor, followed by a Zero Crossing Detector (ZCD) that switches when the ramp reaches its threshold (Fig. 2.1). A CMOS inverter can also be seen as a voltage ramp generator, since the active MOSFET in saturation is practically a current source charging the output capacitance. The digital control of the delay is usually achieved by programming a 


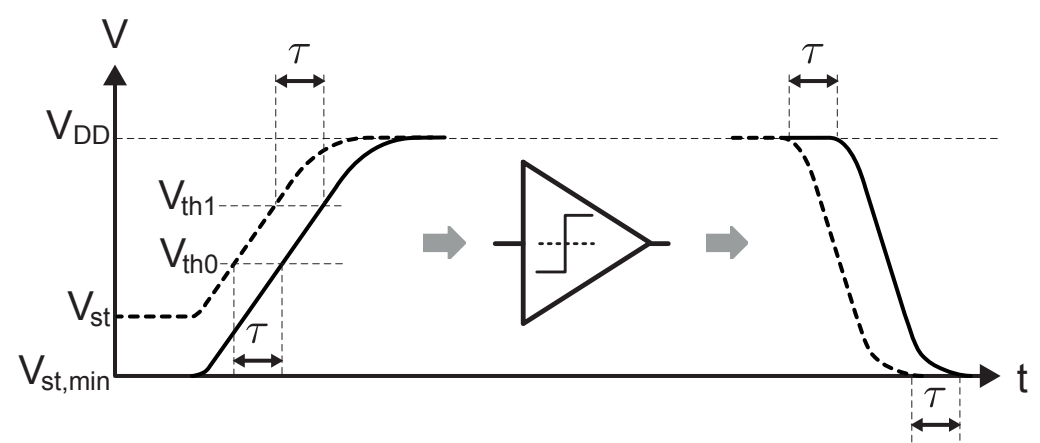

Figure 2.2: Delay mechanism for the constant-slope method: the start voltage linearly affects delay, while the ZCD response is identical for all the ramps and because they have the same shape in the ZCD window. (Source: [CP:2] )

bank of capacitors $[49,50]$ or by switching the current sources [51-53]. This approach has been called variable-slope method in [CP:2], because the delay control variable is the ramp slope or slew-rate (I/C in Fig. 2.1a). With this technique, a $550 \mathrm{fs}-$ resolution DTC with $1.8 \mathrm{LSB}$ INL and working at $40 \mathrm{MHz}$ has been implemented in [50].

In contrast, the constant-slope principle uses the starting voltage of the ramp ( $V_{s t}$ in Fig. 2.2) as delay control variable, while keeping its slope fixed. The increased linearity of the constant-slope delay mechanism over the variable-slope one comes from the behavior of a practical ZCD. For a input ramp signal, the ZCD propagation delay varies in a non-linear way with the input ramp time, due to the detector bandwidth limit. This can be explained by noticing that the bandwidth limit is equivalent to an $\mathrm{RC}$ network added at the detector output, and that the propagation delay of an RC network of any order contains nonlinear functions of the input ramp time [54,55]. The constant-slope method keeps the input ramp time constant with the input code, thus the ZCD response time is a constant time offset that does not affect the linearity. Moreover, if the ZCD passes through different operating modes during its input and output transitions, the fixed input ramp time provided by the constant-slope method makes the operating modes contribute always in the same way to the output transition time, thus avoiding another source of INL [CP:2] .

This concept is shown in Fig. 2.2. Two rising ramps at the input of the ZCD have the same shape up to the decision moment, but different starting voltages. The ZCD senses the delay $\tau$ between the two input waveforms and produces two corresponding falling edges at the output. Actually it is more appropriate to talk about a ZCD threshold window, rather than a level, so Fig. 2.2 shows the two window edges $V_{\text {tho }}$ and $V_{\text {th } 1}$. The ZCD does not switch when its input is outside these edges, so only the part of the ramps within the threshold window affects the output. Two waveforms having the same shape inside the ZCD window evoke the same response 


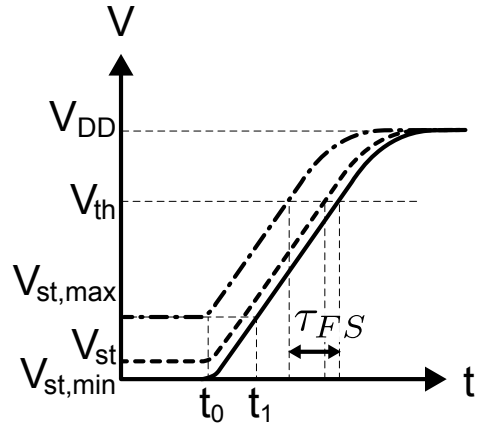

(a)

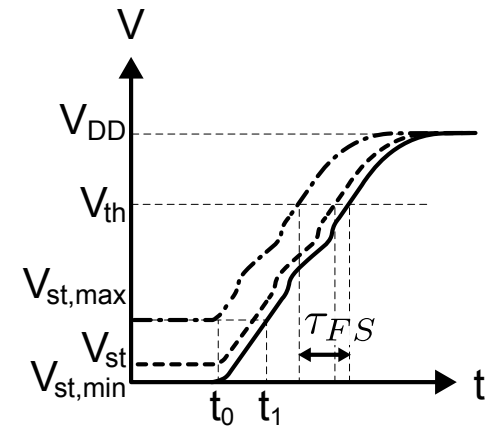

(b)

Figure 2.3: Constant-slope principle: (a) simplified scheme, with fixed slope during the entire ramp; (b) sufficient condition, with fixed slope between the instants $t_{0}-t_{1}$ and constant shape elsewhere, until saturation to supply; $\tau_{\mathrm{FS}}$ is the DTC full-scale delay as it corresponds to the maximum and minimum starting voltages.

at the output. Hence their propagation delays through the ZCD are equal, and both the output edges also have a constant shape, no matter what the bandwidth is. Thus, ideally the delay $\tau$ between the outputs is the same as the one between the inputs. Moreover, as the ZCD outputs are constant-shape, the following stages (e.g., buffers) will also not introduce INL errors.

To guarantee a linear delay control, it is sufficient that the constantslope voltage occurs in the part below the maximum starting voltage $V_{s t, \max }$ (see Fig. 2.3), while for the part above it suffices to have a constantshape over different input codes. This property allows for a more general interpretation of the name constant-slope, i.e. the same (local) slope at equal voltage. This is shown in Fig. 2.3b: during the time interval $t_{0}-t_{1}$, a linear mapping occurs between starting voltages and times, thus the ramps in this interval should have a fixed slope. A constant local slope at all the voltages in the range $V_{s t \text {, max }}-V_{t h}$ will not affect the linearity of the crossing instants.

The constant-slope principle presents also limitations compared to the variable-slope approach. A very important issue is the non-zero start-up time. At the start of the DTC delay generation, the starting voltage should be set, as shown in Fig. 2.4. Moreover, even for the maximum starting voltage $V_{\mathrm{st} \text {, max }}$, time is required for the input waveform to reach the ZCD threshold window (dead-time). Fig. 2.4 illustrates the time slots required for a constant-slope DTC. The effective threshold $V_{t h}$ of the ZCD is in the middle of the threshold window. We will name $\tau_{\text {oft }}$ the total time offset (start voltage setting + dead time) before the DTC programmable delay range. Since the shape of the voltage waveforms should be kept the same, it is not possible to reduce $\tau_{\text {oft }}$ by increasing the input slopes for lower codes, 


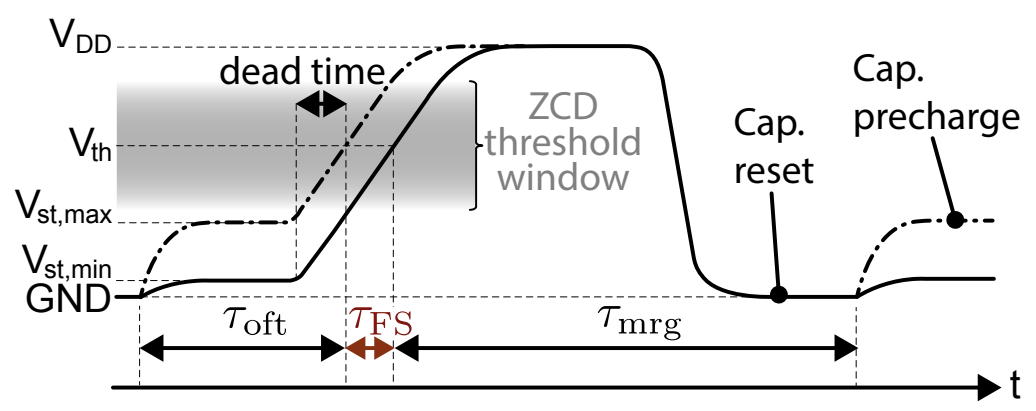

Figure 2.4: Time slots in a constant-slope DTC.

as done in variable-slope DTCs. The usable range of starting voltages is limited, since the maximum starting level $V_{s t, \max }$ should be lower than the ZCD threshold window for a correct detection. Therefore, achieving a larger delay requires a lower slope (i.e., slower ramp), thus increasing the start-up time and setting a delay-jitter trade-off. Moreover after producing the delay, some time margin $\tau_{\mathrm{mrg}}$ in Fig. 2.4 is needed to reset the capacitor voltage, effectively wiping out history, which is important to avoid history dependent outcomes (i.e. nonlinearity). Hence, as one constant-slope DTC is not available full-time, it is

$$
\tau_{\mathrm{FS}}<1 / \mathrm{f}
$$

where $\tau_{F S}$ is the DTC full-scale delay and $f$ is the DTC operating frequency. Note that a time offset and time margin are also needed for a variable-slope DTC but for a constant-slope DTC the time offset cannot be reduced by changing the input slopes for lower codes. This is the penalty for the higher linearity achieved with the constant-slope approach.

This thesis targets a DTC architecture based on the constant-slope principle which can operate at much higher frequencies than demonstrated before. We aim for at 10x faster operation than [CP:2] , i.e. > $1 \mathrm{GHz}$ instead of $100 \mathrm{MHz}$. Moreover we will try to improve the supply sensitivity and cross-talk by adopting a differential circuit approach. Innovations in the system architecture, will be addressed in section 4.3.3, while the differential circuit implementation will be examined in section 5.3.

\subsection{Architectures}

The circuit schematic of the constant-slope DTC proposed in [CP:2] is shown in Fig. 2.5. The DTC delays only the rising edges of the input clock. For this reason, the input buffer contains big NMOS transistors for low noise and its PMOS, with much smaller channel width, is driven appropriately to minimize the short circuit currents. The circuit sweeps through three states, reset, precharge and charge state, controlled by the signals $V_{\text {res }}, V_{\text {prech }}$ and $C L K_{i n}$. The capacitor $C_{0}$ is first reset to ground, then 


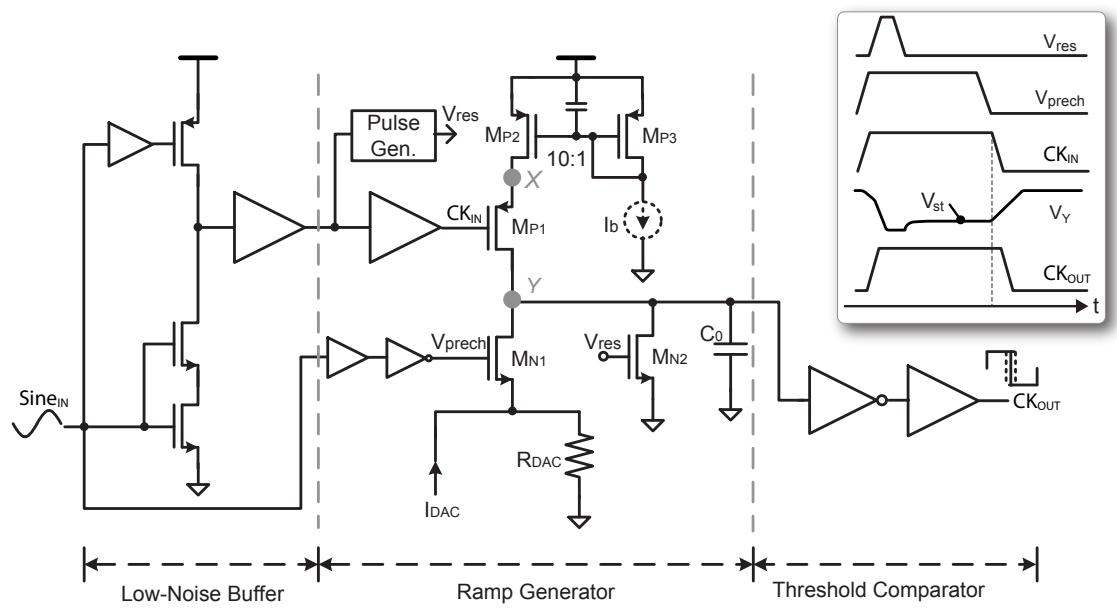

Figure 2.5: Architecture of a constant slope DTC for $\mathrm{MHz}$ operating frequency. (Source: [CP:2] )

charged to the starting voltage $V_{s t}$ (precharge state) and finally charged with a constant current to develop a voltage ramp. The current source is made of PMOS transistors, with transistor $M_{P 1}$ acting both as cascode and switching element to start the charging of the capacitor $\mathrm{C}_{0}$. The starting voltage is determined by a 10-bit current-steering Digital-to-Analog Converter (DAC). The comparator is implemented with an inverter followed by a buffer. The inverter has a threshold voltage at around half- $V_{D D}$ $(600 \mathrm{mV})$, that is much larger than $V_{s t \text {, max }}$, while the final buffer is made of two scaled-up inverters which steepen the output edges. Fig. 2.6 shows the measured INL with 189 ps full-scale and $55 \mathrm{MHz}$ operating frequency. The maximum INL is $328 \mathrm{fs}$, corresponding to $0.17 \%$ of the full-scale and to an effective resolution of 9.2 bits. The power consumption is $1.8 \mathrm{~mW}$.

Another DTC architecture using the constant-slope principle is in [56], targeted to minimize the power consumption. It acts on the rising edges of the output. Likewise the DTC in [CP:2], the delay control variable is the starting voltage of a ramp. Other similarities include the operating frequency in the $\mathrm{MHz}$ range ([56] working at $40 \mathrm{MHz}$ ) and the action on only one type of input clock edges (rising edges only). To improve the power efficiency, the architecture is simplified to only a input buffer, a couple of inverters, and a DAC. The current source that acts as ramp generator is the PMOS of the first inverter, without a cascode stage. The resulting stronger dependence of the current on the starting voltage limits the full-scale range to only 5-bits [56], to guarantee an INL below 1 LSB. The DAC chosen to set the starting voltage of the ramp is capacitive. In this way, its consumes significantly lower power compared to other DAC architectures, as it requires only dynamic power to charge the capacitors and the inverter cell becomes the main power-consuming block in the ramp 


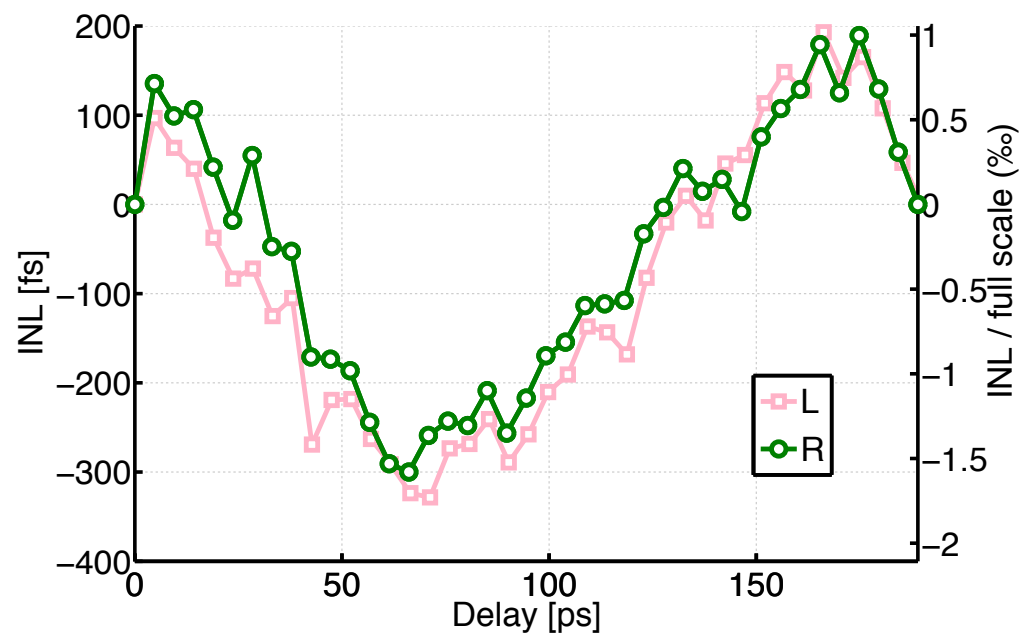

Figure 2.6: Measured INL of the constant-slope DTC in Fig. 2.5. (Source: [CP:2] )

generator. The DTC in [56] is implemented in TSMC 28-nm LP CMOS Its power consumption is $15 \mathrm{uW}$, with a resolution of $103 \mathrm{fs}, 3.3 \mathrm{ps}$ full-scale, and a INL of 0.73 LSB.

The DTC implementation in [57] is targeted to GHz frequencies. It is an open loop phase interpolator, since it needs two input edges to set the starting level of the voltage ramp. Its architecture, shown in Fig. 2.8a, is based on dividers to provide the coarse delay, thus ensuring low jitter and high power efficiency. The DTC has 11-bit resolution, and it is segmented into a 3-bit Multi-Modulus Divider (MMD) for ultra-coarse tuning, 1-bit coarse tuning realized with a multiplexer + delay element, and a 7-bit phase interpolator (block DCEI) for finer time resolution.

The $8 \mathrm{GHz}$ VCO signal, provided by a PLL, is divided inside the MMD block, with nominal division ratio of 4 , into the $2 \mathrm{GHz}$ signals $M M D_{\text {out, } 1}$

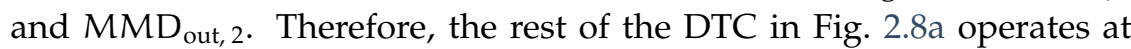
$2 \mathrm{GHz}$. The bits $\mathrm{DW} W_{10: 8}$ determine the relative phase between $M M D_{\text {out, } 1}$ and $M M D_{\text {out, } 2}$ and which signal leads or lags the other one. Thus, overall the first MMD block provides a first 3-bit ultra-coarse delay over the $2 \mathrm{GHz} 2 \pi$ full-scale delay range.

The DCEI block interpolates on rising and falling edges of the $2 \mathrm{GHz}$ clock, but the delay word DW is latched at every falling edge of the DTC output (not shown in Fig. 2.8). This does not allow to arbitrarily control the output duty-cycle, because the output rising and falling edges cannot be changed independently, but it can be enough to keep the duty-cycle at $50 \%$.

After the ultra-coarse block MMD and the coarse stage MUX+DEL, the period $1 / 2 \mathrm{GHz}=500 \mathrm{ps}$ is divided into 16 segments and two interpolating 


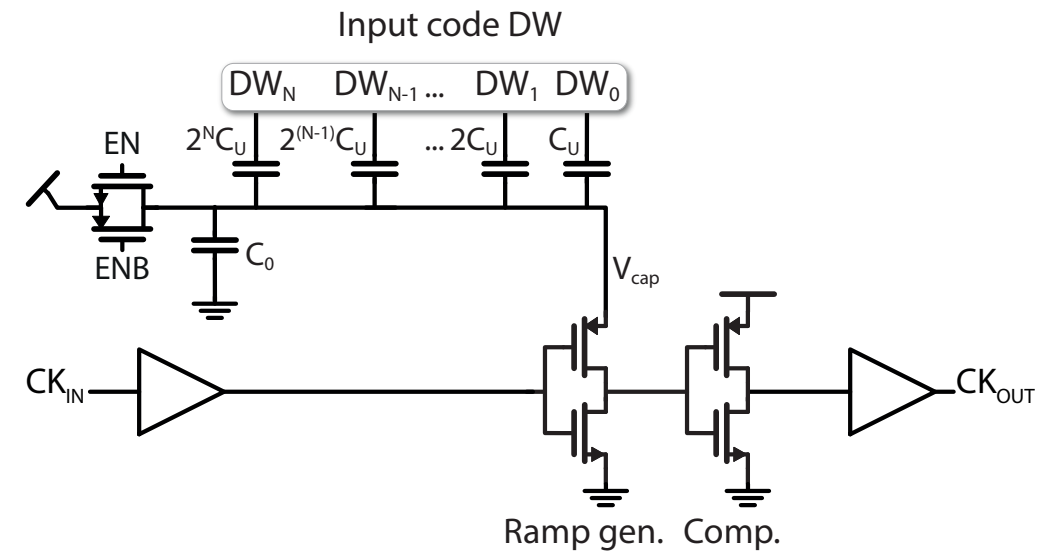

Figure 2.7: Low-power constant-slope DTC. (Source: [56] )

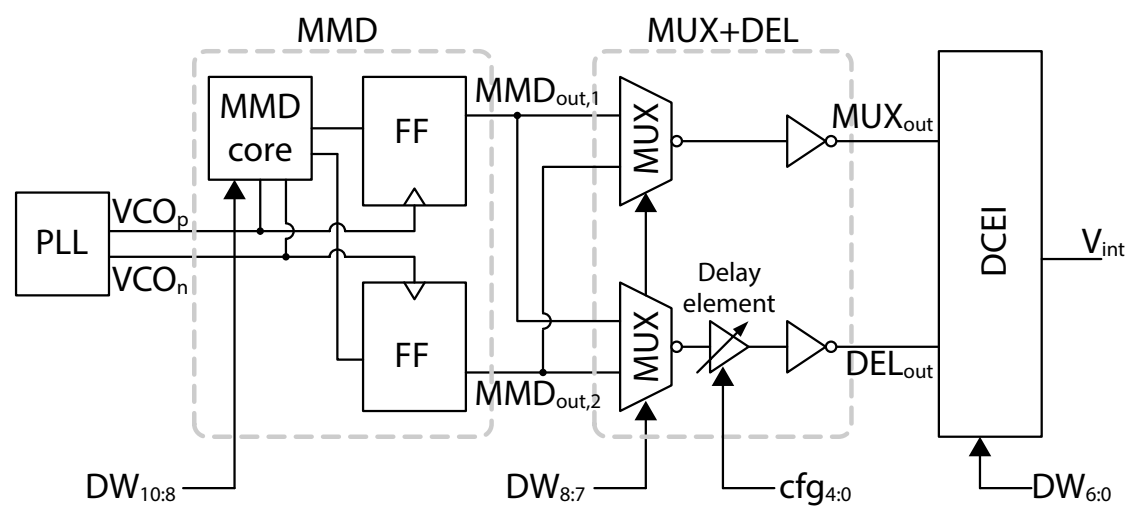

(a) Architecture overview.

Digitally Controlled Edge
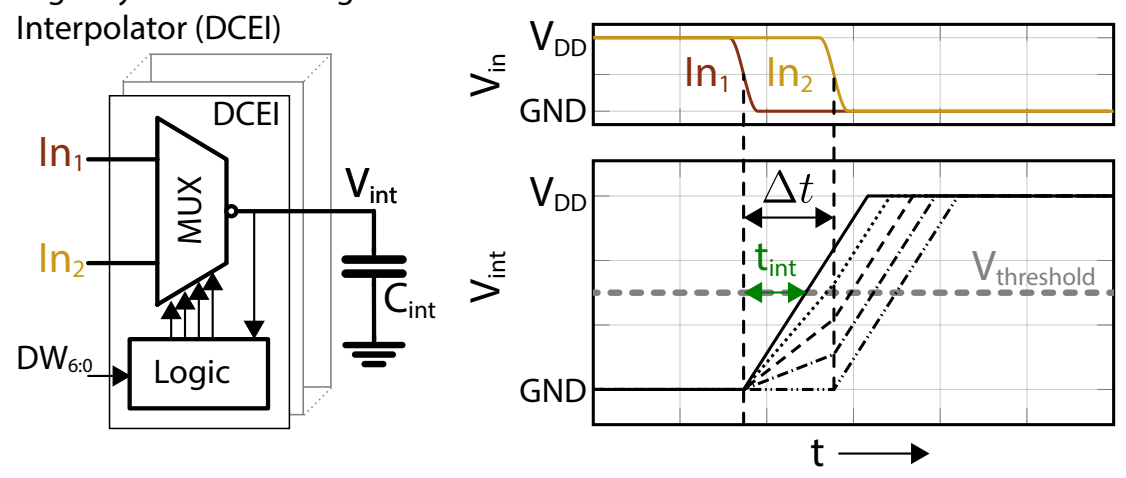

(b) Interpolation cell.

Figure 2.8: Implementation of a DTC targeted at GHz operating frequency. (Source: [57] ) 


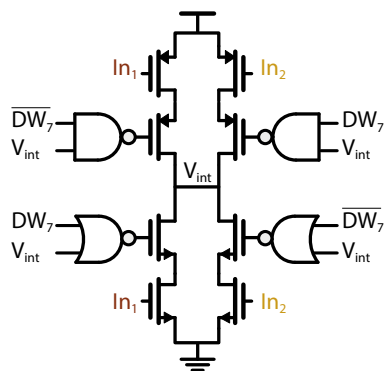

(a)

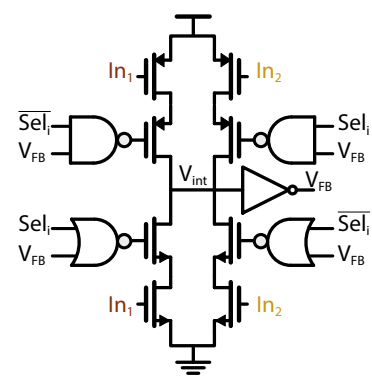

(b)

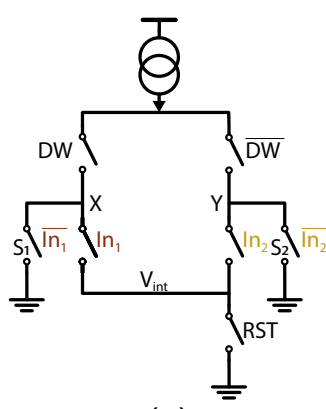

(c)

Figure 2.9: (a) Retention and (b) Interpolation cells in the DTC of Fig. 2.8 [57] (c) Modified interpolation cell in [58].

signals, $\mathrm{In}_{1}$ and $\mathrm{In}_{2}$ are generated (shown in Fig. 2.8b). These signals will interpolate the fine delay. $\mathrm{In}_{1}$ is always the leading signal and it can correspond to $M U X_{\text {out }}$ or DEL out depending on the delay word DW. $\mathrm{In}_{2}$ will be the other signal. Two consecutive edges of $\mathrm{In}_{1}$ and $\mathrm{In}_{2}$ are always delayed by $\Delta t=31.25 \mathrm{ps}(1 / 16$ of the period $500 \mathrm{ps})$. The fine delay is interpolated in the following way. The 128 DCEI blocks (7 bit) are all connected to the integration capacitor $\mathrm{C}_{\text {int }}$ (Fig. 2.8b). During the interpolation time $\Delta t$ (Fig. 2.8b), each DCEI block can provide a current in the capacitor $C_{\text {int }}$ or no current, depending on the fine-word $D W_{6: 0}$. Therefore, during $\Delta t$, a code-dependent slope is generated, because the total charging current in $\mathrm{C}_{\text {int }}$ depends on the number of DCEI blocks acting as current sources. The arrival of the $\mathrm{In}_{2}$ edge determines the end of the time interval interpolation time $\Delta \mathrm{t}$. This results in a code-dependent interpolated voltage at the end of $\Delta t$, as shown in Fig. 2.8b. After that, all the DCEI cells contribute to the charging of $C_{\text {int }}$ and the ramp always has the maximum slope.

The ZCD that follows $C_{\text {int }}$ (not shown in Fig. 2.8) is implemented with an inverter and has a nominal threshold voltage $V_{\text {threshold }}$. The time interval $t_{\text {int }}$ shown in Fig. $2.8 b$ is the time needed for the ramp voltage $V_{i n t}$ to grow from ground to $V_{\text {threshold }}$. Notice that the offset time $t_{\text {oft }}$ discussed in section 2.1 and in Fig. 2.4 for the constant-slope principle corresponds to $\mathrm{t}_{\text {oft }}=\max \left\{\mathrm{t}_{\text {int }}, \Delta \mathrm{t}\right\}$.

It should be noticed that if $t_{\text {int }}>\Delta t$, the DTC would operate in constant-slope, at the expense of a longer offset time. However, in [57], $\Delta t$ has been chosen a few pico-seconds longer than $t_{i n t}$, so the situation pictured in Fig. 2.8b applies. Hence, as can be seen from the threshold crossing in Fig. 2.8b, the crossing is variable-slope for lower delays (crossing inside $\Delta t$ ) and constant-slope for higher delays, when the crossing occurs after $\Delta t$. Consequently, the output is affected by a INL due to first order to the variable-slope portion of the threshold crossings, that has been modeled in [57]. In conclusion the trade-off is between speed and linearity.

The schematic of an interpolation cell, inside each DCEI block, consists 


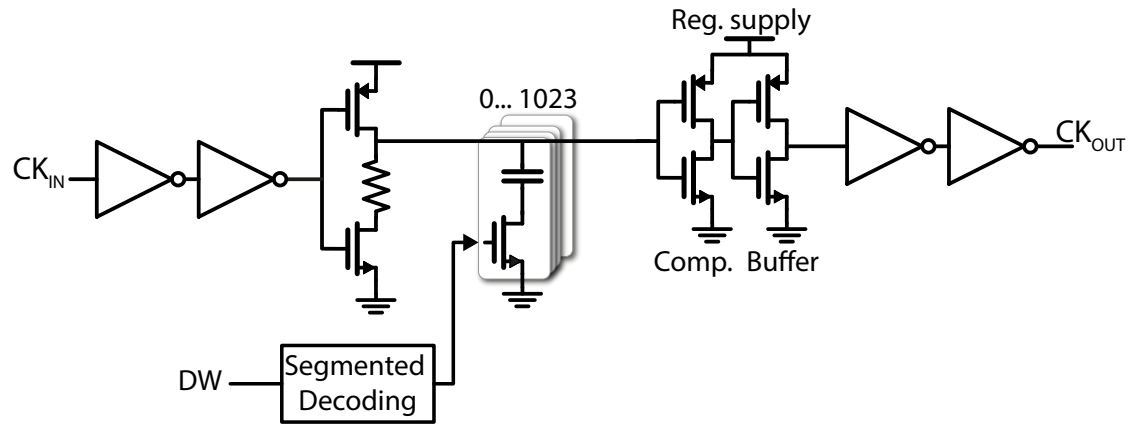

Figure 2.10: Variable-slope DTC for $\mathrm{MHz}$ operating frequency. (Source: [50])

of a CMOS multiplexer based on two tristate inverters and it is shown in Fig. 2.9b. A logical feedback involving a signal called $V_{F B}$ in Fig. 2.9b guarantees that only one branch per cell is active during the interpolation, thus preventing cross-bar currents between the power supply and ground, which are the main cause of non-linearity. The interpolation cells are arranged in a matrix. In between interpolations, there is no conducting path in the interpolation cell, leaving the output node $V_{i n t}$ floating. In this way, $V_{\text {int }}$ would be susceptible to noise, thus becoming a non-deterministic source for non-linearity. Therefore, retention cells, shown in Fig. 2.9a, are inserted in each column of the matrix, in order to hold the state of $V_{\text {int }}$. The DTC, implemented in standard 28-nm CMOS, has a resolution of $244 \mathrm{fs}$, a peak INL of $1.2 \mathrm{ps}$, corresponding to $4.92 \mathrm{LSB}$, and a power consumption of $19.8 \mathrm{~mW}$.

A variation of [57] is the DTC in [58], where the unit cell in Fig. 2.9b has been modified to the version in Fig. 2.9c. In this way, the charging current is not dependent on the CMOS switch resistance and therefore it does not depend on the parasitic resistance and on the voltage of the output node. The two switches $S_{1}$ and $S_{2}$ have been added to discharge the parasitic capacitors before a new interpolation phase starts. This avoids the DTC linearity to be sensitive to the code-dependent voltage of the parasitic capacitors at nodes $X$ and $Y$ in Fig. 2.9c. Moreover, the DTC operates at $t_{\text {int }}>\Delta t$, so it is a truly constant-slope device. It achieves, $2.5 \mathrm{GHz}$ operating frequency, $100 \mathrm{fs}$ resolution, a peak INL of 3.83 LSB and a power consumption of $7.1 \mathrm{~mW}$ available in simulations. Unfortunately no measurements are shown in [58].

The DTC in [50] (Fig. 2.10) is instead a standard application of the variable-slope principle, for $\mathrm{MHz}$ frequencies and with control on output falling edges. It is relevant because it contains several techniques focused to achieve low phase-noise and high-linearity. The delay element is an inverter, followed by a digitally-programmable capacitive load. The DTC 


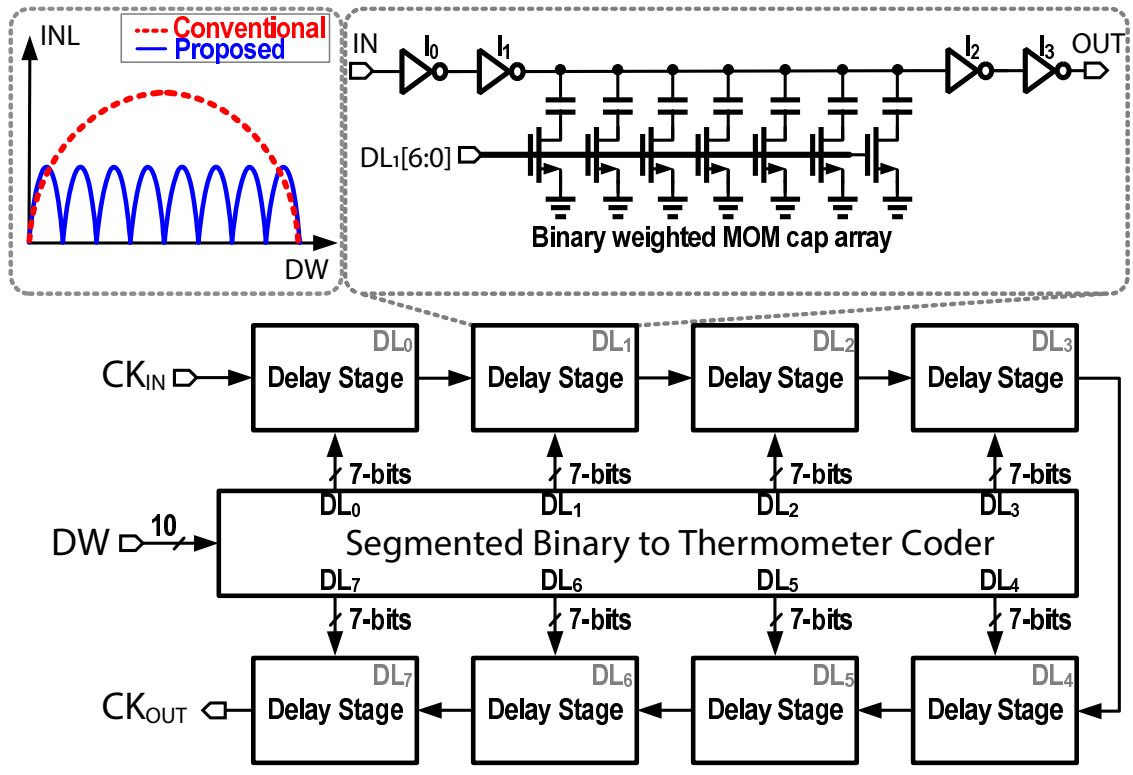

Figure 2.11: Multi-stage variable slope DTC. (Adapted from: [61])

is meant to be used inside a sub-sampling PLL [59, 60], where only falling edges (turning the sampling switch off) are relevant for the system operation. Therefore, only the DTC falling edges have been optimized for low noise. As the flicker noise is dominant in the delay element, a resistor has been placed above the nMOS of the inverter, as shown in Fig. 2.10. In this way, the capacitor discharging is determined only by passive components, capacitor and resistor, so the flicker noise source is removed. As for the INL, [50] addresses the impact of the supply ripple, that couples into the delay path. The ripple is reduced by making the inverters of the delay elements minimum length. This is possible since the nMOS inside the inverter does not control the output falling edge anymore. Moreover, the supply ripple is made colorless (i.e. independent from the input delay word) by adding a replica of the sensitive delay stage, in parallel to the main delay path, fed with a complementary code.

The work [61] proposes a $100 \mathrm{MHz} 10$-bit DTC, based on digitally controlled delay lines (Fig. 2.11), made out of CMOS inverters loaded by a capacitor array. Although the delay mechanism is variable-slope, a good linearity ( 5 LSB peak INL) is achieved by using different tricks. Firstly the segmentation, with 3 thermometer-coded MSBs (for coarse delay) and 7-bit binary delay lines (for fine delay), helps to reduce the fine full-scale. Since the linearity of capacitively-loaded delay lines is proportional to the stage delay [48], this trick decreases the dominant $2^{\text {nd }}$ order non-linearity, as shown in Fig. 2.11. Secondly, the problem of code-dependent current modulation due to finite supply impedance is addressed by adding a 

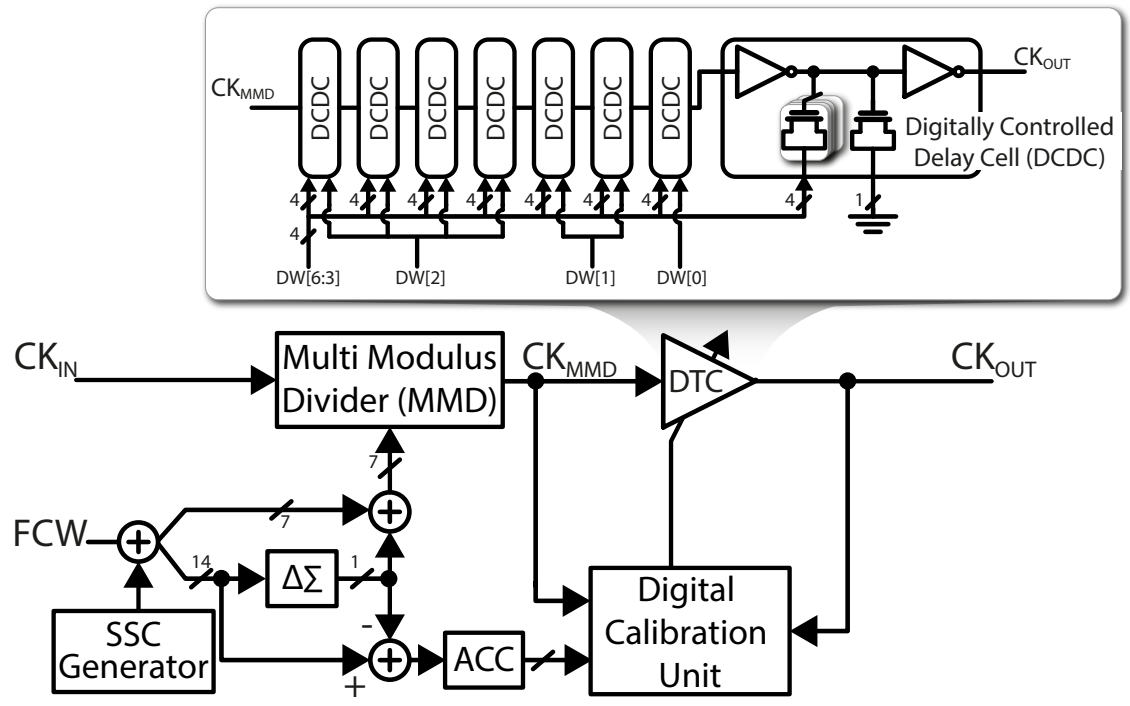

Figure 2.12: Divider-based frequency synthesizer with multi-stage variableslope DTC operating at GHz. (Adapted from: [28])

dummy delay line driven by a complementary input word, to make the required supply current code-independent. Thirdly, using switched capacitors (MOS switches combined with MOM capacitors) instead of MOS varactors improves the coupling-immunity of the capacitance array because control signals are routed instead of sensitive capacitor terminals. The input DTC code is reset to full-scale at the end of each conversion cycle, to connect all the MOM capacitor to ground, thus avoiding floating nodes which are other potential sources of non-linearity. Finally, inserting the additional inverters $\mathrm{I}_{0}$ and $\mathrm{I}_{3}$ allows for increasing the edges rates and to optimize the inner inverters, $\mathrm{I}_{1}$ and $\mathrm{I}_{2}$ for linearity.

A similar DTC in [61] has been used in the Direct Digital Synthesizer (DDS) in [28], based on $\Delta \Sigma$ fractional dividers (Fig. 2.12). The 7-bit DTC cancels the quantization noise of the clock CLK $_{M M D}$ coming from the multimodulus-divider. The DTC has a segmented architecture with $4 \mathrm{MSBs}$ (DW [6:3] in Fig. 2.12 and 3 LSBs (DW [2:0]). It consists of 8 delay cells made of a CMOS inverter loaded with a tunable 16-unit capacitor bank: the 4 MSBs of the delay word DW control 15 capacitors in all the delay cells, while each of the 3 LSBs control one unit capacitor in different delay cells. This segmentation scheme helps to maintain almost the same rise/fall time among all the stages, thus improving the DTC INL. The maximum current spike drawn from the supply is reduced by the choice of a segmented architecture. The DTC in [28] achieves around 1 LSB INL and operates at maximum $1 \mathrm{GHz}$. To perfectly cancel the quantization noise, the DTC gain has to be calibrated to match the input clock period, using a digital calibra- 
tion unit. In $[28,61]$, a DLL is used for the DTC background calibration, while the delay itself is generated open loop by the DTC.

\subsection{DTC benchmarking}

Similarly to Data Converters, DTCs are benchmarked in terms of resolution, power consumption, frequency range, full scale delay and linearity. Recent publications $[56,61]$ used a Figure-of-Merit (FoM) for DTCs, indicating the average required energy per conversion:

$$
\mathrm{FoM} \triangleq \frac{\mathrm{P}}{\mathrm{f}_{\mathrm{DTC}}} \frac{\mathrm{INL}}{\text { Delay Range }} \frac{1}{n_{\text {edges }}}
$$

Where $P$ is the power and $f_{\text {DTC }}$ the DTC frequency. All the DTCs mentioned in section 2.2 are compared in Table 5.3 in chapter 5, together with the performances of the proposed DTC designed for the prototype. The FoM of the most recent implementations is a few tens of $\mathrm{fJ} /$ conversion.

\subsection{Conclusions}

DTCs suitable to be used inside DFCs should preferrably have a high operating frequency, beyond $1 \mathrm{GHz}$, sub-picosecond linearity, with FoM of a few tens of $\mathrm{fJ} /$ conversion (section 2.3). All the best-performance DTCs are someway related to the constant-slope principle [CP:2], producing a constant-slope voltage ramp on a capacitor with digitally programmable initial voltage, and then detecting the threshold crossing of the voltage ramp (section 2.1). Even in the case of DTCs based on a variable-slope voltage, high linearity is obtained by using multiple stages producing slopes that vary at most by 1 LSB ([28] reported in section 2.2), so in a way the superior linearity of the constant-slope approach is also exploited there. Different DTC architectures have been discussed in this chapter, where the innovations over the years have been focused on improving the DTC speed and power efficiency. 



\section{CHAPTER}

\section{A Sensitive Method to Measure the INL of a \\ DTC}

A Digital-to-Time Converter (DTC) produces a time delay based on a digital code. Like for data converters, linearity is a key metric for a DTC and it can be characterized by its Integral Non-Linearity (INL). However, measuring the INL of a sub-ps resolution DTC is problematic even when using the best available high-speed oscilloscopes.

In this chapter, we propose a new method to measure the INL of a DTC, by applying digital phase modulation and measuring the output spectrum with a spectrum analyzer. The frequency selectivity of this method allows for improved measurement resolution down to a few fs and allows to measure a INL below $100 \mathrm{fs}$. The proposed method is verified by behavioral simulations and employed to measure the INL of a high-resolution DTC realized in $65 \mathrm{~nm}$ CMOS, with time-resolution of $25 \mathrm{fs}$ and standard deviation of $27 \mathrm{fs}$.

\subsection{Introduction}

Time or clock generation with high fidelity is at the heart of numerous electronic systems. The rapid development in Time-to-Digital Converters (TDCs) and Digital-to-Time Converters (DTCs) [62], that are increasingly used in Phase-Locked Loops (PLLs) [60, 63, 64], pushes the required time resolution to well below $1 \mathrm{ps}$. This chapter targets the measurement of such small timing steps. The principal instruments traditionally employed for such measurements are the network analyzer or the oscilloscope.

Network-analyzer-based measurement methods quantify the phase difference between two sinusoidal signals, generated by the same source and

This chapter has been previously published in [CP:2] 
passing through two different paths [65]. This phase difference is translated into a time difference, assuming accurate knowledge of the carrier frequency. Processing algorithms applied to the detected phase difference allow to achieve more than 10 ps time accuracy with these methods [66]. However, they are not suitable to measure time differences between nonsinusoidal digital signals.

Oscilloscope-based time measurements are applicable to digital signals. The achievable time measurement resolution depends amongst others on the bandwidth and the accuracy of the oscilloscope's sampling clock. The latest commercially available oscilloscopes can provide a sample clock jitter of $75 \mathrm{fs}$, with a delta-time measurement accuracy in the order of $500 \mathrm{fs}$ for rail-to-rail digital signals [67]. This is just enough to measure 1.25 ps resolution of the state-of-the-art TDC $[68,69]$, or $550 \mathrm{fs}$ resolution of the latest DTC [50], but it is insufficient to measure time delays in the order of 100 fs or below.

Aiming to overcome the oscilloscope's resolution and accuracy limits, in this chapter we propose a new method for time measurements that uses a spectrum analyzer as principal instrument. The proposed method is specially devised for a DTC and is based on digital phase modulation, while observing the output spectrum. A DTC produces a delayed version of its clock, controlled by a digital input code. It has gained renewed interest especially in the PLL research field $[60,63,64]$, because it can be used inside a PLL to relax the requirements of the TDC. Similarly to data converters, Integral Non-Linearity (INL) is an essential metric also for time converters (DTCs and TDCs).

The traditional way to measure the INL of a DTC is oscilloscope-based: the oscilloscope detects the time difference between the threshold-crossing points of the delayed output edges. Throughout this chapter, we will refer to this procedure as the direct method. Alternatively, our proposed approach is an indirect method: instead of an oscilloscope measuring directly a delay, we use a spectrum analyzer to measure a deliberately generated spur, whose height is in a one-to-one correspondence with the delay to be measured; then, the delay is deduced and employed to calculate the INL. The frequency selectivity of this approach permits to achieve a time resolution up to a few fs.

This chapter is organized as follows. Section 3.2 explains the main idea behind the proposed method. Then, the method is verified by behavioral simulations, as described in section 5.6. In section 3.4, experimental results on a high resolution DTC are presented, while conclusions are drawn in section 3.5 . 


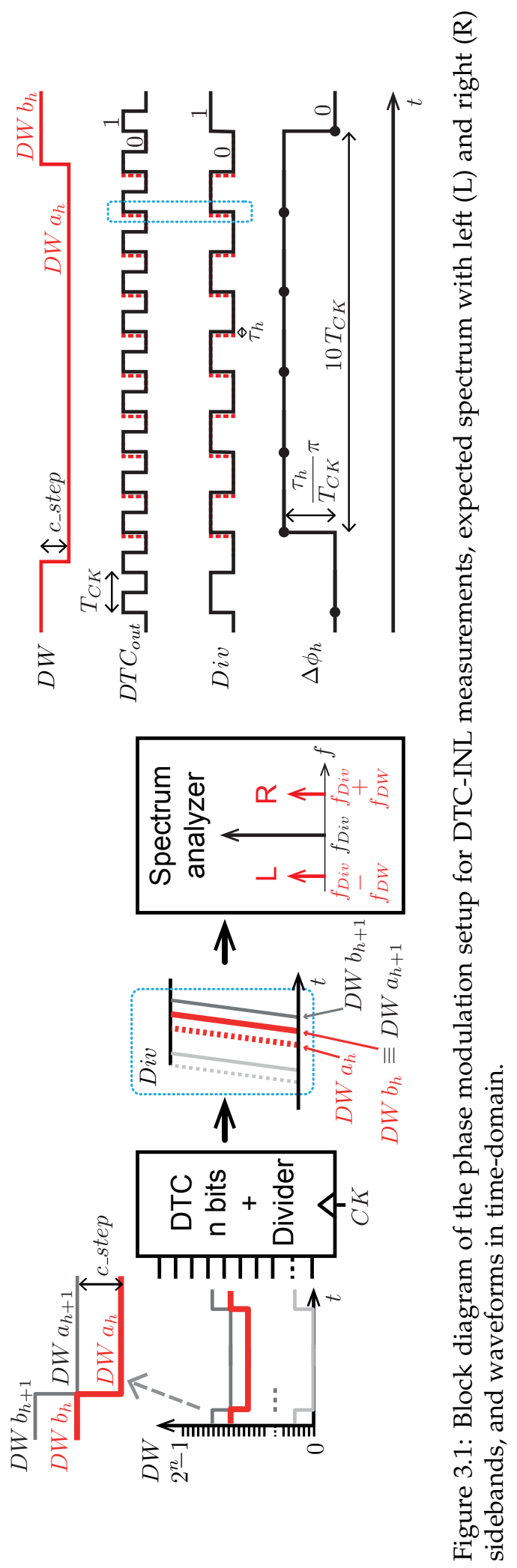




\subsection{Proposed Method}

\subsubsection{The concept}

The main goal of the proposed method is to measure a DTC-INL which is too small to be measured reliably by an oscilloscope. An oscilloscope used for direct delay measurements needs wide bandwidth to avoid affecting the observed rise/fall times of the test signal, leading to high vulnerability to noise and interferences in a broad band of frequencies. In contrast, the method to be proposed relies on phase modulation of the DTC-output by means of a digitally controlled periodic delay-step, that generates a narrowband spur related to the size of that delay-step. This spur can be measured by a spectrum analyzer with small resolution bandwidth, thus avoiding disturbances at all other frequencies.

The concept can be explained using the block diagram and the waveforms shown in Fig. 3.1. The Delay Word (DW) is the code at the digital input of the DTC and is periodically switched between two values: DW $a_{h}$ and $D W b_{h}$ (red waveform). Subscript $h$ will be used to identify a particular starting code used for experiment $h$.

At the DTC output, the rising edges are delayed by a time that depends on the input code. The periodic switching between DW $a_{h}$ and $D W b_{h}$ produces a jump of the rising edge of the DTC output between two determined positions, as shown in Fig. 3.1. As only the edge controlled by the DTC should be detected, and not the other, $\mathrm{a} \div 2$ frequency divider is inserted between the DTC output and the spectrum analyzer. In this way, a phase modulation of the signal Div is achieved. Note that the modulating signal DW (with frequency $f_{D W}$ ) is a code waveform (in DTC LSBs), because of the digital nature of the DTC input. The phase modulation appears, in the frequency domain, as a couple of sidebands, shifted by an offset frequency $f_{D W}$ (and its harmonics) from the carrier frequency $f_{D i v}$. These sidebands can be measured using a spectrum analyzer.

\subsubsection{Analysis}

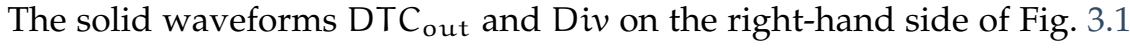
are the unmodulated signals that will occur if the code applied at the DTC input is constantly equal to $D W b_{h}$. The dotted waveforms are the modulated signals: they coincide with the solid waveforms when DW $b_{h}$ is applied, but they are shifted to the dashed edges as long as DW $a_{h}$ is applied.

The frequency $f_{D w}$ of the code waveform is chosen so that the resulting sidebands are located in an interference-free portion of the spectrum, and far enough from the carrier frequency $f_{C K}$ to not be affected by its phase noise, including $1 / \mathrm{f}$ noise. After interference measurements, we chose $f_{C K}=20 f_{D W}$, as shown in Fig. 3.1. However, the following analysis is independent of the choice of $f_{D W}$. 
The waveform $\Delta \phi_{h}$ on the bottom right in Fig. 3.1 represents the phase difference between the unmodulated Div signal (constant DW) and its modulated form (DW square wave with height $c_{\text {step }}$ ), sampled at every rising edge of the Div signal. The waveform $\Delta \phi_{h}$ is a square wave with the same frequency as DW signal; it has $50 \%$ duty cycle and its height is $\tau_{h} /\left(2 T_{C K}\right) * 2 \pi=\tau_{h} / T_{C K} * \pi$, where $T_{C K}$ is the clock period and $\tau_{h}$ is the delay-step produced by the code-step c_step $=D W b_{h}-D W a_{h}$, as shown in Fig. 3.1. The first harmonic $\Delta \Phi_{1 \mathrm{~h}}$ of the phase difference is given by:

$$
\Delta \Phi_{1 \mathrm{~h}}=2 \frac{\tau_{\mathrm{h}}}{\mathrm{T}_{\mathrm{CK}}}
$$

and can be treated as in standard phase modulation theory [70, 71], leading to a spur level relative to the carrier $[\mathrm{dBc}]$ given by $20 \log _{10}\left(\Delta \Phi_{1 \mathrm{~h}} / 2\right)$ and, therefore:

$$
\operatorname{spur}_{h}\left(f_{d i v} \pm f_{D W}\right)=20 \log _{10}\left(\frac{\tau_{h}}{T_{C K}}\right) \quad[d B c]
$$

Equation (3.2) allows the application of a spectrum-analyzer-based time measurement, because it provides the link between the frequency-domain, in which the measurements are actually done, and the time-domain. From the spur measurement, through equation (3.2), we can deduce the measured delay-step $\tau_{h}$ associated with c_step. Next, the associated Differential Nonlinearity (DNL), expressed in seconds, can be calculated with the following:

$$
\operatorname{DNL}(\mathrm{h})=\tau_{\mathrm{h}}-\tau_{\mathrm{id}}
$$

where $\tau_{\text {id }}$ is the ideal delay-step produced by the code difference $c \_s t e p$, evaluated as the average of all the values $\tau_{h}$ obtained. Finally, the INL can be computed from the cumulative sum of the DNL:

$$
\operatorname{INL}(h)=\sum_{k=0}^{h} \operatorname{DNL}(k)
$$

\subsubsection{Sensitivity and Resolution}

To incorporate all DTC-codes in the INL test, the best value to assign to c_step is 1 LSB. However, this choice can result in very slow measurements to get a complete INL plot, because the number of required points is $2^{n}-1$ for an $n$-bit DTC, while a measurement with narrow resolution bandwidth with a spectrum analyzer takes considerable time.

If the INL-behavior of a DTC is rather smooth, its linearity can also be described with a subset of INL points, using a coarser delay-step. However, the logarithmic relation between spur and delay in equation (3.2) suggests that a very coarse delay-step may lead to reduced sensitivity. This is because a spectrum analyzer has a limited resolution and accuracy and at some point the variation of spur strength may be too small to be detected. 


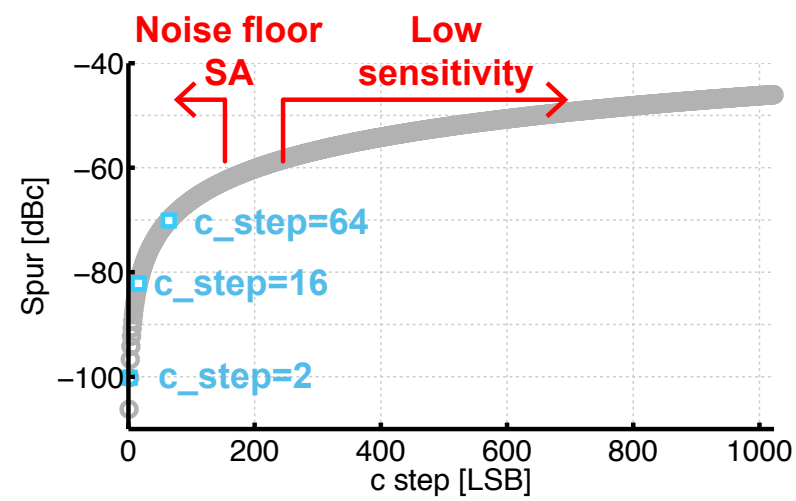

Figure 3.2: Plot of spur as a function of c_step, described in equation (3.6), for the case $\mathrm{n}=10, \tau_{\mathrm{FS}}=100 \mathrm{ps}$, and $T_{C K}=20 \mathrm{~ns}$.

In this section, we will focus on this trade-off between noise-limitations and the limited sensitivity.

The relation between input codes and delays produced by the DTC is linear:

$$
\tau_{h}=\frac{\tau_{F S}}{2^{n}-1} c_{-} \text {step }
$$

where $\tau_{h}$ is the delay-step resulting from the application of the code difference $c_{-}$step at the DTC's input, $n$ is the DTC's number of bits, and $\tau_{F S}$ is the DTC's full-scale delay (i.e. the delay corresponding to the input code going from 0 to $2^{n}-1$ ). Equation (3.2) can be rewritten in the following form:

$$
\operatorname{spur}_{h}\left(f_{d i v} \pm f_{D W}\right)=20 \log _{10}\left(\frac{\frac{\tau_{F S}}{2^{n}-1} c \_s t e p}{T_{C K}}\right) \quad[d B c]
$$

where the logarithmic dependence of $\operatorname{spur}_{h}$ as a function of $c_{-}$step is evident, as shown in Fig. 3.2, for the case $n=10, \tau_{F S}=100$ ps, and $\mathrm{T}_{\mathrm{CK}}=20 \mathrm{~ns}$.

The sensitivity of the method can be quantified as the variation in spur strength due to the change of the delay-step, evaluated at a certain nominal delay-step value. We can calculate it by taking the derivative of equation (3.2), or as a function of c_step (in LSBs), using equation (3.5), obtaining:

$$
\Delta \operatorname{spur}_{\mathrm{h}}=\left[20 \log _{10}(e)\right] \frac{\Delta \tau_{\mathrm{h}}}{\tau_{\mathrm{h}}} \simeq 8.69 \frac{\Delta \mathrm{c} \_ \text {step }}{\text { c_step }}
$$

Equation (3.7) can be used to understand the limits associated with the c_step choice, highlighted in the spur curve in Fig. 3.2.

For a coarse c_step (around 200 LSB), the limitation is the flatness of the spur curve, i.e. a low sensitivity. As an example, with the values used for the plot in Fig. 3.2, for c_step $=200$ a deviation of 3 LSB (non-linearity) in the DTC's delay would result in only $0.13 \mathrm{~dB}$ spur change, which is 
hardly distinguishable from other environmental sources of variation (the experimentally observed uncertainty was $0.2 \mathrm{~dB}$ in the PXA-SA [72]).

For a c_step equal to one or a few LSBs, more INL points are available, but the main limit is the noise floor of the spectrum analyzer that can prevent it from distinguishing the low spur. However, the spectrum analyzer's noise floor can be reduced with a narrow resolution bandwidth RBW (up to $-155 \mathrm{dBm}$ noise floor with $\mathrm{RBW}=1 \mathrm{~Hz}$ in the PXA-SA [72]).

A good value for c_step is, therefore, the minimum value needed to distinguish the spur from the noise floor. For example, with RBW $=200 \mathrm{kHz}$, the spectrum analyzer's noise floor is $-102 \mathrm{dBm}$ for [72]; with a $3 \mathrm{dBm}$ carrier and equation (3.6), the value c_step $=2 \mathrm{LSB}$ would produce a distinguishable spur of $-100 \mathrm{dBc}=-97 \mathrm{dBm}$, with a resulting time resolution of $196 \mathrm{fs}$. An average between multiple measurements is needed to reduce the variability due to noise. By pushing the noise floor to the minimum, it becomes possible to detect a $-140 \mathrm{dBc}$ spur, corresponding (from equation (3.2)) to only $2 \mathrm{fs}$ time delay. This value is far beyond the hundreds of fs values achievable with top-class oscilloscopes commercially available [67].

\subsubsection{Algorithm}

Aiming to detect sub-ps INL, the essence of the proposed measurement method is to always generate the same delay $\tau_{h}$ by changing the input waveform. In this way, the method's sensitivity in equation (3.7) is the same during the measurements, and other variations are minimized. Depending on the spur strength, the measured delay can be higher or lower than its nominal value and this determines the polarity of the INL curve.

The algorithm of the proposed method is based on these considerations together with equations (3.2)-(3.4). It consists of the following steps, partly shown in Fig. 3.1:

1. divide the overall code range into equal intervals of height $c \_s t e p$, covering codes from 0 up to $2^{n}-1$;

2. start with index $h=0$

3. apply the DW waveform $h$ and measure spur $h$;

4. calculate $\tau_{h}$ by applying equation (3.2);

5. increase $h$ and repeat steps 3 to 4 , until all values of $h$ have been considered;

6. evaluate $\tau_{i d}$ as the average of all the values $\tau_{h}$;

7. calculate DNL using equation (3.3) and INL using equation (3.4). 


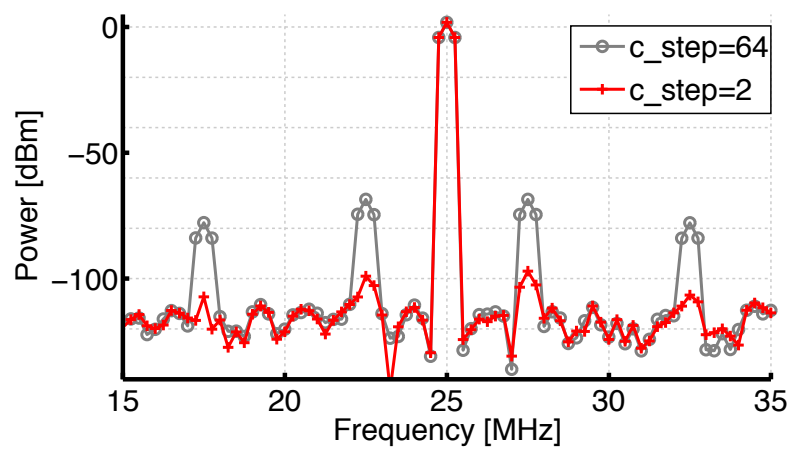

Figure 3.3: Simulated spectrum of Div signal, with c_step $=2$ LSB and c_step $=64$ LSB applied at the DTC input.

Notice that in step 1 the DW waveforms differ only by their lower $\left(D W a_{h}\right)$ and upper $\left(D W b_{h}\right)$ values. Therefore, each waveform is identified by the index $h$. The upper value of one DW waveform must coincide with the lower value of the next one, that is $D W b_{h}=D W a_{h+1}$; this allows us to obtain INL as cumulative sum of DNL.

Ideally, for all the values $h$, the measured spur, and therefore the delaystep $\tau_{h}$, will be always the same. However, due to the circuit nonlinearity, the values $\tau_{h}$ are dependent on the index $h$.

\subsection{Simulations}

We tested the proposed method by running behavioral simulations. The goal is to verify the equations presented in section 3.2 and to check whether the conventional direct and our indirect method lead to the same INL results.

To compare results both in time and in frequency domain, the behavioral simulations need to have enough time resolution, to distinguish the delay of the DTC in the time domain, but also long simulation time, to produce a spectrum with enough frequency resolution. For these simulations, we chose a time resolution of $20 \mathrm{fs}$ and a simulation time of $4 \mu \mathrm{s}$, corresponding to a frequency resolution of $250 \mathrm{kHz}$.

The simulated DTC has $\mathrm{n}=10$ bits, a full-scale delay $\tau_{\mathrm{FS}}=100 \mathrm{ps}$, and a clock with period $\mathrm{T}_{\mathrm{CK}}=20 \mathrm{~ns}$. A quadratic nonlinearity is inserted on purpose in the model with maximum INL of $250 \mathrm{fs}$. The frequency of the $D W$ waveform is set to $f_{D W}=2.5 \mathrm{MHz}$, the Div signal has $f_{D i v}=25 \mathrm{MHz}$. White noise has been added to model a $-120 \mathrm{dBm}$ spectrum analyzer's noise floor.

Fig. 3.3 shows the simulated spectrum of the divider output waveform, as it would appear on the screen of a spectrum analyzer, for two quite different values of c_step within the limits discussed in section 3.2.3, to clearly show the spur differences. As expected, the spectrum exhibits the 


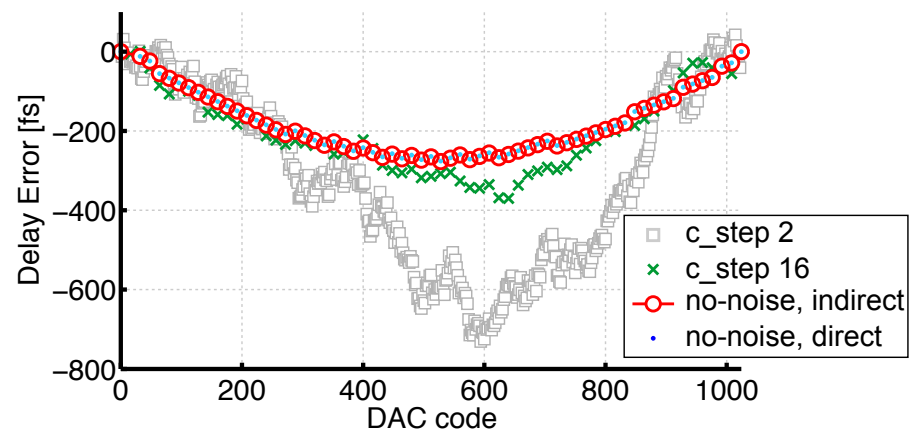

Figure 3.4: Simulated INL, for different values of c_step and for direct and indirect methods. The values of c_step used are highlighted on Fig. 3.2.

carrier tone at $f_{D i v}=25 \mathrm{MHz}$ and two sidebands at $f_{D i v} \pm f_{D W}$ (first harmonics), namely $22.5 \mathrm{MHz}$ and $27.5 \mathrm{MHz}$. For c_step $=64 \mathrm{LSB}$, the figure also shows the third harmonics at $f_{D i v} \pm 3 f_{D W}$, due to the square wave shape of the modulating signal; these higher harmonics do not add more information and they are not considered for the measurements. The simulated first-harmonic sidebands are $-100.6 \mathrm{dBc}$ for c_step $=2 \mathrm{LSB}$ and $-70.4 \mathrm{dBc}$ for $\mathrm{c}_{-}$step $=64 \mathrm{LSB}$, matching the values obtained from equation (3.6).

Fig. 3.4 aims to compare the INL using both the direct and the indirect methods. The figure also shows the effect on the INL curve by two c_step values in the trade-off range discussed in section 3.2.3. For simplicity, the two methods are compared in the noiseless case, with c_step $=16$ LSB. The shape of the noiseless INL curve (circles) exhibits the quadratic nonlinearity inserted on-purpose in the behavioral model. The algorithm of section 3.2.4 was used to calculate it, where one spectrum for each point of the INL curve has been obtained. The small-dotted curve in Fig. 3.4 refers to the direct method. It has been derived by plotting the DTC output as a function of time, then evaluating the time instants $t_{k}$ where the rising edges cross a $700 \mathrm{mV}$ voltage threshold and finally using the standard formulas from [73]. The INL from the two methods differ by at most $1.1 \mathrm{fs,}$ due presumably to numerical noise, i.e. only $1 \%$ of 1 DTC LSB.

By adding $-120 \mathrm{dBm}$ white noise in the simulations, we can investigate the effect of different code-step choices on the INL. For c_step $=2$ LSB, the first spur is close to the simulated noise floor, as shown in Fig. 3.3, resulting in a detailed (511 points) but noisy INL plot in Fig. 3.4. The drift due to high-frequency noise can be reduced by obtaining multiple INL plots and averaging them at each code. However, as simulations already take multiple days, no averages for any value of c_step have been done here. Instead a higher value of c_step $=16$ LSB was used, resulting in a less noisy result closer to the true INL. A larger c_step produces less INL points but is more robust to noise. 


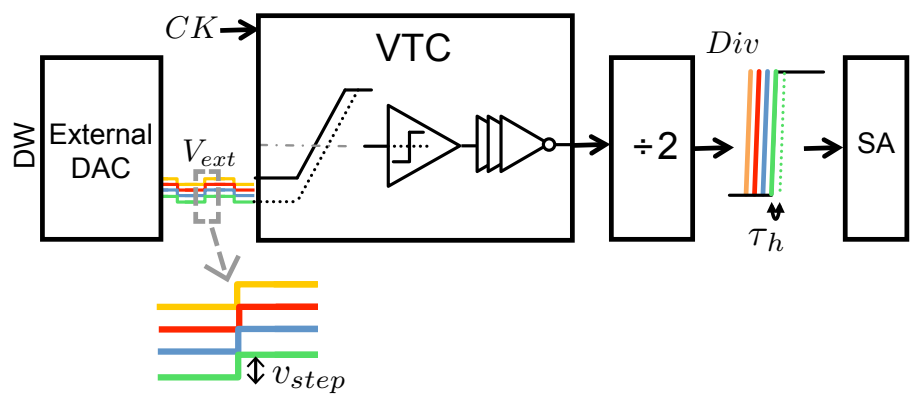

Figure 3.5: Setup used to measure the INL of the DTC [CP:2] (acting as a VTC), with the proposed method.

\subsection{Measurements}

We actually developed this INL-measurement method to allow for measuring the INL of a record high-resolution DTC, implemented in CMOS $65 \mathrm{~nm}$ technology that exploits a constant slope principle [CP:2] . Measuring the DTC with on-chip DAC using the direct method failed, because of the DTC resolution in the order of tens of fs. Fig. 3.5 shows the block scheme of the measurement setup. The DTC realizes a delay using a ramp waveform with a fixed slope, starting from an initial voltage defined by a Digital-to-Analog Converter (DAC). The delay is controllable by the DAC voltage, which can either be on-chip or external. The digital interface of this chip is not fast enough to support $\mathrm{MHz}$ modulation of the DAC code. Instead, an external DAC (Agilent M8190A Arbitrary Waveform Generator) was used in these experiments to produce a square wave $\left(V_{e x t}\right)$ that periodically switches between two voltage levels, effectively implementing the delay-step, and the chip acts as a Voltage-to-Time Converter (VTC). The procedure is the same as described in section 3.2; compared to the scheme in Fig. 3.1, the only difference here is that the DW waveforms with height c_step are now converted into $V_{\text {ext }}$ square waves with height $v_{-}$step, through the external DAC. The $V_{\text {ext }}$ jitter did not affect the sideband position during the measurement. We used the 10 MSB-bits of the Agilent M8190A 14-bit DAC. We checked its INL was below $+/-0.5$ LSB $(0.05 \%$ referring to 10 -bit full-scale) so it is not the bottleneck in our DTC-INL measurement. Data averaging can reduce the DAC thermal noise, however some $1 / \mathrm{f}$ noise remains.

The measurements are done with $\mathrm{f}_{\mathrm{CK}}=50 \mathrm{MHz}, \mathrm{f}_{\mathrm{Div}}=25 \mathrm{MHz}$, and using 40 values for the square waves $V_{\text {ext }}$ with $f_{V_{\text {ext }}}=f_{D W}=2.5 \mathrm{MHz}$ and height $v_{-}$step $=0.98 \mathrm{mV}$ each. The choice of $v_{-}$step is equivalent to c_step of about 25 LSB in a 10-bit full scale, on the same order as the c_step choices in section 5.6. A smaller $v \_s t e p$ or c_step would result in more measured INL points but is less robust to noise and requires longer measurement time. The algorithm in section 3.2.4 is repeated 50 times, leading to 50 sweeps through the set of 40 square wave voltages. The DTC 


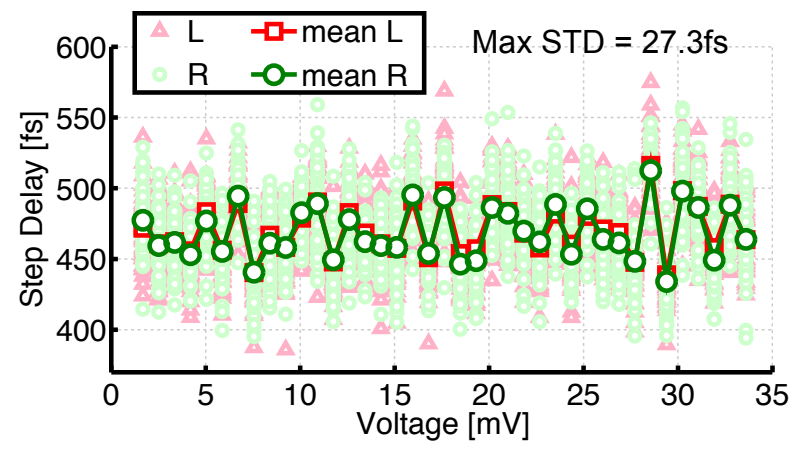

Figure 3.6: Measured delay-step produced by the DTC [CP:2] , as a function of the upper voltage of the modulating square waves; 50 repetitions for each delay.

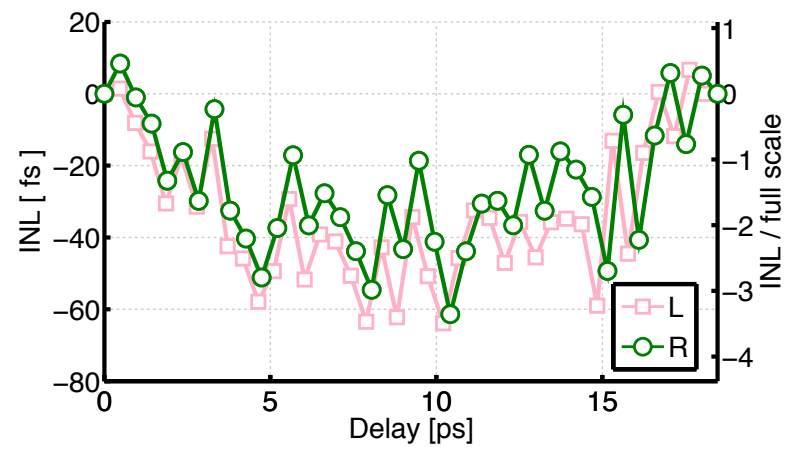

Figure 3.7: Measured INL, produced by the DTC [CP:2], set with fullscale $19 \mathrm{ps}$, using the proposed method ( $\mathrm{V}_{\text {ext }}=0$ to $33.6 \mathrm{mV}$ with steps of $0.98 \mathrm{mV})$.

in measurement has a tunable full-scale delay $19-189 \mathrm{ps}$, and we use it here at its minimum delay to apply the method in the most challenging case. The spectrum analyzer's resolution bandwidth is set to $10 \mathrm{kHz}$, leading to $-115 \mathrm{dBm}$ noise floor and $-118 \mathrm{dBc}$ minimum detectable spur (assuming $\mathrm{SNR}=0 \mathrm{~dB}$ and $3 \mathrm{dBm}$ carrier), and resulting in $25 \mathrm{fs}$ time resolution from equation (3.2).

Fig. 3.6 shows the 40 measured delays and indeed shows an average of about $475 \mathrm{fs}$ ( $19 \mathrm{ps} / 40)$. The delays have been calculated separately for the left $(\mathrm{L})$ and the right $(\mathrm{R})$ sideband. The maximum standard deviation is $27.3 \mathrm{fs}$. The resulting averaged INL curve is shown in Fig. 3.7. The two $\mathrm{y}$-axes refer to the absolute INL in fs, and its normalized value with respect to the DTC full-scale delay, respectively. The maximum INL value is $64 \mathrm{fs}$ and corresponds to $0.34 \%$ in the normalized scale. The difference between left and right sidebands produces a maximum INL difference of $20 \mathrm{fs}$, that is less than the standard deviation $27.3 \mathrm{fs}$ of the time step measurement. Therefore, in this case, either the left or the right spurs produce a sufficiently 
precise INL plot. However, depending on the implementation of the DTC, if there is also a coexisting amplitude modulation, the delay measured using the left spur would be higher than the one for the right spur. In such a case, averaging between left and right spurs produces the proper result.

\subsection{Conclusion}

In this chapter, we have presented a very sensitive method to measure the INL of DTCs, based on phase modulation, and capable to achieve a time resolution of a few fs, which is 1-2 orders of magnitude better than what is achievable with high-speed sampling oscilloscope. The new method has been verified with behavioral simulations, and used to measure the INL of a high-resolution DTC with $19 \mathrm{ps}$ full-scale. An INL in the order of $50 \mathrm{fs}$ was measured, with a standard deviation of $27.3 \mathrm{fs}$. 


\section{Pulse-Output DFCs: reducing spurs with a}

\subsection{Introduction}

Square waves or pulse-output clock signals are needed as timing references in many applications like digital clocks, samplers, and data converters [73, 74], Local Oscillators (LOs) for hard-switched mixers [75, 76] reference clocks in e.g. phased arrays [77] and clock recovery [78]. Furthermore, obtaining precise $50 \%$ duty-cycle is often important [79], e.g. for evenorder harmonic suppression [80] or in cases where dividers are used to generate multi-phase clocks [81].

High frequency pulse-output synthesizers with a digitally programmable frequency commonly employ Phase-Locked Loops (PLLs) [11]. However, Direct Digital Synthesizers (DDSs) [15] or a hybrid combination between DDS and PLL is also gaining interest [82, 83], especially in the context of Systems-on-Chip (SoCs), due to the DDS wide frequency range, extremely fine frequency resolution, immediate and phase-continuous frequency switch and compatibility with digital design flows. A key benefit for SoCs is that only one simple integer-N PLL is needed to be integrated on-chip to produce a high-frequency system clock, while multiple DDS blocks can generate from it all the other frequencies required in the system, in a flexible way. This solution is area-efficient and avoids coupling issues like in multi-PLL analog solutions, typically originating from coupling between resonating tank components used in the Voltage-Controlled Oscillators (VCOs) of PLLs running at different frequencies [75].

Direct digital frequency synthesis techniques are reviewed in [7], where two types are distinguished (Fig. 4.1): Digital-to-Period Converters (DPCs) and Digital-to-Frequency Converters (DFCs). DPCs generate a time-averaged

This chapter has been previously published in [CP:3] . 


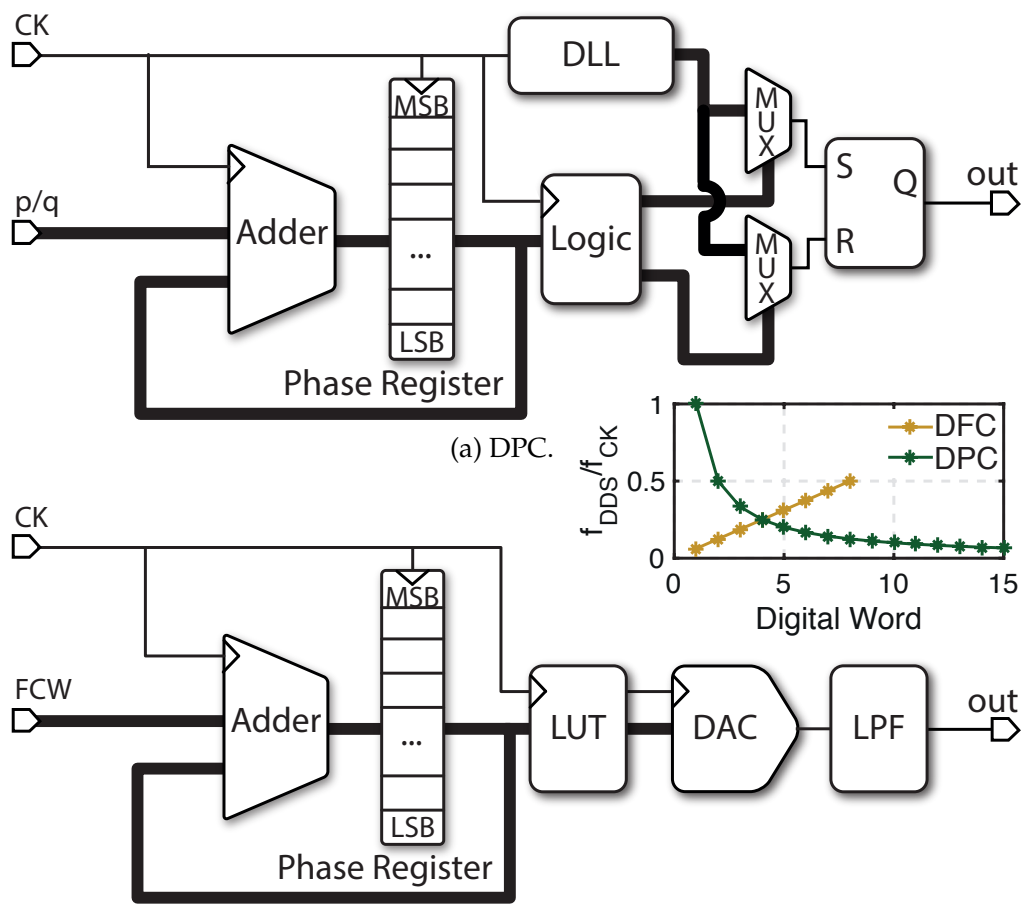

(b) Sine wave DFC.

Figure 4.1: Block scheme of a DPC [44] and traditional sine wave DFC [84]. The phase register synchronizes the current adder output to the clock. MSB and LSB stand for most/least significant bit of the adder output. The graph shows the output frequency grid for a 4-bit input word $\mathrm{p} / \mathrm{q}$ (DPC) and FCW (DFC).

period proportional to their digital input, while DFCs produce a timeaveraged frequency proportional to their input code. For some applications it is convenient to control period, but for others digital frequency control is preferred, e.g. because of the linear instead of hyperbolic control function to the output frequency and ease of direct frequency modulation.

When starting from a fixed system clock, generating other period times or frequencies unavoidably comes with deterministic timing errors, often referred to as deterministic jitter, that appear in the output spectrum as spurious tones (spurs).These tones are potential causes of adjacent channel interference in multi-channel wireless communications systems [85] and degrade the dynamic range in data converters [86]. Thus, frequency synthesizers are often benchmarked in terms of maximum spur, e.g. fractional-N PLLs [87-89].

In the context of Digital Frequency Synthesis, Delay-Locked Loops (DLLs) have been proposed in the past for deterministic jitter correction [42, 90, 91], but recently Digital-to-Time Converters (DTCs) with sub-picosecond resolution and linearity have become available [58, 92], so they can be used for this purpose. 
DTCs for spur correction have been proposed inside DPC systems [28, $44,45,93]$, while in this chapter we introduce a DTC in a DFC architecture, targeting $50 \%$ duty-cycle pulse-output. We will analyze the DTC specifications (resolution, linearity) that impact the output-spectrum purity and derive simple equations to predict the maximum spurs, to facilitate the system design. Behavioral simulations will be used to prove the mathematical analysis.

Before doing so, we will give a brief overview of the literature in this field in Section 4.2. Section 4.3 explains the DFC system architecture and identifies some DTC features to improve the output spur level and speed. Section 4.4 provides a detailed analysis of the output spurs, relating the DTC impairments to the output spectrum. In that section, the analysis is also validated by comparing it with behavioral simulations. Finally, conclusions are drawn in Section 4.5.

\subsection{Brief overview on DPCs and DFCs}

In a DPC (Fig. 4.1a), the control of the output period is accomplished by adding a continuously incremented delay to the edges of a fixed reference clock [25, 28, 94-96]. The most recent versions of DPCs, like the one shown in Fig. 4.1a, start with a single-phase reference clock, from a fixed-frequency PLL, and employ delay elements (usually DLL+multiplexer) to produce and select the output phase $[42,95]$.

DFCs were the first type of DDSs to be implemented [1,97]. Sine wave DFCs (Fig. 4.1b) with a counter, a Look-Up-Table (LUT) with sine wave samples, a Digital-to-Analog Converter (DAC) and reconstruction filter are probably the best known DFCs $[15,84]$. The counter acts as a digital phase accumulator controlled by a Frequency Control Word (FCW). The average frequency of the overflow bit is the target output frequency, and the multi-bit DAC interpolates to obtain a voltage sine wave. If a 1-bit output (often called pulse-output [15]) is wanted, this can also be done by a DTC. In [43], the potential of a digital delay element inside a DFC producing square waves was recognized and an algorithm was proposed to correct deterministic jitter, based on a DLL implementation.

By comparing a DFC with a DPC, one distinction is their application domain as mentioned above. However, it is also instructive to understand the different nature of their operation and, for their DTC-based implementations, the different role of the delay element. In a DPC the delay element is used to modify the period to adjust the output frequency $(=1 / \mathrm{T})$. In contrast, in a counter-based DFC the average frequency already has the right (average) value [22], and the role of the delay element is the correction of the deterministic jitter, bringing the instantaneous frequency (not simply the time-averaged one) to the target. This ideally cancels the spurs in the output spectrum, thus acting as filtering (hence the name "time-filtered square wave" in [43]). 


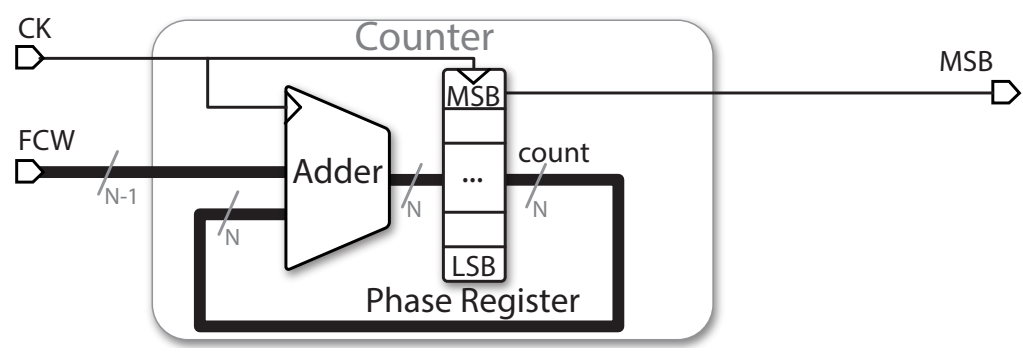

Figure 4.2: Counter-based Pulse-Output DFC.

Consider now the digitally-controlled delay element. In most of the aforementioned works, it has been implemented with a DLL plus multiplexer $[42,90,91,98]$. However a DTC is substantially different from a DLL. A DLL requires two clock edges, between which the output edges are interpolated. It produces multiple outputs, i.e. a set of available edges for the multiplexer to choose from. Instead, a DTC is a single-shot device that only produces the edge that is needed, not wasting power in unused ones. Furthermore, a DTC acts in open loop, while a DLL has a feedback loop for delay control.

A DLL is typically limited to $5-6$ bits for a reference input clock of $1 \mathrm{GHz}$, as the delay of each buffer is typically limited to one gate delay ( $10 \mathrm{ps}$ in $65 \mathrm{~nm}$ technology). This resolution limits the output spectrum purity of digital synthesizers and additional spur suppression techniques are usually added. In [42], a 5-bit DLL produces maximum spurious tones of $-24.5 \mathrm{dBc}$ for an output frequency of $997 \mathrm{MHz}$, that can be pushed down to $-48.2 \mathrm{dBc}$ using dithering. The DDS in [23] achieves $-65 \mathrm{dBc}$ spurs for an output frequency of $496 \mathrm{MHz}$ using a second order $\Sigma \Delta$. Vernier delay lines could be another alternative to DLLs [99], but they produce more edges than actually used, not being power efficient.

Recent research in DTCs for low-power fractional PLLs [33, 50, 100] has led to substantial improvements in DTC performance and has boosted the development of high-speed implementations at $\mathrm{GHz}$ frequencies that are suitable for use in DDS systems [28, 58, 92]. Most of these DTCs exploit a constant-slope delay generation [CP:2] and achieve more than 10-bit resolution, a few LSB INL and a FoM down to a few fJ/conversion.

Choosing a DTC for deterministic jitter correction not only improves the output spectrum of the synthesizer, due to the $100 \times$ increased resolution compared to the DLL, but also has a different impact on the output spectrum due to the different sources of DTC impairments, that this chapter aims to analyze. 


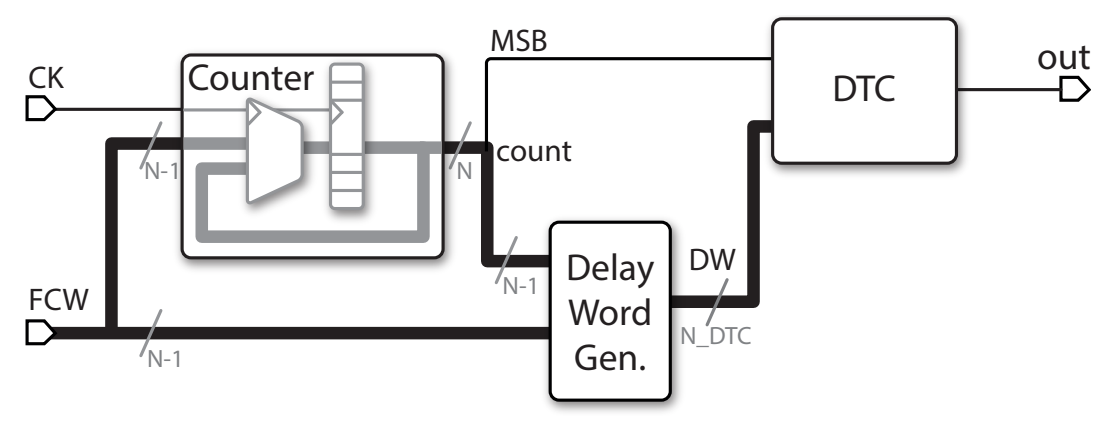

Figure 4.3: Proposed Pulse-Output DFC retimed by a DTC.

\subsection{Proposed Architecture}

\subsubsection{Pulse-Output DFC}

The core of the system proposed and analyzed in this work is the PulseOutput DFC in Fig. 4.2, also known as Pulse DDS [15]. This DDS concept has been known for decades $[3,4]$ and is based on the overflow rate of a binary counter with programmable step-size, named Frequency Control Word (FCW). The counter, acting as phase accumulator, consists of an $\mathrm{N}$-bit adder and an $\mathrm{N}$-bit register, thus the operation is modulo $2^{\mathrm{N}}$. The MSB of the counter is a square wave that has on average the target output frequency [22]:

$$
f_{D D S}=\frac{f_{C K}}{2^{N}} \cdot F C W, \quad 0 \leqslant F C W \leqslant 2^{N-1}
$$

Where $f_{D D S}$ is the average synthesized frequency of the Pulse-Output DFC, and $f_{C K}$ is the (fixed input) clock frequency. The maximum achievable value of $f_{D D S}$ is therefore $f_{C K} / 2$. The frequency step $f_{C K} 2^{-N}$ can be improved by increasing the size $\mathrm{N}$ of the counter. Based on (4.1), a DFC can also be seen as a programmable frequency divider [84].

\subsubsection{DTC for edge retiming}

The counter's MSB in the Pulse-Output DFC in Fig. 4.2 suffers from timing irregularities due to the coarse phase increment of the accumulator. To correct them, the MSB edges can be re-timed with a DTC, as shown in the proposed architecture in Fig. 4.3. The least-significant bits of the counter are used to compute the DTC delay word DW. In this architecture, the DTC acts on both rising and falling edges on its input waveform MSB, to obtain a $50 \%$ duty-cycled square wave at the output. The correction mechanism is illustrated in the time domain in Fig. 4.4, for the case $\mathrm{N}=4$. The MSB of a counter with programmable step-size FCW is shown over time, together with the total counter output, which is updated every clock period $\mathrm{T}_{\mathrm{CK}}$. When FCW is not a power of 2, the coarse counter's increment causes the MSB edges to have a periodically incorrect timing, compared to 


\section{0}

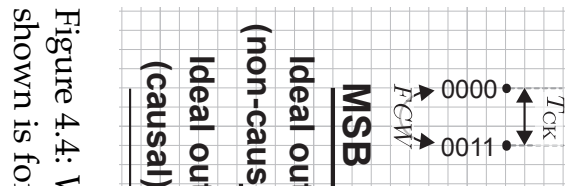

윽

$\| \stackrel{0}{0}$

这灵

묵

11 을

$\omega$

꾸 $\stackrel{2}{2}$

$\sum \exists$

||

แ $\nabla$

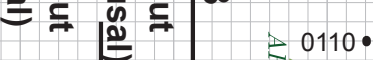

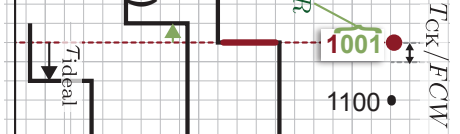

${ }_{1} 1111 \bullet$

$\square^{0101}$

$\square^{-1000 \bullet} \frac{1011 \bullet}{1110 \bullet}$

1

2

ợ

is

ڤี

華

ㄱ.

:

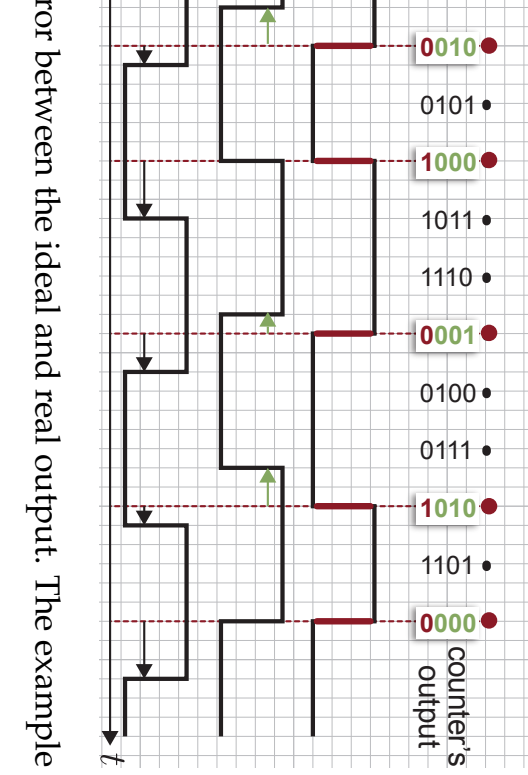


the ideal output (second signal in Fig. 4.4). These periodic errors, together with the square waves not being $50 \%$ duty-cycled on short term, give rise to spurs in the output spectrum [84].

The elegant correction algorithm proposed in [43] for a DFC with Digital Delay Line (DDL) can also be applied here to calculate, at every MSB edge, the DTC delay. The algorithm is based on the observation that the counter residue $A R$ (i.e. the counter output excluding its MSB), read out at each edge of the waveform MSB, contains the excess error compared to the corresponding ideal non-causal edge. This can be seen in Fig. 4.4. The signal ideal out is the DTC output from Fig. 4.3. The grid pitch in Fig. 4.4 is $T_{C K} / F C W$ which is the equivalent time for the counter to increase its output by one. The edge positions of signal ideal out (non-causal) are AR pitches before the corresponding edges of MSB. The output waveform is called non-causal as it would be produced by adding negative delays to the edges of MSB. In [43] it was proposed to make the output causal by adding a clock period to all the output edges. Thus, the net positive delay added to each MSB edge to produce the causal ideal output (black rightward arrows in Fig. 4.4) is

$$
\tau_{\text {ideal }}=(\mathrm{FCW}-\mathrm{AR}) \frac{\mathrm{T}_{\mathrm{CK}}}{\mathrm{FCW}}
$$

Where counter residue $A R$ is bounded: $0 \leqslant A R \leqslant F C W-1$. The maximum required delay to be added (i.e. the programmable delay range needed) is $\mathrm{T}_{\mathrm{CK}}$.

While digital correction schemes are more versatile and easier to build than their analog counterparts [21,22], they can only provide quantized and hence approximated values of (4.2). If $\mathrm{N}_{\mathrm{DTC}}$ is the number of bits of a DTC with full-scale delay $T_{C K},(4.2)$ can be rewritten as function of the DTC time resolution $\mathrm{T}_{\mathrm{CK}} / 2^{\mathrm{N}_{\mathrm{DTC}}}$ as

$$
\tau_{\text {ideal }}=\left[(F C W-A R) \frac{2^{N_{\text {DTC }}}}{F C W}\right] \frac{T_{C K}}{2^{N_{\text {DTC }}}}
$$

Where the term in square brackets is the current delay word.

\subsubsection{Pushing down spur levels with a DTC}

This section considers design aspects of the Pulse-Output DFC + DTC in Fig. 4.3, with focus on the output spur levels and frequency. These considerations will lead to a set of parameters describing the high-level behavior of the DTC, that will be used in the behavioral modeling explained in Section 4.4.

As mentioned before, spur levels depend on the quality of the DTC. Besides the quantization errors, the DTC Integral Non-Linearity (INL) is also crucial for the output spurs. In fact, it is directly related to the harmonic distortion [74], since it implies a deviation of the DTC transfer curve from the straight line. The DTC DNL, instead, is less important here. Assuming 


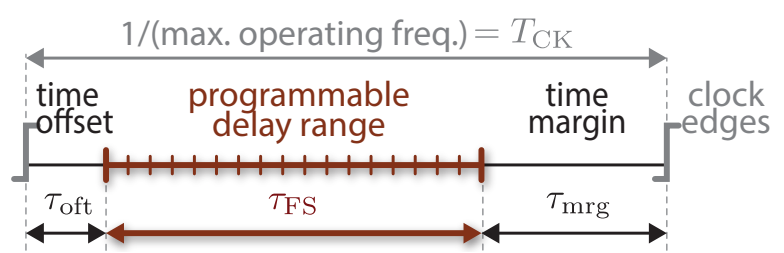

(a) Time slots for a single DTC.

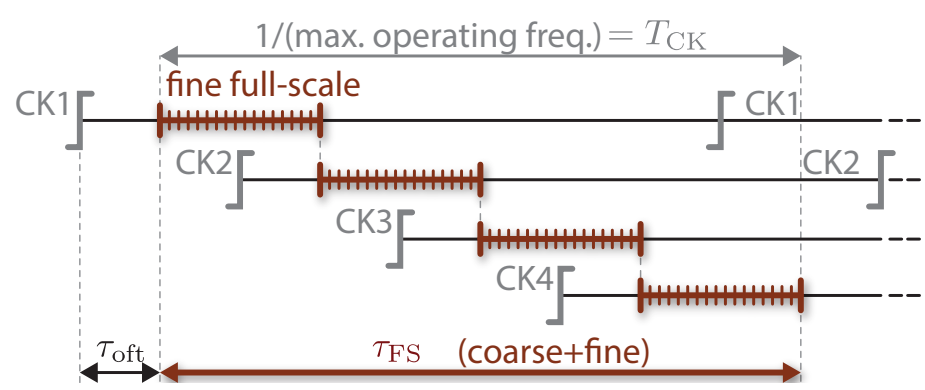

(b) Coarse + fine delay scheme.

Figure 4.5: Achieving a full programmable DTC delay range with dividers. Example based on the four-phase generator in [81].

it is divided into correlated and uncorrelated parts [73], its uncorrelated fraction can be considered as quantization error (section 4.4.1), while its correlated fraction summed up is the main source of the INL, so it is taken into account with it (section 4.4.4).

The DTC maximum operating frequency is $1 / \mathrm{T}_{\mathrm{CK}}$ (when the waveform MSB changes at every clock edge), and it should provide a programmable delay range of $\mathrm{T}_{\mathrm{CK}}$. However, a practical DTC has a non-zero time-offset, as shown in Fig. 4.5a, and needs a time margin after operation before it is ready for use again. Hence, its programmable delay-range (full-scale) is less than $\mathrm{T}_{\mathrm{CK}}$. To address this DTC limitation, we will consider in the model developed in this work a coarse/fine delay scheme, obtained with frequency dividers, as shown in Fig. 4.5b. Generating four clock phases using dividers by 2 as in [81] allows a DTC time-margin which should be sufficient for most DTC implementations, as shown in the time allocation in Fig. 4.5b. The coarse delay is provided by selecting one clock phase, while the fine delay is generated by the DTC, which is clocked by the selected phase. In this way, the fine full-scale required for the DTC is lowered to $\mathrm{T}_{\mathrm{CK}} / 4$. A calibration will be needed to guarantee that the total programmable delay range $T_{C K}$ will be covered continuously between the coarse and fine mechanisms.

Since the fastest DTC implementations work at a couple of $\mathrm{GHz}$ [58, 92], the system would produce square waves controlled on both edges at maximum $1 \mathrm{GHz}$. The output frequency can be pushed further with timeinterleaving, where different DTCs work in succession. To cover this option in our model, the simplest form of time-interleaving - with only two DTCs 


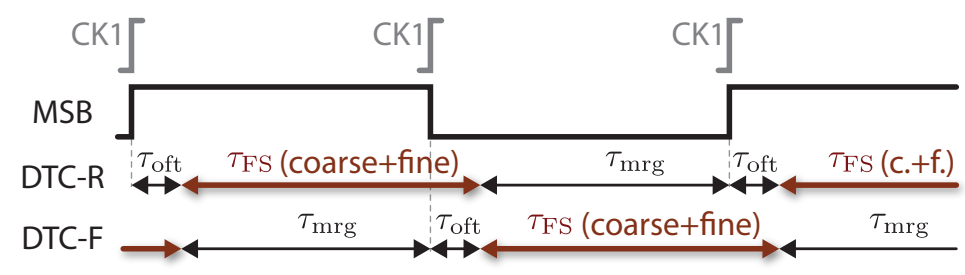

Figure 4.6: Two time-interleaved DTCs for increased maximum output frequency.

- will be considered, as shown in Fig. 4.6. Note that the number of timeinterleaved blocks chosen has no relation with the number of clock phases for the coarse delay. In fact, the time-interleaving technique separates two consecutive full-scale delay-ranges (see, for example, the red arrows for DTC-R (Rise) in Fig. 4.6), but this choice is independent from the way these delay-ranges are internally sub-divided (Fig. 4.5b). With two DTCs alternating at every MSB edge, one DTC happens to be associated with MSB rising edges, the other one with falling edges. For this reason, the two DTCs will be named DTC-R (Rise) and DTC-F (Fall). As additional benefit, time-interleaving can result in extra time margin between the conversion and the next triggering edge, allowing for a better DTC settling to reduce memory effects that would otherwise degrade its INL [CP:2] [50]. In terms of dynamic power consumption, two interleaved CMOS DTCs are almost equivalent to a single DTC operating at double frequency. However, a gain mismatch between interleaved DTCs would produce different delays, whose effect on the output spectrum will be discussed in Section 4.4.

\subsubsection{DTC-based Pulse-Output DFC}

The block scheme of the Pulse-Output DFC in Fig. 4.3 can be detailed based on the features described in the previous paragraphs, resulting in Fig. 4.7. The DTC block in gray now has a coarse/fine structure and contains a R(ise)- and F(all)-DTC. The fine DTCs can be any high-speed implementation discussed in Section 4.2. ${ }^{\dagger}$

The four clock phases $\mathrm{CK}_{1}-\mathrm{CK}_{4}$ are derived from a reference signal with doubled frequency: $f_{C K}=f_{\text {ref }} / 2$. The $\mathrm{N}$-bit counter (phase accumulator) is clocked by $\mathrm{CK}_{1}$. A logic block clocked by $\mathrm{CK}_{4}$ computes the delay word DW from (4.3). The counter's most significant bit $\mathrm{MSB}_{\mathrm{C}}$ is resampled multiple times with $\mathrm{CK}_{4}$ to match the delay of the logic block. Thus, the resulting signal MSB is synchronized with DW. Out of the $\mathrm{N}_{\mathrm{DTC}}=$ $\mathrm{N}-1$ bits of DW, the two most significant bits control the coarse delay:

\footnotetext{
$\dagger$ Whatever the delay mechanism of the DTC is, e.g. constant-slope charging of a capacitor starting from a variable start voltage [58,92], variable current charging $[21,22,24]$ or a variable capacitive load [28], all DTCs can be characterized by their number of bits, INL and full-scale delay, as used in Fig. 4.7. The particular DTC implementation does however impact the physical causes for INL but that goes beyond the scope of this chapter.
} 

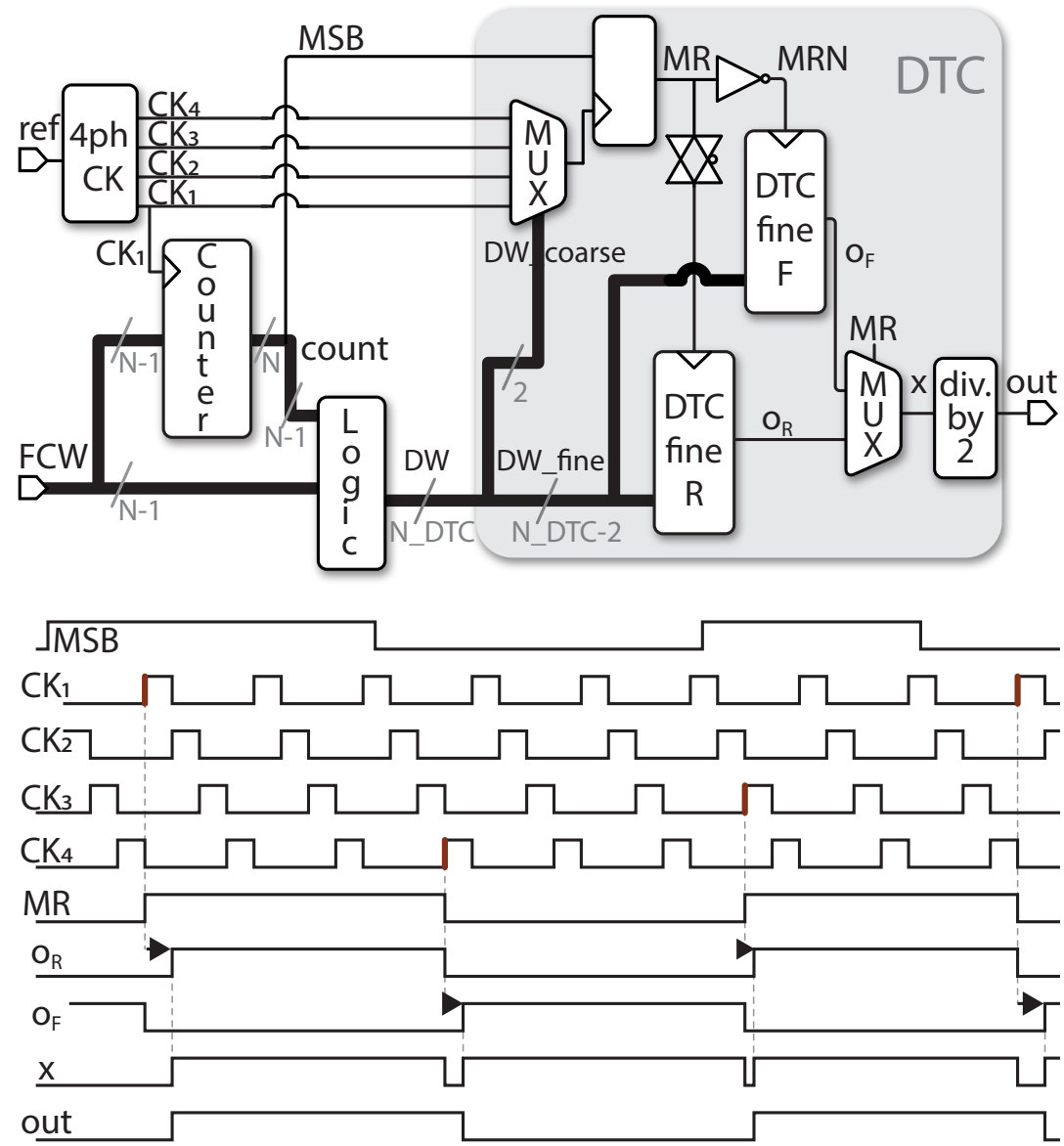

Figure 4.7: Detailed model of the proposed DTC-based Pulse-Output DFC, with the DTC in gray.

they select the correct clock phase $\mathrm{CK}_{1}-\mathrm{CK}_{4}$ to resample $\mathrm{MSB}$, in order to produce the signal MR (resampled MSB). The remaining $N_{D T C}-2$ bits of DW determine the fine delay. They are sent to the two interleaved DTCs, activated by $M R$ and its inverted form $M R N$. The outputs $o_{R}$ and $o_{F}$ of the two interleaved DTCs are then combined to produce the output signal out, as shown in Fig. 4.7. A delta-sigma modulator before the counter would change the profile of the output spurs, thus relaxing the resolution and linearity specifications of the DTC for spur correction. However, in this chapter we will study the effect of a DTC as the only aid for spur canceling, in a deterministic, instantaneous way, to investigate how good is the DTC correction before resorting to orthogonal techniques. 


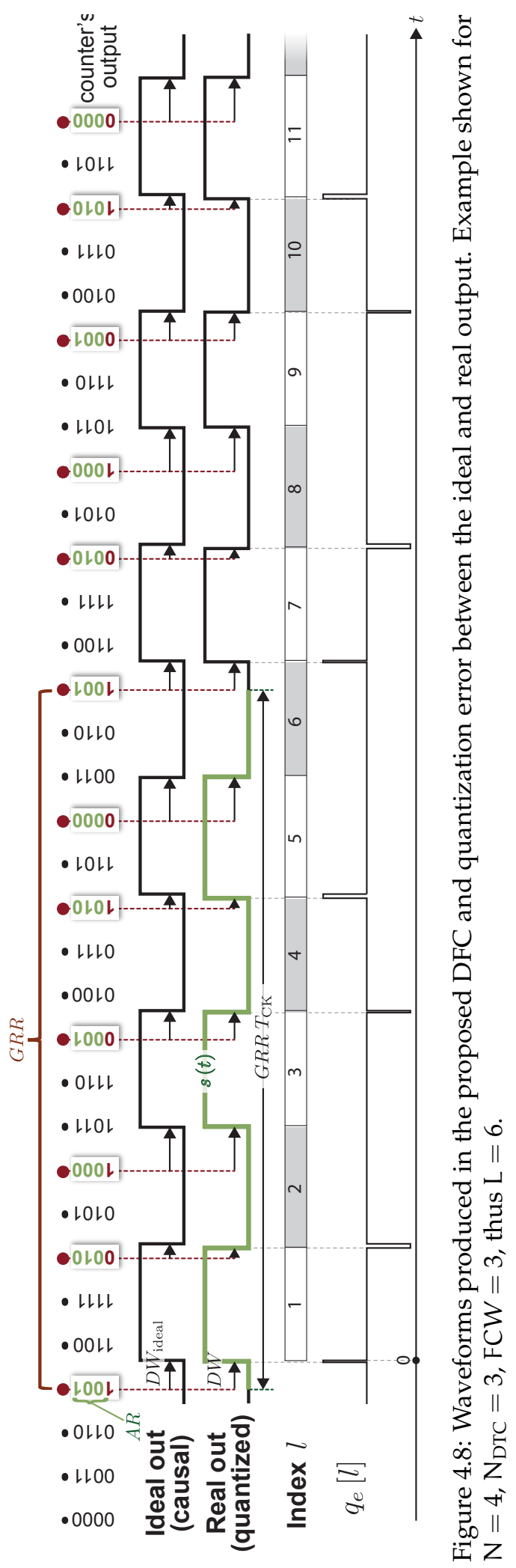




\subsection{Output spectrum in a DTC-based DFC}

The output spectrum of the proposed DFC in Fig. 4.7 will be analyzed, focusing on spurs produced by deterministic errors and on the effect of the DTC impairments on the Spurious-Free Dynamic Range (SFDR). The reference clock is assumed jitterless since it can be produced by a low noise integer-N PLL $[59,101]$ and its frequency is divided by the DFC logic, thus further reducing its impact on phase noise [102]. If the goal is to produce square waves with $50 \%$ duty-cycle, the output's odd harmonics should not be considered in the evaluation of the SFDR (they are part of the wanted signal). We will first investigate the effect of the DTC number of bits $\mathrm{N}_{\text {DTC }}$ (quantization error), while the DTC impairments will be analyzed in Section 4.4.4.

\subsubsection{Effect of DTC quantization error on the output spectrum}

Assume that the counter in Fig. 4.7 is reset to zero as initial state. Its finite length $\mathrm{N}$ implies its output is calculated modulo $2^{\mathrm{N}}$. The number of steps required to make the counter return to its starting value is the numerical period of the counter's output and is often called Grand Repetition Rate (GRR) [84]:

$$
\mathrm{GRR} \triangleq \frac{2^{\mathrm{N}}}{\mathrm{GCD}\left(2^{\mathrm{N}}, \mathrm{FCW}\right)}
$$

Where GCD (.) denotes the greatest common divisor. If $f_{C K}$ is the frequency of the counter's clock, then the repetition frequency of the phase register is $f_{C K} / G R R$.

The output spurs are residual errors coming the correction of the counter's MSB and therefore they are placed at integer multiples of the counter's repetition rate $f_{C K} / G R R$. The output frequency of the DFC can also be expressed as a function of this rate, from (4.1) and (4.4):

$$
f_{D D S}=\frac{f_{C K}}{G R R} \cdot \frac{F C W}{G C D\left(2^{N}, F C W\right)}
$$

In this section we will show that the sub-harmonics, i.e. the spurs located at frequencies between DC and $f_{D D S}$, are related to the Discrete Fourier Transform (DFT) of the DTC quantization error. The analytical procedure follows similar steps as the analysis for DPCs [44, 93], targeted on finding a sequence that models the output quantization error. However, since the boundary conditions of DPCs and DFCs are different, as will be shown in Table 4.1 and in Fig. 4.11, the two quantization sequences do not share the same symmetries, except for special cases where sequences are identical.

Fig. 4.8 shows the DTC output as a function of time, for the case $N=4$ and FCW $=3$. The output is shown for both the ideal case of a DTC with infinite resolution and for a DTC with a finite number of bits $\mathrm{N}_{\mathrm{DTC}}=3$. 
Following similar steps as in [44] and [93], we can define the quantization error sequence $\mathrm{q}_{\mathrm{e}}[\mathrm{l}]$ as the difference between corresponding edges of the real quantized and ideal output, normalized to the DTC LSB $\mathrm{T}_{\mathrm{CK}} / 2^{\mathrm{N}_{\mathrm{DTC}}}$. The index $l$ acts as edge identifier, with $l \geqslant 1$. For our DFC, the following can be derived:

$$
\mathrm{q}_{\mathrm{e}}[\mathrm{l}] \triangleq(-1)^{\mathrm{l}}\{\mathrm{DW}[\mathrm{l}]-\mathrm{DW} \text { ideal }[\mathrm{l}]\}
$$

with

$$
\begin{aligned}
\mathrm{DW}[\mathrm{l}] & = & \mathrm{Q}\left\langle\frac{\mathrm{FCW}-\mathrm{AR}[\mathrm{l}]}{\mathrm{FCW}} \cdot 2^{\mathrm{N}_{\text {DTC }}}\right\rangle \\
\mathrm{DW}_{\text {ideal }}[\mathrm{l}] & = & \frac{\mathrm{FCW}-\mathrm{AR}[\mathrm{l}]}{\mathrm{FCW}} \cdot 2^{\mathrm{N}_{\text {DTC }}}
\end{aligned}
$$

and

$$
\begin{aligned}
\operatorname{AR}[l] & =Q\left\langle\frac{l}{2} \operatorname{GRR} \frac{\operatorname{GCD}\left(2^{N}, F C W\right)}{F C W}\right. \\
& \left.+1-2^{-\left(N_{D T C}+1\right)}\right\rangle \cdot F C W-\frac{l}{2} 2^{N}
\end{aligned}
$$

The operator $\mathrm{Q}\langle\cdot\rangle$ denotes quantization, the easiest to implement being truncation, which will be considered in this work. We will show later that the exact choice of quantization has negligible impact on spur performance. As such, since the quantization errors are smaller than one DTC LSB, $\left|\mathrm{q}_{\mathrm{e}}[\mathrm{l}]\right|<1 \forall \mathrm{l}$. Specifically, $0 \leqslant \mathrm{q}_{\mathrm{e}}[\mathrm{l}]<1$ for rising edges (odd $\mathrm{l}$ ) and $-1<q_{e}[l] \leqslant 0$ for falling edges (even $l$ ).

The periodicity of $q_{e}$ in (4.6) in number of edges $L$ is

$$
\mathrm{L}=2 \frac{\mathrm{FCW}}{\mathrm{GCD}\left(2^{\mathrm{N}}, \mathrm{FCW}\right)}
$$

Both the counter residue AR and the delay word DW (equations (4.7)-(4.9)) have period $\mathrm{L} / 2$. Moreover, the sequence $\mathrm{q}_{\mathrm{e}}[\mathrm{l}]$ has half-wave symmetry, that is $\mathrm{q}_{\mathrm{e}}[\mathrm{l}+\mathrm{L} / 2]=-\mathrm{q}_{\mathrm{e}}[\mathrm{l}]$.

We can identify the signal $s(t)$ in green in Fig. 4.8, defined in the time interval GRR $\cdot T_{C K}$, and we can express the output, that we will name $x(t)$, as a periodic extension of $s(t)$ :

$$
x(t)=\sum_{\alpha=-\infty}^{+\infty} s\left(t-\alpha G R R T_{C K}\right)
$$

Thus, the output spectrum can be written as

$$
X(f)=\sum_{h=-\infty}^{+\infty} X_{h} \delta\left(f-h \frac{f_{C K}}{G R R}\right)
$$

The output spectrum is thus a Dirac comb with impulses placed at multiples of $f_{C K} / G R R$ and complex weights $X_{h}$. Indices $h$ that are integer 


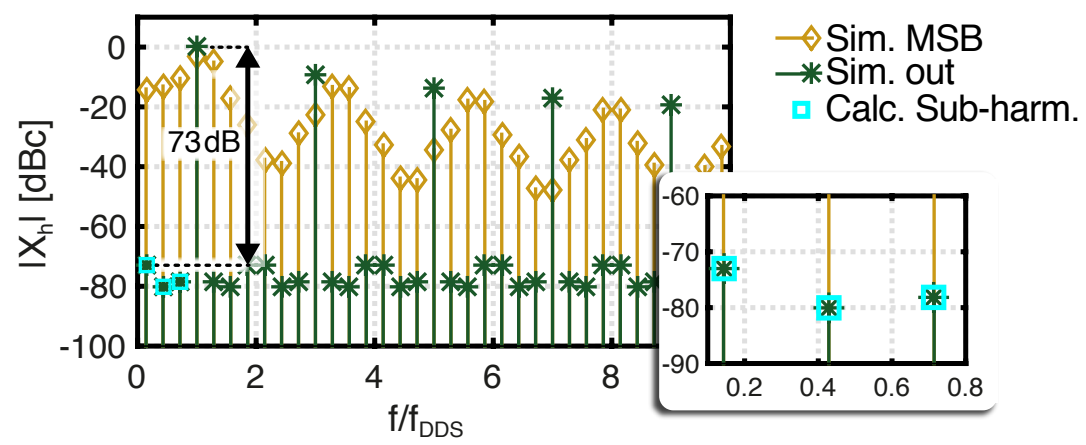

Figure 4.9: Output spectrum from behavioral simulations of the proposed $\mathrm{DFC}$, for $\mathrm{N}=12, \mathrm{~N}_{\mathrm{DTC}}=11, \mathrm{FCW}=1792$.

multiples of L/2 correspond to signal harmonics, as expressed in (4.5) for the case $h=L / 2$. The other values of $h$ correspond to fractional spurs, due to the DTC quantization error. Using the Fourier transform properties for periodic signals [103], the strength of the $h^{\text {th }}$ fractional spur can be shown to be (see Appendix B.1):

$$
X_{h} \approx-\frac{A}{G R R 2^{N_{\text {DTC }}}} e^{j 2 \pi \frac{h}{L}} Q_{e}[h]
$$

Where $A$ is the output signal amplitude, with

$$
Q_{e}[h]=\sum_{l=1}^{L} q_{e}[l] e^{-j 2 \pi \frac{h}{L} l}
$$

As the sequence $q_{e}[l]$ is real, its DFT $Q_{e}[h]$ is conjugate symmetric [104] $\left(Q_{e}[h]=Q_{e}^{*}[L-h]\right)$, meaning that the spurs repeat around each harmonic with equal absolute power. Therefore, only the sub-harmonics below the fundamental are relevant to study the output spectrum and to determine the SFDR. Due to the half-wave symmetry of the sequence $q_{e}[l], X_{h}=0$ for even values of $h$.

Fig. 4.9 shows the simulated power spectrum of both the counter's MSB and the output, as well as the calculated sub-harmonics using (4.13) and (4.14). They are plotted as a function of the normalized frequency $\mathrm{f} / \mathrm{f}_{\text {DDS }}$, for a typical example case $\mathrm{N}=12, \mathrm{~N}_{\text {DTC }}=11, \mathrm{FCW}=1792$. The calculations with (4.13) and (4.14) track the simulated spurs with an accuracy within $0.02 \mathrm{~dB}$. Clearly, DTC retiming makes a big difference: it pushes the sub-harmonic spurs down by almost $70 \mathrm{~dB}$ to $73 \mathrm{~dB}$ SFDR. The spurs are repeated around every odd harmonic. In this example, the sub-harmonics are only limited by the finite resolution of the 11-bit DTC and they are placed at odd multiples of the normalized counter rate $\left(f_{C K} / G R R\right) / f_{D D S}=1 / 7$. 


\subsubsection{Worst-case spur: closed-form estimate}

While (4.13) gives an estimate for each sub-harmonic, a closed-form estimate for the worst-case spur amplitude, given the values of $\mathrm{N}, \mathrm{N}_{\text {DTC }}$ and FCW, would be useful. It can provide a quick indication of SFDR for design purposes, e.g. to derive DTC requirements.

Note that the quantization error sequence $\mathrm{q}_{\mathrm{e}}[\mathrm{l}]$ as defined in (4.6) has a fixed offset $0.5 \cdot(-1)^{l+1}$ compared to rounding, due to the fact that DW is a truncated version of $D W_{\text {ideal }}$. Any offset in $\mathrm{q}_{\mathrm{e}}[\mathrm{l}]$ can be seen as a rigid shift of the quantized output in Fig. 4.8, it affects only the power at the fundamental frequency, without changing the output fractional subharmonics. Therefore, we will consider for the calculations the zero-offset quantization sequence:

$$
\mathrm{q}_{\mathrm{e} 0}[\mathrm{l}] \triangleq \mathrm{q}_{\mathrm{e}}[\mathrm{l}]-\frac{1}{2}(-1)^{\mathrm{l}+1}
$$

Note: $\left|\mathrm{q}_{\mathrm{e} 0}[\mathrm{l}]\right|<1 / 2 \forall \mathrm{l}$ and $\mathrm{q}_{\mathrm{e} 0}[\mathrm{l}+\mathrm{L} / 2]=-\mathrm{q}_{\mathrm{e} 0}[\mathrm{l}]$ (half-wave symmetry). As the same $\mathrm{q}_{\mathrm{e} 0}[\mathrm{l}]$ can be obtained if we assume $\mathrm{Q}\langle\cdot\rangle$ to be rounding instead of truncation, the following SFDR equations are valid for any reasonable $\mathrm{Q}\langle\cdot\rangle$-function: floor $\lfloor\cdot\rfloor$, ceil $\lceil\cdot\rceil$ or nearest integer function $\lceil\cdot\rfloor$.

Using (4.13), (4.14) and the half-wave symmetry of $\mathrm{q}_{\mathrm{e} 0}[\mathrm{l}]$, the worst-case spur relative to the fundamental output is

$$
\max _{h} \frac{\left|X_{h}\right|}{A / \pi} \approx \frac{2 \pi}{\text { GRR 2 } 2^{N_{D T C}}} \max _{h}\left|\sum_{l=1}^{L / 2} q_{\mathrm{e} 0}[l] e^{-j 2 \pi \frac{h}{L} l}\right|
$$

With $h \in[1, \ldots, L / 2-1]$. While the sign of $q_{e}[l]$ is determined by the parity of the index $l$, the sign of $\mathrm{q}_{\mathrm{e} 0}[\mathrm{l}]$ for each $l$ can be positive of negative. Thus, in analogy with data converters [73], $\mathrm{q}_{\mathrm{e} 0}[\mathrm{l}]$ can be considered a uniform random variable $\tilde{\mathrm{q}}_{\mathrm{e} 0}$ from $-1 / 2$ to $1 / 2$ and approximated in (4.16) with the average value $E\left\{\left|\tilde{q}_{\mathrm{e} e}\right|\right\}=1 / 4$. Therefore:

$$
\begin{aligned}
\max _{h} \mid \sum_{l=1}^{\mathrm{L} / 2} \mathrm{q}_{\mathrm{e} 0}[\mathrm{l}] \mathrm{e}^{-\mathrm{j} 2 \pi \frac{\mathrm{h}}{\mathrm{L}} \mathrm{l} \mid} & \approx \mathrm{E}\left\{\left|\tilde{\mathrm{q}}_{\mathrm{e} 0}\right|\right\} \max _{\mathrm{h}}\left|\sum_{l=1}^{\mathrm{L} / 2} e^{-\mathrm{j} 2 \pi \frac{\mathrm{h}}{\mathrm{L}} \mathrm{l}}\right| \\
& \approx \frac{1}{4} \frac{\mathrm{L}}{\pi}
\end{aligned}
$$

Where the last step in (4.17) comes from the following relation that is proven mathematically in Appendix B.2:

$$
\max _{h}\left|\sum_{l=1}^{L / 2} e^{-j 2 \pi \frac{h}{L} l}\right| \approx \frac{L}{\pi}
$$

Therefore, the worst-case spur can be approximated as:

$$
\max _{h} \frac{\left|X_{h}\right|}{A / \pi} \approx \frac{2 \pi}{\text { GRR } 2^{N_{\text {DTC }}}} \frac{L}{4 \pi}=\frac{1}{2^{\mathrm{N}} 2^{N_{\text {DTC }}}} \mathrm{FCW}
$$




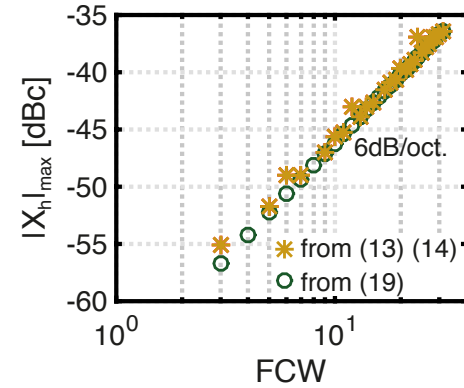

(a)

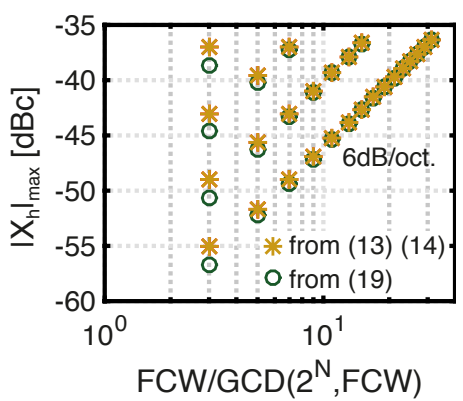

(b)

Figure 4.10: Magnitude of the worst-case spur from (4.13), (4.14) and (4.19), vs (a) FCW and (b) FCW/GCD $\left(2^{N}, F C W\right)$. Case $\mathrm{N}=6$ and $\mathrm{N}_{\text {DTC }}=5$.

Fig. 4.10a shows the magnitude of the worst-case spur (in $\mathrm{dBc}$ ) as a function of FCW, from (4.13) and (4.14), as well as its approximation (4.19). We chose the case $\mathrm{N}=6$ and $\mathrm{N}_{\mathrm{DTC}}=5$ for plot readability. The worst quantization spur does not depend on the clock frequency itself, but only on the ratio $f_{\mathrm{DDS}} / \mathrm{f}_{\mathrm{CK}}$, as can be shown by re-writing (4.19) using (4.1):

$$
\max _{h} \frac{\left|X_{h}\right|}{A / \pi} \approx \frac{1}{2^{N_{\text {DTC }}}} \frac{f_{\mathrm{DDS}}}{f_{\mathrm{CK}}} \quad(\mathrm{DFC})
$$

Moreover, from (4.19), the worst quantization spur increases by $6 \mathrm{~dB}$ every octave increase in FCW. This result can intuitively be understood by realizing that the average frequency is defined by the adder, while the DTC compensates for deterministic jitter, leaving a rather random residual quantization rms-error with power $\Delta^{2} / 12$ related to the LSB-delay $\Delta$ of the DTC. As this quantization error is roughly constant in terms of rms-jitter, its phase noise contribution scales with $1 / \mathrm{f}_{\mathrm{DDS}}^{2}$, i.e. with $6 \mathrm{~dB}$ per octave of FCW.

The error with the fitting line (4.19) depends only on L, i.e. on the ratio $F C W / G C D\left(2^{N}, F C W\right)$, as shown in Fig. 4.10b. The maximum error is $1.65 \mathrm{~dB}$ at $\mathrm{FCW}=3$ and its multiples with a power of 2 (corresponding to the same $\mathrm{L}$ ). This error is mainly due to the approximation of $\mathrm{q}_{\mathrm{e} 0}[\mathrm{l}]$ as random variable, that is less accurate for small L. Moreover, $0.4 \mathrm{~dB}$ of the total error is due to the approximation (4.18). Plots with different values of $\mathrm{N}$ and $\mathrm{N}_{\text {DTC }}$ showed that the absolute value of the spurs changes in accordance with (4.19) but the error with the fitting line stays the same and only depends on $\mathrm{L}$.

\subsubsection{Spectrum of DFCs versus DPCs}

A DFC can be compared to a DPC, such as the one shown in Fig. 4.1a, and analyzed in [44], in terms of their maximum quantization spurs, using the same DTC parameters. 


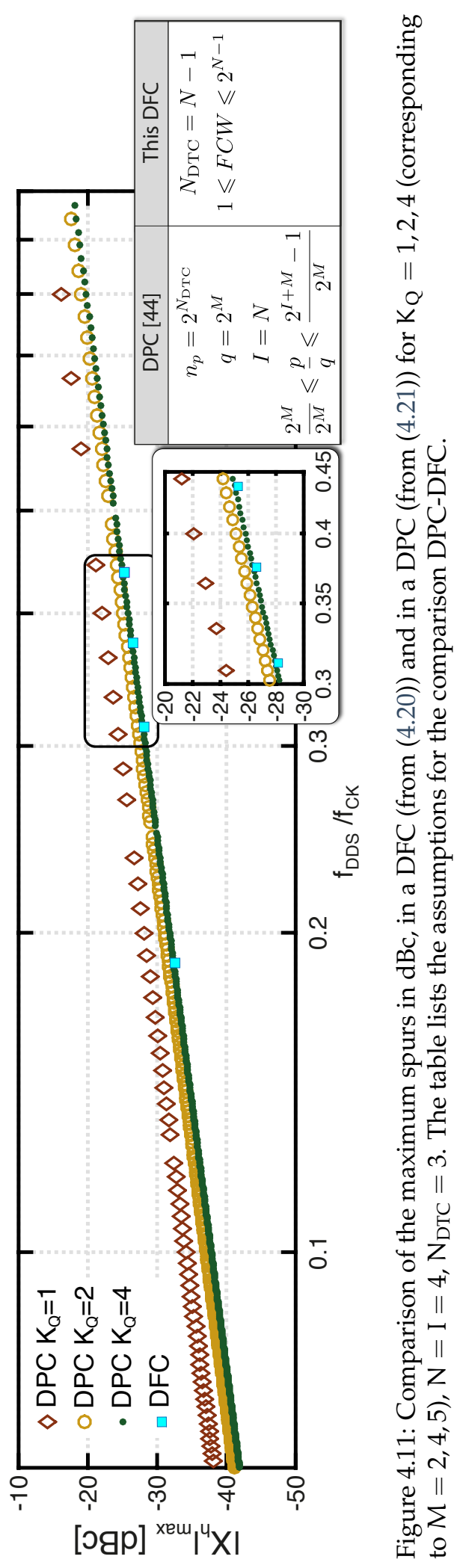

51

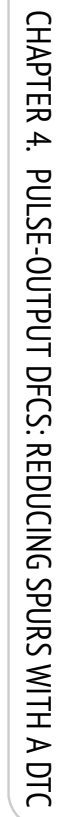


The input word of the DPC is expressed in [44] as a binary ratio $p / q$. Table 4.1 lists the variables related to the DPC in [44] and their correspondent ones related to the DFC in this work. The ratio $p / q$ is represented using I integer bits and $M$ fractional bits. The denominator $q$ is crucial to determine the periodicity of the quantization error $2 \mathrm{~K}_{\mathrm{Q}}$. It can be found by locating the right-most nonzero bit in the fractional part of the input word. By approximating the exponential in equation (46) of [44], it can be shown that for a DPC

$$
\max _{h} \frac{\left|X_{h}\right|}{A / \pi}=\left\{\begin{array}{rll}
\frac{\pi}{2} \frac{1}{n_{p}} \frac{f_{D D S}}{f_{C K}} & K_{Q}=1 \\
\frac{\pi}{2 \sqrt{2}} \frac{1}{n_{p}} \frac{f_{\text {DDS }}}{f_{C K}} & K_{Q}=2 \\
\frac{\pi}{3} \frac{1}{n_{p}} \frac{f_{D D S}}{f_{C K}} & K_{Q}=3 \\
\frac{1}{n_{p}} \frac{f_{D D S}}{f_{C K}} & K_{Q}>3
\end{array} \quad\right. \text { (DPC) }
$$

Equation (4.21) becomes the same as (4.20) for $K_{Q}>3$ and $n_{p}=2^{N_{\text {DTC }}}$. In fact, for a fair comparison, we assume equal number of quantization levels of the DTC for both the systems. The comparison DPC-DFC in terms of the maximum spurs is shown in Fig. 4.11 for three values of $\mathrm{K}_{\mathrm{Q}}$ in the DPC. For clarity, we chose an example with only a few bits $N=4$ and $\mathrm{N}_{\mathrm{DTC}}=3$. Obviously, this produces high maximum spurs, but we are interested in the spur comparison rather than the spur values. The assumptions for the comparison in Fig. 4.11 are listed in the inserted table. For the DPC, the plots in Fig. 4.11 are traced by fixing $q=2^{\mathrm{M}}$, with $p$ going from $2^{M}$ to $2^{I+M}-1$, while for the DFC, FCW goes from 1 to $2^{\mathrm{N}-1}$. The assumption $\mathrm{I}=\mathrm{N}$ ensures the maximum frequency overlap between the two systems, as can be seen from the expressions of the output frequency range in Table 4.1. The points corresponding to integer ratios $\mathrm{f}_{\mathrm{CK}} / \mathrm{f}_{\mathrm{DDS}}$ are missing in Fig. 4.11, since they do not produce fractional spurs and (4.20)-(4.21) are not valid for those points.

Different observations can be made from Fig. 4.11. As for the DPC plots, by increasing $\mathrm{K}_{\mathrm{Q}}$ from 1 to 4 , the maximum spurs decrease by $20 \log _{10}(\pi / 2)=3.9 \mathrm{~dB}$ and reaches the same spur levels as the DFC for $\mathrm{K}_{\mathrm{Q}}>3$. This means that the maximum DTC quantization spur can be made the same for DFCs and DPCs with a proper choice of $\mathrm{K}_{\mathrm{Q}}$. Increasing $\mathrm{K}_{\mathrm{Q}}$ means increasing $M$ in Fig. 4.11. This explains why the points' density in the plots increases with $\mathrm{K}_{\mathrm{Q}}$, as higher $\mathrm{M}$ implies a smaller frequency resolution in the DPC (see Table 4.1).

The main conclusion is that, at comparable output frequency range and at equal DTC, the DPC is not worse than the DFC in terms of the maximum spur achievable. However, the frequency resolution is different for the two systems. For a DPC it is not fixed, depending on the output frequency $f_{D D S}$ itself, so it changes with the input word (see Table 4.1 and Fig. 4.1a), while for a DFC is constant since it depends on quantities that do not change during the system operation.

As for the output frequency range ( $x$-domain of the plots), the upper limit $1 / 2$ in a DFC compared to 1 for the DPC is a false limitation. It can be 
Table 4.1: CORRESPONDENT VARIABLES BETWEEN [44] AND THIS WORK.

\begin{tabular}{|c|c|c|}
\hline $\begin{array}{c}\text { output } \\
\text { frequency }\end{array}$ & $\frac{T_{\mathrm{DDS}}}{T_{\mathrm{CK}}} \triangleq \frac{p}{q} \rightarrow \frac{f_{\mathrm{DDS}}}{f_{\mathrm{CK}}}=\frac{q}{p}$ & $\frac{f_{\mathrm{DDS}}}{f_{\mathrm{CK}}}=\frac{F C W}{2^{N}}$ \\
\hline $\begin{array}{c}\text { num. of DTC } \\
\text { quantiz. levels }\end{array}$ & $n_{p}^{\dagger}$ & $2^{N_{\mathrm{DTC}}}$ \\
\hline $\begin{array}{l}\text { periodicity of } \\
\text { quantiz. error }\end{array}$ & $2 K_{Q}=2 \frac{q}{\mathrm{GCD}\left(n_{p}, q\right)}$ & $L=2 \frac{F C W}{\mathrm{GCD}\left(2^{N}, F C W\right)}$ \\
\hline $\begin{array}{c}\text { output } \\
\text { freq. range }\end{array}$ & $\frac{1}{2^{I}} \lesssim \frac{f_{\mathrm{DDS}}}{f_{\mathrm{CK}}} \leqslant 1$ & $\frac{1}{2^{N}} \leqslant \frac{f_{\mathrm{DDS}}}{f_{\mathrm{CK}}} \leqslant \frac{1}{2}$ \\
\hline $\begin{array}{c}\text { frequency } \\
\text { resolution }\end{array}$ & $\Delta f_{\mathrm{DDS}}=\frac{f_{\mathrm{DDS}}^{2}}{2^{M} f_{\mathrm{CK}}}$ & $\Delta f_{\mathrm{DDS}}=\frac{f_{\mathrm{CK}}}{2^{N}}$ \\
\hline
\end{tabular}

brought to 1 at the expense of higher hardware complexity, by making the counter in the DFC sensitive to both the clock edges. In a DPC the logic is usually single-edge triggered, but indeed the upper limit 1 is due to the selection of both the clock edges from a DLL [44].

In a DPC, by changing the input word $p$, with $q$ fixed, the periodicity $2 \mathrm{~K}_{\mathrm{Q}}$ of the quantization sequence is fixed, and therefore so are the number of subharmonics. In other words, for a fixed $q$, the set of subharmonics is always the same, while $p$ changes only their position in the spectrum. In a $\mathrm{DFC}$, the periodicity $\mathrm{L}$ of the quantization sequence is proportional to the input word FCW, so the set of subharmonics cannot be reduced to a few classes. This shows there is a fundamental difference in output spectrum between DFCs and DPCs.

\subsubsection{Effect of DTC impairments on the DFC spectrum}

Any DTC impairment results in timing errors in the correction of deterministic jitter, thus causing a spur increase in the output spectrum. The DTC also introduces random jitter, but this is less of a concern, since the DTC acts on the edges of a divided signal (the pulse-output DFC can be seen as a frequency divider). The random jitter produced in recent DTC implementations is in the order of $\sim 100 \mathrm{fs}[50,61,92]$, so that the DTC contribution to the output random jitter for an output frequency of $1 \mathrm{GHz}$ would be only $0.01 \%$. Therefore, in this work we will focus only on deterministic jitter, which is manifested in the form of spurs.

A code-dependent error means that the DTC provides a deviation from the "ideal" delay that depends on its input word DW. However, from a modeling perspective, the effect on the resulting quantization error is the same as in the case of an ideal DTC with the error concentrated in DW itself. Therefore, the term DW $[l]$ in (4.6) can be expanded to incorporate the sources of errors coming from the DTC. 


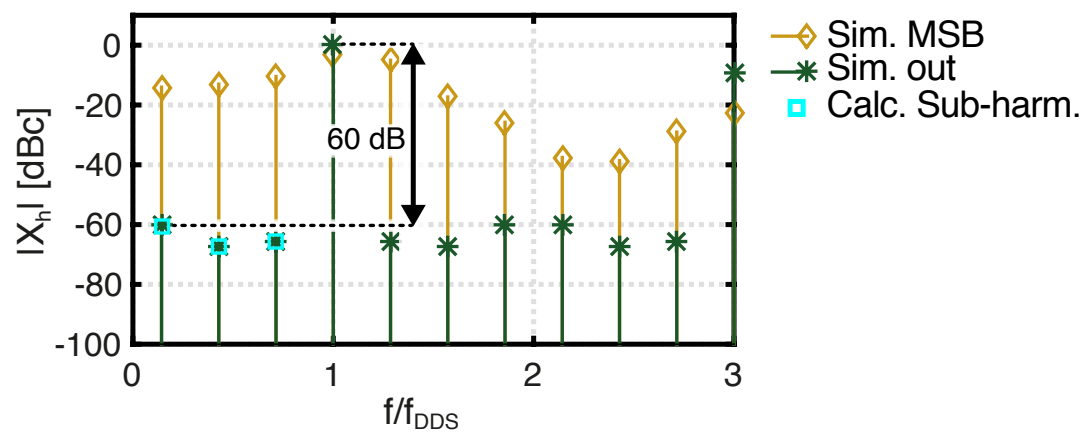

Figure 4.12: Output spectrum with DTC impairments. Parabolic INL with $\mathrm{INL}_{\max }=3 \mathrm{LSB}, 2 \mathrm{DTC} \mathrm{LSB}$ error between clock phases $\mathrm{CK}_{1}$ and $\mathrm{CK}_{2}$, and 3 DTC LSB error in the DTC fine full-scale. Horizontal axis normalized to the target output frequency. DFC with $\mathrm{N}=12, \mathrm{~N}_{\text {DTC }}=11, \mathrm{FCW}=1792$.

The DTC structure shown in Fig. 4.7 has four main causes of deterministic timing errors that affect the output spectrum: 1) INL, 2) time errors between the four clock phases, 3) full-scale time errors of the fine DTCs, 4) full-scale time mismatches between the two interleaved DTCs (R and F). Since these effects are mutually independent, they can be considered individually and then superimposed to derive a unified expression for the quantization error. All these sources of errors increase the output spurs and they can in principle be calibrated out.

\section{INL}

The DTC INL [73] is a dimensionless error only dependent on the input delay word: INL $[\mathrm{DW}[\mathrm{l}]] \triangleq \mathrm{INL}[\mathrm{l}]$. It can be directly added to DW in (4.6) so that:

$$
\mathrm{q}_{\mathrm{eINL}}[\mathrm{l}]=(-1)^{\mathrm{l}}\left\{\mathrm{DW}[\mathrm{l}]+\mathrm{INL}[\mathrm{l}]-\mathrm{DW}_{\text {ideal }}[\mathrm{l}]\right\}
$$

The DFT in (4.14) should be calculated with the quantization sequence in (4.22).

\section{Timing errors between the four clock phases}

Any timing error in the clock phases, e.g. resulting from duty-cycle errors in the reference signal, produce an error in the DTC coarse delay. As the total delay is the sum of coarse and fine delay, we can write:

$$
\mathrm{q}_{\mathrm{eCKph}}[\mathrm{l}]=(-1)^{\mathrm{l}}\left\{\mathrm{DW}[\mathrm{l}]+\mathrm{EW}_{\mathrm{CKph}}[\mathrm{l}]-\mathrm{DW}_{\text {ideal }}[\mathrm{l}]\right\}
$$

Where the error word $\mathrm{EW}_{\mathrm{CKph}}[\mathrm{l}]$ is the timing error of the current phase (function of the index $l$ ), normalized to the DTC resolution $T_{C K} / 2^{N_{D T C}}$. 


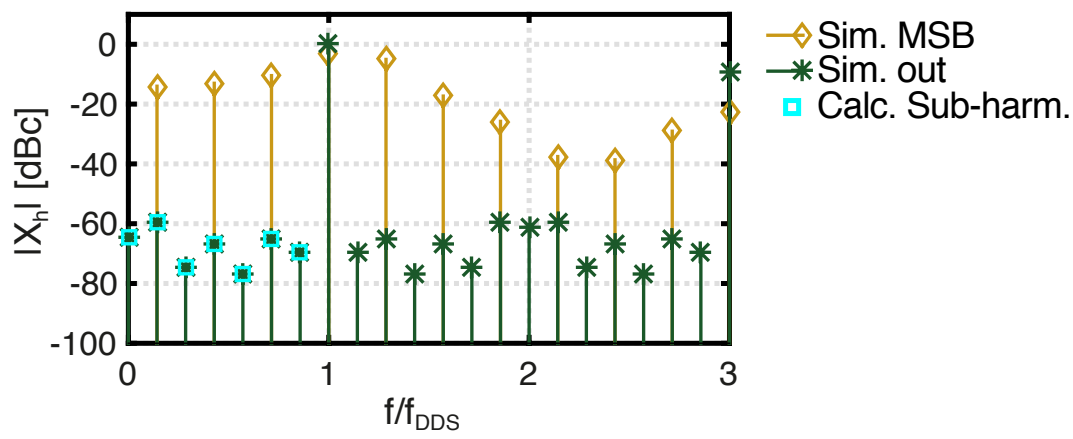

Figure 4.13: Output spectrum with DTC impairments. Same parameters as Fig. 4.12, plus 3 DTC LSB mismatch between the full-scales of the two interleaved DTCs.

\section{DTC full-scale errors}

The delay word DW can be separated into its coarse and fine components:

$$
\mathrm{DW}[\mathrm{l}]=\mathrm{DW}_{\text {coarse }}[\mathrm{l}]+\mathrm{DW}_{\text {fine }}[\mathrm{l}]
$$

For example, $1101_{2}=1100_{2}+0001_{2}$. The fine full-scale can differ from its nominal value $T_{C K} / 4$, because of variations of the period $T_{C K}$, or the DTC resolution due to PVT variations. The DTC delay (in seconds) can be written as:

$$
\tau[l]=D W_{\text {coarse }}[l] \frac{T_{C K}}{2^{N_{D T C}}}+D W_{\text {fine }}[l] \frac{\tau_{\text {FS fine }}}{2^{N_{\text {DTC }}-2}}
$$

Where $\tau_{\mathrm{FS}}$ fine is the fine delay full-scale. By applying the definition of the quantization error $[44,93]$ it is:

$$
\mathrm{q}_{\mathrm{eFS}}[\mathrm{l}]=(-1)^{\mathrm{l}}\left\{\mathrm{DW} \text { coarse }[\mathrm{l}]+\mathrm{DW}_{\text {fine }}[\mathrm{l}] \frac{\tau_{\mathrm{FS} \text { fine }}}{\mathrm{T}_{\mathrm{CK}} / 4}-\mathrm{D} W_{\text {ideal }}[\mathrm{l}]\right\}
$$

\section{Full-scale mismatches DTCs (R and F)}

If there is a mismatch between the two interleaved DTCs R and F, the fine full-scale delay $\tau_{\mathrm{FS} \text { fine }}$ in (4.26) changes depending on the DTC involved, thus becoming a function of the index $l$. Therefore (4.26) still applies, with $\tau_{\mathrm{FS} \text { fine }}$ replaced with $\tau_{\mathrm{FS} \text { fine }}[\mathrm{l}]$. This latter variable corresponds to the full scale of the DTC R or F, depending on the parity of the index $l$.

The complete equation for the quantization error when all the aforementioned sources of errors are considered is:

$$
\begin{aligned}
q_{e}[l]=(-1)^{l} & \left\{D W_{\text {coarse }}[l]+\left(D W_{\text {fine }}[l]+\operatorname{INL}[l]\right) \frac{\tau_{\mathrm{FS} \mathrm{fine}}[l]}{T_{C K} / 4}\right. \\
& \left.+E W_{C K p h}[l]-D W_{\text {ideal }}[l]\right\}
\end{aligned}
$$




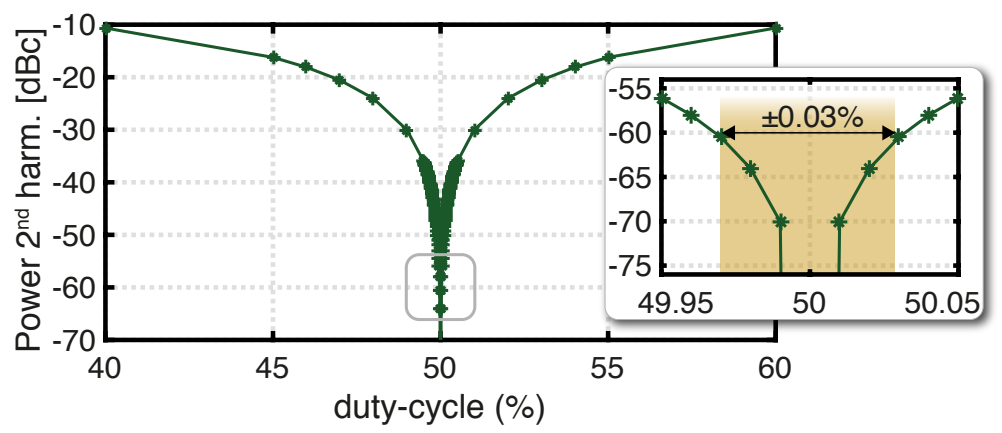

Figure 4.14: Power of the second harmonic vs duty-cycle.

As an example, Fig. 4.12 shows the spectrum in $\mathrm{dBc}$, resulting from behavioral simulations of the proposed system, when errors in the categories 1) ,2),3) have been inserted in the DTC model. In this example, we chose realistic error values of a few LSB, according to the state-of-the-art DTCs [57, 58, 92] The horizontal axis is normalized to the target output frequency. The simulated spectrum of signal MSB before the DTC correction is in yellow, the output spectrum is shown in dark green, and the calculated sub-harmonics with (4.13), (4.14) and (4.27) are shown as light-blue squares. Errors of a few LSBs in the categories 1),2),3) produce similar effects on the output spurs. They do not affect the half-wave symmetry of $q_{e}[l]$. Therefore the fractional spurs are still placed at odd multiples of $\left(f_{C K} / G R R\right) / f_{D D S}$ only, but with higher strength compared to Fig. 4.9 , where only the DTC quantization was considered. The spectrum in Fig. 4.12 has $-60 \mathrm{~dB}$ SFDR for a 11 -bit DTC, which is $13 \mathrm{~dB}$ worse than Fig. 4.9. The calculated sub-harmonics agree with simulation results within $0.02 \mathrm{~dB}$. The sensitivity to errors of type 1) and 2) is the same, as the error weights in (4.22) and (4.23) are equal, while the error of type 3 ) is scaled by the nominal full-scale, as shown in (4.26).

The effect of errors of type 4 ) is different. Fig. 4.13 shows simulations with the same parameters of Fig. 4.12, plus an extra mismatch of a few DTC-LSBs between the full-scales of the two interleaved DTCs. The result is the presence of both a DC component, even signal harmonics, and extra fractional spurs at even multiples of $\left(f_{C K} / G R R\right) / f_{D D S}$. A mismatch changes the instantaneous output duty-cycle, thus breaking the half-wave symmetry of $\mathrm{q}_{\mathrm{e}}[\mathrm{l}]$ and giving rise to extra tones in the spectrum that are tracked by (4.14). The average duty-cycle determines the strength of the even harmonics. $\mathrm{A} \pm 3 \%$ deviation around $50 \%$ in the average duty-cycle keeps the second harmonic below $-60 \mathrm{dBc}$, as shown in Fig. 4.14.

\subsubsection{Worst spur in presence of INL}

While errors of type 2),3) and 4) listed above are closely related to the DTC implementation, an INL error will always be present, so it can be useful to 


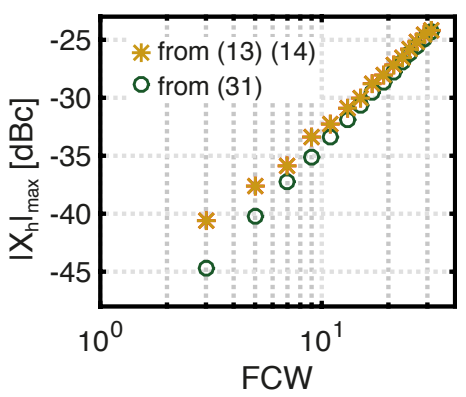

(a)

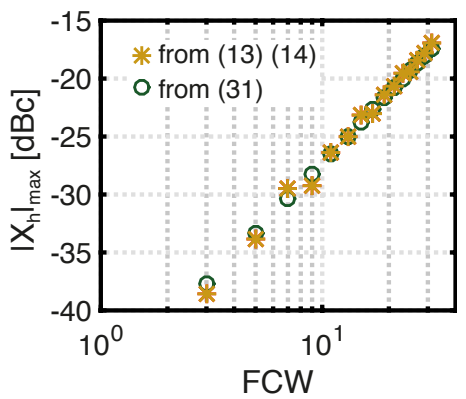

(c)

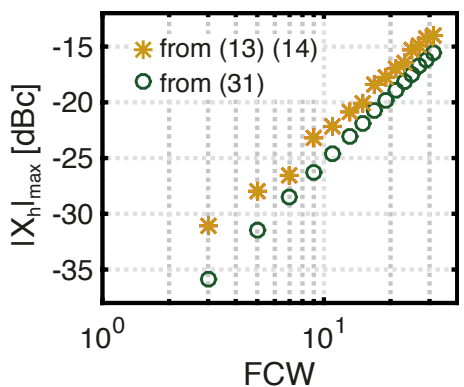

(b)

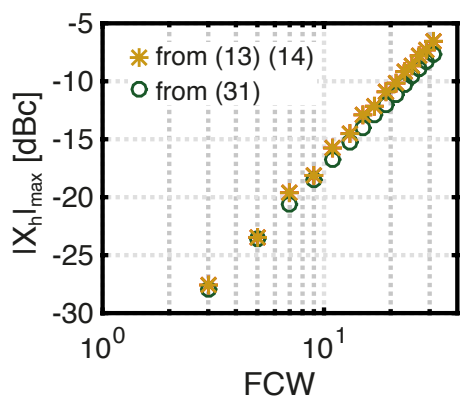

(d)

Figure 4.15: Magnitude of the worst-case spur from (4.13) and (4.14), and approximation (4.31), for $\mathrm{N}=6, \mathrm{~N}_{\mathrm{DTC}}=5$. Both plotted against odd

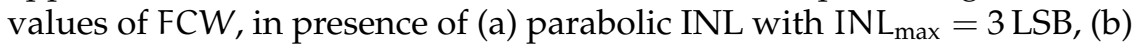
parabolic INL with $\mathrm{INL}_{\max }=10 \mathrm{LSB}$, (c) cubic INL with $\mathrm{INL}_{\max }=3 \mathrm{LSB}$, and (d) cubic INL with $\mathrm{INL}_{\max }=10 \mathrm{LSB}$.

describe the height of the maximum spur when the INL is the only DTC impairment, knowing the INL shape and maximum value $\mathrm{INL}_{\max }$.

The worst-case spur in (4.16) becomes:

$$
\begin{aligned}
\max _{h} \frac{\left|X_{h}\right|}{A / \pi} \approx \frac{2 \pi}{\text { GRR 2 } 2^{N_{\text {DTC }}}} \max _{h} \mid \sum_{l=1}^{\mathrm{L} / 2}\left\{\mathrm{q}_{\mathrm{e} 0}[\mathrm{l}]\right. \\
\left.+(-1)^{\mathrm{l}} \operatorname{INL}[\mathrm{l}]\right\} e^{-\mathrm{j} 2 \pi \frac{\mathrm{h}}{\mathrm{L}} \mathrm{l}} \mid
\end{aligned}
$$

Following the same reasoning of section 4.4.2, INL [l] can be considered a random variable IN $L$ and approximated with the expected value $E\{|I \tilde{N} L|\}$. Actually INL is a function of DW, and the sequence DW $[l]$ is dependent of the choice of the input word FCW. Therefore, for a generic FCW, we can consider DW a uniform random variable between 0 and $2^{\mathrm{N}_{\text {DTC }}-2}$ (the number of levels of the fine DTC, that has $\left(\mathrm{N}_{\mathrm{DTC}}-2\right)$ bits), and we can calculate the expected value as detailed in Appendix B.3. 
Thus, the right-hand side of (4.28) can be approximated as

$$
\begin{aligned}
E\left\{\left|\tilde{\mathrm{q}}_{\mathrm{e} 0}\right|\right\} \max _{\mathrm{h}}\left|\sum_{l=1}^{\mathrm{L} / 2} e^{-j 2 \pi \frac{\mathrm{h}}{\mathrm{L}} \mathrm{l}}\right| \\
+\mathrm{E}\{|\mathrm{IN} \mathrm{N} L|\} \max _{\mathrm{h}}\left|\sum_{l=1}^{\mathrm{L} / 2}(-1)^{l} e^{-j 2 \pi \frac{h}{\mathrm{~L}} l}\right|
\end{aligned}
$$

Where $E\{|I N \tilde{L}|\}$ depends on the shape of INL. Detailed steps for its calculation can be found in Appendix B.3, where the main results for parabolic and cubic INL shapes are:

$$
E\{|I N L[l]|\}= \begin{cases}\frac{I N L_{\max }}{4} & \text { parabolic } \\ \frac{I N L_{\max }}{2(3-\sqrt{5})} & \text { cubic }\end{cases}
$$

Therefore, combining (4.28)-(4.30) with (4.18), it is

$$
\max _{h} \frac{\left|X_{h}\right|}{A / \pi} \approx \frac{1}{2^{N} 2^{N_{\text {DTC }}}} \text { FCW }\left(1+\zeta \mathrm{INL}_{\max }\right)
$$

With $\zeta=1$ for a parabolic INL shape and $\zeta=2 /(3-\sqrt{5}) \approx 2.6$ for a cubic INL shape.

Fig. 4.15 compares the maximum spur (in $\mathrm{dBc}$ ) resulting from the analytical expressions (4.13) and (4.14) with the approximation (4.31), for both INL shapes, parabolic and cubic, with $\mathrm{INL}_{\max }=3 \mathrm{LSB}$ and $\mathrm{INL}_{\max }=10 \mathrm{LSB}$. The same number of bits as Fig. 4.10, $\mathrm{N}=6$ and $\mathrm{N}_{\text {DTC }}=5$ has been chosen, for comparison purposes. The plots show the maximum spur as function of odd FCW, so that $\operatorname{GCD}\left(2^{\mathrm{N}}, \mathrm{FCW}\right)=1$. In Fig. $4.15 \mathrm{a}$, the maximum error is $4 \mathrm{~dB}$ at $\mathrm{FCW}=3$, which decreases to $0.2 \mathrm{~dB}$ at the maximum input word $\mathrm{FCW}=31$. Similar errors have been observed for different values of $\mathrm{N}$ and $\mathrm{N}_{\text {DTC. }}$. The approximation (4.31) is less accurate for a stronger non-linearity as shown in Fig. 4.15b. In this case, the maximum error between simulation and fitting is $4.72 \mathrm{~dB}$ at $F C W=3$, and $1.62 \mathrm{~dB}$ at FCW $=31$. In Fig. $4.15 \mathrm{c}$, the maximum error is $1.1 \mathrm{~dB}$ at $\mathrm{FCW}=9$, while in Fig. $4.15 \mathrm{~d}$, the maximum error is $1.1 \mathrm{~dB}$ at $\mathrm{FCW}=31$.

Summarizing, (4.31) provides a simple equation for a crude estimate of the worst-case spur, while (4.13) and (4.14) can be used if a more accurate estimate is needed. The increase of $6 \mathrm{~dB} /$ octave of the worst-case spur with FCW is still valid in the presence of INL. The approximation of the maximum spur in (4.29) can be applied for every INL pattern, the shape factor being determined by $E\{|I \tilde{N} L|\}$, that can be calculated as shown in Appendix B.3.

\subsection{Conclusion}

In this chapter we proposed an architecture of a Pulse-Output DFC generating square waves, with a deterministic DTC-based correction scheme 
to push down the spurs in the output spectrum. We discussed the design features of a DTC suitable for this application, and developed a behavioral model of the system to find the main bottlenecks that limit the output SFDR. The results depend exclusively on the ratio between the output frequency and the clock frequency and on the DTC features (number of bits, INL and other impairments). Simulations indicate that $60 \mathrm{~dB}$ clean spectrum is possible (Fig. 4.13), with a 12-bit counter, 11-bit DTC and realistic timing errors of a few LSBs added inside the DTC model, and without resorting to orthogonal techniques for further spur reduction. Compared to existing DFC systems, that rely on dithering or $\Delta \Sigma$ techniques, the advantage of the proposed Pulse-Output DFC in terms of maximum spur goes from $5 \mathrm{~dB}$ [28] to $12 \mathrm{~dB}$ [42]. The spur performance is comparable or better also in comparison to DPC architectures, while it features linear frequency control.

The quantization error of the DTC directly affects the spur amplitude. An approximation for the worst-case spur can be determined for the case of an ideal DTC limited only by quantization noise, given the number of bits of the DTC and the frequency input word. The DTC INL can also be taken into account, knowing the INL shape and the maximum INL value, leading to a simple design equation that allows to derive DTC INL requirements given a SFDR target. The maximum spur strength, in $\mathrm{dBc}$, increases with the frequency control word by $6 \mathrm{~dB}$ per octave. 



\section{CHAPTER}

\section{Integrated DFC Prototype: UTDUCK}

In this chapter, we discuss an integrated prototype of a Pulse-Output Digital-to-Frequency Converter (DFC) with spur-correction based on a high-speed Digital-to-Time Converter (DTC). The acronym used for the prototype is UTDUCK (University of Twente Digital-outpUt ClocK ). It is a "Big-A" design [105], i. e. mixed-signal design with a strong analog focus, implemented in $65 \mathrm{~nm}$ CMOS technology. The critical blocks inside the DTC core (current source and Digital-to-Analog Converter (DAC)) are custom-designed, while the digital part has been mostly realized with automated tools.

\subsection{Top-level architecture}

The general architecture of UTDUCK is shown in Fig. 5.1. It is substantially the same as Fig. 4.7, considered in the spur analysis in chapter 4 . Here, the coarse and fine-DTCs have been highlighted in color. The system inputs are the digital word FCW and the reference clock ref together with its anti-phase version ref $f_{N}$, both sine-waves with frequency $f_{\text {ref }}$. A four-phase generator extracts four clock phases $\mathrm{CK}_{1}-\mathrm{CK}_{4}$ (square-waves), all of them having frequency $f_{C K}=f_{\text {ref }} / 2$. The word FCW is sent to the counter to control the output frequency and then its output is processed in the "logic" block, according to the algorithm described in section 4.3.2 [43], to calculate the current Delay Word DW to be sent to the DTC (see equation (4.3)). The delay added by the DTC allows to reduce the deterministic jitter at the output. The DTC can be subdivided into a coarse and fine one, depending on the amount of delay each part produces.

The coarse-DTC resamples the most significant bit MSB of the counter's output with one clock phase, selected based on the value of the two mostsignificant-bits of DW. Therefore, the resulting resampled signal MR and its inverted form $M R N$ are aligned with one of the clock phases $\mathrm{CK}_{1}-\mathrm{CK}_{4}$ 


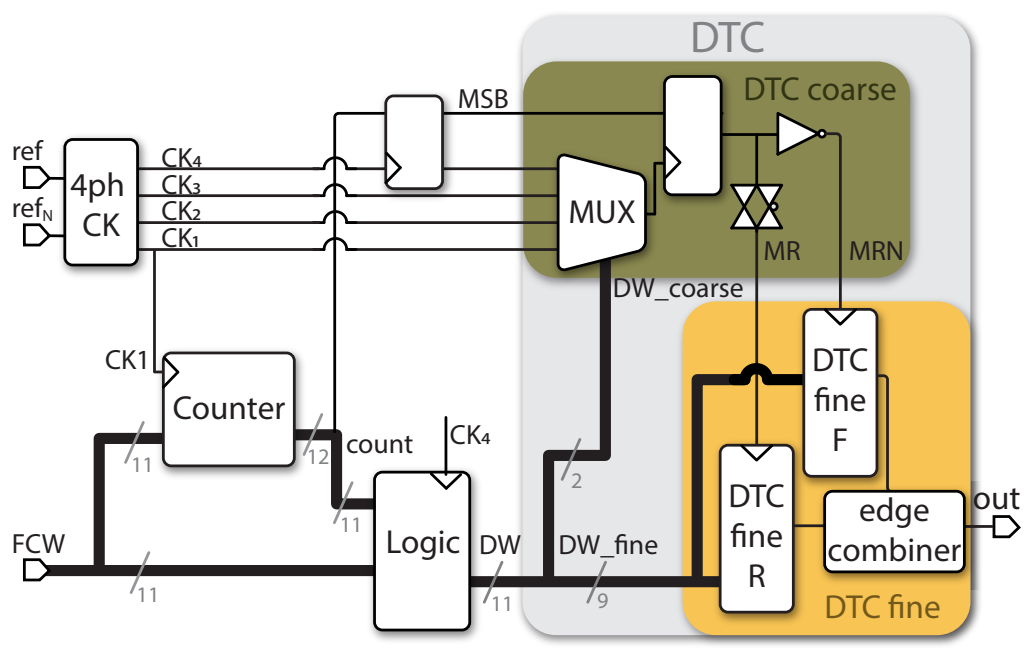

Figure 5.1: Architecture of the prototype UTDUCK, adapted from Fig. 4.7, with coarse and fine-DTCs highlighted in color.

and they are a coarse-delayed version of the counter's output MSB. Notice that the added delay is critical, since it is part of the total delay that is needed to cancel the deterministic jitter. Fig. 5.1 shows a simplified version of the coarse-DTC. The detailed implementation in the prototype will be discussed in section 5.3.2. An always-on transmission-gate has been added in the MR path (see the coarse DTC in Fig. 5.1) to compensate the inverter delay in the MRN path, in order to align the edges of the two signals MR and MRN.

After adding the coarse delay, MR and MRN are sent to the fine-DTC. It consists of two interleaved blocks, called DTC fine R and DTC fine F, each one acting on the rising and falling edge of $M R$, respectively. More precisely, the DTC-R acts on the rising edges of MR, while the DTC-F acts on the falling edges of $M R$, as shown in Fig. 5.1. Each fine-DTC, contains a DAC and a current generator, to produce the delay according to the constant slope principle discussed in chapter 2 . The two outputs from each module are then recombined (edge combiner block) to produce the system output.

\subsection{Four-phase clock generator}

The architecture of the four-phase clock generator is shown in Fig. 5.2. It is similar to the one in [81], with modified NAND gates for calibration purposes (discussed in section 5.7.2). The clock generator is based on a clock divider architecture, with a differential external clock as a reference.

The input sine-waves ref and ref $_{\mathrm{N}}$ are first converted into squarewaves $(R, R N)$ by means of two inverters with local resistive feedback (see Fig. 5.2). Sine-waves can be easily obtained in an experimental setup from 


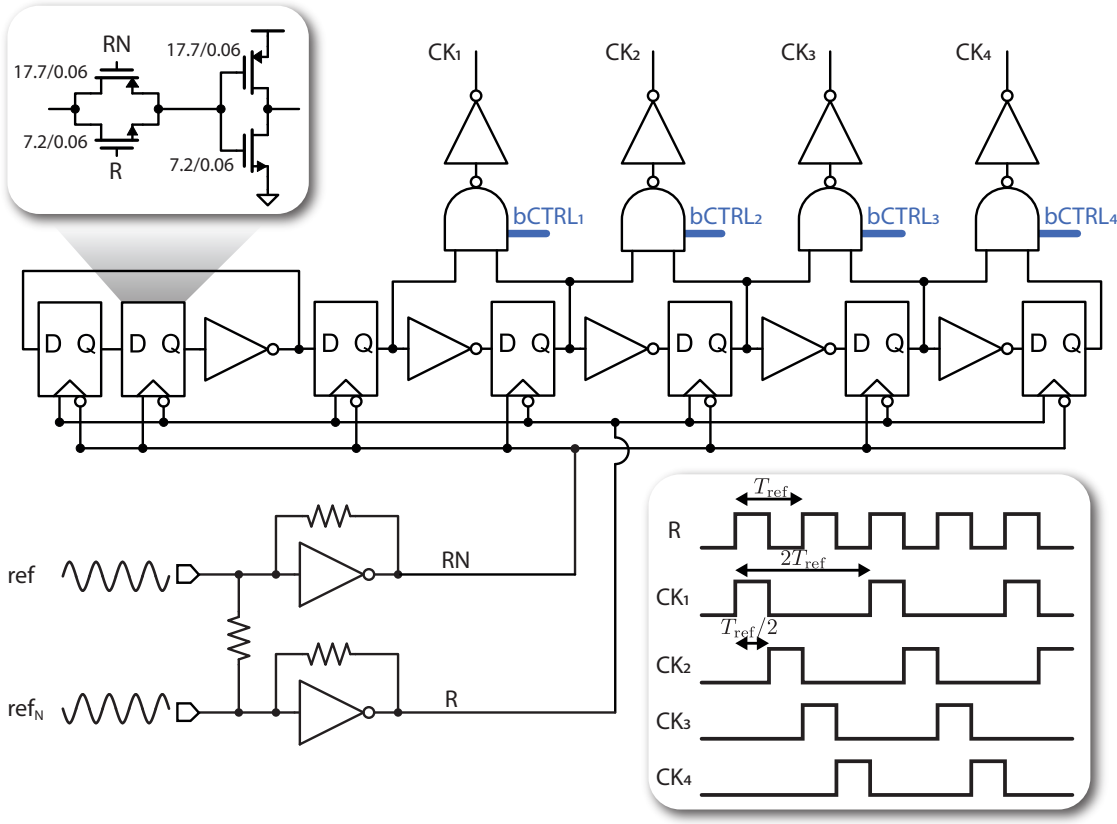

Figure 5.2: Block diagram of the four-phase clock generator, modified version of the one in [81].

a frequency generator and a balun. However the clock generator can start directly from square-waves in an integrated system. Then, the first part of the clock generation takes place in a flip-flop loop performing a frequency divide-by-two. Next, a shift register produces four delayed clocks with 50\% duty-cycle and delay $\mathrm{T}_{\text {ref }} / 2$ from each other, using both edges of signal $\mathrm{R}$. Four adjacent AND gates (implemented as NAND+inverter) combine pairs of the delayed clocks to produce $25 \%$ duty-cycled clock signals $\mathrm{CK}_{1}-\mathrm{CK}_{4}$, delayed $\mathrm{T}_{\text {ref }} / 2$ from each other. Therefore, the MSB delay (unit step of the coarse DTC) is $\mathrm{T}_{\text {ref }} / 2$. The target DTC full-scale $\tau_{F S}$ is:

$$
\tau_{\mathrm{FS}}=\mathrm{T}_{\mathrm{CK}}=2 \mathrm{~T}_{\mathrm{ref}}
$$

Where $\mathrm{T}_{\mathrm{CK}}$ is the period of each clock phase $\mathrm{CK}_{1}-\mathrm{CK}_{4}$.

\subsection{Digital-to-Time Converter}

\subsubsection{DTC Design Challenges}

The DTC design has been focused on achieving high-linearity together with an operational frequency in the $\mathrm{GHz}$ range. In fact, a linear DTC affects the cleanness of the output spectrum in a DTC-based DFC, as discussed in chapter 4, while high speed is required for high output frequency. 


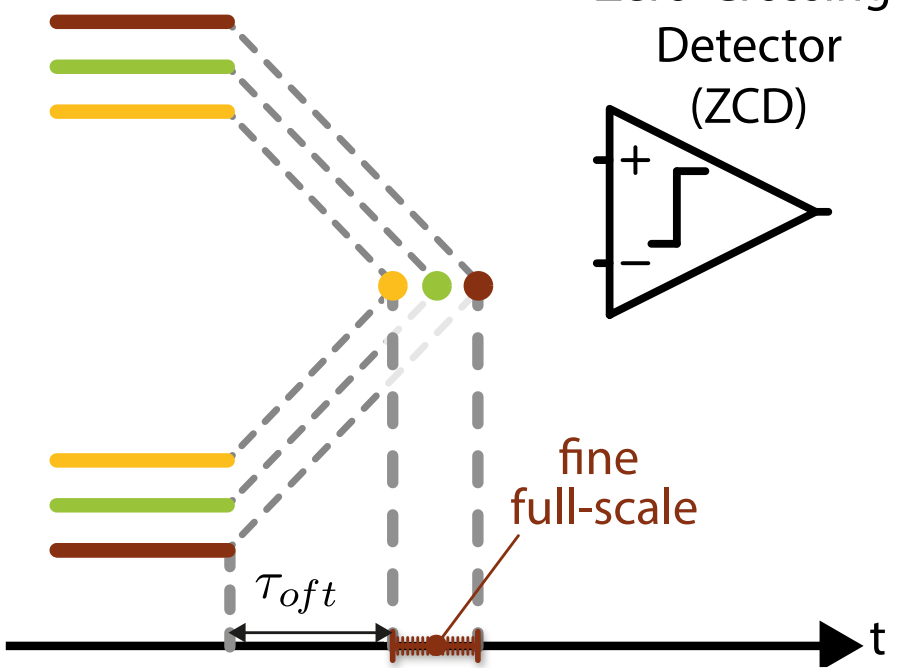

\section{Zero-Crossing}

Detector

Figure 5.3: Complementary constant-slope. Time slots adapted from Fig. 4.5b.

\section{Reducing memory-effects}

As seen in chapter 4, from a system-level point of view, the coarse-fine delay scheme and time interleaving between fine-DTCs allow a constantslope DTC to be compatible with GHz operating frequencies, addressing both the issues of DTC full-time availability and speed.

From a circuit perspective, we will now show that these two techniques are also crucial to eliminate the memory effect on the DTC capacitor (Fig. 2.4) and to improve the DTC linearity. At high frequencies, the crossing point associated with the current Delay Word (DW) can be influenced by the previous word, for example because the voltage waveforms related to the previous word have not reached the reset level, thus exhibiting memory. Since the sequence of delay words usually is not the same, this memory effect leads to DTC non-linearity, because the delay associated with the current DW would not be fully reproducible. Thus, for example, the delay-step produced with a code-step 30 (i.e. DW $=10 \rightarrow 40$ ) would not be doubled compared to the case of code-step 15 (i. e. DW $=16 \rightarrow 31$ ) because of different starting values of DW. As can be seen in Fig. 4.6, time interleaving allows to increase the time margin $\left(\tau_{\mathrm{mrg}}\right)$ for resetting the DTC capacitor, thus enabling the removal of charge residues from previous codes.

\section{Reducing disturbances affecting the crossing time}

The single-ended nature of the constant-slope circuit in Fig. 2.5 makes the threshold crossing event sensitive to any disturbance coming from 
supply and ground. This is particularly harmful at $\mathrm{GHz}$ frequencies, since the supply ripple does not have time to damp out, and in mixed-signal designs, where digital gates have a high switching activity, thus perturbing the common ground. Using a differential detection would make most of the disturbances common mode, therefore the DTC in UTDUCK is based on a differential Zero Crossing Detector (ZCD). Instead of one single ramp, the difference of two ramps is used now. This is achieved generating complementary ramps derived from complementary starting voltages. This is shown in Fig. 5.3: two starting voltages with always the same average value are initialized and then two ramps, one rising and one falling, are generated by complementary current sources. A fully-differential ZCD detects the ramps' crossing point.

Another aspect affecting the DTC linearity and the crossing instants is the colored supply noise, that is a code-dependent ripple on the power supply. This can couple to the delay path affecting the crossing time. A common way to avoid this effect is to employ a replica of the supplysensitive delay stage, in parallel to the main delay path, and drive it with a complementary code $[33,50]$. However, the power consumption of this unused dummy path makes this approach not attractive for high-speed DTC-applications. In UTDUCK, we exploit the fact that the generation of the complementary starting voltages can be made intrinsically codeindependent, when we consider the charge required to charge the two capacitors to their starting-voltages. This is because the starting voltages are differential, i.e. they have a constant common mode component and hence a constant sum, so that a constant amount of charge is withdrawn from the supply. The DAC driving scheme used in the prototype will be discussed in section 5.3.6 and proved by simulations in section 5.6.

\subsubsection{Coarse DTC}

A detailed scheme of the actual implementation of the coarse-DTC is shown in Fig. 5.4. The signal MSB (coming from the counter) is first sampled with one arbitrary phase, in this case $\mathrm{CK}_{4}$, and then sampled again with all the clock phases to produce the coarse-delayed versions MSB_1 - MSB_4. Multiplexers (MUX blocks in Fig. 5.4) select one of these signals, according to the two most significant bits of the delay word $\mathrm{DW}_{\text {coarse }}[1: 0]=\mathrm{DW}[10: 9]$, to produce MR and an auxiliary signal MRE, as well as their negative forms. Signal MRE is an early version of $M R$ that is used to initialize the starting voltages of the complementary ramps. Its role will be discussed in section 5.3.4.

By comparing the scheme in Fig. 5.1 to the coarse-DTC in Fig. 5.4, you will notice that the operations of sampling and multiplexing are swapped. Indeed Fig. 5.1 shows a simplified version of the coarse-DTC, while Fig. 5.4 corresponds to the actual implementation of it in UTDUCK. In fact, generating in parallel all the coarse-delayed signals MSB_1 - MSB_4 and 


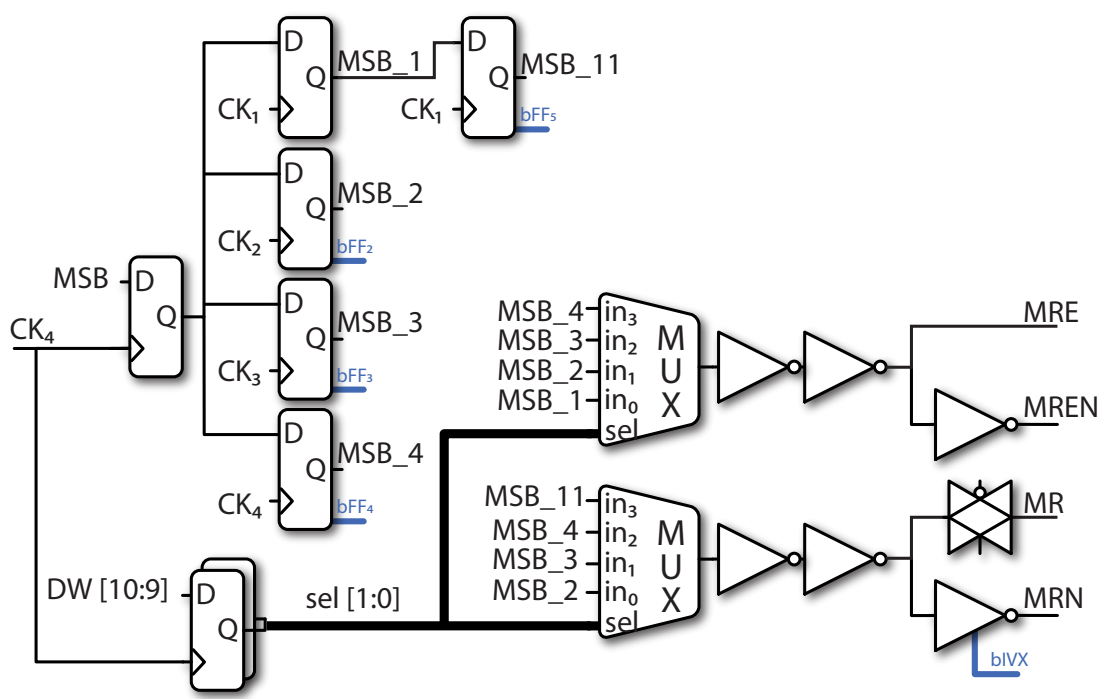

Figure 5.4: Detailed scheme of the coarse-DTC.

selecting them afterwards (as in Fig. 5.4) prevents timing violations in the resampling of signal MSB, as well as the risk of missing edges in the output $M R$, that could happen with the scheme of Fig. 5.1. A transmission gate in the $M R$ path has been inserted for delay compensation with its negative form MRN. This compensation is not necessary for the early signal MRE since its timing is not critical.

The digital gates of the coarse-DTC in Fig. 5.4 have been designed manually to better control the gate-delays, the layout of the block, and to implement accurate digital control of the edges to be used during calibration (section 5.7.2).

\subsubsection{Fine DTC: detailed view}

The implementation of the fine-DTC in UTDUCK is shown in Fig. 5.5. It mainly consists of current sources with capacitors to realize the complementary constant-slopes, two interleaved DACs and a ZCD.

The complementary ramps are produced with two current sources, $\mathrm{I}_{\text {core }}$ and $\mathrm{I}_{\text {core slave }}$ in Fig. 5.5, that charge/discharge two couples of capacitors referred to ground, being the equivalent capacitors at the nodes $A, B, C, D$. Two capacitive DACs set the starting voltages of the ramps, but their capacitors are reused as charging capacitance for the complementary ramps. Each couple of capacitors is the core of one fine-DTC: one couple for the R-path (nodes A-B in Fig. 5.5) and one couple for the F-path (nodes C-D), where $\mathrm{R}$ and $\mathrm{F}$ are names associated with the rising and falling edges of the DTC output, respectively.

While the R-couple of capacitors is developing the complementary 


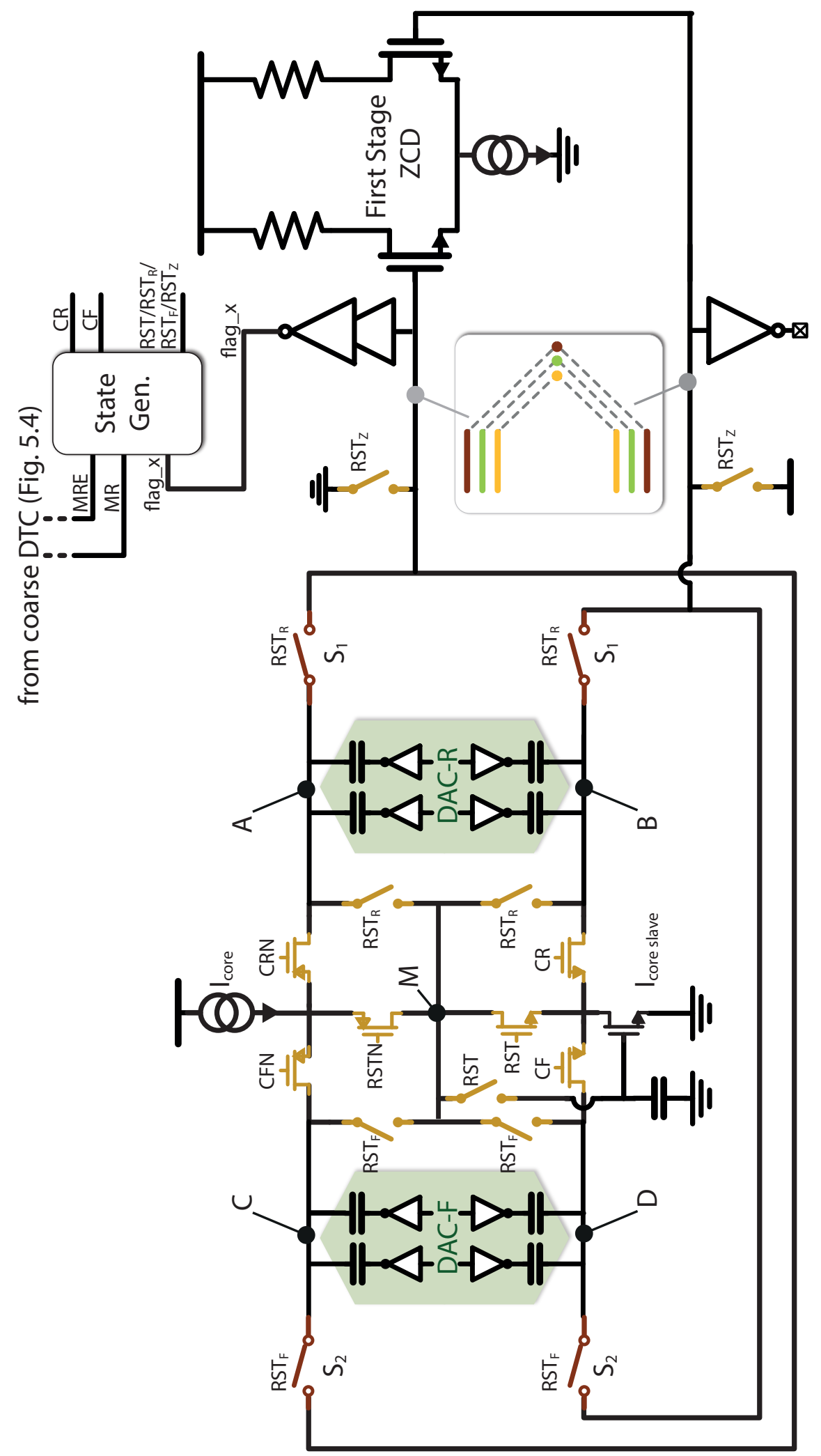


ramps, the F-couple is resetting and vice-versa. This is shown in the timing diagram in Fig. 5.7. Therefore, the current sources producing the ramps can be alternatively steered to the other couple for power efficiency. The MOSFETs used to steer the core currents are shown in Fig. 5.5. They operate in the saturation region and act as cascodes, increasing the output resistance of the current sources. They are controlled by signals produced by the state generator block. All the control signals and their role will be explained in section 5.3.4. The capacitors of each couple are reset by connecting them together and to the middle point $M$ in Fig. 5.5 through two couples of switches, controlled again by the state generator block. The multiple reset signals produced by this block have been condensed in one single line in Fig. 5.5 for clearness.

The same fully-differential ZCD is shared between the two capacitor couples, alternatively connected to each of them. In this way, all the zerocrossing detections will be made with the same offset, resulting only in a constant delay added to the output, without compromising its duty-cycle. The ZCD is connected to each DAC via two bootstrapped switches, shown in Fig. 5.5 as $S_{1}, S_{2}$ [106]. With this choice, the voltage $V_{G S}$ of the switches is kept constant, and so are their on-resistances during the ramp [74] Therefore, the inputs of the ZCD can follow the active couple of nodes $(A-B$ or $C-D)$ with the same shape, so that the linearity of the zero crossing positions is preserved.

Since switches and MOSFETs need to be switched ON/OFF in a definite sequence, with sufficient timing margin for reset to avoid memory effects, the fine-DTCs go through a precise sequence of states. The resulting state diagram is discussed in section 5.3.4.

\subsubsection{State Diagram}

In this section the state diagram of the DTC core will be presented and each state and its role will be explained. The state diagram is shown in Fig. 5.6 and it has been detailed to show the fine-DTC blocks and the switch settings for each state. The diagram is divided in two halves, depending on the level of the resampled signal MR coming from the coarse-DTC: the right half of the diagram for $M R=1$ ( $R$ path active), and the left half for $M R=0$ (F path active). We will call active capacitors the couple of capacitors currently charged, developing the complementary ramps. They alternatively belong to the $\mathrm{R}$ path and the F path (time interleaving). Notice that the signal $M R$ is both level and edge-sensitive. In fact, the level of MR determines the present state of the circuit, but $M R$ is also the first step in the generation of the output clock, with its edges being delayed by the coarse DTC. In Fig. 5.7, the main waveforms of the DTC core in Fig. 5.5 are represented over time and related to the states.

The main states of the diagram in Fig. 5.6 are the Charge for rising edge (CR) and the Charge for falling edge (CF) and they are triggered 


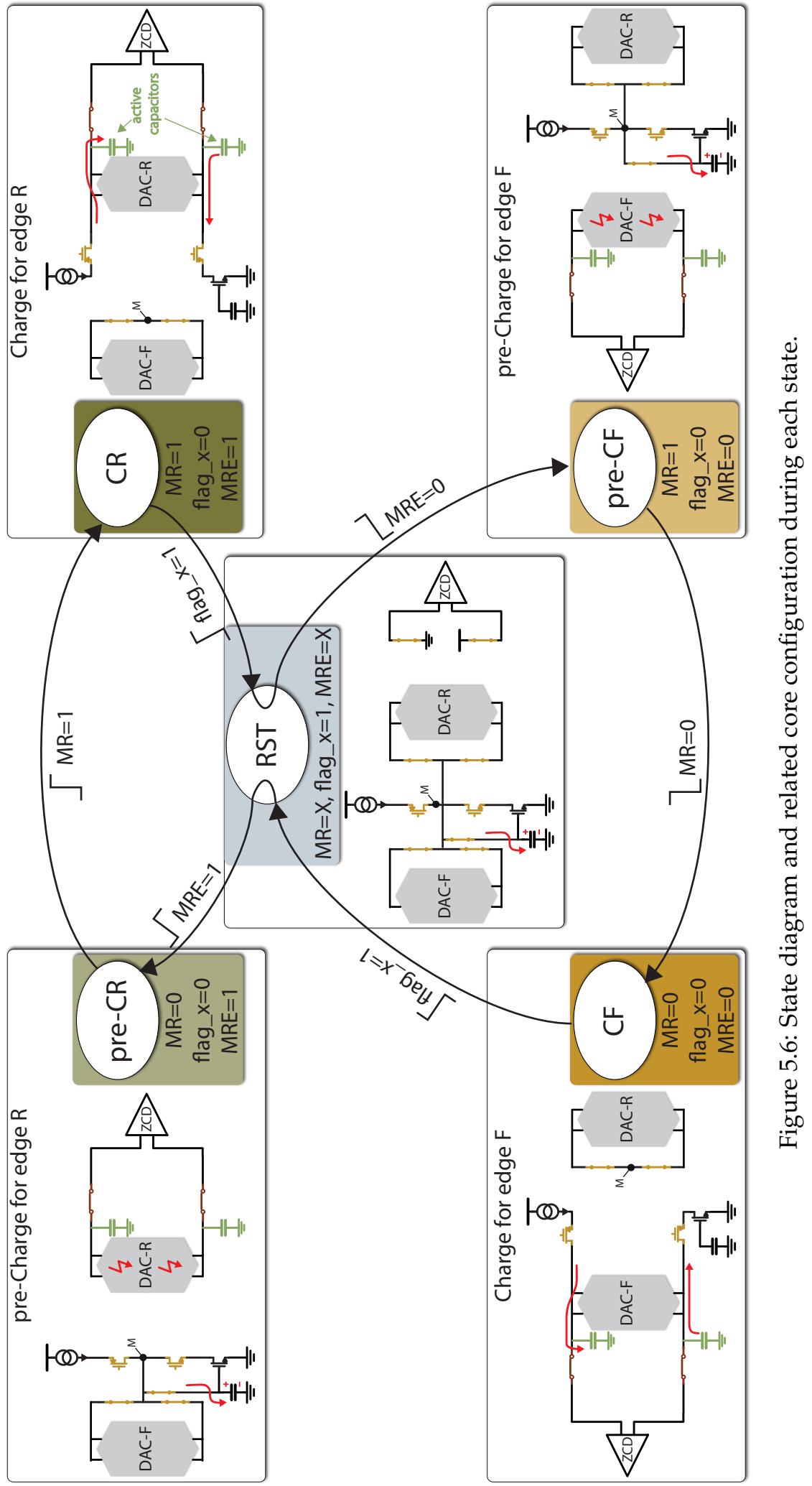

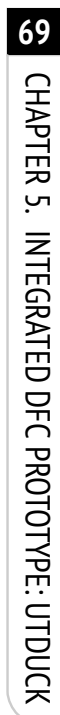


by the rising and falling edges of $M R$, respectively, as indicated on the arrows pointing to these states in Fig. 5.6. During these states, the voltage waveforms are already at their starting levels and the complementary ramps start. Only one couple of capacitors (the active fine-DTC) is involved in the complementary ramp generation, the other couple is shorted and connected to the common mode voltage (node $M$ ). The ZCD is connected to the active couple to detect the ramps' crossing. The connection is always with the same polarity, in order to provide the same offset during the detection.

The ramps' crossing triggers the Reset (RST) state, asynchronously. The variable flag_x is a flag for the crossing. Notice in Fig. 5.6 that either from the state CR ( $R$ path) or from the state CF ( F path), the value flag_ $\chi=1$ always determines the beginning of the RST state. During the RST state, all the capacitors are shorted together and one current source is diodeconnected (slave), thus copying the current of the other current source (master) [107] to enhance equality of the complementary currents. Moreover, as shown in Fig. 5.6, the ZCD is reset to a fixed full-swing unbalanced state by connecting its inputs to the supply rails, wiping out memory effects and making it ready for a new detection. The RST state begins after a certain delay from the ramps' crossing (see Fig. 5.7). This delay is one of the bottleneck of the system speed (section 5.5.1). To increase the system speed, the flag signal flag_ $x$ is used in place of the ZCD output to trigger the RST state. In principle, the RST state may seem not indispensable for the basic correct functioning of the DTC, because the time interleaved approach guarantees that the inactive couple of capacitors is shorted anyway during CR and CF states. However, RST is the only state where the ZCD is reset to the unbalanced condition (see Fig. 5.6) which is crucial for the correct DTC operation, as will be shown in section 5.5.1. Moreover, the RST state allows to periodically adjust the slave current, keeping the common mode at half the supply.

It is essential that the coming active capacitors are disconnected from the common mode voltage at node $M$ before the ramp's initial voltages (codedependent) are set, otherwise the starting levels would be affected. For this reason, the preparatory states pre-CR and pre-CF have been introduced in the state diagram (Fig. 5.6), triggered by an early version MRE of the signal MR. During these preparatory states, the couple that is going to be active (shown in green in Fig. 5.6) is disconnected from the middle node $M$ and connected to the ZCD. After this connection, the DAC conversion occurs, to set the starting levels of the ramps, as can be seen in Fig. 5.7 by comparing the voltage levels for the two cases DW $=0$ and DW $=511$.

Referring to Fig. 5.7, since both the active capacitors (nodes $A$ and $B$ ) have been reset to the same voltage (node $M$ ) during the RST state, they could trigger a false crossing event when the DAC does not change the voltage levels (case DW $=0$ ). Therefore, the couple of capacitors should be set to different voltage levels besides the DAC action. This is 


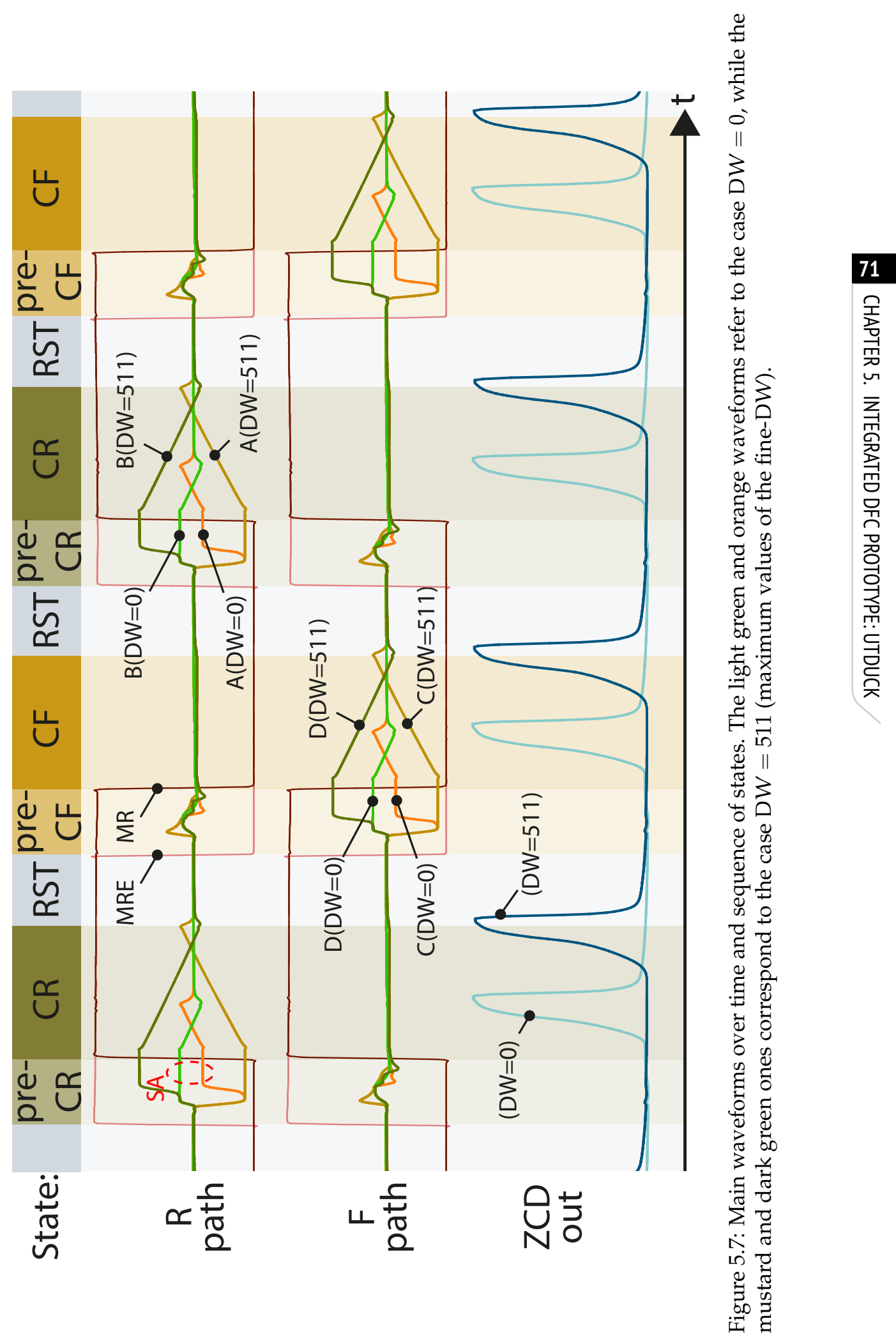


obtained by charge-sharing in a process that we will call "split-apart" (SA), highlighted with a dotted circled in Fig. 5.7. The ZCD inputs are reset to fixed full-swing during the RST state and then they are connected to the active capacitors during the pre-charge states. This produces voltage steps of opposite sign by charge-sharing, so the ramps' starting voltages split apart from each other. Therefore, even with $\mathrm{DW}=0$, nodes $A$ and $B$ start from different voltages during the states pre-CR (or pre-CF). The lack of symmetry between nodes $A$ and $B$ in Fig. 5.7 during the states Pre-CR and Pre-CF is due to the DAC driving chosen for this prototype, that will be discussed in section 5.3.6. In conclusion, the preparatory states pre-CR and pre-CF come just before the main states $\mathrm{CR}$ and $\mathrm{CF}$, and they are crucial for the proper functioning of the DTC. During these preparatory states, three main events occur: the active capacitors are connected to the $Z C D$, the SA takes place and the DAC sets a voltage increment on top of the SA voltage, depending on the current delay word.

The presence of two edges (MRE and MR in Fig. 5.7) between which the starting levels of the ramps are generated may suggest to the reader a time interpolator, as discussed in section 2.2 and section 4.2. Actually the complementary-slope method of adding delay to the output, shown in Fig. 5.7 is not a time interpolation. The early signal MRE is not combined with $M R$ to generate extra edges and, differently from $M R$, is not required to be precise. The only condition for MRE is to arrive before MR to start the necessary actions preceding the ramps start. This is substiantially different from [57] (see section 2.2 and Fig. 2.8) where the edge timing of both the clock signals $\mathrm{In}_{1}$ and $\mathrm{In}_{2}$ is essential for the correct generation of the interpolated voltages.

\subsubsection{Complementary Current Sources}

The two ramps are produced by two complementary current sources, $M_{P 1}$ and $M_{N 1}$, as shown in Fig. 5.8. The designed value for both the currents is $6 \mathrm{~mA}$ at $\mathrm{f}_{\text {ref }}=2 \mathrm{GHz}$. This choice is based on the ramp slopes requirement to meet the timing constraints: with a starting voltage that varies by $\approx$ $500 \mathrm{mV}$, a full-scale delay variation of $\mathrm{T}_{\text {ref }} / 2=250 \mathrm{ps}$ (fine full-scale) should be produced. The required slope is therefore $2 \mathrm{GV} / \mathrm{s}$. Each capacitance to be charged is $\mathrm{C}_{\mathrm{DAC}}+\mathrm{C}_{\mathrm{L}} \approx 3 \mathrm{pF}$ (table Table 5.1), thus the current required to produce the ramps is $6 \mathrm{~mA}$.

The current source in the traditional single-ended constant-slope DTC (Fig. 2.5 [CP:2] ) is suitable for $50 \mathrm{MHz}$ operational frequency, but it is not a practical solution at operation frequencies of hundreds of $\mathrm{MHz}$ or $\mathrm{GHz}$. In fact, referring to the schematic in Fig. 2.5, every time a new ramp starts, node $X$ needs first to be discharged from its reset value $V_{D D}$, to allow the pMOS current source to have enough source-drain voltage. Therefore, the desired constant current that produces the ramp needs some time to become available. Moreover, at high-speed, a new ramp could start before 


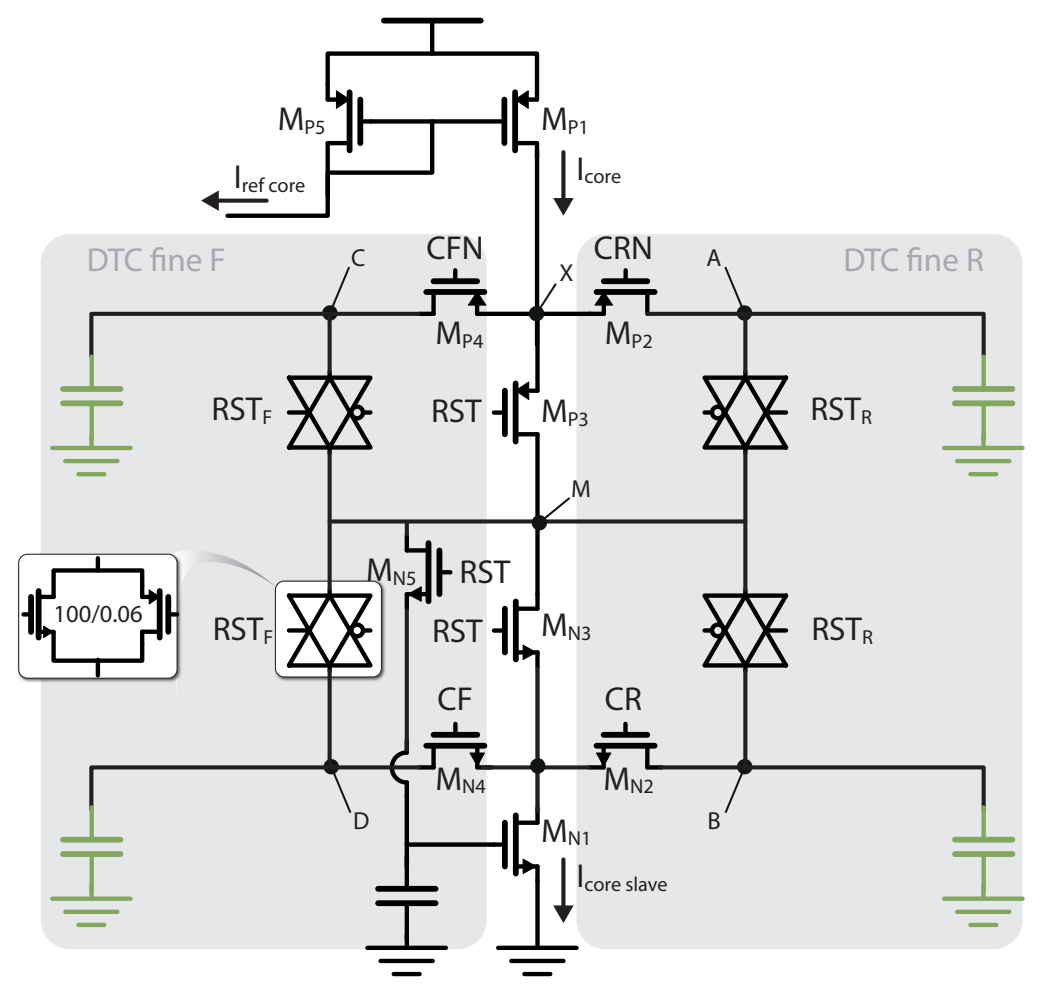

Figure 5.8: Schematic of the complementary current sources implemented in UTDUCK.

node $\mathrm{X}$ has been reset, thus causing a code-dependent memory effect in the current.

Therefore, at high-frequencies, it is convenient not switch off the current source but rather commutate in different branches, in order to have the target current immediately available to produce the ramps. This is achieved by steering the current in a different path once the charging state ends, and this approach is compatible with the time interleaving. As shown in Fig. 5.8, the steering is done with MOSFETs $M_{\mathrm{P} 2}, M_{\mathrm{P} 3}, M_{\mathrm{P} 4}$, and with $M_{N 2}, M_{N 3}, M_{N 4}$. These MOSFETs have the same size and operate in the saturation region, acting as cascodes for the current sources. Since the complementary ramps are large signals that can vary up to $600 \mathrm{mV}$ before reaching the crossing point, the cascodes should be designed to to stay in saturation during the ramps, at least until the crossing point. This will be shown with simulations in section 5.6. The minimum channel length has been chosen for the cascodes to minimize their parasitic capacitances.

The central branch of the current sources in Fig. 5.8 is a "dynamic current mirror" [107, 108]. This choice allows to match the current of $M_{P 1}$ and $M_{N 1}$. During the RST state (see section 5.3.4), the $n M O S M_{N 1}$ is periodically recalibrated by closing it in a local feedback loop. In this configuration, both $M_{N 5}$ and $M_{N 3}$ are $O N$ and the node $M$ in Fig. 5.8 has 
low impedance. Therefore, $M_{\mathrm{N} 1}$ acts as a current-mirror input transistor. It adjusts its gate voltage in order to copy the $M_{P 1}$ current. During all the other states, the switch $M_{N 5}$ is open and $M_{N 1}$ acts as current source. A MOS capacitor has been added to the gate of $M_{N 1}$ to store its gate voltage.

Switching $M_{N 3}$ off would be enough to open the loop of $M_{N 1}$ and put it in the current source configuration. So, in principle, the gate of $M_{N 1}$ could be connected to the middle node $M$. However the switch $M_{N 5}$ has been added to keep the nodes disconnected during the ramps, to avoid capacitive couplings that might produce disturbances in the nMOS ramp current.

\subsubsection{Capacitive DAC}

A capacitive DAC has been chosen in the proposed architecture. It allows for only dynamic power consumption, since the supply provides current only during the conversion. Time interleaving of the DTC requires two differential DACs, one for the rising path and another one for the falling path, that need to be initialized independently.

Referring to Fig. 5.9, the driving scheme of each differential DAC has been developed to achieve three main targets:

1. reset of both the outputs of the differential DAC to a common voltage;

2. draw a code-independent current from the DAC reference voltage during the DAC conversion;

3. generation of complementary voltages at the outputs.

The first target is needed to share the same voltage to the two outputs (nodes A and B in Fig. 5.9) during reset. In this way, disturbances on the reset voltage will become common mode. The second target addresses a possible cause of delay modulation and therefore non-linearity [33, 61]. A code-independent current is obtained with a fixed total number of transition $0 \rightarrow 1$ when going from reset to the DAC conversion. The third target is necessary to generate the complementary starting levels of the ramps, as shown in Fig. 5.3, meaning $\left(V_{A}+V_{B}\right) / 2=V_{M}$.

The starting point is the differential DAC with binary weights shown in Fig. 5.9a. For simplicity, only a 3-bit DAC has been drawn and the voltage levels will be indicated with 0 and 1 to refer to ground and the DAC reference voltage, respectively. During reset, the first target is achieved in that all the capacitors are connected to the common-mode voltage $V_{M}$ and to 0 . The second target is accomplished by using complementary codes during the DAC conversion. However, the driving scheme in Fig. 5.9a does not achieve the third target, as can be seen from the voltage waveforms in Fig. 5.9a, where $V_{B}$ is higher than $V_{A}$ instead of complimentary.

The third target of complementary levels can be achieved by doubling the capacitors and driving them asymmetrically, as shown in Fig. 5.9b. 
Reset:

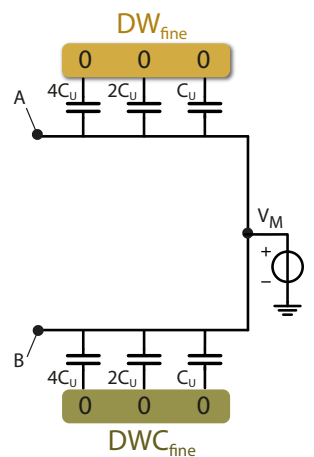

DAC conversion

(example):

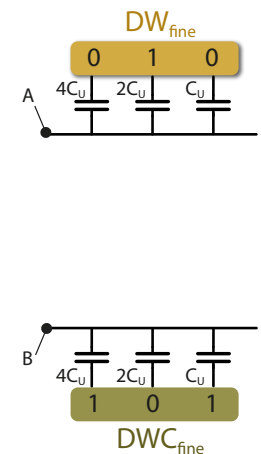

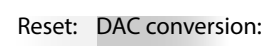

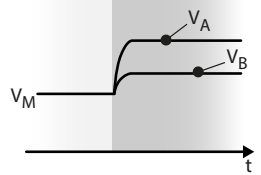

(a) Driving the DAC with complementary codes.

Reset:

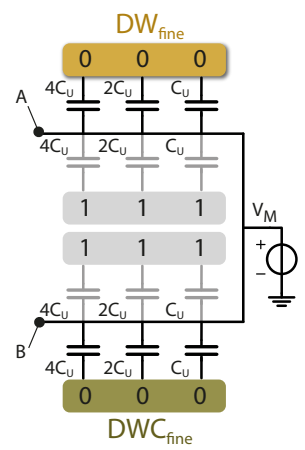

DAC conversion (example):

(b) Asymmetric DAC driving and doubled capacitance.

Reset:

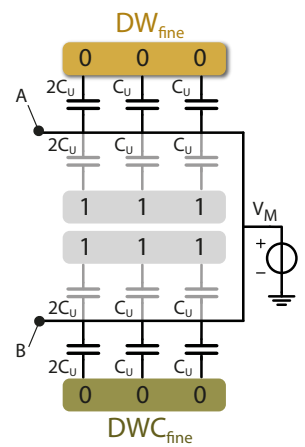

DAC conversion (example):

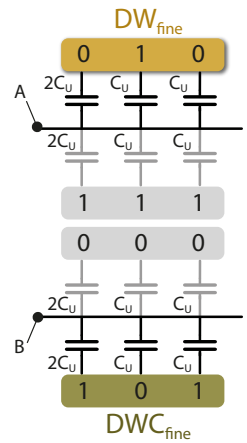

Reset: DAC conversion:

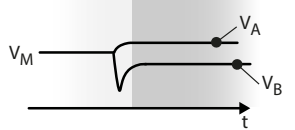

(c) Asymmetric DAC driving and reduced capacitance.

Figure 5.9: Logic steps to achieve the current DAC driving scheme. Simple example with a 3-bit DAC. Nodes A and B are the same as Fig. 5.8. With 0 and 1 we refer to ground and the DAC reference voltage, respectively. 
DAC conversion
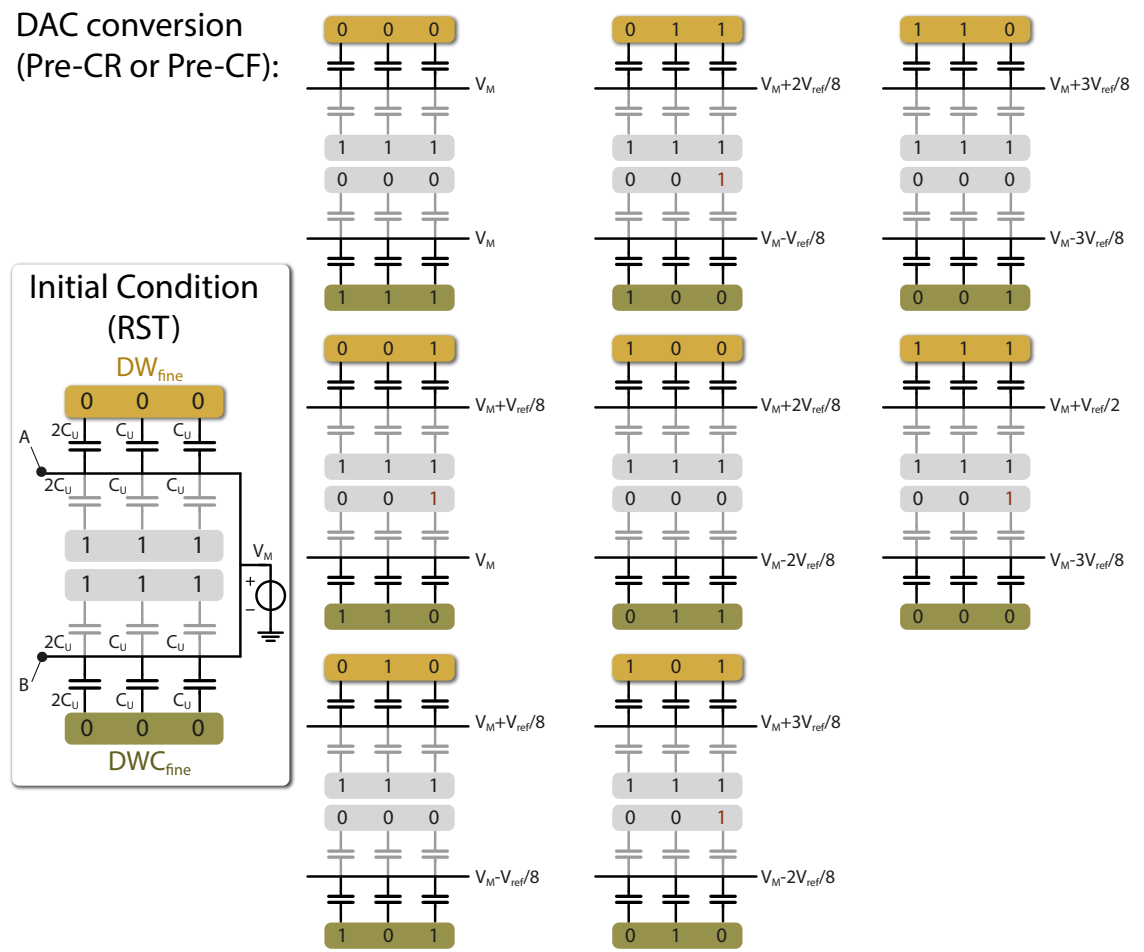

Figure 5.10: Example of DAC programming in the prototype. Simple case of a 3-bit DAC. The capacitors' weights in the circuits on the right, omitted for readability, are the same as the circuit on the left.

In particular, the added capacitors (in gray in Fig. 5.9b) are connected to 1 during reset, but the ones related to node $B$ are put to 0 during the DAC conversion. In this way, half of the capacitance connected to node $B$ produces a voltage drop that, combined with the voltage increase due to the complementary word $D W C_{\text {fine, }}$, produces a final voltage $V_{B}$ which is complementary to $\mathrm{V}_{\mathrm{A}}$.

The approach of Fig. 5.9b is not efficient in terms of area and dynamic power consumption, since the total capacitance has been doubled. Moreover, at equal code, the voltage steps has been reduced by half, as can be seen by looking at $V_{A}$ in both the time diagrams of Fig. 5.9a and Fig. 5.9b. However, the same behavior of Fig. $5.9 \mathrm{~b}$ can be obtained with the weight scheme of Fig. 5.9c, where the total capacitance differs by only two units $\mathrm{C}_{\mathrm{u}}$ compared to Fig. 5.9a. The two least significant bits have the same weight, then for each following bit the corresponding weight scales up in a binary way. The weight scheme in the proposed capacitive DAC is inspired by the SAR ADCs in [109, 110], however the driving scheme is new for this design.

Fig. 5.10 shows the driving scheme for the entire set of input words, for the case of a 3-bit DAC, chosen for simplicity. Notice that in case of an 


\section{$\mathrm{DW}_{\text {fine }}$}

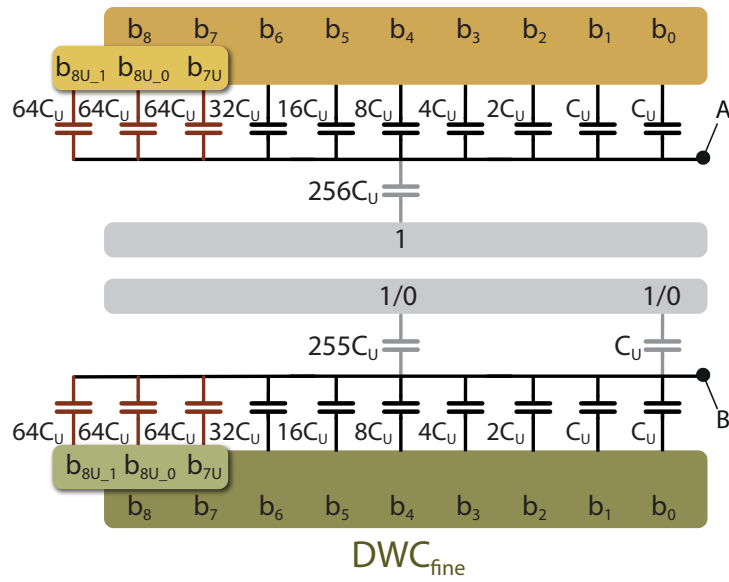

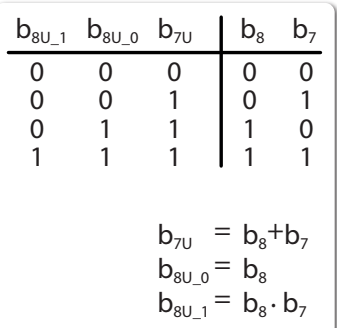

Figure 5.11: Scheme of the 9-bit capacitive DAC used in the prototype. The nine bits $b_{0}-b_{8}$ of the fine word $D W_{\text {fine }}$ are connected to the associated DAC capacitors. The weights related to $b_{0}$ and $b_{1}$ are the same, according to the proposed DAC driving scheme. The bits $b_{8}$ and $b_{7}$ are converted to unary before driving the corresponding DAC capacitors. The conversion is realized with combinational logic, according to the truth table shown on the right.

odd DW (e.g. DW = 001) the voltage at node $A$ does not change compared to the next word (e.g. DW $=010$ in Fig. 5.10). This is due to the fact that the two LSBs of DW are connected to two equally weighted capacitances. With the driving scheme of Fig. 5.9c, the same would be true for node B, resulting in the same differential voltage for both the codes $D W=001$ and $\mathrm{DW}=010$. An easy solution to get the correct differential voltages is to change the common output voltage by 1 LSB for odd values of DW, as shown in Fig. 5.10, by keeping one capacitance $C_{u}$ on node $B$ connected to 1 during the DAC conversion. This will increase the common mode of nodes $A$ and $B$ by 1 LSB and will reduce their differential voltage by 1 LSB. Therefore, the DAC differential output will differ by 1 LSB when going from an odd DW to the next value of DW. The resulting $1 \mathrm{LSB}$ common mode variation for odd DW will not affect the linearity if it is inside the common mode input voltage range of the following ZCD.

The actual differential DAC implemented in the prototype UTDUCK is 9-bit, shown in Fig. 5.11. One set of capacitances is driven with the 9-bit delay word DW $W_{\text {fine }}$ that controls the DTC fine-delay, while the other set is driven with $D W C_{\text {fine }}$ which is the ones' complement of $D W_{\text {fine }}$. A segmented architecture has been chosen, with the first two MSBs capacitors unary weighted to improve the Differential Nonlinearity (DNL). Therefore, the binary bits $b_{8}$ and $b_{7}$ in Fig. 5.11 are converted to their unary equivalent $\mathrm{b}_{8 \mathrm{u} \_} 1, \mathrm{~b}_{8 \mathrm{u}} \mathrm{u}_{0}$ and $\mathrm{b}_{7 \mathrm{u}}$, before driving the capacitors. 


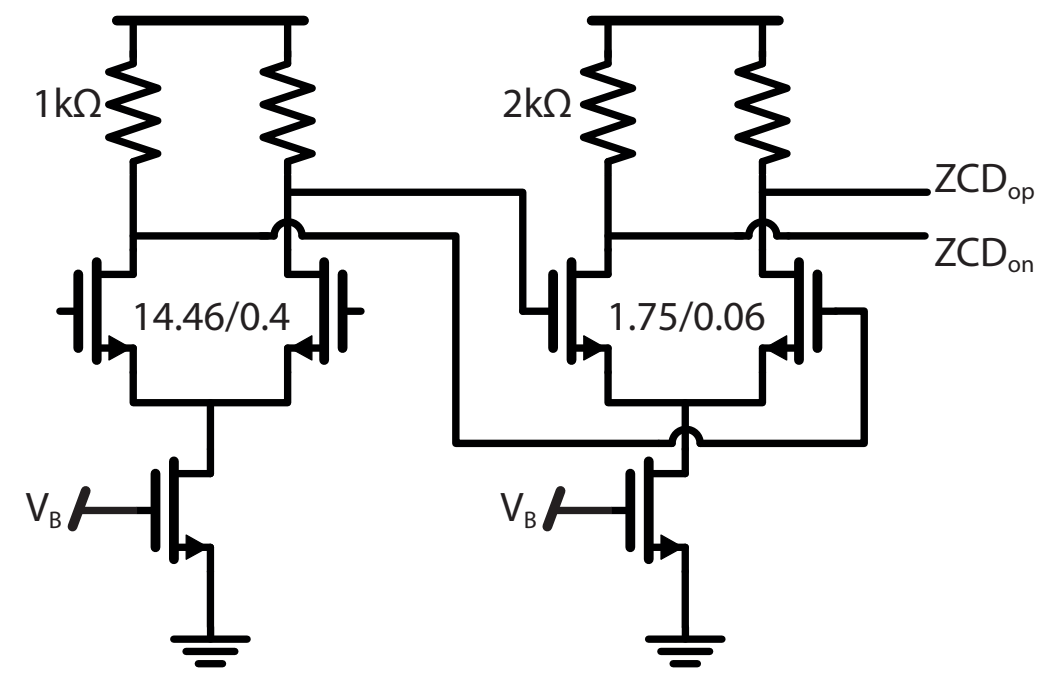

Figure 5.12: Zero Crossing Detector in the prototype.

\subsubsection{Zero Crossing Detector}

The ZCD detects the ramps' crossing and is the last block of the fineDTC. It is implemented in the prototype as a cascade of two Low-Voltage (LVT) differential pairs with resistive loads, as shown in Fig. 5.12, and it is connected to the active couple of capacitors through the bootstrapped switches $S_{1}, S_{2}$ shown in Fig. 5.5.

The ZCD inputs during the charging states have a complementary constant-slope shape like Fig. 5.3. It is important that both the MOSFETs of the first differential pair remain $\mathrm{ON}$ and in saturation during the crossing event. The MOSFETs employed for the differential couple are not minimum length to improve their flicker noise and to avoid Reverse Short-Channel Effects (RSCEs) [111] on their threshold voltage. To guarantee a fast response, a wide bandwidth is required. For this reason, a simple differential-pair topology with passive load has been chosen. The MOSFETs sizes and resistance values are shown in Fig. 5.12. Two stages have been put in cascade to increase the small-signal gain. The ZCD bandwidth is $3.5 \mathrm{GHz}$, determined by the RC constant of the second stage.

\subsection{Final stages}

After the ZCD, there are output buffers for measurement purposes, implemented with a fully-differential chain in Current-Mode Logic (CML). This choices reduces changes in the supply rails and keeps the chain to the output fully differential, thus allowing an accurate propagation of the edge to the output. The final stages of the prototype are shown in Fig. 5.13. The ZCD operates at double the frequency of the signal MR. The ZCD output, shown at the bottom row of Fig. 5.7, has its rising edges controlled 


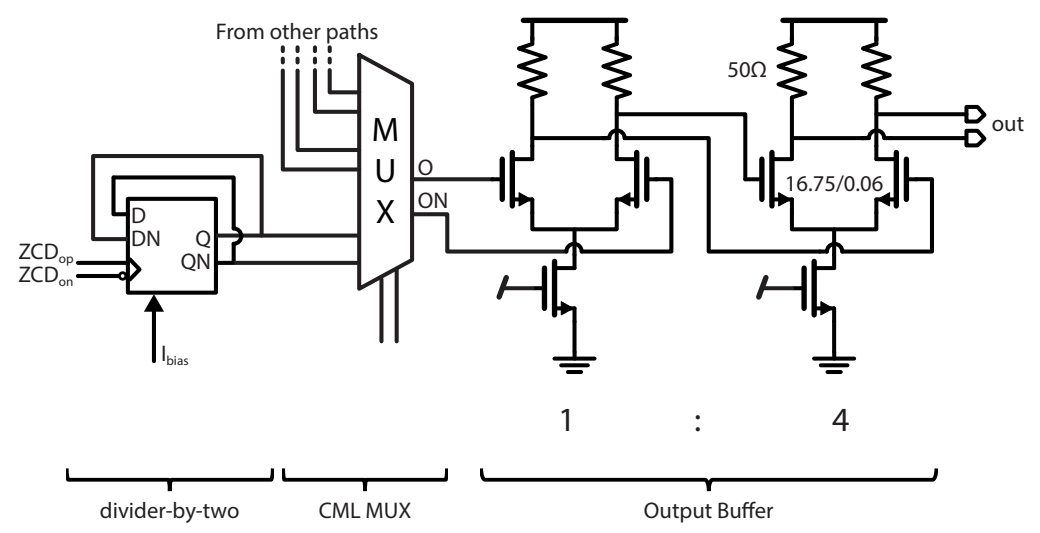

Figure 5.13: Zero Crossing Detector in the prototype.

by the digital word DW and its falling edges determined by the RST state (see the case DW = 511 in Fig. 5.7). A following flip-flop divides the frequency by two, discarding the ZCD falling edges, so that the resulting output waveform (not shown in Fig. 5.7) has both edges controlled only by DW. The divider by 2 is also realized in CML, consisting in a D-flip-flop with local feedback. After the divider, the signal passes through a $3 \times 1$ multiplexer, to eventually select other paths designed for measurement purposes (see section 5.7). Finally, a CML output buffer drive the output to the external termination. It consist of a chain of two tapered CML stages. The resistors of the last stage are on-chip $50 \Omega$ terminations.

\subsection{Expected performance and limitations}

\subsubsection{Time constraints for the time-interleaved DTC}

Now that the DTC architecture (Fig. 5.1), circuit implementation (Fig. 5.5) and state diagram (Fig. 5.6) have been clarified, it is possible to identify the time constraints and speed limitations of the system. To do so, we will identify the slower path and the critical condition for the DTC to work correctly.

Since the clock phases are all derived from an external reference (section 5.2), we will express all the fixed time intervals as function of the period $T_{\text {ref }}$ of the external reference.

Fig. 5.15 shows a time diagram of the sequence of states. The different times in the diagram are closely related to the implementation of the state generator and the fine-DTC, shown in Fig. 5.14 and Fig. 5.5, respectively. The triggering edges of each state are placed in the first two rows, while the subsequent edges that derive from them are shown in the lower lines. The edges of MR and MRE are synchronized with a clock phase and, therefore, with the external reference. Thus, they are triggering edges and they are placed in the top row of Fig. 5.15. The edges of flag_x are asynchronous, as 


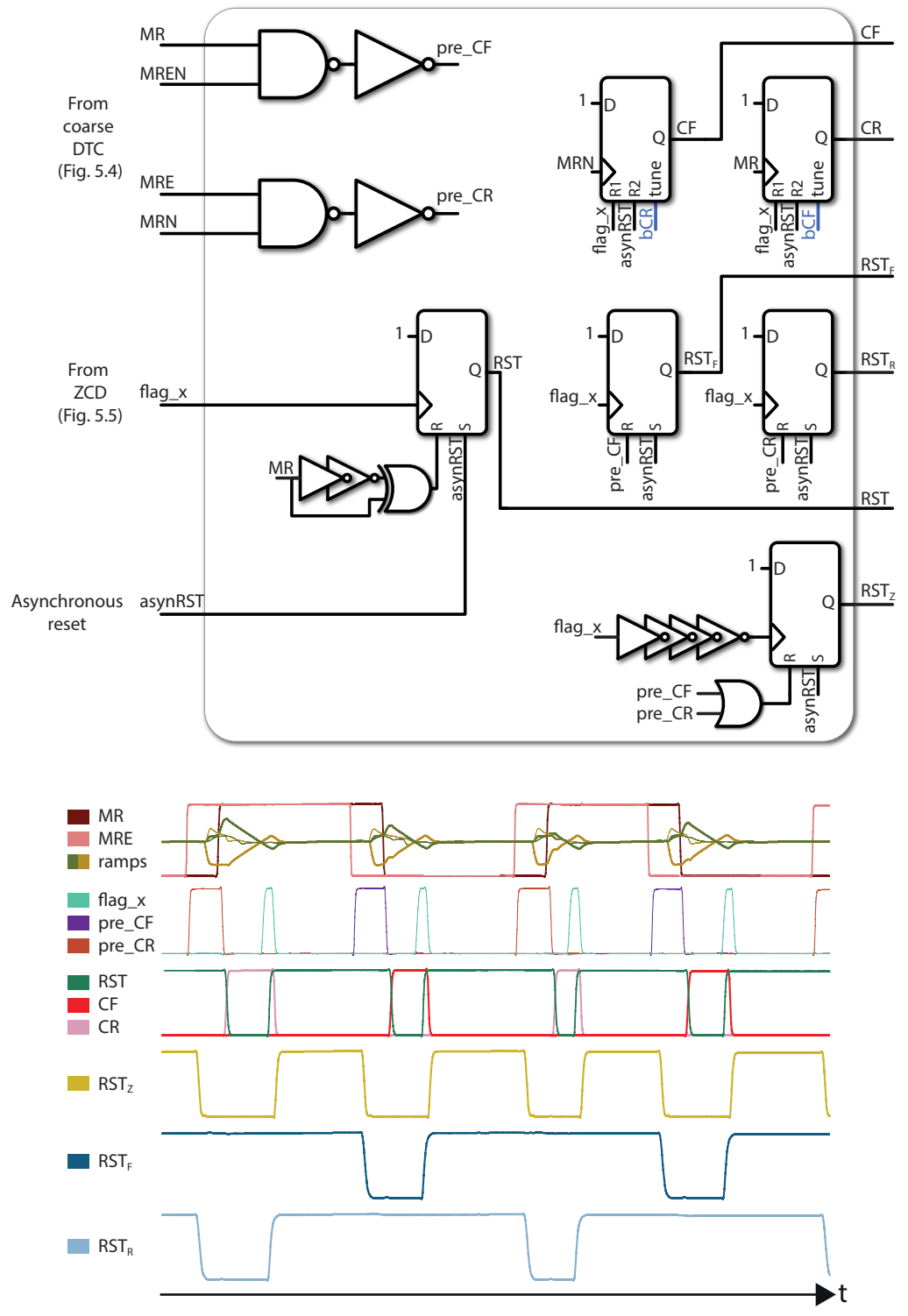

Figure 5.14: Schematic of the state generator and associated waveforms. The delay word changes at every edge of $M R$, so the ramps are all different from each others.

they are related to the crossing event, and they are placed in the second row of Fig. 5.15. Grey arrows link each master edge with the derived signals. The delay expressions between related edges are indicated next to each arrow.

The position of an MR edge, in the timing grid of the external reference, is related to the overflow of the counter (i.e. FCW) and the coarse value $D W_{\text {coarse }}$ of the delay word. Instead, the distance between an edge MR and 


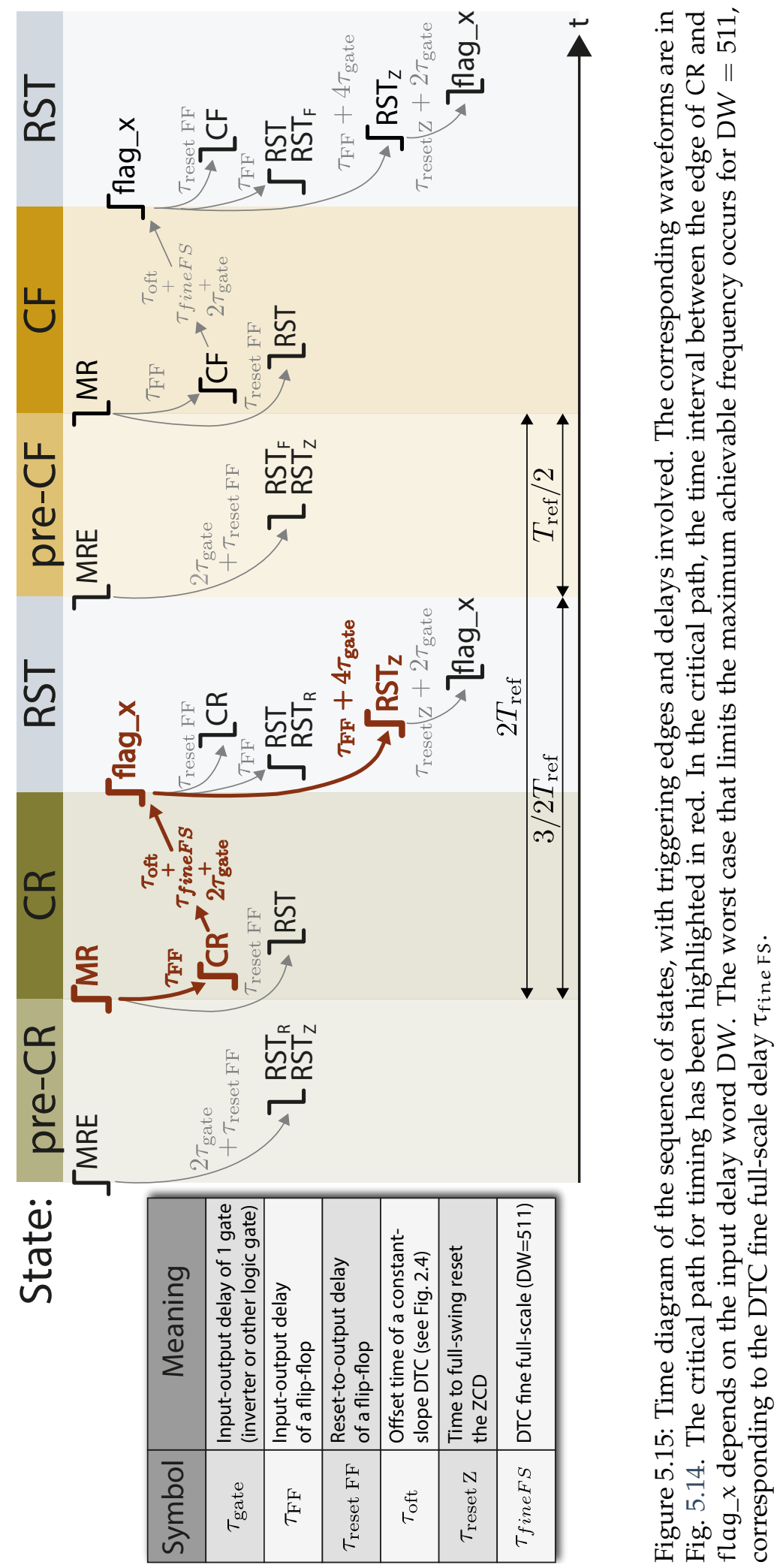

81

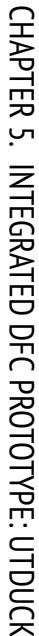


the following edge flag_ $x$ depends on the fine value $D W_{\text {fine }}$ of the delay word. The critical case for timing occurs when two consecutive MR edges are the closest possible and the fine delay to be produced is the highest possible (DTC fine full-scale delay). Fig. 5.15 refers to this critical case.

The closest spacing for two consecutive $M R$ edges is $2 \mathrm{~T}_{\text {ref }}$, i.e. the period of one clock phase (Fig. 5.2). The time difference between MRE and MR edges is fixed at $T_{\text {ref }} / 2$.

In order for the DTC to work properly, the ZCD outputs should go to the unbalanced values (reset) before a new charging state starts, to be ready for a new detection, even in the most critical condition when the DTC provides the maximum fine delay (fine full-scale $\tau_{\text {fine } \mathrm{FS}}=\mathrm{T}_{\text {ref }} / 2$ ) and $M R$ is the fastest. This means that the signal $R_{S T}$, responsible for the ZCD unbalance, should go high before the following MRE edge occurs.

$$
\mathrm{t}\left(\mathrm{RST}_{z}\ulcorner) \leqslant \mathrm{t}(\operatorname{MRE} \mathrm{Z})\right.
$$

This condition has been proven to be critical by transient simulations (see section 5.6). The critical time-path shown in green in Fig. 5.15 goes from the independent edge MR to the related edge RST $T_{Z}$. The speed condition is:

$$
\tau_{\mathrm{FF}}+\tau_{\text {oft }}+\tau_{\text {fineFs }}+2 \tau_{\text {gate }}+\tau_{\mathrm{FF}}+4 \tau_{\text {gate }} \leqslant \frac{3}{2} \mathrm{~T}_{\text {ref }}
$$

Where the meaning of the symbols is listed in Fig. 5.15. The quantities $2 \tau_{\text {gate }}$ and $4 \tau_{\text {gate }}$ in (5.3) correspond to the actual circuit implementation. For example, the signal flag_ $x$ is obtained at the output of two cascaded inverters (as shown in Fig. 5.5), therefore it is obtained after $2 \tau_{\text {gate }}$.

Equation (5.3) allows to calculate an upper limit for $f_{\text {ref }}=1 / T_{\text {ref }}$ to estimate the maximum speed of the DTC (coarse+fine). As for the DFC system, the speed bottleneck can be the DTC or the digital part. Custom design of flip-flops and inverters in the state signal generator permits to obtain flip-flop delays as low as $\tau_{\mathrm{FF}}=40 \mathrm{ps}$ and inverter delays $\tau_{\text {gate }}=$ $10 \mathrm{ps}$ in $65 \mathrm{~nm}$ TSMC. Both $\tau_{\text {oft }}$ and $\tau_{\text {fineFs }}$ are set to $T_{\text {ref }} / 2$. Therefore, from (5.3) it is

$$
f_{\text {ref }} \leqslant 3.6 \mathrm{GHz}
$$

A reference frequency of $3.6 \mathrm{GHz}$ results in a frequency for clocks $\mathrm{CK}_{1}-\mathrm{CK}_{4}$ equal to $\mathrm{f}_{\mathrm{CK}}=1.8 \mathrm{GHz}$.

In conclusion, the expected maximum DTC speed is at around $1.8 \mathrm{GHz}$, so the DTC is expected to independently delay both the edges (rising and falling) of an input signal up to $900 \mathrm{MHz}$. Thus speed limitation is due to the necessity to reset the ZCD to the unbalanced condition during the RST state (see Fig. 5.6). Since the entire DFC system also contains digital blocks in standard TSMC65 CMOS logic and these blocks can run at a few $\mathrm{GHz}$, the speed bottleneck of the entire system is given by the DTC. This expectation will be compared to the measured results in section 5.7.7. 
Notice that in (5.3), $\tau_{\text {oft }}$ is unavoidable in a constant-slope DTC and $\tau_{\text {fineFs }}$ is the required fine full-scale delay, therefore with the chosen topology, the only way to increase the system speed is to reduce the values of $\tau_{\mathrm{FF}}$ and $\tau_{\text {gate. }}$. These values are actually limited to the technology, so this design would benefit from going to finer technology nodes.

\subsubsection{Output jitter}

We will now calculate the expected output jitter of the prototype. Referring to the overall scheme in Fig. 5.1, the main jitter contributor is the fine-DTC, since it produces the ramps, i.e. analog delays, which tend to contribute jitter more than dividers [102]. The following blocks in Fig. 5.1 are digital circuits that can be considered jitterless, since the signal after the ZCD has achieved its maximum slope [112]. The jitter of one clock phase should also be added to obtain the total output jitter $\sigma_{\mathrm{t}}^{2}$. In fact the delay produced by the fine-DTC is added on top of the coarse delay, which is determined by the edge position of the selected clock phase:

$$
\sigma_{t}^{2}=\sigma_{t_{\text {fine-DTC }}}^{2}+\sigma_{t_{C K}}^{2}
$$

Where $\sigma_{t_{\text {fine-DTC }}}^{2}$ is the jitter of the fine-DTC and $\sigma_{\mathrm{t}_{\mathrm{K}}}^{2}$ is the jitter of one clock phase. It can be considered the same as the input reference jitter, since each clock phase is obtained from the input reference and a combination of digital cells, as shown in section 5.2.

The jitter of the fine-DTC can be obtained from the output voltage variance, using the relation [112]:

$$
\sigma_{t_{\text {fine-DTC }}^{2}}^{2}=\frac{\sigma_{v_{\text {out }}}^{2}}{S L_{\text {out }}^{2}}
$$

Where $\sigma_{v_{\text {out }}}^{2}$ is the total output voltage variance and $S \mathrm{~L}_{\text {out }}$ is the output slope at the zero crossings.

We will now consider the contribution of each noise source using Fig. 5.16 to calculate the output variance $\sigma_{v_{\text {out }}}^{2}$. The critical points with respect to noise are the nodes where the ramps develop ( $A$ and B in Fig. 5.16), since in these nodes the signal is the slowest of the circuit and the noise currents are integrated in the DTC capacitors for the entire duration of the ramps. The sources of noise of the DTC analog core are shown in Fig. 5.16, together with the fine-DTC configuration during the state CR. After the ZCD, the CML digital circuit towards the output can be considered jitterless, since the signal has achieved the maximum slope. From a noise perspective, the outputs we will consider for each noise contribution are the two outputs of the ZCD's second stage. Only thermal noise will be considered here.

The channel thermal noise of the pMOS current source, with Power Spectral Density (PSD) $S_{I_{C}}$, is integrated over the total capacitance at node $A$ during the ramp time $\tau_{\text {thr }}$, according to the random walk model 


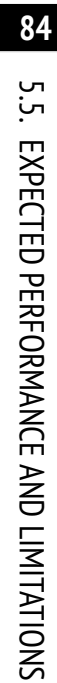

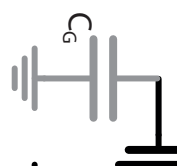

节节

룽 $\stackrel{\circ}{\stackrel{0}{5}}$

ค

苛

อิ

政

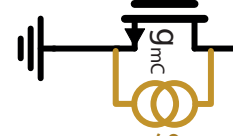

]
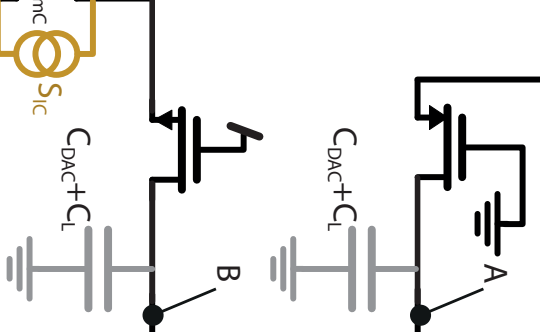

.

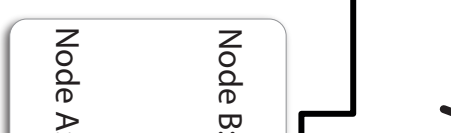

$\sum_{n}$

Б)

คั

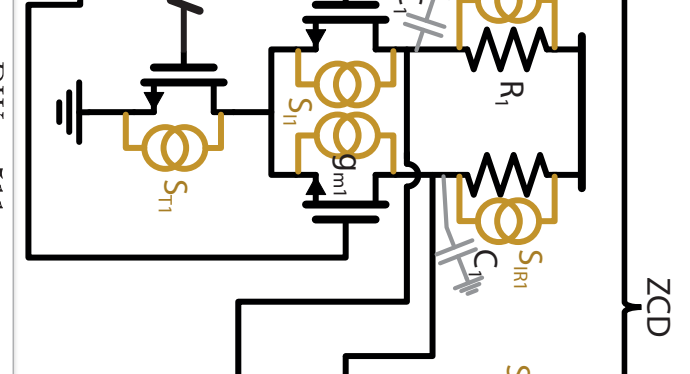

范

웅

$\frac{8}{8}$

궁
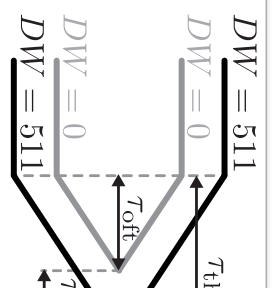

T-1

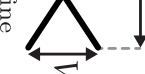




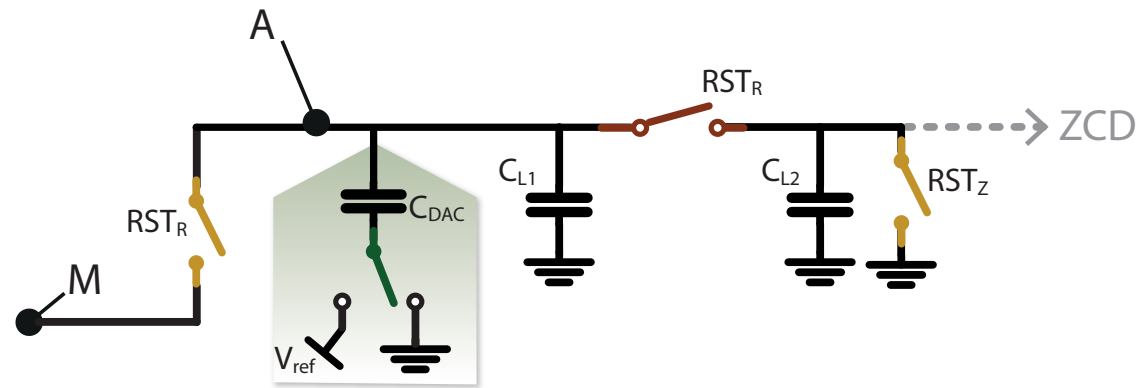

Figure 5.17: Scheme of the switches and capacitances involved for the calculation of the total $\mathrm{kT} / \mathrm{C}$ noise. Adapted from Fig. 5.5. The inverters in the DAC cells can be considered switches either connected to the DAC reference voltage or to ground. The value $C_{D A C}$ refers to the total DAC capacitance.

$[113,114]$. The same holds for the thermal noise $S_{I_{R}}$ of the reference side of the current mirror, but it is first multiplied by the squared of the mirror ratio. Their contribution to the output voltage variance is

$$
\sigma_{v_{\text {Ip }}}^{2}=\frac{S_{I_{C}}+S_{I_{R}} 100}{\left(C_{\text {DAC }}+C_{L}\right)^{2}} \tau_{\text {thr }}^{2} \frac{1}{2 \tau_{\text {thr }}} R_{1}^{2} g_{m_{1}}^{2} R_{2}^{2} g_{m_{2}}^{2}
$$

Where $C_{\text {DAC }}+C_{L}$ is the total DTC capacitance, $1 / 2 \tau_{\text {thr }}$ is the noise equivalent bandwidth and $R_{1}^{2} g_{m_{1}}^{2} R_{2}^{2} g_{m_{2}}^{2}$ is the squared differential voltage gain of the ZCD. The thermal noise of the nMOS current source is integrated at node $B$ and has the same transfer to the output as the noise at node $A$. The transconductance $g_{m_{C}}$ of the nMOS is designed to be equal to the one of the pMOS current source, so they nominally have the same PSD $S_{I_{C}}$.

The nMOS is in a dynamic current mirror configuration, therefore the noise current due to the $\mathrm{kT} / \mathrm{C}$ noise of its gate capacitance $\mathrm{C}_{\mathrm{G}}$ should also be considered. The total contribution to the output voltage variance is

$$
\sigma_{v_{I n}}^{2}=\left[\frac{S_{I_{C}}}{\left(C_{D A C}+C_{L}\right)^{2}} \frac{\tau_{\text {thr }}}{2}+\frac{k T}{C_{G}} \frac{g_{m_{C}}^{2}}{\left(C_{D A C}+C_{L}\right)^{2}} \tau_{\text {thr }}^{2}\right] R_{1}^{2} g_{m_{1}}^{2} R_{2}^{2} g_{m_{2}}^{2}
$$

The capacitances $C_{D A C}+C_{L}$ at nodes $A$ and $B$ are subject to thermal noise associated with the switches ( $\mathrm{kT} / \mathrm{C}$ noise). Fig. 5.17 shows the set of switches that play a role in the $\mathrm{kT} / \mathrm{C}$ noise at node $A$, but a similar scheme is also valid for node B. The inverters in the DAC unit cells can be seen as switches connecting the bottom plate either to the supply or ground. The load capacitance $C_{L}$ has been split into its components $C_{L_{1}}$ and $C_{L_{2}}$ in Fig. 5.17. The resulting voltage variance at the outputs of the $Z C D^{\prime}$ s second 
stage is

$$
\begin{gathered}
\sigma_{v_{C}}^{2}=2[\underbrace{\frac{k T C_{\text {DAC }}}{\left(C_{\text {DAC }}+C_{L}\right)^{2}}}_{\text {switch DAC }}+\underbrace{\frac{k T}{\left(C_{\text {DAC }}+C_{L}\right)}}_{\text {switch RST }_{R}} \\
+\underbrace{\frac{k T C_{L_{2}}}{\left(C_{\text {DAC }}+C_{L}\right)^{2}}}_{\text {switch RST }}+\underbrace{\frac{k T}{\left(C_{\text {DAC }}+C_{L}\right)}}_{\text {bootstrap }}] R_{1}^{2} g_{m_{1}}^{2} R_{2}^{2} g_{m_{2}}^{2}
\end{gathered}
$$

Where the different contributors have been labeled. The factor 2 results from considering both the active nodes $A$ and $B$. The derivation of the DAC part and of the RST $\mathrm{Z}_{\mathrm{Z}}$-switch part has been detailed in appendix $\mathrm{C}$.

As for the ZCD stages in Fig. 5.16, all their components - differential pairs, tail current generators and load resistances - contribute to noise. In the first stage, the thermal noise of the load resistors causes voltage noise at the differential output of the second ZCD stage, whose variance is

$$
\sigma_{v_{\mathrm{ZCD}_{1 \mathrm{R}}}}^{2}=2 \mathrm{~S}_{\mathrm{I}_{\mathrm{R}_{1}}} \mathrm{R}_{1}^{2} \mathrm{BW} \mathrm{W}_{\mathrm{n}} \mathrm{g}_{\mathrm{m}_{2}}^{2} \mathrm{R}_{2}^{2}
$$

Where the factor 2 is due to the independence of the noise generators associated with the two load resistors $R_{1}, S_{I_{R_{1}}}=4 \mathrm{kT} / R_{1}$ is their current PSD, and $B W_{n}$ is the noise equivalent bandwidth at the ZCD output.

Regarding the tail of the first stage, a similar reasoning as [112] can be followed, with the main difference due to the peculiar ramp-shape of the ZCD inputs. Before the beginning of the ramps, when the stage is unbalanced, all the tail current as well as its noise (with PSD $S_{\mathrm{I}_{\mathrm{T}_{1}}}$ in Fig. 5.16) is steered entirely to one side, thus only one output of the first stage is affected by noise, with voltage variance $S_{I_{T_{1}}} R_{1}^{2} B W_{n}$. During the input ramps, the tail current is gradually re-distributed to bias both MOSFETs of the differential couple. When the zero-crossing is reached, the tail current is distributed equally between the two transistors of the differential stage. Now the noise contribution at the differential output of the first stage is zero, because the two outputs exhibit the same noise from the tail current source (common mode fully correlated noise). At the same time, during the ramps, the voltage variance $S_{I_{T_{1}}} R_{1}^{2} B W_{n}$ accumulated during the unbalanced state decays exponentially with time constant $R_{1} C_{1} / 2$ [112], where $C_{1}$ is the total output capacitance of the first stage, as shown in Fig. 5.16. However, as the time needed for the output crossing is much higher than the time constant, there is hardly any net contribution left at the crossing time.

Referring to Fig. 5.16, we still have to discuss the noise contribution of the differential pair in the ZCD first stage and the noise generated in the ZCD second stage. As for the differential pair in the ZCD first stage, around the crossing point, both the MOSFETs are on and contribute to the output noise. If $i_{n}$ is the current fluctuation in each MOSFET of the 
Table 5.1: VALUES OF THE VARIABLES SHOWN IN FIG. 5.16 ASSOCIATED WITH THE PROTOTYPE UTDUCK.

\begin{tabular}{|c|c|}
\hline Variable & Value \\
\hline$S_{I_{1}}$ & $60.8 \mathrm{e}-24 \mathrm{~A}^{2} / \mathrm{Hz}$ \\
\hline$S_{I_{2}}$ & $41.5 \mathrm{e}-24 \mathrm{~A}^{2} / \mathrm{Hz}$ \\
\hline$S_{I_{C}}$ & $22.6 \mathrm{e}-23 \mathrm{~A}^{2} / \mathrm{Hz}$ \\
\hline$S_{I_{R}}$ & $30.9 \mathrm{e}-24 \mathrm{~A} 2 \mathrm{~Hz}$ \\
\hline$S_{I_{T_{2}}}$ & $56.1 \mathrm{e}-24 \mathrm{~A}^{2} / \mathrm{Hz}$ \\
\hline$S_{I_{T_{R}}}$ & $62.7 \mathrm{e}-24 \mathrm{~A} 2 / \mathrm{Hz}$ \\
\hline$C_{\mathrm{DAC}}$ & $1.82 \mathrm{pF}$ \\
\hline$C_{L}$ & $1.24 \mathrm{pF}$ \\
\hline
\end{tabular}

\begin{tabular}{|c|c|}
\hline Variable & Value \\
\hline$C_{1}$ & $7 \mathrm{fF}$ \\
\hline$C_{2}$ & $20 \mathrm{fF}$ \\
\hline$R_{1}$ & $960 \Omega$ \\
\hline$R_{2}$ & $2 \mathrm{k} \Omega$ \\
\hline$g_{m_{1}}$ & $7.1 \mathrm{mS}$ \\
\hline$g_{m_{2}}$ & $1.0 \mathrm{mS}$ \\
\hline$g_{m_{C}}$ & $29.1 \mathrm{mS}$ \\
\hline$S L_{\text {out }}$ & $30 \mathrm{GV} / \mathrm{s}$ \\
\hline
\end{tabular}

couple, corresponding to a PSD $\mathrm{S}_{\mathrm{I}_{1}}$ in Fig. 5.16, the portion of the noise current injected into the load $R_{1}$ is $1 / 2$. Therefore, the transfer of the PSD $S_{I_{1}}$ to the load $R_{1}$ is $(1 / 2)^{2}=1 / 4$. This noise current produces across $R_{1}$ a noise voltage variance that increases exponentially, with a time constant $\mathrm{R}_{1} \mathrm{C}_{1} / 2$ [112]. As the ZCD first stage is high bandwidth, the noise across $R_{1}$ at the crossing instant has reached its asymptotic value. Thus, the total voltage variance at the differential output of the second stage due to the MOSFET pair of the first stage is

$$
\sigma_{v_{\mathrm{ZCD}_{1 \mathrm{P}}}}^{2}=2 \mathrm{~S}_{\mathrm{I}_{1}}\left(\frac{1}{2}\right)^{2} \mathrm{R}_{1}^{2} \frac{1}{4 \mathrm{R}_{1} \mathrm{C}_{1}} \mathrm{~g}_{\mathrm{m}_{2}}^{2} \mathrm{R}_{2}^{2}=\mathrm{S}_{\mathrm{I}_{1}} \frac{\mathrm{R}_{1}}{8 \mathrm{C}_{1}} \mathrm{~g}_{\mathrm{m}_{2}}^{2} \mathrm{R}_{2}^{2}
$$

Where the factor 2 on the left hand side of (5.11) is due to the independence of the two noise generators in the differential couple and $S_{I_{1}}=4 \mathrm{kT} \gamma \mathrm{gm}_{\mathrm{m}_{1}}$.

As for the noise generators in the second ZCD stage, the voltage variance at the output due to the load resistors $R_{2}$ is

$$
\sigma_{v_{Z C D_{2 R}}}^{2}=2 S_{I_{R_{2}}} R_{2}^{2} B W_{n}
$$

The tail noise generator in the second stage, with PSD $\mathrm{S}_{\mathrm{I}_{\mathrm{T}_{2}}}$, does contribute to the output noise. In fact, since the inputs of the second stages can be approximated as voltage steps, the tail current can be considered to switch instantaneously from one side of the differential couple to the other one. Therefore, the reasoning made in [112] can be applied, so the total noise variance transferred to the differential output is

$$
\sigma_{v_{\mathrm{ZCD}_{2} \mathrm{~T}}}^{2}=\left(\mathrm{S}_{\mathrm{I}_{\mathrm{T}_{2}}}+\mathrm{S}_{\mathrm{I}_{\mathrm{T}_{\mathrm{R}}}}\right) R_{2}^{2} \mathrm{BW}_{n}
$$

Where the current PSD $S_{\mathrm{I}_{\mathrm{T}}}$ of the reference side in the tail mirror (with ratio $1: 1$, not shown in Fig. 5.16) has been also included. 
Table 5.2: VOLTAGE VARIANCES DUE TO NOISE, CALCULATED FROM (5.7) - (5.14) AND CORRESPONDING JITTER USING (5.6).

\begin{tabular}{|c|c|c|}
\hline $\begin{array}{c}\text { Voltage } \\
\text { noise }\end{array}$ & Value & $\begin{array}{c}\text { Corresponding jitter } \\
\text { from (5.6) }\end{array}$ \\
\hline$\sigma_{v_{I p}}^{2}$ & $(3.2 \mathrm{mV})^{2}$ & $(107 \mathrm{fs})^{2}$ \\
\hline$\sigma_{v_{I n}}^{2}$ & $(1.2 \mathrm{mV})^{2}$ & $(39 \mathrm{fs})^{2}$ \\
\hline$\sigma_{v_{C}}^{2}$ & $(1.1 \mathrm{mV})^{2}$ & $(38 \mathrm{fs})^{2}$ \\
\hline$\sigma_{v_{\mathrm{ZCD}_{1 R}}}^{2}$ & $(0.9 \mathrm{mV})^{2}$ & $(30 \mathrm{fs})^{2}$ \\
\hline$\sigma_{v_{\mathrm{ZCD}_{1 P}}}^{2}$ & $(2.0 \mathrm{mV})^{2}$ & $(68 \mathrm{fs})^{2}$ \\
\hline$\sigma_{v_{\mathrm{ZCD}_{2 R}}}^{2}$ & $(0.6 \mathrm{mV})^{2}$ & $(21 \mathrm{fs})^{2}$ \\
\hline$\sigma_{v_{\mathrm{ZCD}_{2 T}}}^{2}$ & $(1.7 \mathrm{mV})^{2}$ & $(57 \mathrm{fs})^{2}$ \\
\hline$\sigma_{v_{\mathrm{ZCD}_{2 P}}}^{2}$ & $(0.7 \mathrm{mV})^{2}$ & $(24 \mathrm{fs})^{2}$ \\
\hline
\end{tabular}

Finally, the noise of the differential pair in the second ZCD stage produces an exponential voltage noise across the load resistance $R_{2}$, similarly to the ZCD first stage. As the input slopes of the second ZCD stage are, by design, three times the gate delay of the technology, the time interval to reach their crossing is $\tau \simeq 3 \ln (2) R_{2} C_{2}$, where $C_{2}$ is the total output capacitance of the second stage, shown in Fig. 5.16. Thus the voltage variance at the output is

$$
\sigma_{v_{Z C_{2 P}}}^{2}=\mathrm{S}_{\mathrm{I}_{2}} \frac{\mathrm{R}_{2}}{8 \mathrm{C}_{2}}\left(1-e^{-\frac{3 \ln (2) \mathrm{R}_{2} \mathrm{C}_{2}}{\frac{\mathrm{R}_{2} \mathrm{C}_{2}}{2}}}\right) \simeq 0.98 \mathrm{~S}_{\mathrm{I}_{2}} \frac{\mathrm{R}_{2}}{8 \mathrm{C}_{2}}
$$

The total voltage variance at the differential output of the second ZCD stage can be obtained by summing all the term from (5.7) to (5.14). The noise equivalent bandwidth $B W_{n}$ to be considered in (5.10), (5.12) and (5.13) is determined by the pole of the second ZCD stage:

$$
B W_{n}=\frac{1}{4 R_{2} C_{2}}
$$

By summing the expressions of the voltage variances (5.7) - (5.14), using the values listed in Table 5.1, the total voltage variance at the ZCD output is $\sigma_{v_{\text {out }}}^{2}=(4.7 \mathrm{mV})^{2}$, corresponding to a jitter of $(160 \mathrm{fs})^{2}$, according to (5.6). The single contributions are listed in Table 5.2. Since the variance has been determined during a charge state $(C R)$, leading to the generation of an output edge, the time jitter resulting from (5.6) is an absolute jitter. The period jitter is twice the absolute jitter, as the period is given by the difference between two edges. The expected jitter we have calculated here will be compared to simulations in section 5.6 and with measurements in section 5.7.6. 


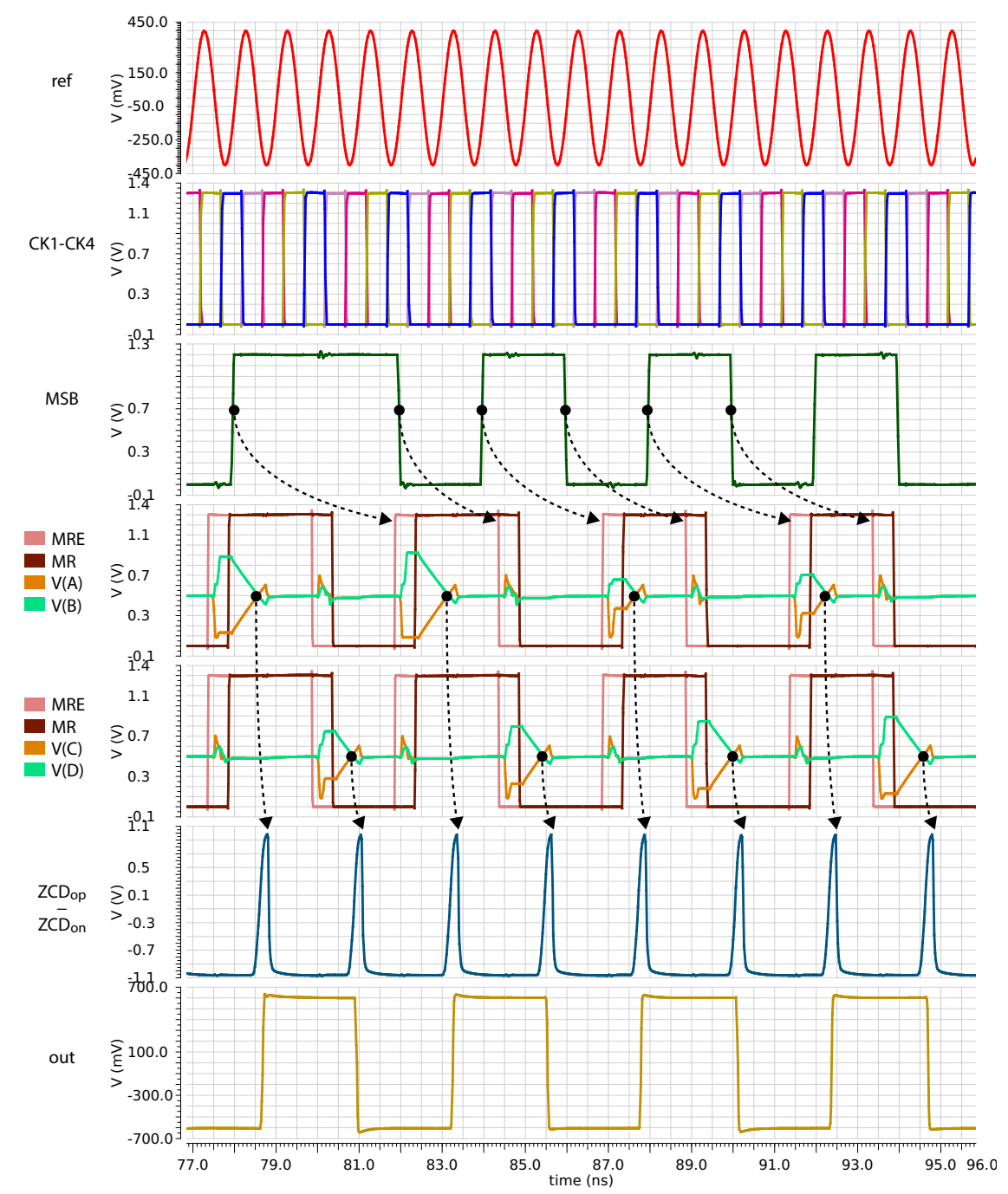

Figure 5.18: Simulation of the DFC prototype, with $f_{\text {ref }}=1 \mathrm{GHz}$ and $\mathrm{FCW}=1792$. Bondwires modeled with inductors of $\mathrm{L}=2 \mathrm{nH}$. Signal names according to Fig. 5.1, Fig. 5.4, Fig. 5.5, Fig. 5.12, Fig. 5.13. The dashed arrows show the cause and effect of signals.

\subsection{Simulations}

We collected in this section most of the simulations carried out during the design process of the prototype to support the design choices discussed in the previous sections. For every simulation we will refer back to the related design section.

Fig. 5.18 shows the basic operation of the prototype UTDUCK as DFC, by plotting the input and the output waveforms, as well as the main internal signals of the prototype. The input reference frequency and the frequency control word chosen as an example for the simulation are $f_{\text {ref }}=$ 


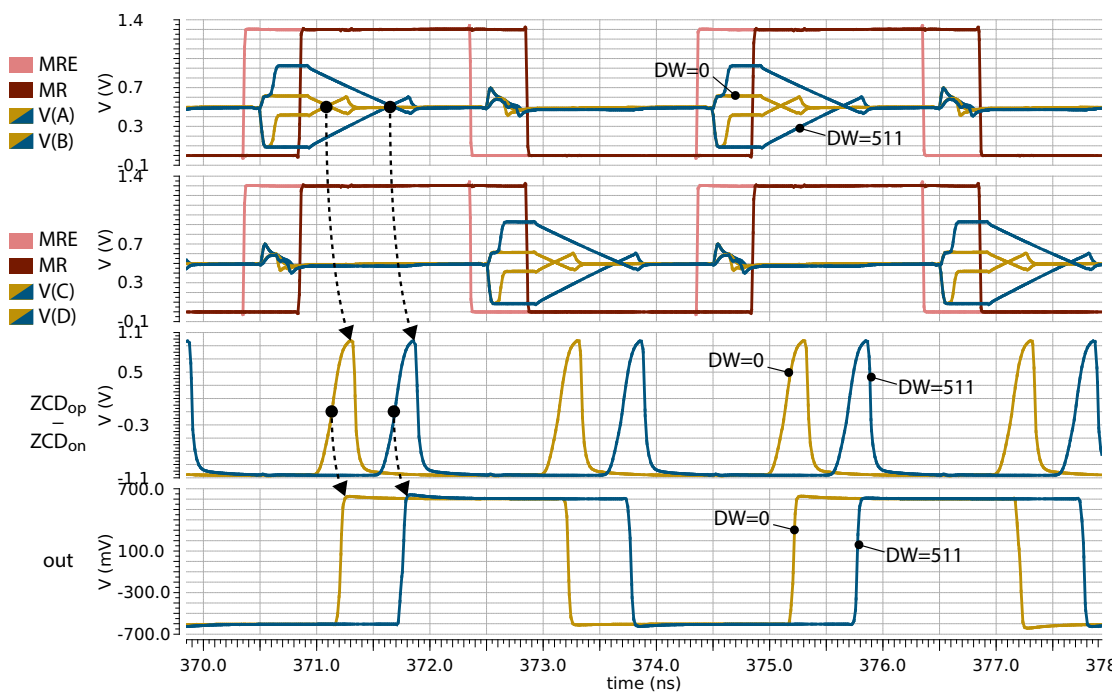

Figure 5.19: Fine-delay added by the DTC, for the two extreme values of DW $\left(0\right.$ and 511) and $f_{\text {ref }}=1 \mathrm{GHz}$. Simulations with FCW $=2048$. The dashed arrows show the cause and effect of signals.

$1 \mathrm{GHz}$ and FCW $=1792$, respectively. These values allow to show the operation of the DFC prototype when FCW is not a power of two, so the effect of the DTC is easily visible. Moreover, with that choice of FCW, the DTC operates twice at every clock edge, so it is an example at high DTC speed. The simulation results reflect the expected behaviour of the DFC, previously described in chapter 4 . Starting from the input reference clock (first row in Fig. 5.18), the four clock phases are derived (second row). The resulting MSB of the counter (third row) has a wider pulse next to other narrow pulses, indicating that its instantaneous frequency is not constant. The MSB of the counter is then resampled with one of the clock phases according to the coarse DW, thus producing the resampled signals MRE and MR (fourth row in Fig. 5.18), which include the DTC coarse delay only. MRE starts the DAC conversion, which set the starting levels of the complementary ramps (different levels at each edge of MRE), while MR determines the ramps' start. Note that for the case FCW $=1792$ the DTC code is different at every edge of MR. The ramps' crossing is then detected by the ZCD, whose differential output is shown in the sixth row of Fig. 5.18. The resulting DFC output is obtained by dividing by 2 the frequency of the ZCD output and by buffering it to the $50 \Omega$ load. The DAC conversion is allowed to go on during the ramps, as it is superimposed to the ramp generation. However, the DAC conversion should be ended before the crossing instant to achieve a linear control of the crossing.

The simulation in Fig. 5.19 aims to show the effect of the DTC fine-delay, discussed in section 5.1 and section 5.3. It has been done with FCW $=2048$ (a power of 2), so that the instantaneous frequency of MRE and MR is 


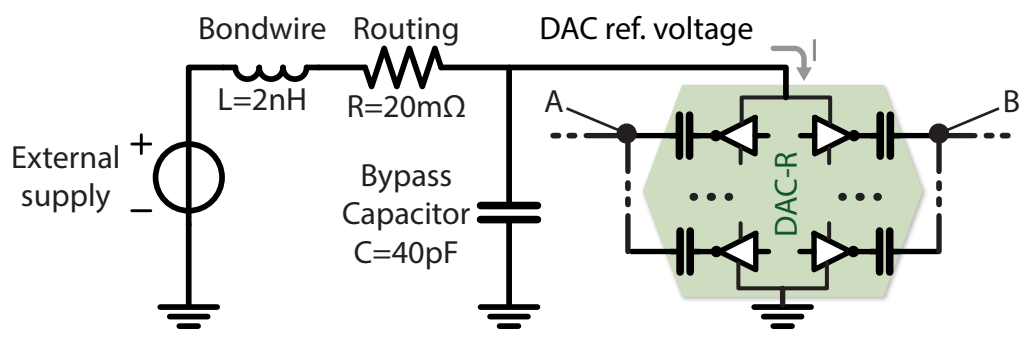

(a) Circuit model.

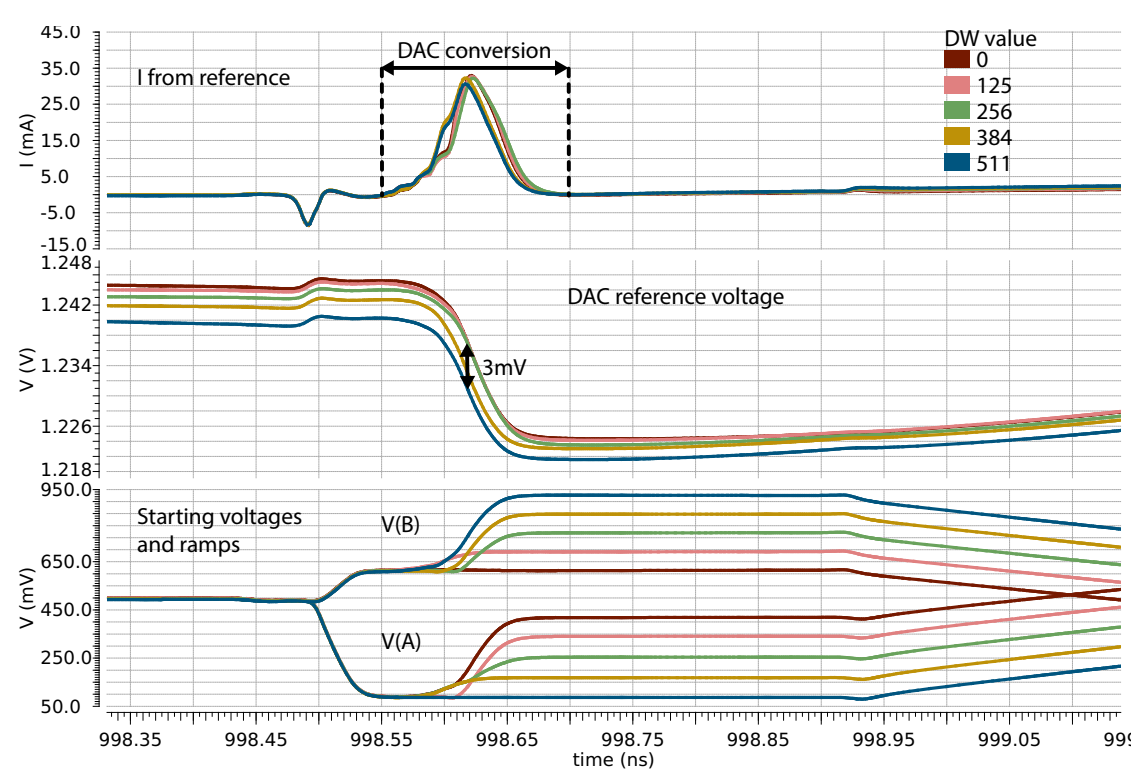

(b) Waveforms.

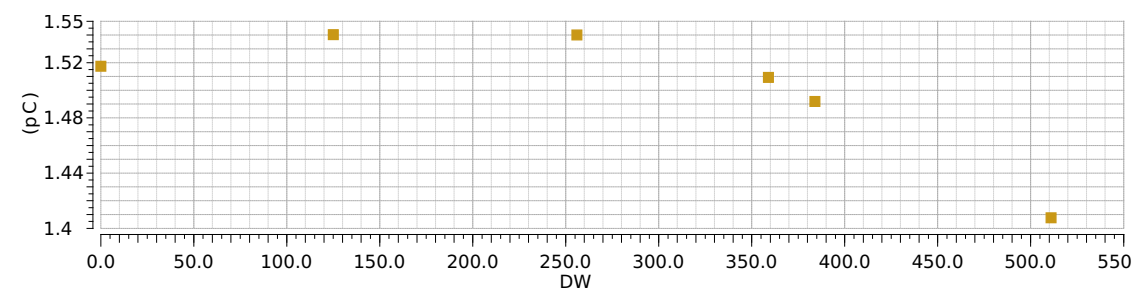

(c) Integrated peak of current as function of DW.

Figure 5.20: Simulated behavior of the DAC reference supply for different values of DW. Simulation made with $f_{\text {ref }}=1 \mathrm{GHz}$ and FCW $=2048$. $\mathrm{DW}=0,125,256,384,511$. (a) Circuit model. (b) Current drawn from the reference supply during the DAC conversion and corresponding voltage waveform. The voltage ramps are also shown for reference. (c) Integrated peak of current for the same values of DW considered in (b). 


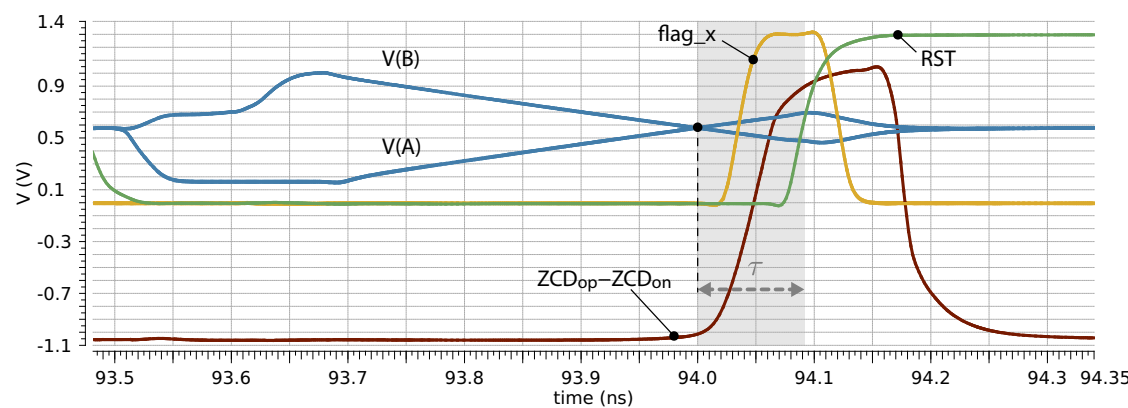

Figure 5.21: Start of the reset state RST after the crossing. RST signal triggered by flag_x. Simulation with $f_{\text {ref }}=2 \mathrm{GHz}, \mathrm{FCW}=2048$ and $\mathrm{DW}=511$.

already the target one, while the fine-DW of the DTC has been forced to its minimum and maximum values, 0 and 511 respectively, to show the effect on the ramps and on the output. Notice that the system in Fig. 5.19 is not working as DFC, thus the values of the fine-DW are not related to FCW. The purpose here is to force two fixed values of DW in two distinct simulation runs, in order to isolate the effect of the fine DTC. Each value of DW (0 or 511) determines the starting voltages of the complementary ramps (first and second rows in Fig. 5.19), thus controlling the crossing instant and, ultimately, the fine-delay of the output (last row in Fig. 5.19).

In conclusion, the nine least significant bits of DW make a fine control of the edges at the DTC output. The simulations have shown the total shift in the output edges due to the two extreme DW values with nine bits (0 and 511). In the normal system operation as a DFC, the DW can vary at every clock period at most. A calibration procedure will ensure that the fine full-scale delay, i.e. the shift in the output edges in Fig. 5.19, will match the coarse delay-step, that is the delay between two adjacent clock phases. This can be done by controlling the ramp's slope with a tunable current ( $\mathrm{I}_{\text {ref core }}$ shown in Fig. 5.8). Calibration will be discussed in section 5.7.2 and with more details in appendix A.3.

Fig. 5.20 shows the DAC reference voltage and current during the conversion for different codes: $\mathrm{DW}=0,125,256,384,511$. The goal is to show their code-independency during the DAC conversion with the custom driving scheme discussed in section 5.3.6. The current drawn from the DAC reference voltage is shown in first row of Fig. 5.20b. The slight shape variation with different codes DW is due to a skew among different bit lines of DW. The current peak, integrated from $998.55 \mathrm{~ns}$ to $998.70 \mathrm{~ns}$, varies by $10 \%$, from $1.41 \mathrm{pC}$ to $1.54 \mathrm{pC}$, in the range of $\mathrm{DW}$ considered. This causes a variation of the DAC reference voltage of $3 \mathrm{mV}$ with code during the DAC conversion, as shown in the second row in Fig. 5.20b. This simulated code-dependency of the reference voltage causes an expected error of max 2 LSB in the produced voltage levels, when considering the 


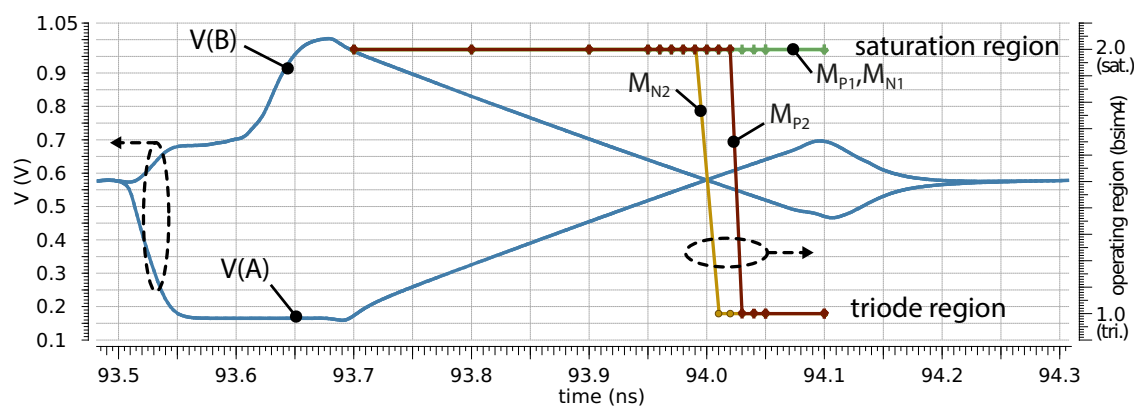

Figure 5.22: Operating region of the current source transistors during the ramps, showing the saturation region of the current sources until the ramps' crossing. Simulation with $f_{\text {ref }}=2 \mathrm{GHz}, \mathrm{FCW}=2048$ and DW $=511$.

values of capacitances in table Table 5.1.

Fig. 5.21 is related to the state diagram of the DTC core, discussed in section 5.3.4. The goal of this simulation is to support the design choice of triggering the signal RST with flag_ $x$, rather than the ZCD output. This choice, due to speed considerations, has been motivated in section 5.3.4 and can be validated with Fig. 5.21, which shows the rising of RST. The time delay between the ramps' crossing and RST can be named $\tau$, highlighted in gray in Fig. 5.21. ${ }^{\dagger}$ It is one of the bottlenecks of the system speed: it should be kept short to speed up the circuit (see section 5.5.1), but long enough to guarantee a full transition low-high of the ZCD output. The signal flag_x switches 20 ps before the ZCD output, as can be seen in Fig. 5.21. However flag_x is actually dependent on the rising ramp only, rather than on the crossing point of the two ramps, since flag_x is obtained from inverters placed at the ZCD inputs (see Fig. 5.5). Nonetheless, since $\tau$ is long enough to allow a full switch of the ZCD output (its rising edge is fully contained inside the interval $\tau$ in Fig. 5.21), the choice of flag_ $x$ to trigger the signal RST allows to reduce the delay $\tau$ by a few tens of ps without affecting the path through the ZCD. In conclusion, the signal flag_ $x$ can quickly trigger the reset state, while the highly linear detection of the ramps' crossing is provided by the path through the ZCD.

Fig. 5.22 shows the operating region of the transistors of the complementary current sources during the ramps. The goal is to show that the current sources operate in saturation region for the entire duration of the ramps, until the crossing time, even in the worst case of DW $=511$, where the voltage excursion during the ramps is maximum. The names used in Fig. 5.22 refer to Fig. 5.8. The simulation refers to the DTC fine R, but the same results hold for the interleaved one. As can be seen in Fig. 5.22, transistors $M_{P 1}$ and $M_{N 1}$ stay in saturation for the entire duration of the ramps, while the cascodes $M_{\mathrm{P} 2}$ and $M_{\mathrm{N} 2}$ go to the triode region just after

\footnotetext{
$\dagger$ Notice that $\tau$ has been expressed as a sum of gate delays in section 5.5.1. The correspondance is $\tau=2 \tau_{\text {gate }}+\tau_{\mathrm{FF}}$ and this sum appears in the speed condition (5.3).
} 


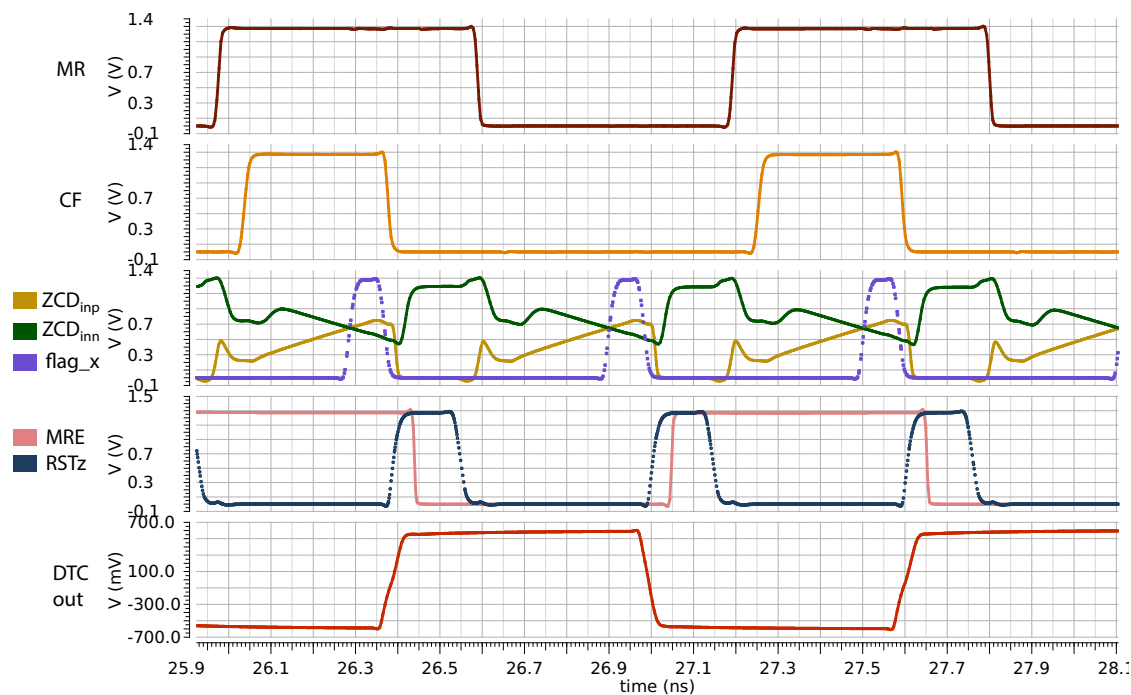

(a) $f_{r e f}=3.3 \mathrm{GHz}$.

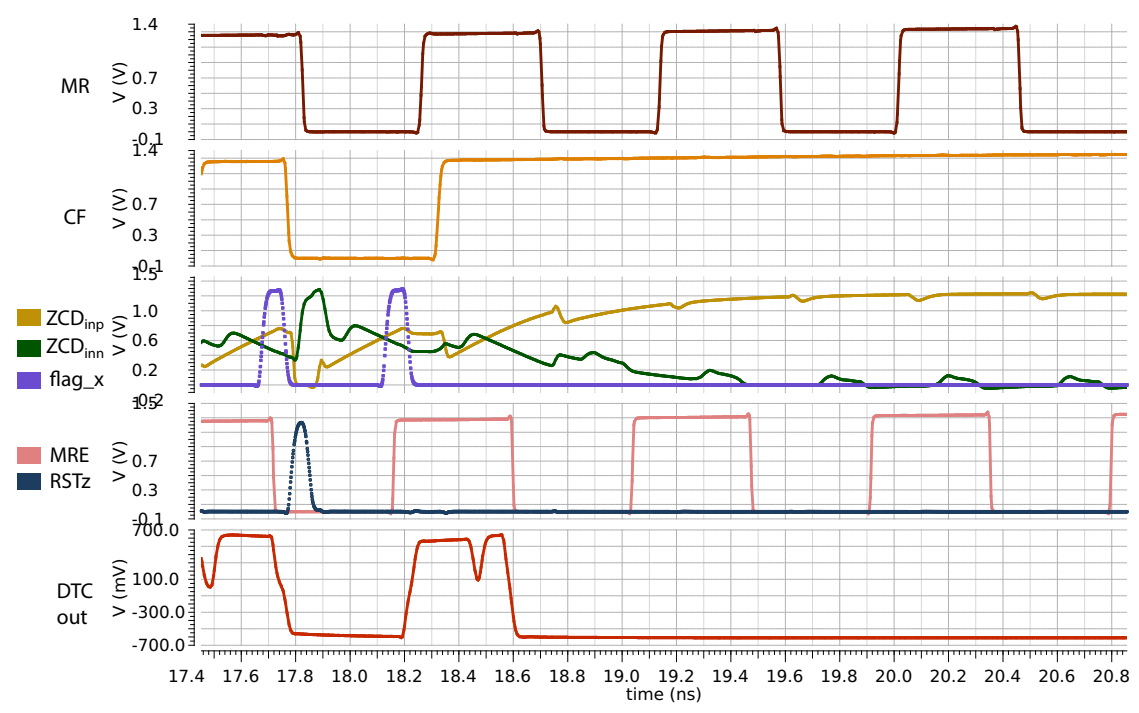

(b) $\mathrm{f}_{\text {ref }}=4.5 \mathrm{GHz}$ (time violation).

Figure 5.23: Simulations demonstrating the critical condition for maximum speed in the DFC. In (a) the DFC operation is persistent. In (b) condition (5.2) is not satisfied and the DFC stops working. Both simulations with $\mathrm{FCW}=2048$ and $\mathrm{DW}=511$.

the crossing instant, as intended.

Fig. 5.23 shows the validity of the critical condition (5.2) which sets the maximum speed of the DFC, as discussed in section 5.5.1. In that section, the upper limit $3.57 \mathrm{GHz}$ for the reference frequency has been calculated (5.4), thus the simulations in Fig. 5.23 show two cases below and beyond that limit. Both simulations refer to the worst case of fastest programmed 
output $(F C W=2048)$ and maximum fine-delay $(D W=511)$. Fig. 5.23a shows the case with $f_{\text {ref }}=3.3 \mathrm{GHz}$. After the ramps' crossing during the charging state $C R$, the reset signal $R S T_{z}$ rises up before the edge of MRE (fourth row in Fig. 5.23a). In this way, the ZCD can be correctly unbalanced before the action of the DAC (see the ZCD inputs in the third row in Fig. 5.23a). The output produces stable DFC operation at the target frequency $\left(f_{\text {ref }} / 4=825 \mathrm{MHz}\right)$. Fig. $5.23 \mathrm{~b}$ shows the case with $\mathrm{f}_{\text {ref }}=4.5 \mathrm{GHz}$. At the beginning of the transient simulation the reset signal $\mathrm{RST}_{z}$ can still go high but its rising edge occurs after the falling edge of $M R E$, thus violating (5.2). The next ramp can still occur but then the reset signal does not switch anymore, thus causing the two inputs of the ZCD to gradually diverge to the rail levels. Therefore, no correct output is produced. The highest input frequency producing a stable oscillation in simulations is $f_{\text {ref }}=4 \mathrm{GHz}$.

Fig. 5.24 shows the Integral Non-Linearity (INL) of one fine-DTC. Both the ramps' crossing points (Fig. 5.24b) and the zero crossings of the final output (Fig. 5.24c) show a peak INL below 2 LSB. This non linearity is likely due to the DAC error in the starting levels of the ramps, discussed when referring to Fig. 5.20.

Fig. 5.25 shows the results of a time domain noise simulation of the DFC. The goal is to verify the total voltage variance at the ZCD output calculated in section 5.5.2. The simulation has been executed using a Verilog-A model of the digital circuit, since the original Verilog-AMS model used in the actual design is not compatible with a periodic-steady-state analysis using Spectre RF. The values used in the simulations are $f_{\text {ref }}=2 \mathrm{GHz}$ and FCW $=$ 2048 , thus the output period is 2 ns. Besides the voltage waveforms in one output period, Fig. 5.25 shows the voltage uncertainty at selected nodes during the same time. Fig. 5.25a considers the noise between nodes $A$ and $B$, while Fig. 5.25 b shows the voltage noise at the ZCD output. In Fig. 5.25a the voltage noise at nodes $A$ and $B$ slightly increases with a constant slope during the ramps, due to the integration of the noise current coming from the current sources. The voltage noise value between nodes $A$ and $B$ at the crossing time is $\sigma_{v_{A-B}} \simeq 262 \mathrm{uV}$. This is consistent with calculations, since the square root of the sum of (5.7), (5.8) and (5.9) (first three rows of Table 5.2), divided by the ZCD gain $R_{1} g_{m_{1}} R_{2} g_{m_{2}}$, leads to $\sigma_{v_{A-B}} \simeq 263 \mu \mathrm{V}$, with the values in Table 5.1. In Fig. 5.25b the noise at the ZCD differential output is maximum during the transition low to high of the output waveform. The simulated maximum value $\sigma_{v_{\text {out }}} \simeq 4.8 \mathrm{mV}$ is consistent with the calculated value $\sigma_{v_{\text {out }}} \simeq 4.7 \mathrm{mV}$ in section 5.5.2, obtained from the square root of the sum of (5.7)-(5.14).

\subsection{Measurements}

The chip is fabricated in $65 \mathrm{~nm}$ low-power bulk CMOS technology by TSMC, operating at $1.2 \mathrm{~V}$. The total area (including bondpads) is $875 \mathrm{um} \times$ $860 \mathrm{um}$. The chip micrograph is shown in Fig. 5.26. The active area ex- 


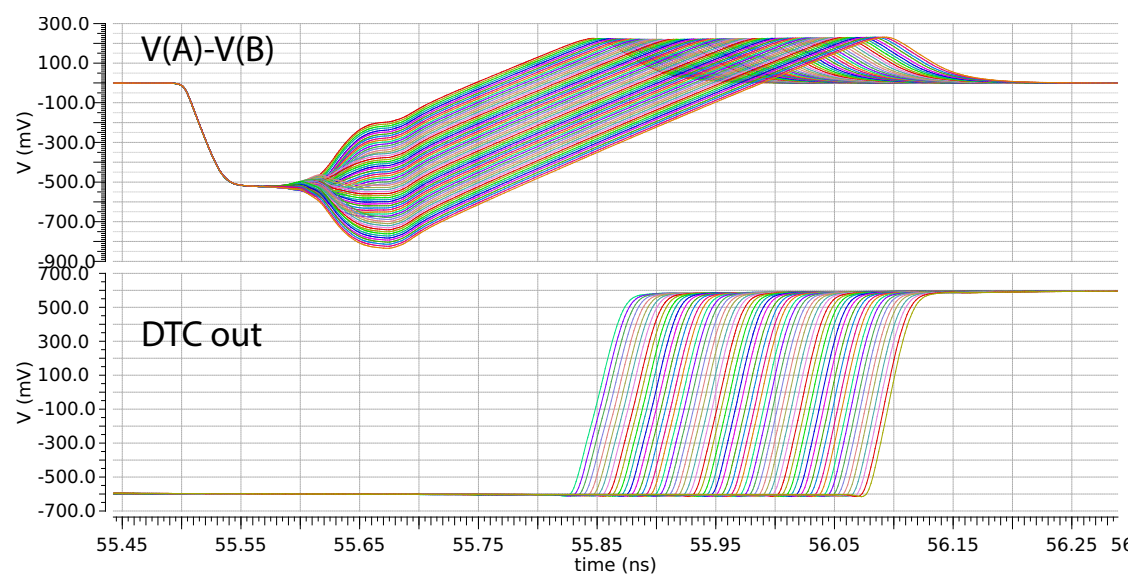

(a) Simulated waveforms: differential ramps and output.

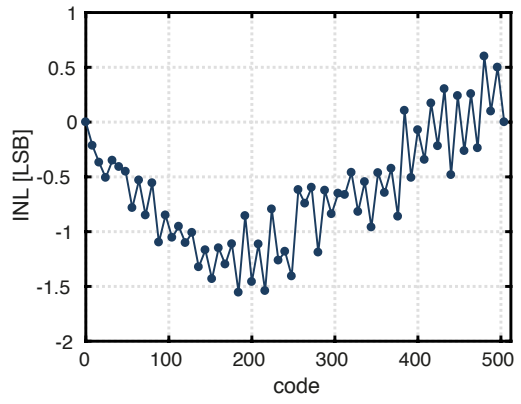

(b) INL of the differential ramps (at $0 \mathrm{~V}$ threshold).

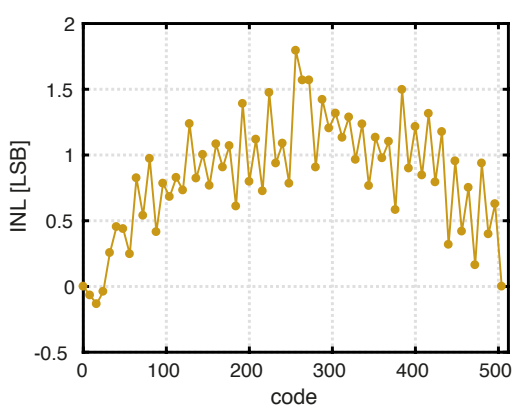

(c) Output INL.

Figure 5.24: Linearity of one fine-DTC. Codes ranging from DW $=0$ to DW $=511$ with step-size 8 for readability. Transient simulations with $f_{\text {ref }}=2 \mathrm{GHz}$ and $\mathrm{FCW}=2048$.

cluding decap is $0.215 \mathrm{~mm}^{2}$. The chip is packaged in a QFN40-package $5 \mathrm{~mm} \times 4 \mathrm{~mm}$.

\subsubsection{Additional circuit for measurement}

Some additional logic in the digital front-end of UTDUCK allows the circuit operation to switch from the DFC behaviour discussed above, which we will name DFC-mode, to another mode of operation useful for measurements, named measurement-mode. The difference is shown in Fig. 5.27. In DFC-mode, the system operates as described in chapter 4, where the current value of DW to be sent to the DTC is calculated inside the chip, starting from the counter output, according to (4.7), and it is updated automatically at every edge of signal MSB. Instead, in measurement-mode, the delay word DW is forced to be equal to an external input code, or it can be enabled to switch between two input codes $D W_{L}$ and $D W_{H}$, digitally controllable externally. The current value of DW depends on the logic level 

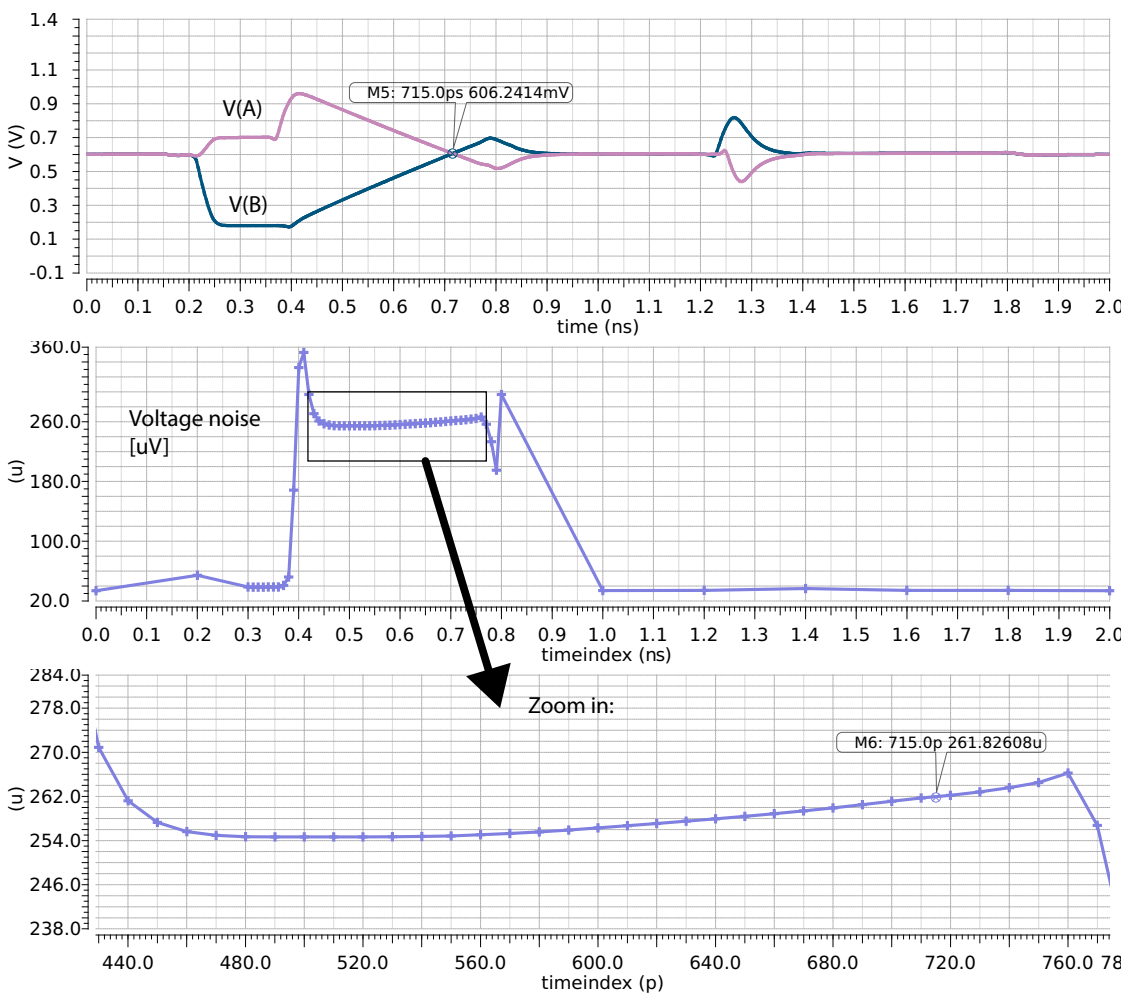

(a) Noise probed at the nodes developing the ramps (nodes A and B).
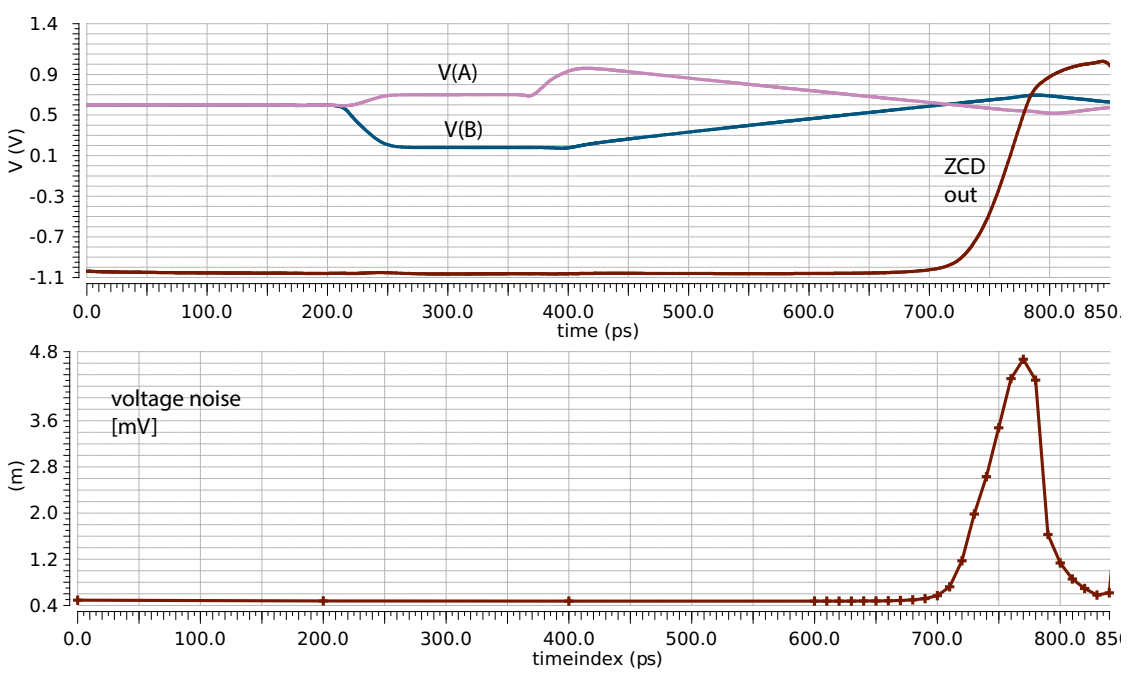

(b) Noise probed at the ZCD output.

Figure 5.25: Simulation results of a time domain noise simulation of the DFC. Integrated noise over time and main waveforms. 


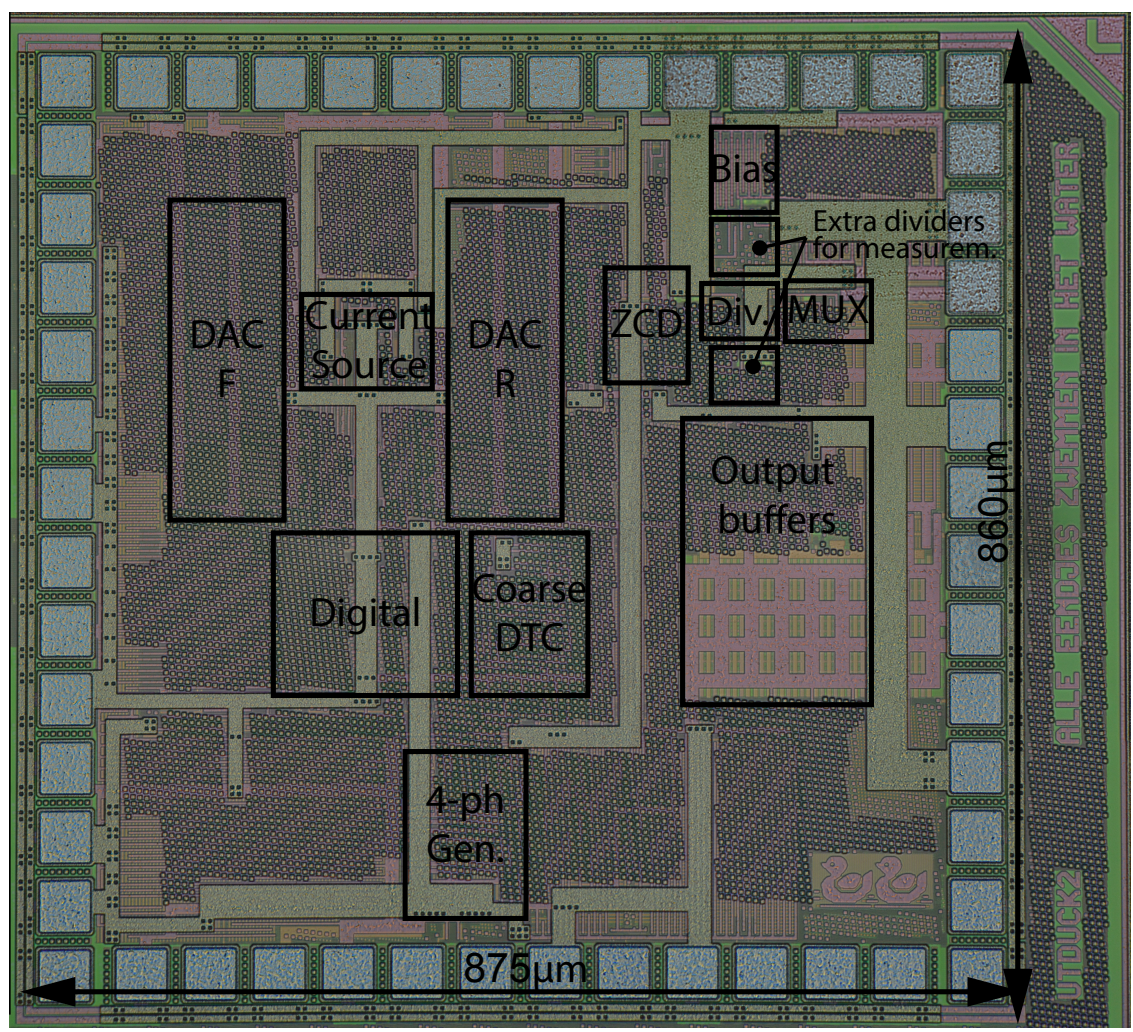

Figure 5.26: Chip micrograph of UTDUCK, implemented in bulk TSMC $65 \mathrm{~nm}$ technology.

of an external modulating clock $\mathrm{CK}_{\text {ext }}$ (see Fig. 5.27). This behavior allows to make INL and time measurements, discussed in section 5.7.3.

Extra dividers-by- 2 are added in series between the ZCD and the output buffers, as shown in Fig. 5.28, to be able to discard the rising or the falling edges of the output. A multiplexer selects the set of edges that should be brought to the output, depending on 2 bits, digitally controllable.

\subsubsection{Calibration}

A calibration is necessary before the measurements to achieve the following goals:

- align the four clock phases relatively to each other, to make their time difference exactly $\mathrm{T}_{\text {ref }} / 2$, as shown in Fig. 5.2;

- equalize the coarse delay added to the rising edges of the waveforms MR and MRN, to achieve a 50\% duty-cycled square-wave at the output;

- match the full-scale delay of the fine DTC to the delay step of the coarse DTC, in order to avoid timing-gaps; 

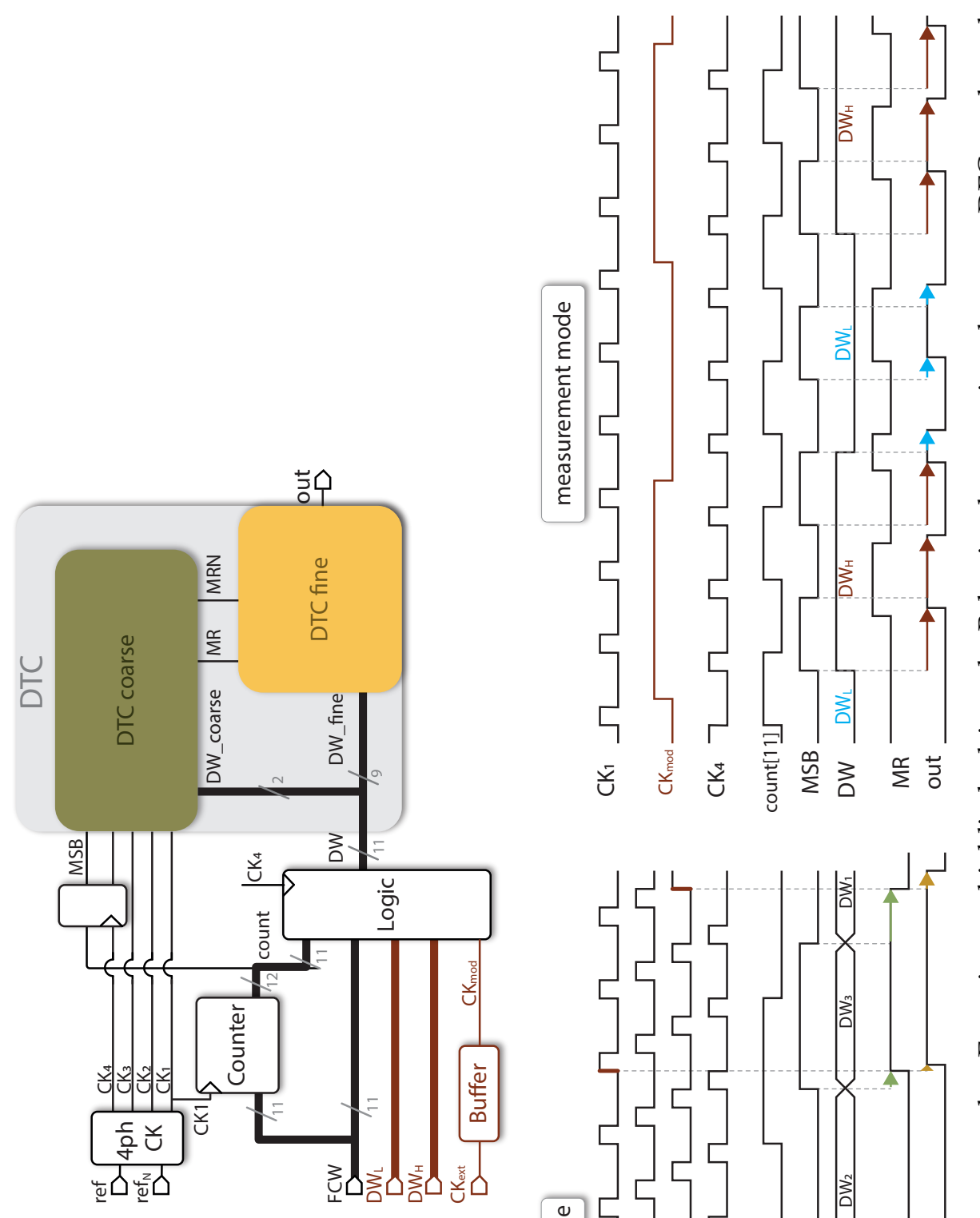

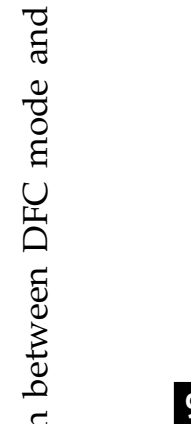

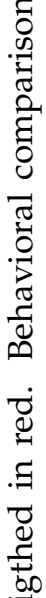

99

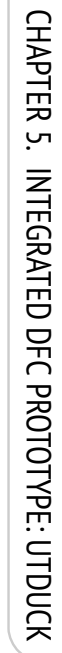

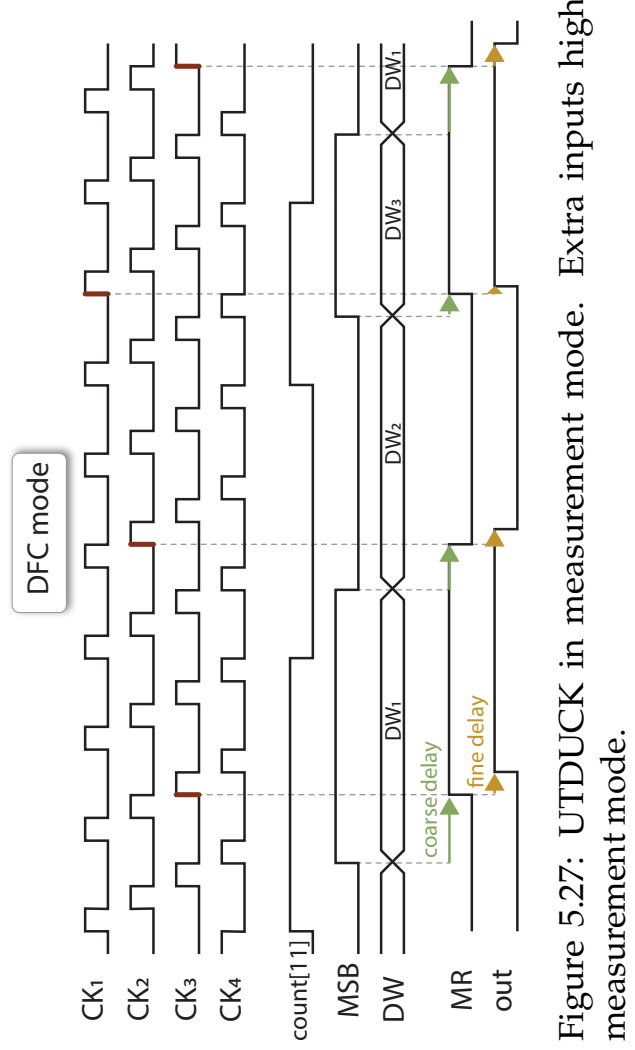




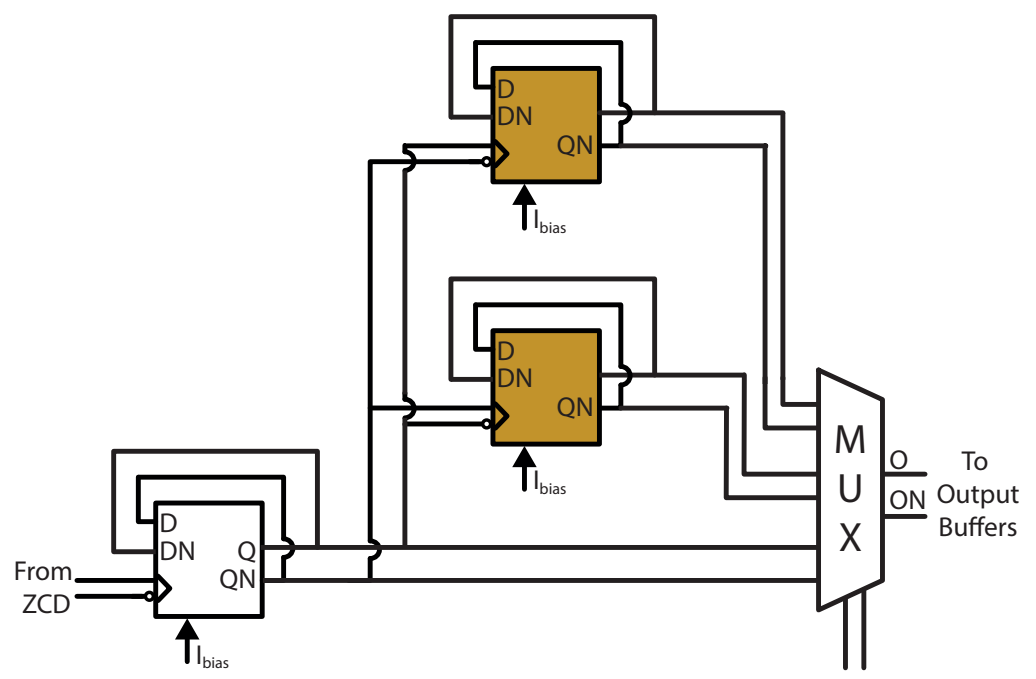

Figure 5.28: Extra dividers (shown in color) added for measurement purposes, implemented with CML flip-flops in closed loop. A multiplexer selects the edges to be carried to the output.

Most of the calibration procedure can be done digitally, acting on the bytes placed for this purpose at different points in the digital part of the prototype. The calibration algorithm is detailed further in appendix A.3.

\subsubsection{Measurements on the DTC}

The goal of the measurements on the DTC is to verify its linearity, by measuring the delays corresponding to each control code. The delay measurements have been done using the method described in chapter 3 [CP:1], which detects phase modulation, producing sideband tones whose strength is related to delay variations. The spectrum analyzer that has been employed to measure the modulation sidebands is Keysight PXA N9030A. The circuit is set in measurement-mode and the input word FCW is set to a power of 2, so that the MSB of the counter is a pure square that can be used as a carrier, while the DTC generates the phase modulation. One path with extra divider (Fig. 5.28) is selected, to discard the rising (or the falling) edges of the output. In this way it is possible to isolate the edges coming from a single fine-DTC, $\mathrm{R}$ or $\mathrm{F}$, for characterization.

If $\mathrm{T}_{\text {carrier }}$ is the period of the carrier subject to phase modulation, a more general version of (3.2) is

$$
\mathrm{P}_{\text {spur }}=20 \log _{10}\left(2 \frac{\tau}{\mathrm{T}_{\text {carrier }}}\right) \quad[\mathrm{dBc}]
$$

Where $\tau$ is the time delay associated with the modulation spur. Equation (5.16) becomes (3.2) for $\mathrm{T}_{\text {carrier }}=2 \mathrm{~T}_{\mathrm{CK}}$, however (5.16) is preferable for the prototype UTDUCK since the ratio $\mathrm{T}_{\text {carrier }} / \mathrm{T}_{\mathrm{CK}}$ can be programmed 


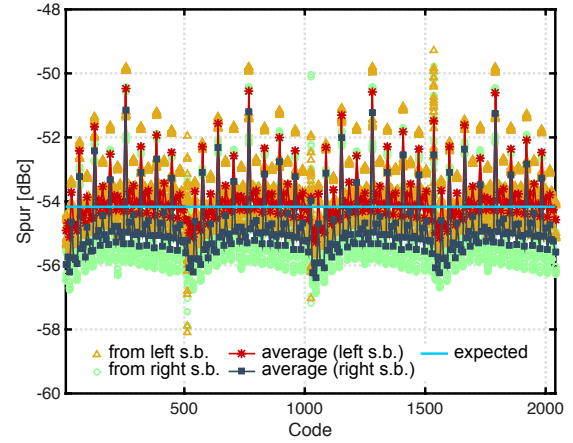

(a) Sidebands, side $R$

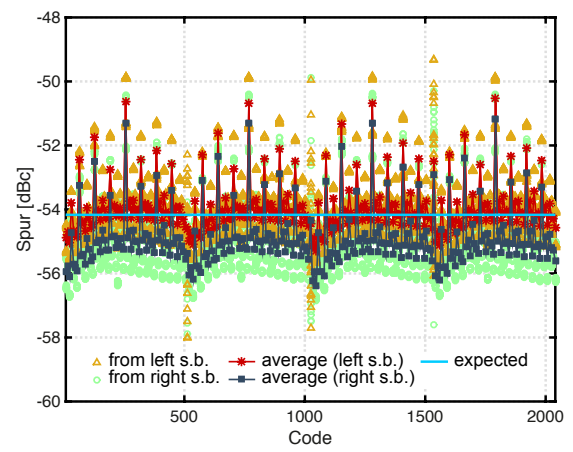

(c) Sidebands, side F

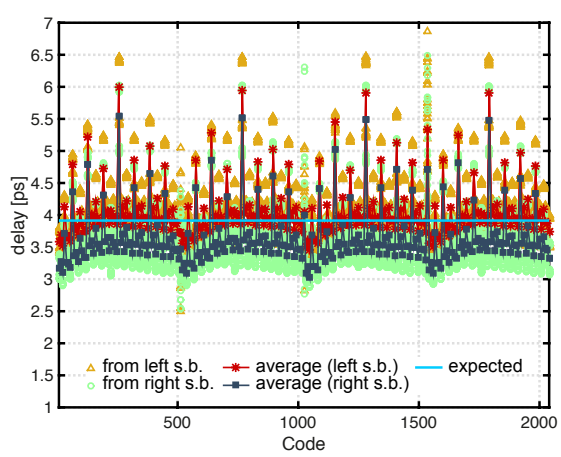

(b) Delays, side $R$

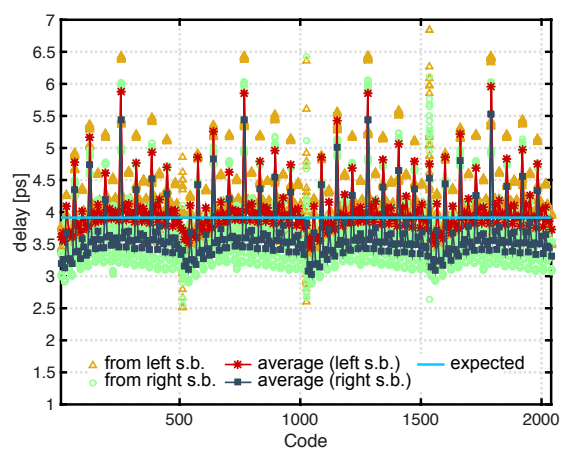

(d) Delays, side F

Figure 5.29: Measured modulation sidebands and corresponding delays as function of the code in UTDUCK. Measurements with $f_{\text {ref }}=2 \mathrm{GHz}$,

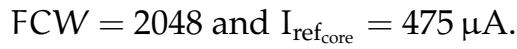

with the input word FCW, being, from (4.1):

$$
\mathrm{T}_{\text {carrier }}=\frac{2^{\mathrm{N}}}{\mathrm{FCW}} \mathrm{T}_{\mathrm{CK}}
$$

The minimum delay that is visible with the measurement method corresponds to a modulation spur equal to the noise floor (which is a function of the chosen resolution bandwith of the spectrum analyzer). Therefore, the measurement time-resolution $\tau_{\text {res }}$ can be obtained from (5.16) by equaling the noise floor of the spectrum analyzer (in $\mathrm{dBc}$ ) to the modulation spur. Thus, it is

$$
\tau_{\text {res }}=\frac{T_{\text {carrier }}}{2} 10^{\text {noise floor }[\mathrm{dBc}] / 20}
$$

The measurements are done with code-steps of 8 to reduce the measurement time and keep the sensitivity high: from (3.7), with a code-step c_step $=8$, a delay uncertainty of 1 LSB would correspond to a sideband variation of $1 \mathrm{~dB}$, so it would be clearly visible on the spectrum analyzer. The resolution bandwidth of the spectrum analyzer is set to $1 \mathrm{kHz}$, corresponding to a noise floor of $-125 \mathrm{dBm}$. The carrier has a power of $-2 \mathrm{dBm}$ 


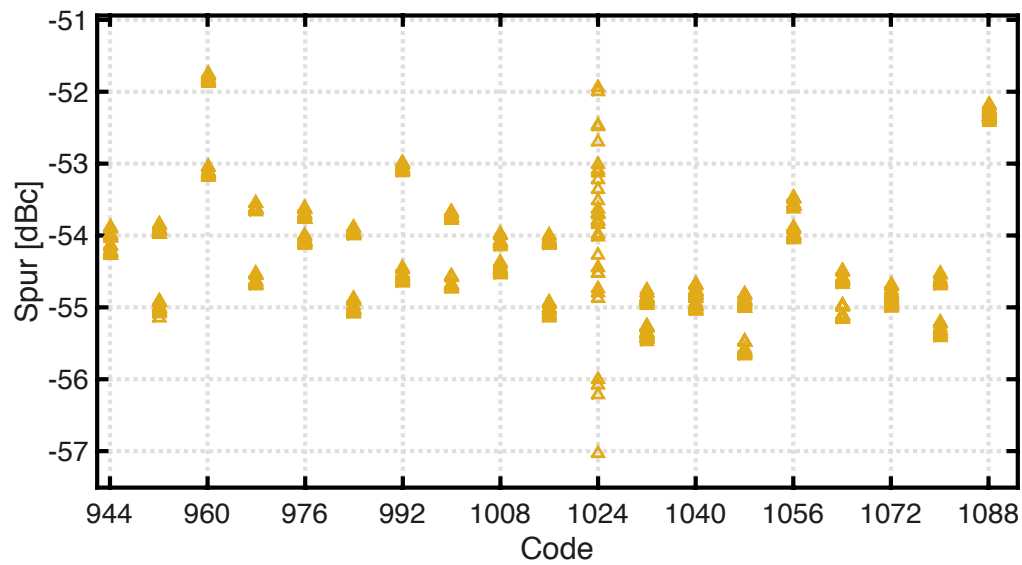

(a) Zoom of the measured left sideband from Fig. 5.29a.

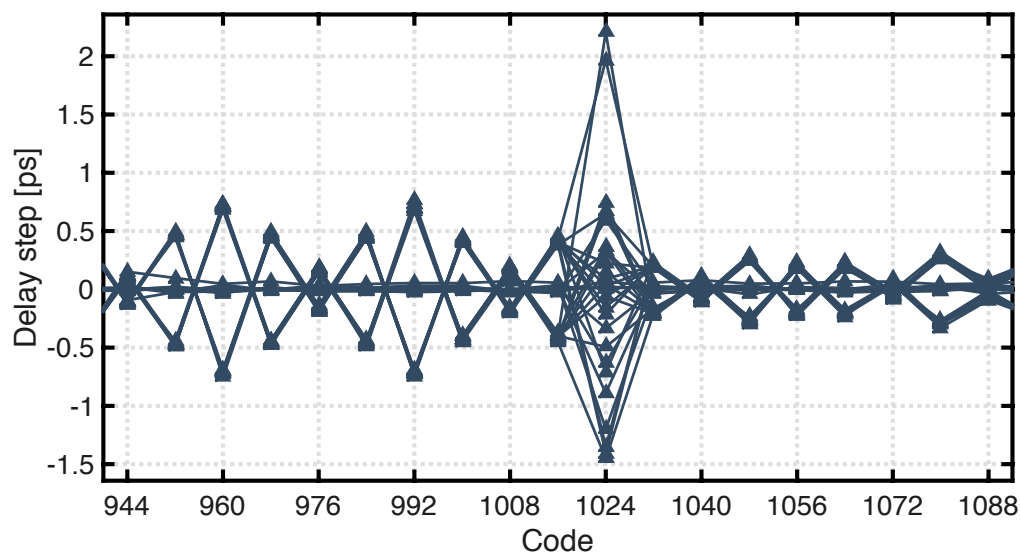

(b) Difference in the produced delay between consecutive sweeps.

Figure 5.30: Jumps in the measured spurs and corresponding jumps in the produced delay.

and frequency of $250 \mathrm{MHz}$. The chosen modulation frequency is $2.5 \mathrm{MHz}$. With these values, the measurement time resolution from (5.18) is only $1.42 \mathrm{fs}$, due to the narrow resolution bandwidth chosen in the spectrum analyzer (and consequently a low noise floor).

Fig. 5.29 shows the measured modulation sidebands and the corresponding delay-steps from (5.16), for both the fine-DTCs R and F. The average value (calculated in linear scale) for each code is plotted as well, to show the trend. Each point has been obtained by switching DW between a couple of values, differing by c_step $=8$, that would result in expected constant sidebands of $-54.2 \mathrm{dBc}$, and therefore $3.9 \mathrm{ps}$, according to (3.2), (3.6), and (5.16). Deviations from this expected constant value are due to the DTC non-linearity. The sweep throughout the entire range of codes has been repeated 30 times. During these iterations, jumps in the measured 


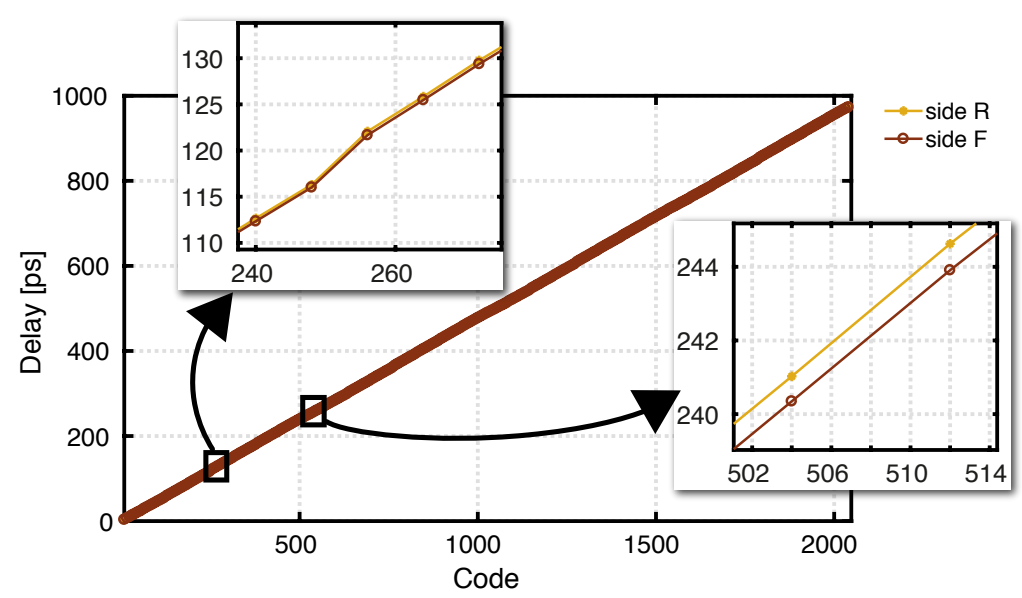

103

Figure 5.31: Measured DTC delay as a function of code. DTC coarse+fine. Measurements with $\mathrm{f}_{\text {ref }}=2 \mathrm{GHz}, \mathrm{FCW}=2048$ and $\mathrm{I}_{\text {ref }_{\text {core }}}=475 \mu \mathrm{A}$.

spurs have been observed. This is better shown in Fig. 5.30a, where measured left sideband from Fig. 5.29a are zoomed in. It can be observed that for codes 512, 1024 and 1536 the measured spurs spread over a $5 \mathrm{~dB}$ interval (corresponding to around 2 ps and 4LSB), while for the other codes the jump is between two distinct values, corresponding to jumps of maximum $0.7 \mathrm{ps}$. The 4 LSB delay variation for codes 512,1024 and 1536 can be due to a metastability condition in the sampling of the fine-DW with MRE and MREN. Instead the jump between always the same two values, that has been observed for the other codes, can be due to glitches in the fine-DW at the time of the sampling edges of MRE and MREN. These jumps were not expected and can be the effect of timing violations between the DTC coarse delay block (producing MRE) and DFC digital core (producing the current DW).

By integrating the delay-steps in Fig. 5.29, we can plot the DTC delay as a function of the code DW, as shown in Fig. 5.31. For each fine-DTC (side $\mathrm{R}$ and side $\mathrm{F}$ ), the delay-steps measured from the left and right sidebands has been averaged. The measurements have been done with $f_{\text {ref }}=2 \mathrm{GHz}$, so the required DTC full-scale (coarse+fine) is $1 \mathrm{~ns}$. The measured full-scale is $20 \mathrm{ps}$ less than the expected one, corresponding to an error in the fine full-scale of around 10 LSB compared to the expected theoretical value $\mathrm{T}_{\text {ref }} / 2=500 \mathrm{ps}$. This is confirmed by measuring the delay at $\mathrm{DW}=504$ (the highest code available for the fine-delay in the set of measurements): it is $240.3 \mathrm{ps}$, that is around $6 \mathrm{LSB}$ smaller than the expected value $245.9 \mathrm{ps}$. This error can be reduced by iterating the calibration steps, however the aforementioned jumps in the measured spurs make any calibration refining quite challenging without clear improvement in the results. Thus we stop at one calibration run and accept the calibration results. The measured mismatch of the fine full-scale between side $R$ and $F$ is 0.7 ps at code 504, 


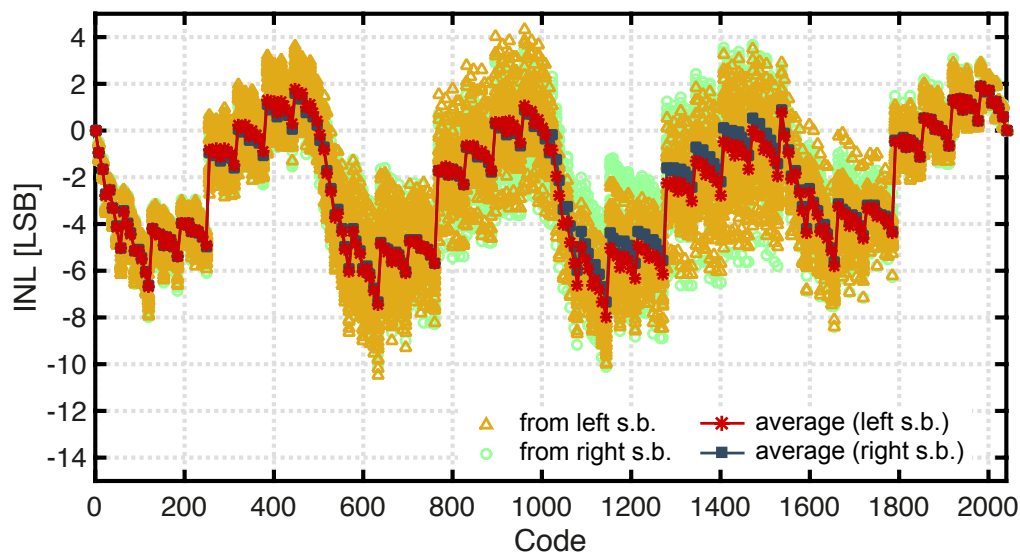

(a) side $R$

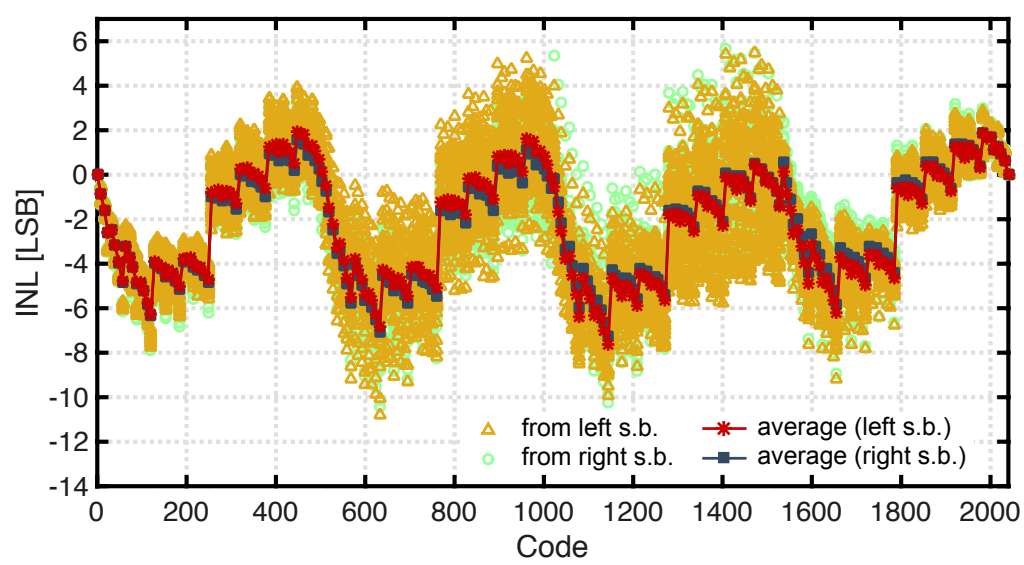

(b) side $F$

Figure 5.32: Measured INL of the combination coarse+fine DTC in UTDUCK.

corresponding to 1.4 LSB.

Fig. 5.32 shows the INL of the entire DTC (coarse+fine). The INL plot shows jumps at the codes corresponding to a transition from fine to coarse delay mechanism, resulting after the calibration. The DNL measurements are shown in Fig. 5.33. The maximum DNL is 4.09 LSB, occurring at the midcode 256 of the 9 -bit DAC in each fine-DTC. As expected, the DNL reflects exactly the profile of the delays in Fig. 5.29, since the DNL is obtained by subtracting from the measured delay steps their average and by expressing the result in LSB. 


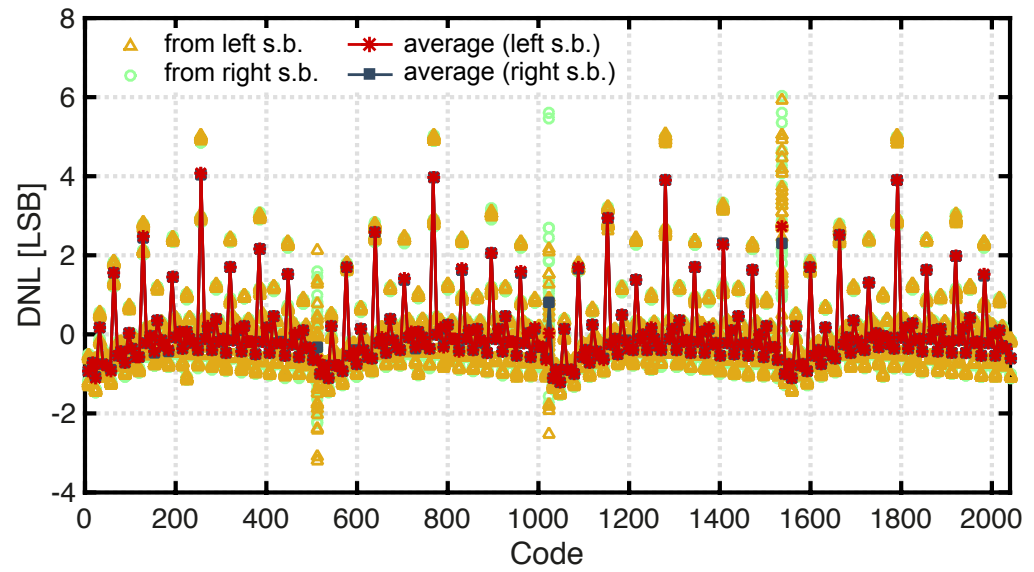

(a) side $R$

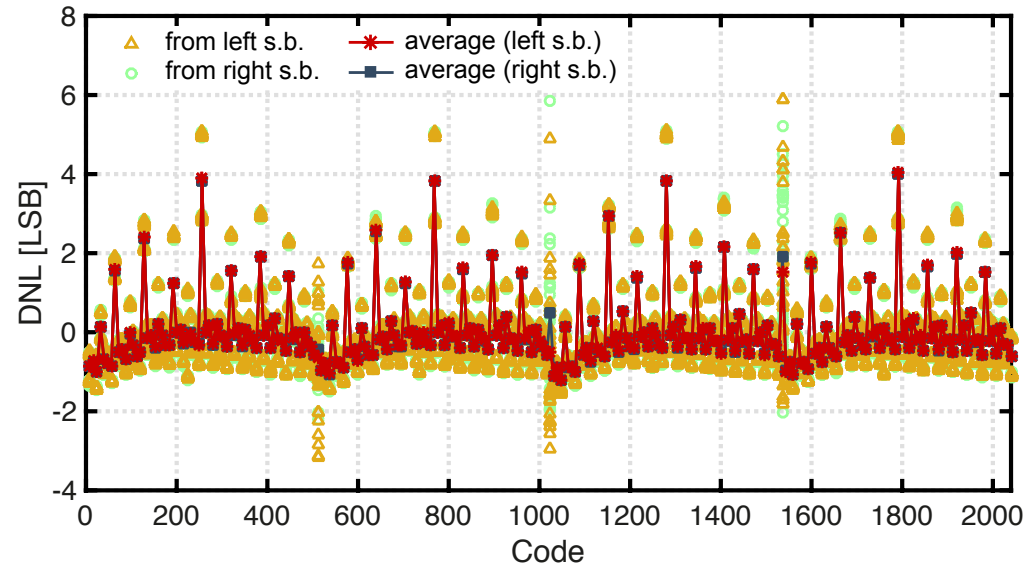

(b) side $F$

Figure 5.33: Measured DNL of the combination coarse+fine DTC in UTDUCK. 


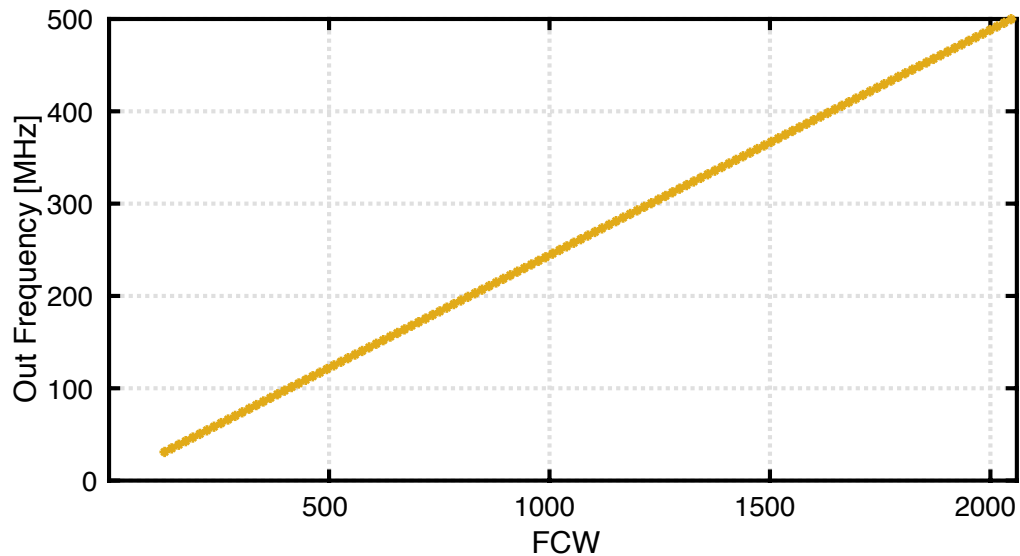

Figure 5.34: Measured output frequency of the DFC in the prototype as a function of the input word FCW. Measurements with $f_{\text {ref }}=2 \mathrm{GHz}$.

\subsubsection{Measurements in DFC mode}

The first measurement that can be done on the prototype in DFC mode is to check whether it synthesizes the correct frequencies. This can be seen in Fig. 5.34. With a fixed input reference frequency $f_{\text {ref }}=2 \mathrm{GHz}$, the input frequency word FCW is varied between 127 and 2048. Below 127 the output frequency is too low for the pass-band of the balun inserted between the chip and the spectrum analyzer. The output frequencies in Fig. 5.34 are as expected, with a linear control. This means that the digital part of the prototype is functionally correct.

When the circuit is in measurement mode with fixed DW $=0$ at the DTC input, it is possible to obtain the spectrum of the pulse-output DFC without DTC correction. This is shown in Fig. 5.35a for FCW $=1792$, which has the same profile as the simulated counter's MSB in Fig. 4.13. By switching the circuit to DFC-mode the effect of the DTC correction can be observed (Fig. 5.35b).

Fig. 5.36 shows the measured output spectrum of UTDUCK for FCW $=$ 780 together with the simulated spectrum from the behavioral model discussed in chapter 4, with an INL shape deduced from Fig. 5.32. More specifically, the average INL for the right sideband in Fig. 5.32a has been included in the behavioral model for the simulation. Other non-idealities inserted in the model are listed in the caption of Fig. 5.36 and they are motivated as follows. The value of the full-scale mismatch has been chosen to fit the measured mismatch spur in Fig. 5.36 at $f / f_{D D S}=0.667$. The errors in the clock phases are taken from the measured spur uncertainty in Fig. 5.29 for codes 512, 1024, 1536 which correspond to coarse-DTC transitions. The DTC fine full-scale error is chosen equal to the difference between the expected delay and the average delays in Fig. 5.29b and Fig. 5.29d. The spectrum in Fig. 5.36 obtained with the behavioral model shows a close fit 


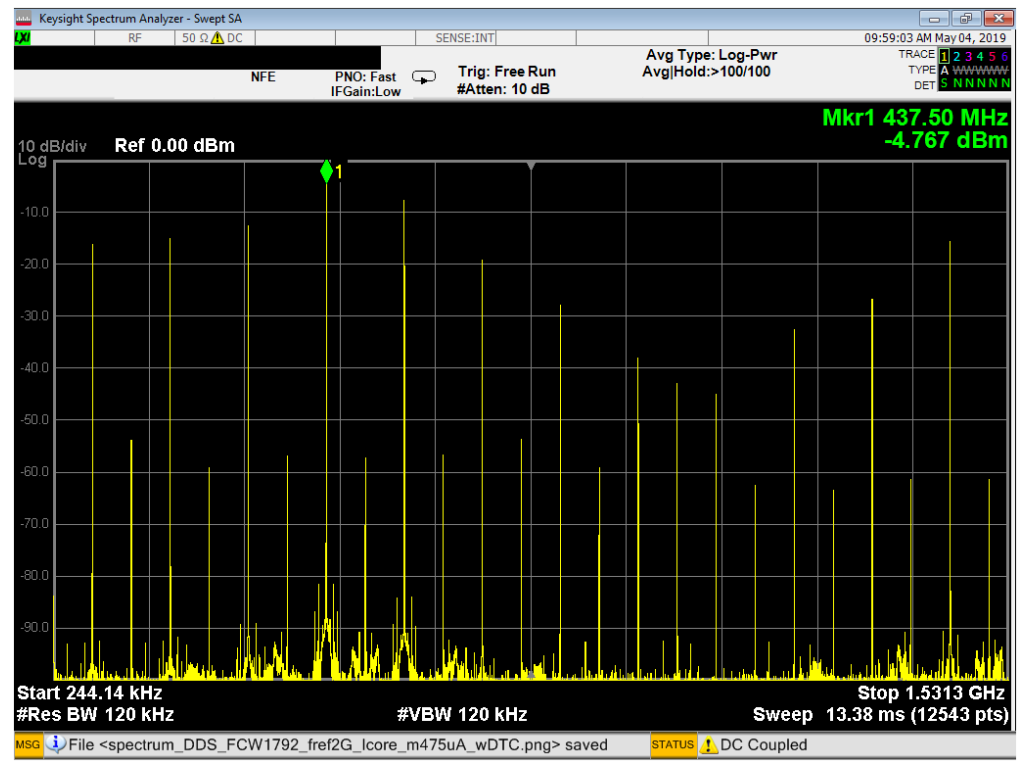

(a) without DTC

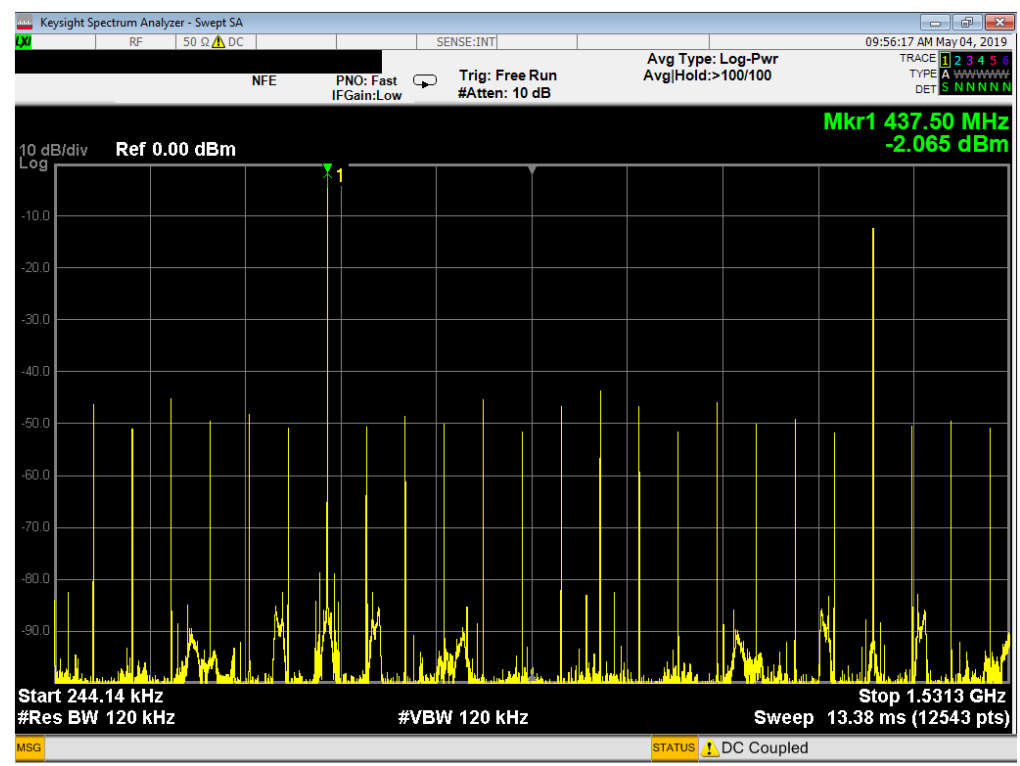

(b) with DTC correction

Figure 5.35: Measured output spectrum for $\mathrm{f}_{\text {ref }}=2 \mathrm{GHz}$ and FCW $=1792$, corresponding to an output frequency $\mathrm{f}_{\mathrm{DDS}}=437.5 \mathrm{MHz}$ from (4.1).

to the spur profile of the measured spectrum.

However, by keeping the same parameters, the model does not fit the measured spectrum for other values of FCW. For example, the case $\mathrm{FCW}=1792$, already considered in the simulations in chapter 4 , is shown in Fig. 5.37a. The reasons for this discrepancy can be i) a time-variant INL 


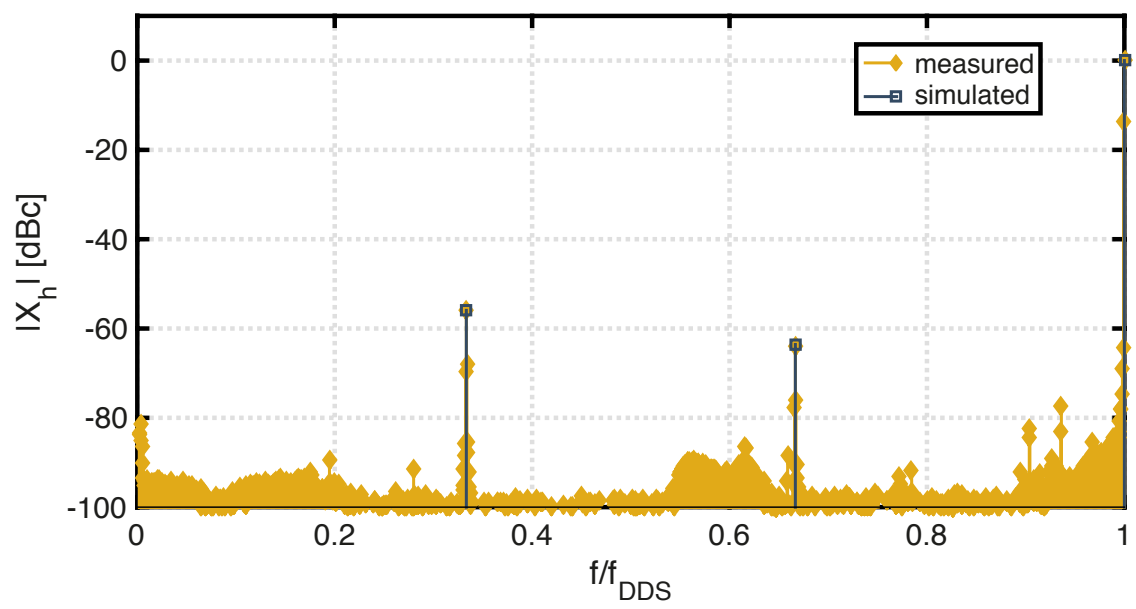

Figure 5.36: Measured output spectrum for FCW $=768$ and simulated spectrum from the behavioral model discussed in chapter 4, with the INL shape taken from Fig. 5.32 (average for the left sideband), error in the clock phases $\mathrm{CK}_{2}, \mathrm{CK}_{3}, \mathrm{CK}_{4}$ equal to $-3 \mathrm{LSB},+3 \mathrm{LSB}$ and $-3 \mathrm{LSB}$, respectively, 1 LSB error in the DTC fine full-scale and 12 LSB mismatch between the full-scales of the two interleaved fine-DTCs.

that differs from the average one in Fig. 5.32, ii) a resampling error in the fine-DW, as observed in the spur jumps of Fig. 5.30a. To prove that these reasons are plausible, we modeled both these errors for the fine-DW. More specifically, we used in the simulation the latest acquired INL pattern rather than the average INL in Fig. 5.32a and we modeled the code error with random jumps in the fine-DW. The resulting spectrum is shown in Fig. $5.37 \mathrm{~b}$, where the simulated sub-harmonic spurs in $\mathrm{f} / \mathrm{f}_{\mathrm{DDS}}=\{0.143,0.429,0.714\}$ approach the measured ones. Notice that by introducing the switching of DW between two possible values, the symmetry of the DW sequences is broken and this alters the spurs located at $f / f_{D D S}=\{0.286,0.571,0.857\}$. Thus, the difference between calculated and simulated spurs at these frequencies may depend on a different error pattern added to the nominal values of DW.

\subsubsection{Maximum spur}

Fig. 5.38 shows the measured maximum spur, in $\mathrm{dBc}$, for odd values of FCW (so that $\operatorname{GCD}\left(2^{\mathrm{N}}, \mathrm{FCW}\right)=1$ ), ranging from 127 to 2047 and the simulated maximum spurs, using the behavioral model discussed in chapter 4, with the same impairment values considered in Fig. 5.37b. The goal is to compare the measured results with the expected $6 \mathrm{~dB} /$ octave trend, as discussed in chapter 4 . The simulated curve shows expected $6 \mathrm{~dB} /$ octave slope, representing the trend of the measured points. However, we observe recurring bumps in the measured maximum spurs in Fig. 5.38. The highest measured spur is $-29.7 \mathrm{dBc}$ and corresponds to a bump peak at 


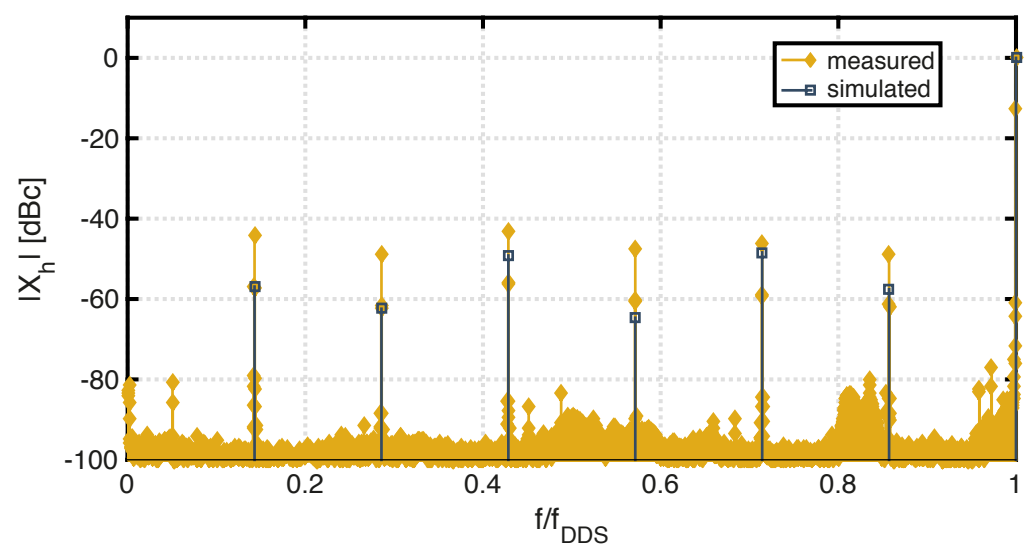

(a) FCW $=$ 1792. Same errors and INL as in Fig. 5.36

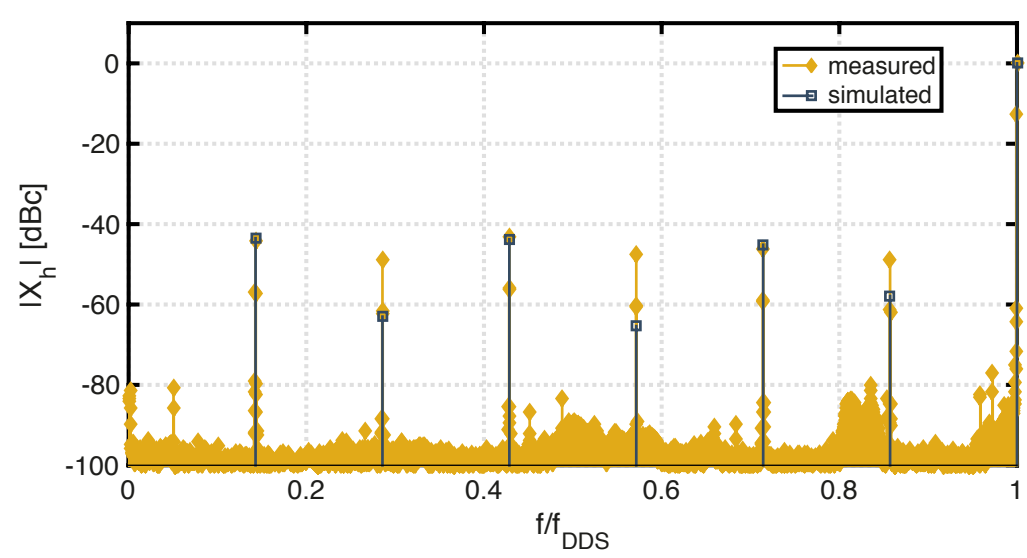

(b) Same as Fig. 5.37a, with added code errors in the model.

Figure 5.37: Measured and simulated output spectrum for FCW $=1792$.

$\mathrm{FCW}=1839$ and $\mathrm{f}_{\mathrm{DDS}}=449 \mathrm{MHz}$. The other distinguishable bump peaks are located at $\mathrm{FCW}=863,959,1087,1263,1487,1839$ and the corresponding maximum spurs are also aligned with a $6 \mathrm{~dB}$ /octave slope. The minima are at $F C W=815,911,1023,1167,1359,1375,1631,1647,2047$ and corresponds to spurs from $-51 \mathrm{dBc}$ to $-44.8 \mathrm{dBc}$, again with a slope equal to $6 \mathrm{~dB} /$ octave. At the highest output frequency $\left(f_{\mathrm{DDS}}=499.7 \mathrm{MHz}, \mathrm{FCW}=2047\right)$ the maximum spur is $-44.8 \mathrm{dBc}$. The reason for these bumps is still unknown and its study and effect on timing is a future recommendation.

\subsubsection{Phase noise measurements}

The measured phase noise at the output of the prototype (DFC mode) is shown in Fig. 5.39, for two different values of FCW: 2048 and 1792. The reference clock source is at $f_{\text {ref }}=2 \mathrm{GHz}$ and is provided by Rohde\&Schwarz SMP02 $10 \mathrm{MHz}-20 \mathrm{GHz}$ Microwave Signal Generator. FCW $=2048$ is the highest value possible, but it is a power of 2 , so the DTC always pro- 


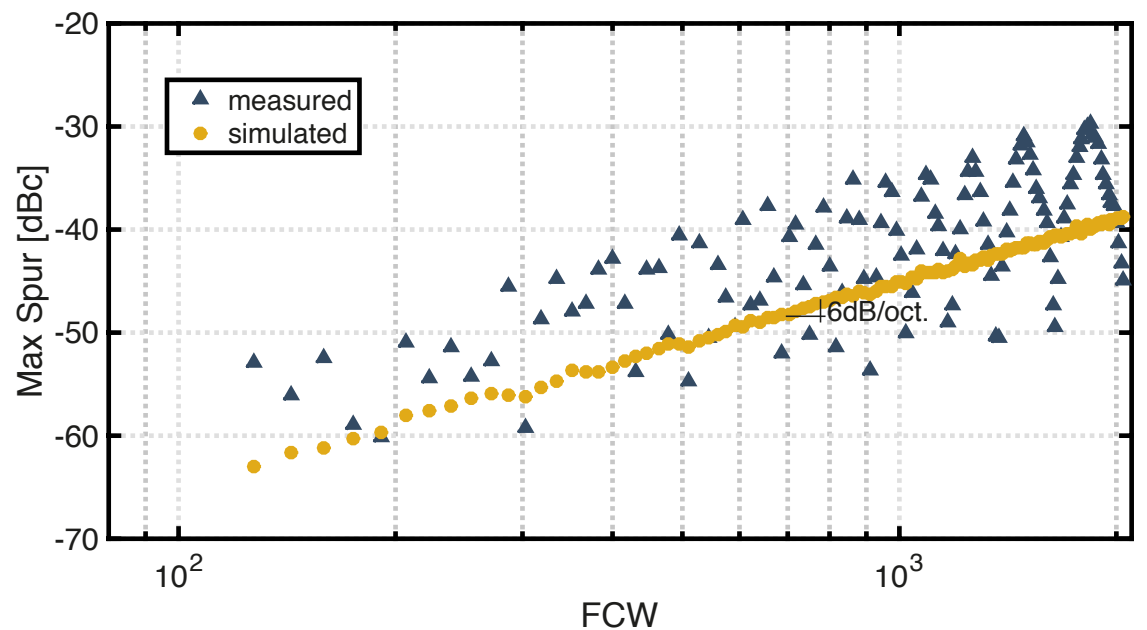

Figure 5.38: Measured maximum spur, in $\mathrm{dBc}$, for odd FCW ranging from 127 to 2047 with step-size 16 and simulated maximum spur. Simulations obtained with the behavioral model discussed in chapter 4, with the same impairment values considered in Fig. 5.37b.

vides a fixed delay. Instead FCW $=1792$ is an example of frequency word where the DTC changes the current delay word at almost every clock cycle. A marker table with the resulting integrated jitter is also shown in each figure. For Fig. 5.39a and 5.39b, the first row in each table shows the integrated jitter from $1 \mathrm{MHz}$ to $\mathrm{f}_{\text {carrier }} / 2$, the second marker refers to the band $1 \mathrm{kHz}-1 \mathrm{MHz}$, while the third marker considers the band $1 \mathrm{MHz}-10 \mathrm{MHz}$.

The DFC shows white frequency noise in the band $1 \mathrm{kHz}-100 \mathrm{kHz}$ (slope of $-20 \mathrm{~dB} /$ decade) [115], a bump in the phase noise around the frequency $\mathrm{f}_{\mathrm{CK}} / 2^{12}=244 \mathrm{kHz}$, corresponding to the minimum overflow rate of the counter, and a jitter in the band $1 \mathrm{MHz}-\mathrm{f}_{\text {carrier }} / 2$ in the order of $600 \mathrm{fs}$. By subtracting (in power) the jitter $317 \mathrm{fs}$ measured from the reference clock (equation (5.5)), the net contribution of the system is $509 \mathrm{fs}$ that is higher than the expected $363 \mathrm{fs}$ in section 5.5.2. This is plausibly due to a degradation of the output slope in (5.6). Thus, we can conclude that the measured jitter is $40 \%$ higher than the expected one, likely due to the parasitic capacitances that reduce the effective ramp slope.

The case FCW $=2048$ (Fig. 5.39a) presents less spurs than the one with FCW $=1792$ (Fig. 5.39b). This is consistent with expectations. In fact, FCW $=2048$ is a power of 2 , thus the counter already produces a square-wave with the target instantaneous frequency and the DTC always adds the same delay. In the case FCW $=1792$, instead, the DTC corrects the instantaneous frequency with a DW that changes periodically and multiple spurs appear in the measured phase noise. 


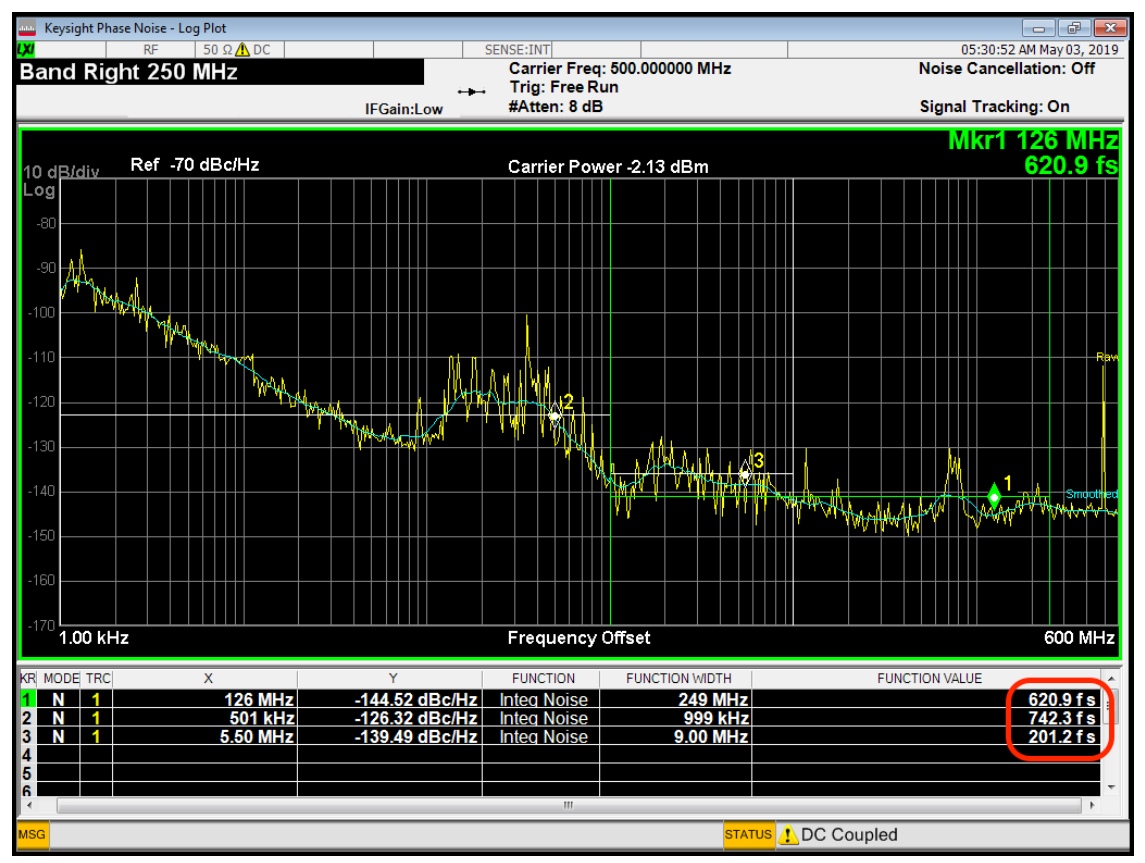

(a) $\mathrm{FCW}=2048$ (carrier at $500 \mathrm{MHz}$ )

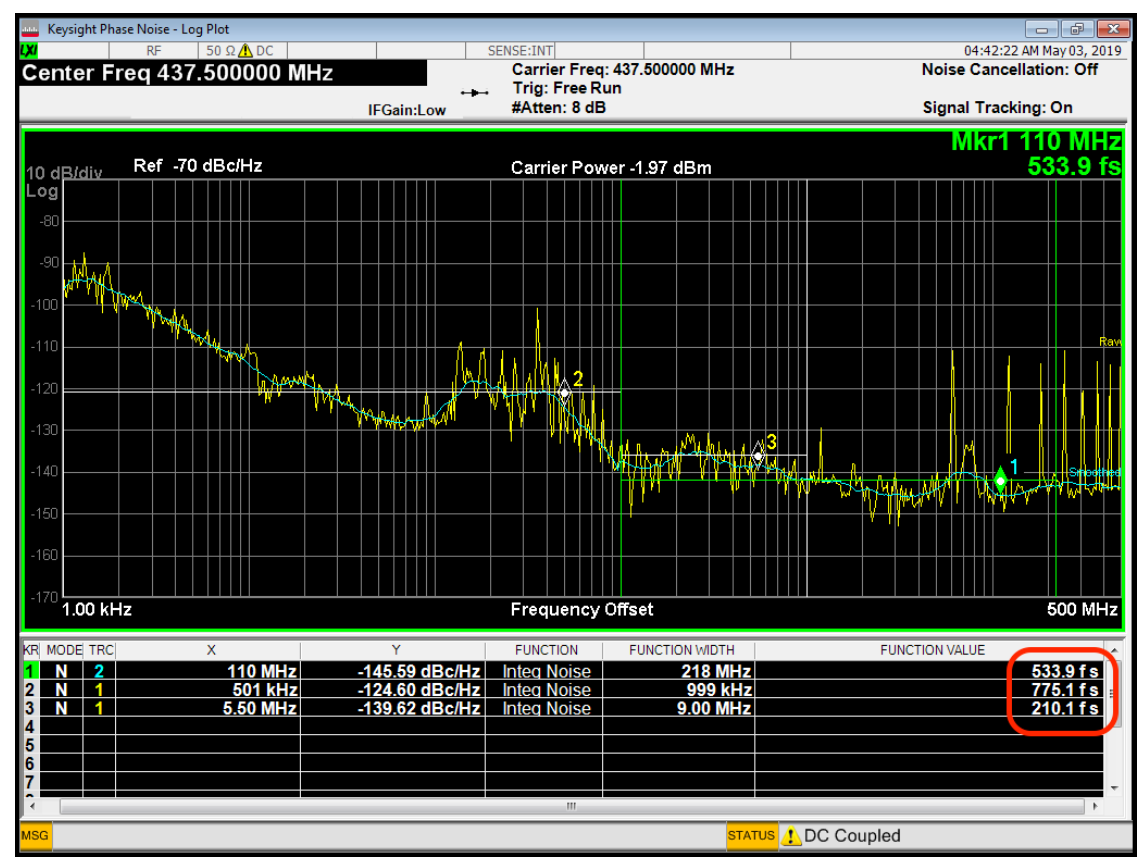

(b) $\mathrm{FCW}=1792$ (carrier at $437.5 \mathrm{MHz}$ )

Figure 5.39: Measured phase noise of the generator and the DFC. 


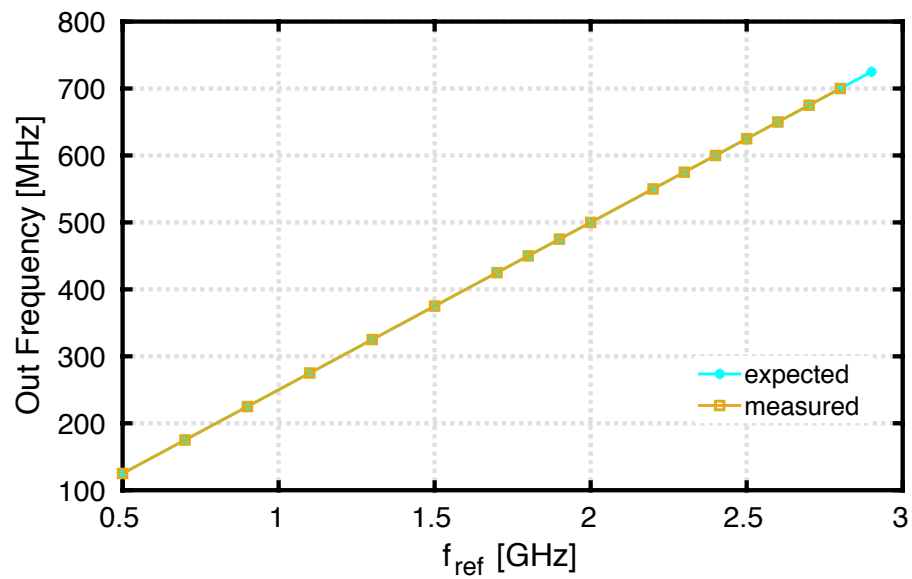

Figure 5.40: Speed test of the DFC. Output frequency as a function of $f_{\text {ref }}$, with fixed FCW $=2048$ and $\mathrm{I}_{\text {ref }_{\text {core }}}=475 \mu \mathrm{A}$.

\subsubsection{Speed measurements}

We verified the maximum speed of the DTC alone and the maximum speed of the entire system (DFC+DTC).

As for the entire system, we manually increased the reference frequency $f_{\text {ref }}$ until the resulting output spectrum showed the expected output frequency $f_{D D S}$. Fig. 5.40 shows the results, where the measured output frequency corresponds to the expected one until $f_{\text {ref }}=2.8 \mathrm{GHz}$, after which there is a flat output and the spectrum analyzer shows only its noise floor. Thus, we found $f_{\text {ref }_{\max }}=2.8 \mathrm{GHz}$ to be the maximum reference frequency for the prototype to correctly synthesize all the frequencies in the allowed FCW range $1-2048$. The maximum output frequency is therefore $f_{\mathrm{DDS}_{\text {max }}}=700 \mathrm{MHz}$. The expected value calculated in section 5.5 .1 was $f_{\text {ref }_{\max }}=3.6 \mathrm{GHz}$. This denotes a $22 \%$ increase of the delays in the critical path. Fig. 5.41 shows the output spectrum with $\mathrm{f}_{\text {ref }}$ max $=2.8 \mathrm{GHz}$ and $F C W=2047\left(f_{D D S}=699.66 \mathrm{MHz}\right)$. Higher reference frequencies produced the expected output frequency only for a reduced range of FCW.

When changing $f_{\text {ref, }}$, the fine-DTC full-scale should be re-calibrated by adjusting the core reference current $\mathrm{I}_{\text {ref }_{\text {core }}}$. Therefore, the core current should be increased from the value $475 \mu \mathrm{A}$ valid for $f_{\text {ref }}=2 \mathrm{GHz}$, to $\mathrm{I}_{\text {ref }_{\text {core }}}=780 \mu \mathrm{A}$ for $\mathrm{f}_{\text {ref }_{\max }}=2.8 \mathrm{GHz}$. See appendix A for further details.

As for the DTC speed limit, the characterization has been done with the prototype in measurement mode and with the phase modulation method (chapter 3). The frequency control word FCW determines the carrier frequency and it is set to the maximum value FCW $=2048$, while the DTC delay word DW is switched between 0 and 511 to periodically produce the maximum fine delay step. The path through an additional divider by 2 is selected to observe a single fine-DTC. Referring to equation (5.16), with the reference core current $\left(I_{\text {ref }_{\text {core }}}\right.$ in Fig. 5.8) kept constant and $f_{\text {ref }}$ 


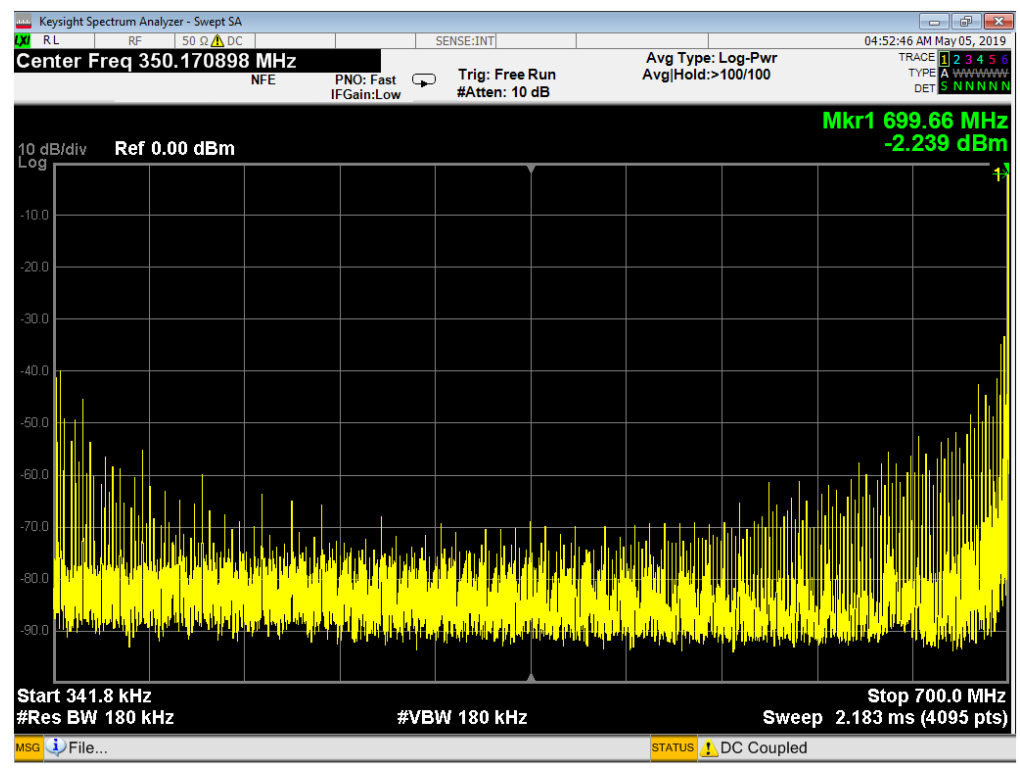

Figure 5.41: Measured output spectrum of the prototype with $f_{\text {ref }}=$ $2.8 \mathrm{GHz}$ and FCW $=2047\left(\mathrm{f}_{\mathrm{DDS}}=699.66 \mathrm{MHz}\right) . \mathrm{I}_{\text {ref }_{\text {core }}}=780 \mu \mathrm{A}$, while the other calibration parameters are kept the same. (See appendix A for further details.).

increasing, the delay step $\tau$ in (5.16) stays constant, while the carrier period $\mathrm{T}_{\text {carrier }}$ decreases, so the sideband-spurs are expected to increase. The DTC works properly as long as the sideband-spurs have the expected strength according to (5.16). When $f_{r e f}>f_{r e f}$ max the DTC output edge will not be determined anymore by DW, but instead by the arrival of next clock's edge, which scales with $f_{\text {ref }}$. Therefore, the produced delay step will be less than the expected value.

This is shown graphically in Fig. 5.42, where the inputs and the differential output of the ZCD in the fine-DTC core, are displayed over time, for two different values of $f_{r e f}$. In Fig. 5.42a, the ramps' crossing triggers the switching of the ZCD output and then the reset state, that appears in Fig. 5.42a with $Z C D_{i n N}$ and $Z C D_{i n P}$ going back to the rail values. Then the next clock's edge (actually MRE edge, not shown in Fig. 5.42a) concludes the reset state and a new DAC conversion starts, with the ZCD inputs connected to the interleaved fine-DTC. DW is kept to 511 in these simulations and determines the starting level of the ramps (highlighted in Fig. 5.42a with a dashed line). When $f_{\text {ref }}$ increases, the reset time where the ZCD inputs are at the rail levels decreases. When $f_{\text {ref }}>f_{\text {ref }}$ max , the situation is depicted in Fig. 5.42b: the MRE edge occurs already at the ramps' crossing, so the ZCD inputs do not have time to go to the rail levels, but instead they are directly connected to the other interleaved fine-DTC. This corrupts the starting level of the ramps and consequently the crossing 


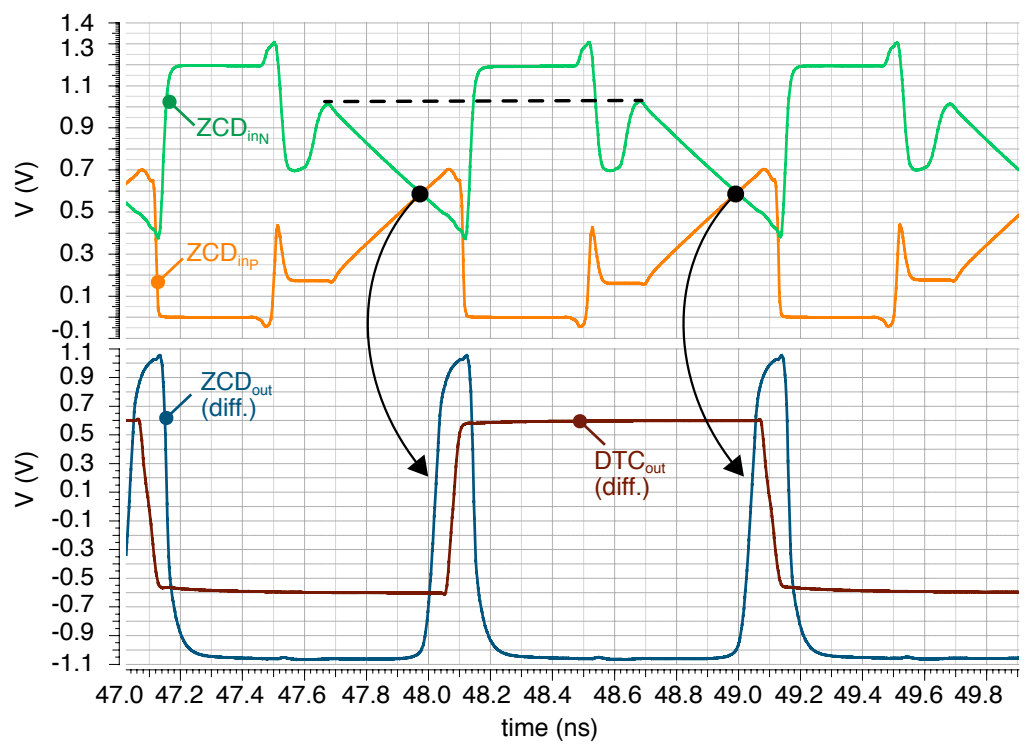

(a) $\mathrm{f}_{\text {ref }}<\mathrm{f}_{\text {ref }}$ max (Used in simulation: $\mathrm{f}_{\text {ref }}=2 \mathrm{GHz}, \mathrm{DW}=511$.)

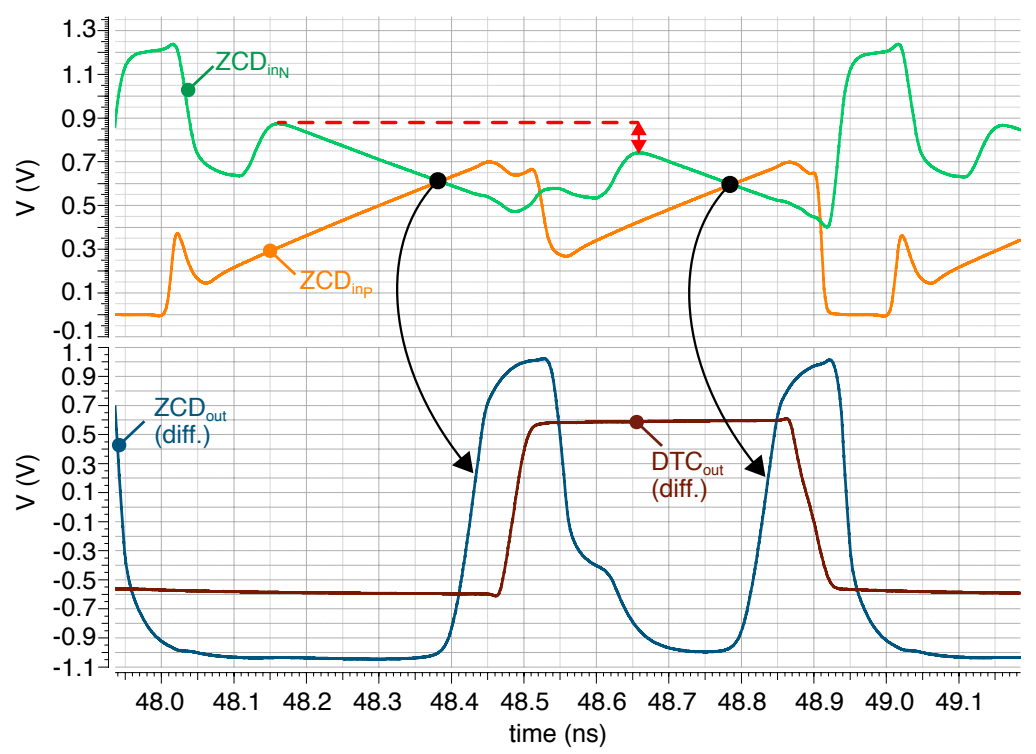

(b) $f_{r e f}>f_{\text {ref }_{\max }}$. (Used in simulation: $\mathrm{f}_{\text {ref }}=4 \mathrm{GHz}, \mathrm{DW}=511$.)

Figure 5.42: Internal waveforms resulting from simulation for two different values of $f_{\text {ref }}$.

time, that happens earlier. Thus the corresponding output edge is delayed less than the expected value for DW $=511$. In the case of Fig. 5.42b, the output still manages to switch and the unbalancing of the ZCD inputs still happens on one side. However, a small further increase of $f_{\text {ref }}$ will remove completely the ZCD input unbalancing and will corrupt more the starting level of the ramps, producing a flat output. 

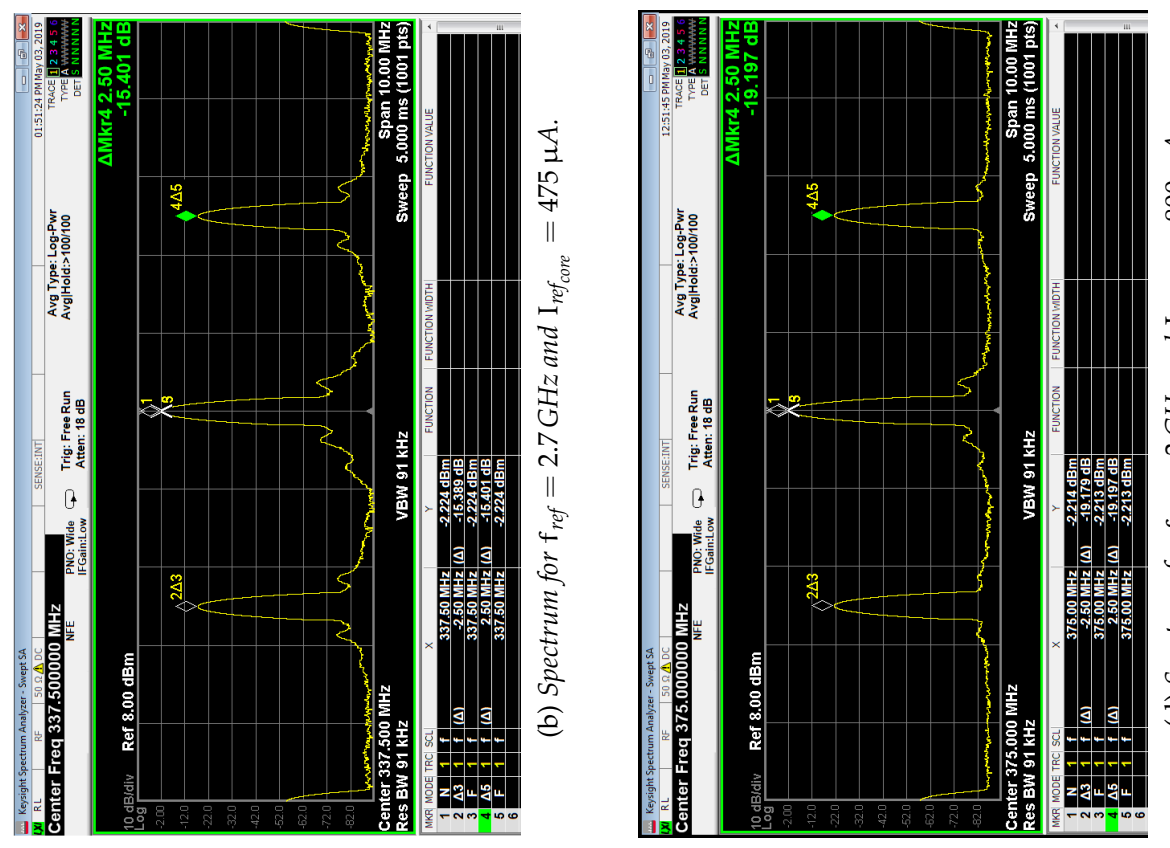

115
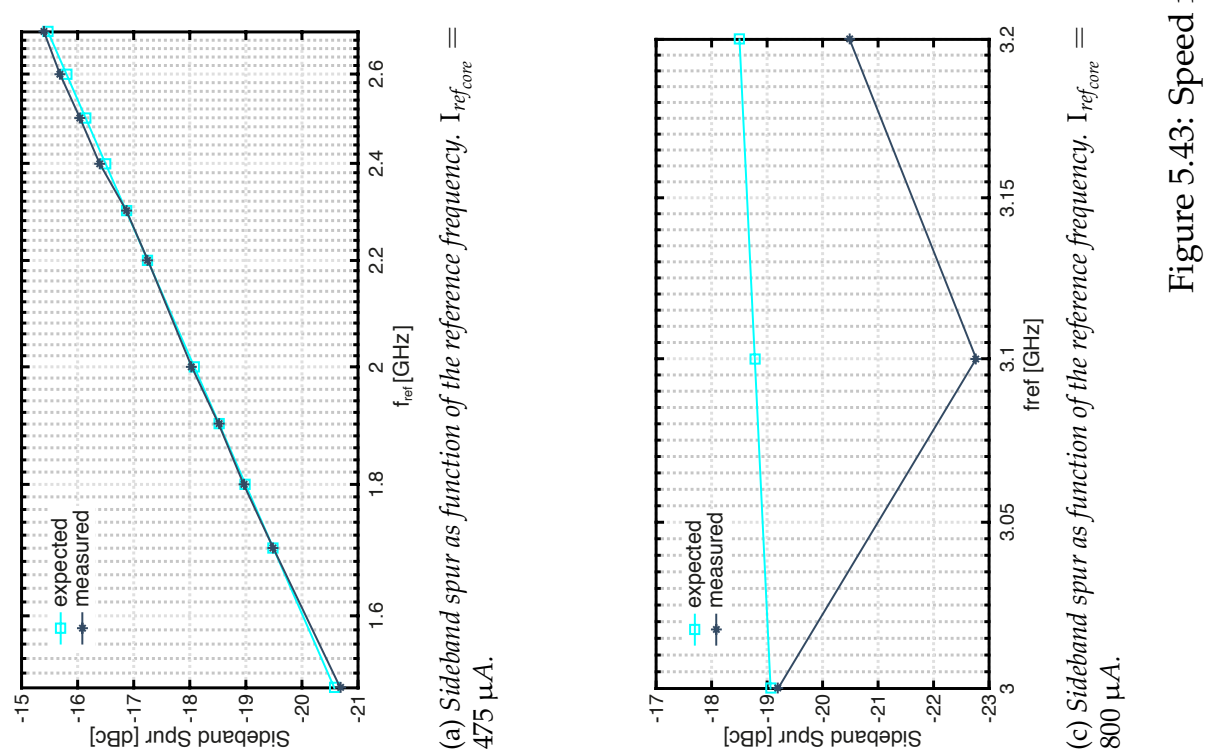
The DTC speed measurements has been done manually, by saving the spectrum and the marker table of the phase modulated output for each value of $f_{\text {ref }}$. The maximum DTC speed is reached for the value $f_{\text {ref }}$ max where the measured modulated spurs still corresponds to the expected one. For $f_{\text {ref }}>f_{\text {ref }} f_{\max }$, the DTC does not produce the expected delay for a given code, so the measured modulated spurs will deviate from expectations. Every measured spectrum is averaged over $>100$ traces, thus improving the measurement accuracy to $0.001 \mathrm{~dB}$ and keeping the sensitivity of the measurement method below 1 LSB (from (3.7) in chapter 3).

Fig. 5.43a shows the expected and the measured sideband spur when the current is fixed to $I_{\text {ref }_{\text {core }}}=475 \mu \mathrm{A}$. The values match until $\mathrm{f}_{\text {ref }}=$ $2.7 \mathrm{GHz}$, after which there's no output, due to the high delay-step produced compared to the reference frequency. Fig. 5.43b shows the spectrum for $f_{\text {ref }}=2.7 \mathrm{GHz}$, corresponding to the last point in Fig. 5.43a. The input signal delayed on both edges by the DTC has frequency $f_{\text {ref }} / 4=675 \mathrm{MHz}$. Then the extra divider makes the output carrier frequency $f_{\text {carrier }}=337.5 \mathrm{MHz}$ (marker 1 in Fig. 5.43b). By increasing the current to $\mathrm{I}_{\text {ref }}$ core $=800 \mu \mathrm{A}$, the DTC works properly at $f_{\text {ref }}=3 \mathrm{GHz}$, as shown in Fig. $5.43 \mathrm{c}$ and Fig. 5.43d. However for $f_{\text {ref }}>3 \mathrm{GHz}$ the measured sideband deviates from the expected value (Fig. 5.43c), denoting a situation similar to the case in Fig. 5.42b. Further increase of the reference core current does not produce the expected delay steps for $f_{\text {ref }}>3 \mathrm{GHz}$. The same results are obtained by selecting the other fine-DTC. Thus we can conclude that $\mathrm{f}_{\text {ref }} \mathrm{f}_{\max }=3 \mathrm{GHz}$ and the DTC is capable to delay both edges of a $3 \mathrm{GHz} / 4=750 \mathrm{MHz}$ input signal.

Since the maximum speed results are similar for both the cases of

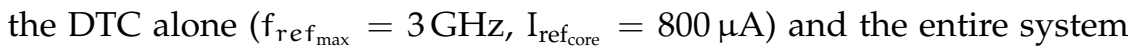
$\left(f_{\text {ref }_{\text {max }}}=2.8 \mathrm{GHz}, \mathrm{I}_{\text {ref }_{\text {core }}}=780 \mu \mathrm{A}\right)$, we can conclude that the speed bottleneck in the system is the DTC, particularly the succession of digital states, as it was estimated in section 5.5.1. The measured $f_{\text {ref }}$ max 2 is $22 \%$ less than the estimated value $3.6 \mathrm{GHz}$ (equation (5.4)).

\subsubsection{Power breakdown}

As for the power consumption, the power breakdown of the prototype UTDUCK in DFC mode for $f_{\text {ref }}=2 \mathrm{GHz}$ and FCW $=2047$ (output frequency 499.7 MHz) is shown in Fig. 5.44. The prototype has different supply voltages: $V_{D_{A_{A}}}$ provides the supply of the DTC core (current generators and $\mathrm{ZCD}$ ). It is set at $1.2 \mathrm{~V}$ and consumes $12.5 \mathrm{~mA}$. $\mathrm{V}_{\mathrm{DD}_{\mathrm{Z}}}$ unbalances the $\mathrm{ZCD}$ (through the switches $R S T_{Z}$ ), dissipating mostly dynamic power. It is set at $1.2 \mathrm{~V}$ and consumes $0.7 \mathrm{~mA}$. All the sources of dynamic power are measured at the aforementioned values of $\mathrm{f}_{\text {ref }}$ and FCW. The DAC reference voltages are set to $1.240 \mathrm{~V}$ and $1.249 \mathrm{~V}$ to compensate for capacitance-mismatches between the two sides $\mathrm{R}-\mathrm{F}$ and they both consume $3.3 \mathrm{~mA}$. Other sources of mostly dynamic power are the digital supplies $V_{D_{D} D_{D S w}}$ and $V_{D_{D}}$. The 


\begin{tabular}{|c|c|c|c|}
\hline $\begin{array}{c}\text { Supply } \\
\text { name }\end{array}$ & Voltage $[\mathrm{V}]$ & Current $[\mathrm{mA}]$ & Power $[\mathrm{mW}]$ \\
\hline $\mathrm{V}_{\mathrm{DD}_{\mathrm{A}}}$ & 1.2 & 12.5 & 15.0 \\
\hline $\mathrm{V}_{\mathrm{DD}_{\mathrm{Z}}}$ & 1.2 & 0.7 & 0.8 \\
\hline $\mathrm{V}_{\mathrm{REF}_{\mathrm{DAC}-\mathrm{R}}}$ & 1.240 & 3.3 & 4.1 \\
\hline $\mathrm{V}_{\mathrm{REF}_{\mathrm{DAC}-\mathrm{F}}}$ & 1.249 & 3.3 & 4.1 \\
\hline $\mathrm{V}_{\mathrm{DD}_{\mathrm{DSW}}}$ & 0.9 & 5.1 & 4.6 \\
\hline $\mathrm{V}_{\mathrm{DD}_{\mathrm{D}}}$ & 1.2 & 18.1 & 22.3 \\
\hline $\mathrm{V}_{\mathrm{DD}_{\mathrm{OB}}}$ & 1.2 & 18.7 & 22.4 \\
\hline
\end{tabular}

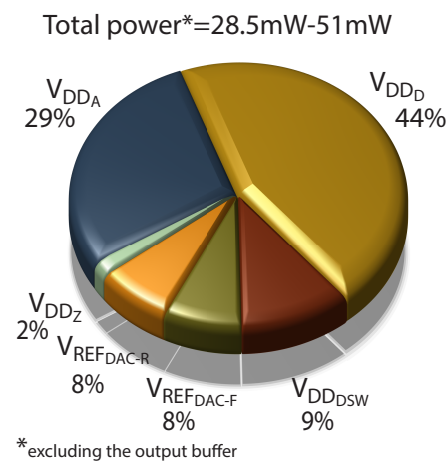

Notes: Measured values in table with the prototype in DFC mode, at $f_{\text {ref }}=2 \mathrm{GHz}$ and output frequency $499.7 \mathrm{MHz}$ (corresponding to $\mathrm{FCW}=2047$ ). Total power evaluated for the entire range $\mathrm{FCW}=1-2048$.

Figure 5.44: Power breakdown of the prototype UTDUCK. The pie chart represents the breakdown excluding the output buffers placed for measurement purposes.

former is connected to the digital block synthesized with automatic tools; it is set to $0.9 \mathrm{~V}$ consuming $5.1 \mathrm{~mA}$. The latter supplies the custom-digital blocks (both CMOS and CML). It is set to $1.2 \mathrm{~V}$ consuming $18.1 \mathrm{~mA}$. The output buffers driving the $50 \Omega$ are supplied by $V_{D_{D}}$ о that is set to $1.2 \mathrm{~V}$ and consumes $18.7 \mathrm{~mA}$. Therefore, the total power of the circuit, excluding the output buffers which are placed for measurement purposes, is $51 \mathrm{~mW}$. With the same $f_{\text {ref }}$, by varying the input control word FCW from 1 to 2048, the total output power goes from $28.5 \mathrm{~mW}$ to $51 \mathrm{~mW}$.

From the pie chart in Fig. 5.44, it can be observed that the biggest currents are drawn from the supplies $V_{D_{D}}$ and $V_{D_{D}}$. Fig. 5.45 shows how the power is split among the different modules connected to these supplies. The four-phase clock requires on average the highest current from $V_{D_{D}}$, thus being responsible for $46 \%$ of its power consumption. However, in the context of Systems-on-Chip (SoCs), the four-phases can be shared with other blocks so the power required to the four-phase generator has less impact on the DFC power budget. The data buffers, which require $13 \%$ of the $V_{D_{D}}$ total power, have been introduced in the prototype at the interface between the digital block synthesized with automatic tools and the custom DAC designed manually. However they can be reduced or removed with a better control of the boundary conditions in the digital design flow, implying a recursive design based on an accurate parasitic extraction of the custom circuit. Finally the coarse DTC can be made more power efficient by switching off the resamplig flip-flops that are not going to be selected, at the expense of circuit complexity. As for the power breakdown of $V_{D_{D}}$ in Fig. 5.45, half of the total current consumption is due to the DTC core, that requires a constant current. 


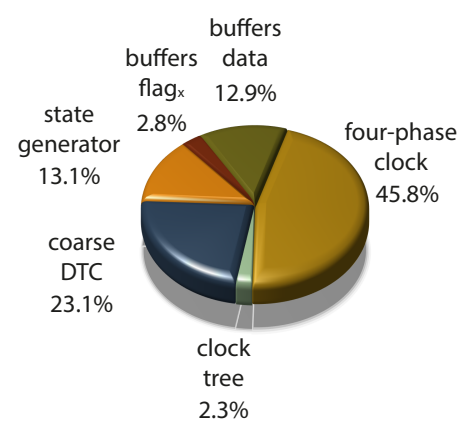

(a) $V_{D_{D}}$

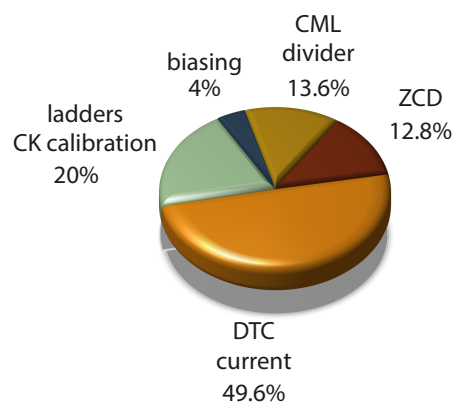

(b) $\mathrm{V}_{\mathrm{DD}}$

Figure 5.45: Simulated power breakdown for the supplies $V_{D_{D}}$ and $\mathrm{V}_{\mathrm{DD}_{\mathrm{A}}}$.

\subsection{Benchmarking}

Table 5.3 shows recent published DTCs, as well as the performances of the DTC in the prototype UTDUCK. The proposed DTC offers an independent control of both the edges of the carrier, but it suffers from a sub-optimal power consumption of the analog supply $V_{D_{D}}$, so thus increasing the final Figure-of-Merit (FoM).

Table 5.4 compares UTDUCK with a number of published DTC-based frequency synthesizers, both Digital-to-Period Converters (DPCs) and DFCs. The measured SFDR of the prototype is comparable to the work [42] but without the usage of additional orthogonal techniques, such as dithering or $\Sigma \Delta$ modulators to modify the instantaneous FCW. The bumps observed in Fig. 5.38 seriously worsen the best achievable spur by $10 \mathrm{~dB}$. As for the power consumption, the current prototype is not competitive with the state-of-art digital synthesizers.

Usually the FoM of a digital synthesizer is a function of the Effective Number of Bits (ENOB) [116] that in turn is related to the Signal-to-Noise And Distortion ratio (SINAD) [73]. However, being the output waveform a square-wave (so distortion is intended), it would be more significant to define a FoM in terms of Spurious-Free Dynamic Range (SFDR).

The minimum power consumed by a circuit clocked with frequency $f_{C K}$ is the dynamic power [26]:

$$
P=f_{C K} C_{\text {tot }} V_{D D}^{2}
$$

Where $V_{D D}$ is the circuit's power supply and $C_{\text {tot }}$ the circuit's total capacitance. Therefore it is:

$$
f_{C K} \propto P
$$

In chapter 4 we derived the closed form expression (4.31) which related the output SFDR of the digital synthesizer to its output frequency. We can 
Table 5.3: COMPARISON OF RECENT DTC IMPLEMENTATIONS.

\begin{tabular}{|c|c|c|c|c|c|c|c|}
\hline & $\begin{array}{c}\text { DTC in this } \\
\text { work }\end{array}$ & $\begin{array}{l}\mathrm{Ru}[\mathrm{CP}: 2] \\
\text { JSSC'15 }\end{array}$ & $\begin{array}{l}\text { Chen [56] } \\
\text { ASSCC '17 }\end{array}$ & $\begin{array}{l}\text { Markulic [50] } \\
\text { ESSCIRC '14 }\end{array}$ & $\begin{array}{c}\text { Sievert [57] } \\
\text { JSSC'16 }\end{array}$ & $\begin{array}{c}\text { Elmallah [61] } \\
\text { CICC'18 }\end{array}$ & $\begin{array}{c}\text { Elkholy [28] } \\
\text { JSSC'18 }\end{array}$ \\
\hline Technology & $65 \mathrm{~nm}$ & $65 \mathrm{~nm}$ & $28 \mathrm{~nm}$ & $28 \mathrm{~nm}$ & $28 \mathrm{~nm}$ & $65 \mathrm{~nm}$ & $65 \mathrm{~nm}$ \\
\hline Supply [V] & 1.2 & 1.2 & 1.0 & 0.9 & 1.1 & 1.05 & 0.9 \\
\hline $\begin{array}{c}\text { Active Area } \\
{\left[\mathrm{mm}^{2}\right]}\end{array}$ & 0.14 & 0.06 & 0.004 & 0.04 & 0.009 & 0.084 & 0.017 \\
\hline Architecture & const.-slope & const.-slope & const.-slope & variable-slope & $\begin{array}{c}\text { variable-slope } \\
\text { (PI) }\end{array}$ & variable-slope & variable-slope \\
\hline $\begin{array}{c}\text { Delay Range } \\
\text { [ps] }\end{array}$ & 1000 & 189 & 3.3 & 563 & 500 & 5310 & 350 \\
\hline $\begin{array}{l}\text { Resolution } \\
\text { [fs] }\end{array}$ & 488 & 185 & 103 & 550 & 244 & 330 & 2000 \\
\hline $\begin{array}{l}\text { Peak INL } \\
\text { [LSB] }\end{array}$ & 7.97 & 1.77 & 0.73 & 1.80 & 4.92 & 5.00 & 1.00 \\
\hline $\begin{array}{l}\text { Peak DNL } \\
\text { [LSB] }\end{array}$ & 4.09 & N/A & 0.35 & 1.44 & 1.25 & 3.03 & N/A \\
\hline $\begin{array}{c}\text { Dynamic } \\
\text { Range } \dagger \\
{[\mathrm{dB}]}\end{array}$ & 48.3 & 55.2 & 32.8 & 55.1 & 52.4 & 70.2 & 4.35 \\
\hline Jitter $\left[\mathrm{fs}_{\mathrm{rms}}\right]$ & 547 & 210 & 1209 & 250 & 244 & 340 & N/A \\
\hline $\begin{array}{c}\text { Frequency } \\
{[\mathrm{MHz}]}\end{array}$ & $1000^{*}$ & 55 & 40 & 40 & 2000 & 100 & 1000 \\
\hline Power [mW] & 15.0 & 1.8 & 0.015 & 0.5 & 19.8 & 10.13 & $<3$ \\
\hline $\begin{array}{l}\text { FoM } \ddagger \\
{[f J / c o n v]}\end{array}$ & 58.3 & 56.7 & 8.5 & 22.0 & 23.8 & 31.1 & $<17$ \\
\hline
\end{tabular}

Notes: $\nmid$ Dynamic range $=20 \log _{10}\left(\frac{\text { Delay Range }[\mathrm{s}]}{\mathrm{INL}[\mathrm{s}]}\right) ; \ddagger \mathrm{FoM}=\frac{\mathrm{P}}{\mathrm{f}_{\mathrm{DTC}}} \frac{\mathrm{INL}}{\text { Delay Range }}$, as in (2.2); * the DTC can control independently both the edges of the carrier, so the DTC frequency is double the carrier frequency.

re-write (4.31) using the definition of SDFR [73] and (4.1) as:

$$
10^{\frac{\mathrm{SFDR}[\mathrm{dB} c]}{20}}=\max _{h} \frac{\left|\mathrm{X}_{\mathrm{h}}\right|}{\mathrm{A} / \pi} \approx \frac{1}{2^{\mathrm{N}}}\left(1+\zeta \mathrm{INL}_{\max }\right) \frac{\mathrm{f}_{\mathrm{DDS}}}{\mathrm{f}_{\mathrm{CK}}}
$$

Thus:

$$
10^{\frac{S F D R[d B c]}{20}} \propto \frac{f_{D D S}}{f_{C K}}
$$

And, from (5.20), we can conclude that

$$
10^{\frac{\mathrm{SFDR}[\mathrm{dBc}]}{20}} \propto \frac{\mathrm{f}_{\mathrm{DDS}}}{\mathrm{P}}
$$

We can therefore define the following FoM for a digital synthesizer:

$$
\text { FoM }=\frac{f_{\text {DDS }}[\mathrm{GHz}]}{10^{\frac{\text { SFDR }[\mathrm{dBc}]}{20}} \times \text { Power }[\mathrm{mW}]}
$$

and use it to compare the performances of the state-of-the-art architectures, as shown in Table 5.4. The best FoM is achieved by [28] that is based on 
Table 5.4: PERFORMANCE COMPARISON WITH OTHER DTC-BASED SYNTHESIZERS

\begin{tabular}{|c|c|c|c|c|}
\hline $\begin{array}{l}\text { DTC-based } \\
\text { DDS Metrics }\end{array}$ & This work & $\begin{array}{c}\text { Talwalkar[42] } \\
\text { RFIC } 2010\end{array}$ & $\begin{array}{c}\text { Rapinoja [96] } \\
\text { TMTT } 2010\end{array}$ & $\begin{array}{c}\text { Elkholy [28] } \\
\text { JSSC } 2018\end{array}$ \\
\hline Technology & $65 \mathrm{~nm}$ & $90 \mathrm{~nm}$ & $65 \mathrm{~nm}$ & $65 \mathrm{~nm}$ \\
\hline DDS type & DFC & DPC & DPC & DFC \\
\hline $\begin{array}{c}\text { DTC } \\
\text { Resolution }\end{array}$ & $11 \mathrm{bit}$ & 5 bit & 4bit & 7bit \\
\hline $\begin{array}{l}\text { Freq. range } \\
{[\mathrm{MHz}]}\end{array}$ & $0.342-700$ & $2-1000$ & $100-4267$ & $20-1000$ \\
\hline $\begin{array}{l}\text { Frequency } \\
\text { Resolution }\end{array}$ & $324 \mathrm{kHz}$ & $\leq 15 \mathrm{~Hz}$ & $0.025-5.38 \mathrm{~Hz}$ & $125 \mathrm{MHz}$ \\
\hline $\begin{array}{l}\text { SFDR [dBc] } \\
\text { @ out. freq. }\end{array}$ & $\begin{array}{l}-29.7 @ 449.0 \mathrm{MHz} \\
-44.8 @ 499.7 \mathrm{MHz}\end{array}$ & $\begin{array}{l}-24.5 @ 997 \mathrm{MHz}^{(2)} \\
-48.2 @ 997 \mathrm{MHz}^{(3)}\end{array}$ & $-25 @ 528 \mathrm{MHz}^{(4)}$ & $-55 @ 500 M H z^{(6)}$ \\
\hline Power & $\begin{array}{c}51.0 \mathrm{~mW} \\
@ 500 \mathrm{MHz}^{(1)}\end{array}$ & $\begin{array}{l}\text { 146.4mW } \\
@ 997 \mathrm{MHz}\end{array}$ & $\begin{array}{c}8.4 \mathrm{~mW} \\
@ 4.267 \mathrm{GHz}\end{array}$ & $\begin{array}{l}3.2 \mathrm{~mW} \\
@ 1 \mathrm{GHz}\end{array}$ \\
\hline $\begin{array}{c}\text { FoM } \\
{[\mathrm{GHz} / \mathrm{mW}]}\end{array}$ & $\begin{array}{l}0.27 \\
1.70\end{array}$ & $\begin{array}{l}0.11^{(2)} \\
1.75^{(3)}\end{array}$ & 1.12 & 87.87 \\
\hline Notes & $\begin{array}{l}\text { (1) excluding } \\
\text { output buffers }\end{array}$ & $\begin{array}{l}{ }^{(2)} \text { no dithering } \\
{ }^{(3)} \text { with dithering }\end{array}$ & $\begin{array}{c}{ }^{(4)} \text { with } 2^{\text {nd }} \text { order } \\
\Sigma \Delta\end{array}$ & $\begin{array}{c}{ }^{(6)} \text { with } 1^{\text {st }} \text { order } \\
\Sigma \Delta\end{array}$ \\
\hline
\end{tabular}

dividers and a series of variable-slope CMOS DTCs, allowing to sensibly reduce the power consumption. Compared to [28], UTDUCK offers finer frequency resolution and an higher ratio $f_{D D S \max } / f_{D D S} \min$.

The performances of the prototype UTDUCK are affected by a suboptimal design and likely also errors occurring at the interface between analog and digital blocks. However the concept can still be competitive with the state-of-the-art. In fact, with impairments kept at $1 \mathrm{LSB}$, the achievable maximum spur can go below $-55 \mathrm{dBc}$, as shown in Fig. 5.46 (case B). Improvements on the design can lower the power consumption. If the four-phase clock can be re-used for other blocks in a SoC and its power contribution could be excluded from the power budget the total remaining power could be lowered to $40 \mathrm{~mW}$ that would bring the FoM of UTDUCK to $2.16 \mathrm{GHz} / \mathrm{mW}$.

To summarize, the current prototype needs further improvements to be competitive with the state-of-the-art, since it is limited by uncalibrated internal mismatches and time violations in the fine-DW sampling. However, there is margin for improvements to outperfom the state-of-the-art digital synthesizers. 


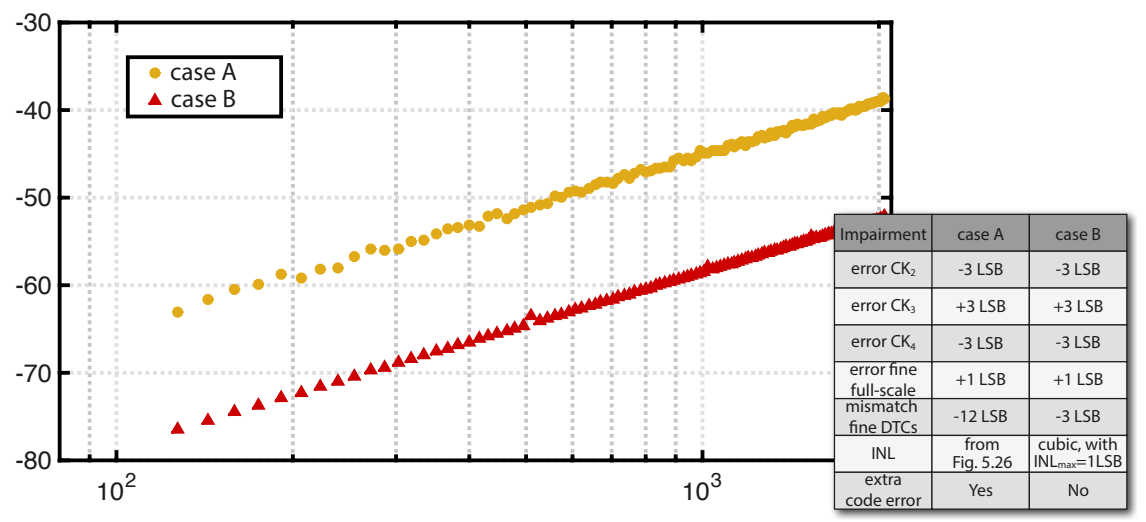

Figure 5.46: Comparison between simulated maximum spur as function of FCW. The two plots differ for the impairment values used, that are listed in the annexed table.

\subsection{Conclusions}

Direct Digital Synthesis is gaining renewed interest for its flexibility and compatibility with digital design flows. The recent research in this field sees DTCs as key blocks to sensibly improve the spectral performance of direct digital synthesizers, in both DFC and DPC form. The design of DTC-based frequency synthesizers faces various challenges mainly related to the integration of the digital core with the DTC (section 5.3.1).

Using a DTC at the output of a phase accumulator (pulse-output DFC) to correct the deterministic error associated with the accumulator's MSB is an alternative to other spur-correction techniques, like dithering or sigmadelta modulators (Table 5.4). The proposed DFC+DTC architecture relies on the deterministic correction of the spurious tones in the output spectrum (chapter 4), instantaneously and without converting them in noise floor (Fig. 5.35a and Fig. 5.35b). However, the effectiveness of the correction mechanism relies on the DTC resolution and linearity.

In this chapter, a prototype to verify the concept of spur-correction with a DTC has been discussed and its design in $65 \mathrm{~nm}$ CMOS technology has been described. It consists of a phase accumulator and a constant-slope high-speed DTC, with a coarse-fine scheme to produce delay (section 5.1). It operates at $1.2 \mathrm{~V}$, with a maximum input reference frequency of $2.8 \mathrm{GHz}$ (section 5.7.7) and an output frequency range $342 \mathrm{kHz}-700 \mathrm{MHz}$.

The prototype operates correctly at functional level, i.e. it produces the correct frequency for each input word FCW, but it needs further improvements to be competitive with the state-of-the-art, since it is limited by uncalibrated internal mismatches and time-violations in the fine-DW sampling. As a result, SFDR is rather poor and varies strongly with frequency, e.g. $-30 \mathrm{dBc}$ at $449 \mathrm{MHz}$ and $=-45 \mathrm{dBc}$ at $\mathrm{f}_{\mathrm{DDS}}=499.7 \mathrm{MHz}$, with a power consumption of $51 \mathrm{~mW}$ excluding the output buffers added for 
measurement purposes to drive the external $50 \Omega$ load.

With a wider calibration range and the possibility to calibrate mismatches between the R and F sides of the DTC, together with a more careful layout and a consequent improved power efficiency, the spur-reduction is expected to be much more, with $\mathrm{SFDR}=-66.6 \mathrm{dBc}$ (Fig. 5.46).

Overall, it can be concluded that the spur-reduction technique based on DTC correction is promising to realize flexible digitally programmable frequency synthesis. Further improvement in the output spectrum can be obtained by incorporating orthogonal techniques like dithering or $\Sigma \Delta$ modulation of the input word FCW with minimal extra power consumption, at the expense of the output noise floor. 


\section{CHAPTER}

\section{Conclusions \& Recommendations}

In this chapter, the work presented in this thesis is summarized and the key conclusions are drawn, followed by a presentation of the original contributions. Finally, recommendations for future work are discussed.

\subsection{Summary \& Conclusions}

Direct Digital Synthesis, being known from the 1970's, is now gaining renewed interest, especially in the context of Systems-on-Chip (SoCs), due to the technological advances that allow for low-power high-speed digital, as well as flexibility, immediate and phase-continuous frequency hopping, wide frequency range and extremely fine frequency resolution. These features suit well portable devices, which are boosting the electronics global market (chapter 1). A Digital-to-Time Converter (DTC) can be a key block to substantially improve the output spectrum of Digital-toFrequency Converters (DFCs) that produce square-waves, as an alternative or in conjunction with the other techniques mentioned in section 1.1, like dithering or $\Delta \Sigma$ modulation. The main objective of this thesis is to gain insight in the design trade-offs and performance limitations of CMOS architectures for digital frequency synthesis, producing square-wave clocks, with a DTC-based compensation mechanism to achieve low deterministic jitter.

We explored the feasibility of a low GHz Pulsed-Output DDS exploiting a DTC for deterministic jitter correction and targeting $<-60 \mathrm{dBc}$ spur level. This requires DTCs having GHz operating frequencies, sub-picosecond resolution and a Figure-of-Merit (FoM) of a few tens of fJ/conversion (section 2.3). Most of the best performance DTCs are to some extent based on the constant-slope principle [CP:2], consisting on a constant current source charging a capacitor with constant voltage slope, starting from a programmable initial voltage. This results in Integral Non-Linearity 
(INL) and Differential Nonlinearity (DNL) of a few LSBs, as the delay of the threshold crossing detector is constant as it always "sees" the same constant-slope voltage. (section 2.1). The different DTC architectures discussed in section 2.2 mainly differ in the generation of the starting capacitor voltage, to improve the DTC efficiency.

With DTCs able to provide sub-picosecond delay-steps, it becomes challenging to reliably measure such small delays and consequently characterize a DTC in terms of INL and DNL. In chapter 3, we present a sensitive method to measure the INL of DTCs, based on phase modulation, and capable to achieve a time resolution of a few fs, by observing the frequency spectrum. This is $1-2$ orders of magnitude better than what is achievable with high-speed sampling oscilloscopes. After verifying the method with behavioral simulations, we used it to measure the INL of a constant-slope DTC for Phase-Locked Loop (PLL) applications, with a delay programmable from 19 ps to 189 ps with a resolution from 19 fs to $185 \mathrm{fs}$. Measurements with a $55 \mathrm{MHz}$ crystal clock demonstrate a INL of $1.77 \%$ LSB at 189 ps full-scale delay and $3.37 \%$ LSB at 19 ps, representing $8-9$ bit effective INL-limited resolution. [CP:2] .

As mentioned above, the targeted applications often require clocks in the low GHz range and we explore in this thesis the feasibility of a DFC exploiting constant-slope DTCs for those frequencies. Achieving such operating frequencies in a linear constant-slope DTC is challenging. In fact, a DTC cannot provide the entire working period fully programmable, as some overhead for initial voltage generation and post-delay reset is needed. Hence at least 2 DTCs need to be interleaved to ensure full-time availability. Architectural considerations regarding the DTC are discussed in chapter 4 (section 4.3.3). In the same chapter, we set up a behavioral model of the entire synthesizer, consisting of a Pulse-Output DFC generating square waves, with a deterministic DTC-based spur correction scheme. Chapter 4 also provides an analytical analysis of the output spectrum, that relates the DTC impairments to the output Spurious-Free Dynamic Range (SFDR) of the DFC. The results depend exclusively on the ratio between the output frequency and the clock frequency and on the DTC features (number of bits, INL and other impairments). An approximation for the worst-case spur can be determined for the case of an ideal DTC limited only by quantization noise, given the number of bits of the DTC and the frequency control word (section 4.4.2). The DTC INL can also be taken into account in the worstcase spur estimation (section 4.4.5), so that the DTC INL requirement can be derived, given a SFDR target. The maximum spur strength, in $\mathrm{dBc}$, increases with the frequency control word by $6 \mathrm{~dB}$ per octave. Simulations indicate that with a 12-bit counter, 11-bit DTC and a few LSBs timing errors a $60 \mathrm{~dB}$ clean spectrum is feasible (Fig. 4.13).

The actual design of DTC-based frequency synthesizer faces various challenges mainly related to the integration of the digital core with the DTC and DTC calibration (section 5.3.1). The design of the prototype 
to verify the concept of spur-correction with a DTC has been detailed in chapter 5, in $65 \mathrm{~nm}$ CMOS technology. It consists of a phase accumulator and a constant-slope high-speed DTC, with a coarse-fine scheme to produce delay (section 5.1). Measurements show it operates correctly at $1.2 \mathrm{~V}$, with frequency range $342 \mathrm{kHz}-700 \mathrm{MHz}$. The effectiveness of the DTC correction mechanism on the output spurs relies on the DTC resolution and linearity. The design of the DTC in the prototype is sub-optimal in terms of power consumption, thus affecting the system Figure-of-Merit (Table 5.4). The DFC prototype shows unexpected bumps in the profile of the maximum output spur that can be due to errors in the DTC delay word and that could be solved with the inclusion of the coarse DTC in the automated digital designed block, for a more robust static timing analysis. Due to these bumps, the prototype achieves $-29.7 \mathrm{dBc}$ SFDR at an output frequency of $449 \mathrm{MHz}$ and $\mathrm{SFDR}=-44.8 \mathrm{dBc}$ at $\mathrm{f}_{\mathrm{DDS}}=499.7 \mathrm{MHz}$, with a power consumption of $51 \mathrm{~mW}$ excluding the measurement output buffers. The speed bottleneck in the current DFC implementation is given by the DTC, when the system input reference frequency exceeds an upper limit at around 2.8 GHz (section 5.7.7). Overall, it can be concluded that the prototype needs further improvements to be competitive with the state-of-the-art, but the approach of DTC correction for spur reduction is still promising to realize flexible and compact digitally programmable frequency synthesis. Attractive aspects of a DFC with DTC-based spur correction is the avoidance of reconstruction filters compared to traditional sinewave DFCs and possibility of coexistence of multiple DDS blocks to generate all the frequencies required in a System-on-Chip (SoC), thus avoiding coupling issues of multi-PLL analog approaches. Orthogonal techniques like dithering or $\Sigma \Delta$ modulation of the input word FCW can be added to the DTC correction, in order to further improve the output spectrum at the expense of the output noise floor. However, the introduction of these techniques would require the analysis in chapter 4 to be adapted to include such extra effects.

\subsection{Original contributions}

The work presented in this thesis contains several original contributions:

- The introduction of a measurement method in the frequency domain to measure the DNL and INL of DTCs with fs resolution. The method is based on phase modulation theory, exploiting the properties of averaging and narrow-band measurements compared to a sampling device/oscilloscope-based approach. We provide the mathematical foundation of our method, behavioral proof and experimental application. (chapter 3 and [CP:1]).

- The introduction of a DTC as an alternative to a Delay-Locked Loop (DLL) for correction of the output spurs in a Pulse-Output DFC; 
(chapter 4 and $[C P: 3])$

- The development of a mathematical analysis of the output spurs in a DTC-based Pulse-Output DFC, with behavioral proof; (chapter 4 and [CP:3] )

- The introduction of time-interleaving and coarse/fine delay mechanism to tackle the fundamental problem of dead-time in constantslope DTCs [CP:2], thus allowing to incorporate such linear DTCs inside a DFC synthesizer; (chapter 4 and [CP:3] )

- The introduction of closed-form design equations, that allow to predict the maximum output spur of a DTC-based Pulse-Output DFC, knowing the DTC quantization and INL; (chapter 4 and [CP:3])

- The introduction of a high-speed complementary-slope DTC with starting voltage levels set by custom capacitive Digital-to-Analog Converters (DACs). (chapter 5)

\subsection{Recommendations}

This thesis has shown the potential of DTCs for deterministic correction of the spurs produced by Pulse-Output DFCs. The ultimate goal is to implement a set of digital synthesizers, all derived from a single integer-N PLL, with spectral purity at least comparable or even better than squarewave generators based on fractional-N PLLs only. This is challenging since digital synthesizers are heavily affected by deterministic jitter, essentially due to the quantized nature of their digital core. A further developing on the topic of this thesis is the way to go to make digital synthesizers commercially competitive.

From a theoretical point of view, the analysis in chapter 4 [CP:3] focused on the spur strength and on a closed-form expression to quickly calculate the worst spur. We recommend a further development of the analysis to determine also the position of the worst spur with a simple closed-form expression. This would be helpful to easily identify cleaner bands depending on the input word FCW and can pave the way to new spur-canceling techniques.

As for the circuit implementation of the DFC, we recommend a design refinement based on an iterative design and layout, in order to minimize the parasitics and to improve the power efficiency. In fact, in the current implementation the dominant power contributor of the analog supply $\mathrm{VDD}_{\mathrm{A}}$ is the DTC current source (Fig. 5.45). Other weaknesses of the DFC-prototype are the lack of calibration capacitors to compensate the mismatches between the R and F sides of the DTC (see Fig. 5.1 for reference) and differences in the delay paths of MR and MRN that should be minimized. Besides these improvements that can be easily done, the sensitivity of the circuit to PVT variations can be addressed by doing a 
background calibration with slow, low-power closed loops. Notice that the DTC will still be open loop and therefore intrinsically different from a DLL, as discussed in section 4.2 , but the calibration will be constantly adjusted. A more efficient implementation of the coarse DTC in the digital domain would avoid the observed time-violations in the fine-DW sampling, thus substantially improving the spur reduction (Fig. 5.46).

Another recommendation is the study of the behaviour that is still not understood in the prototype, namely the jumps in the measured spurs for the same code observed in Fig. 5.29 and Fig. 5.30, as well as the bumps in the maximum measured spurs in Fig. 5.38.

Being able to push down the spurs at around the level of noise floor a new interesting aspect can be investigated. Indeed, while in the current DFC-prototype the jitter contribution is secondary compared to the spurs, random noise becomes relevant when deterministic spurs are low enough. In this case, we recommend to extend the deterministic analysis in chapter 4 with a statistical one. Our expectation is that thermal noise would act as dithering for the spurs, improving the output SFDR. 



\section{APPENDIX}

\section{Implementation details}

In this appendix, we will discuss some implementation details of the prototype UTDUCK that are relevant for some interested in using the idea proposed in this thesis.

\section{A.1 Capacitive DAC}

In section 5.3.6 we discussed the capacitive DACs in the prototype, focusing on their implementation and driving scheme. Here we will consider layout details and other aspects.

As shown in section 5.3.6, each of the two DACs consists of the two sets of weighted capacitors (Fig. 5.11). One set of capacitor is represented in Fig. A.1a to show the relative size. The unary MSBs capacitors are highlighted in red. The extra capacitor, in gray, has the same weight (i.e. area) of the sum of all the other capacitors.

The scheme in Fig. A.1a is not the true layout used for the prototype. The actual layout of one capacitor matrix is shown in Fig. A.1b in a common centroid arrangement. The numbers inside each cell in the matrix refers to the bit connected to it. For example, the white cells 0 are connected to the data bit $b_{0}$. A unit capacitance $C_{u}$ in Fig. 5.11 corresponds to two cells in the matrix. This choice allows a better scrambling of the cells and common centroid layout. The light-blue cells $b_{\mathfrak{u}}$ at the two extreme sides correspond to the unit capacitance that is switched for odd values of DW. One matrix corresponds to one set of weighted capacitors, so each DAC is made up of two matrices.

The schematic and 3-D layout of the unit cell in the matrix is shown in Fig. A.2. Each cell consists of an inverter and a custom plate capacitance built on top of it, in a modular arrangement. Three input data lines are placed between the inverter and the bottom plate. Each cell is connected to one of the three data line, according to the pattern in Fig. A.1b. To increase 


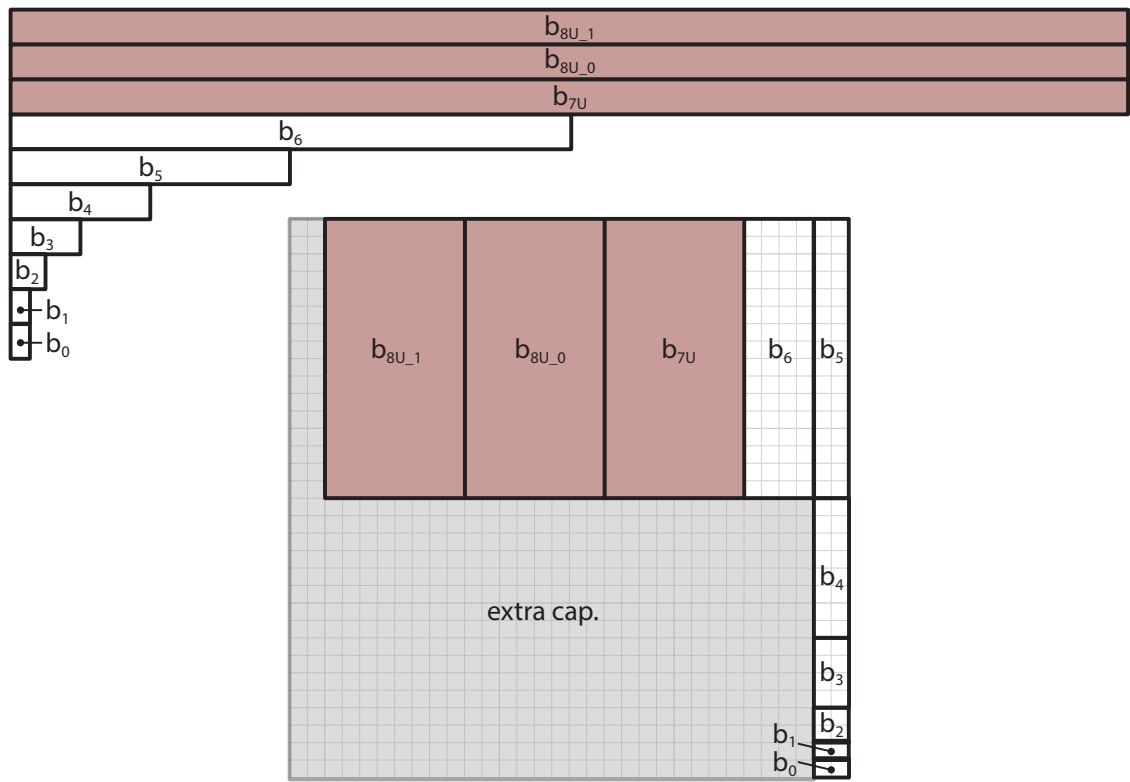

(a) Capacitor weights.

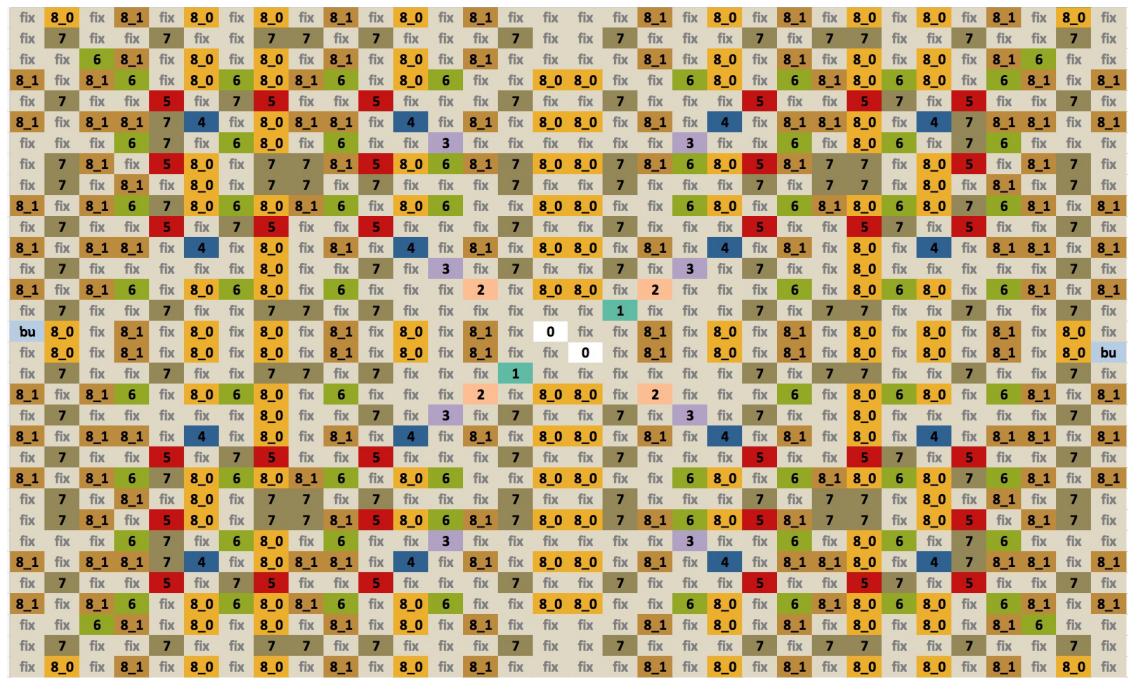

(b) Matrix common-centroid layout.

Figure A.1: Scaled area of capacitors and matrix layout of the 9-bit capacitive DAC.

the capacitance density, the top plate (gray in Fig. A.2b) is in the middle of two layers of bottom plate and connected to the output line through a via in the center. The value of the unit capacitance is twice the value of the cell capacitance, so $\mathrm{C}_{\mathrm{U}}=3.56 \mathrm{fF}$.

The capacitive DAC has been designed to draw a code-independent current from the reference voltage. Depending on the delay word DW, a 


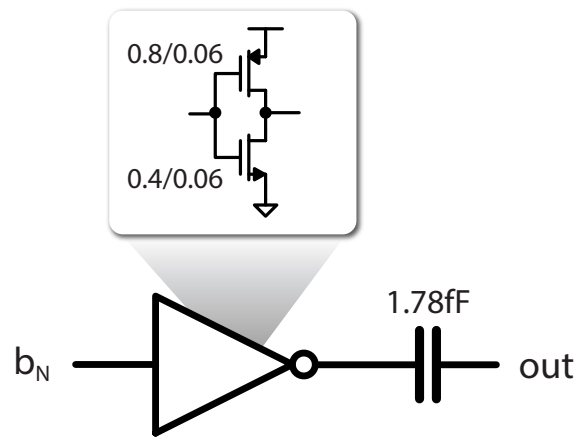

(a) Schematic
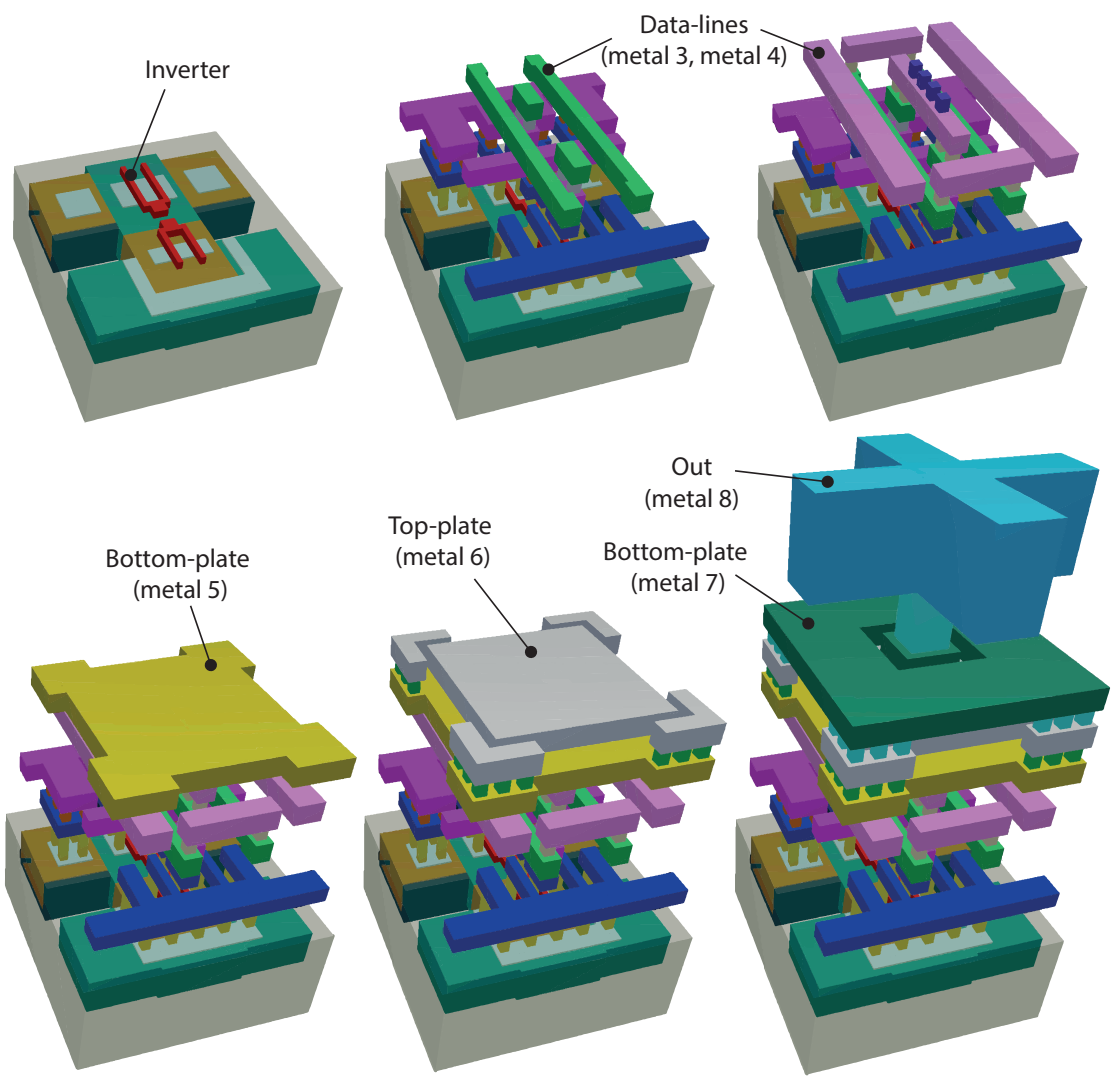

(b) 3-D layout.

Figure A.2: Unit cell of the capacitive DAC. The different colors refer to different metal layers.

ripple in the reference voltage can still appear as differential mode at the input of the Zero Crossing Detector (ZCD), because the capacitances at nodes $A$ and $B$ are connected to two complementary words that are different by definition. This can be easily observed in the simplified scheme in Fig. 5.10 that considers a 3-bit DAC for clarity: the number of 1s connected 


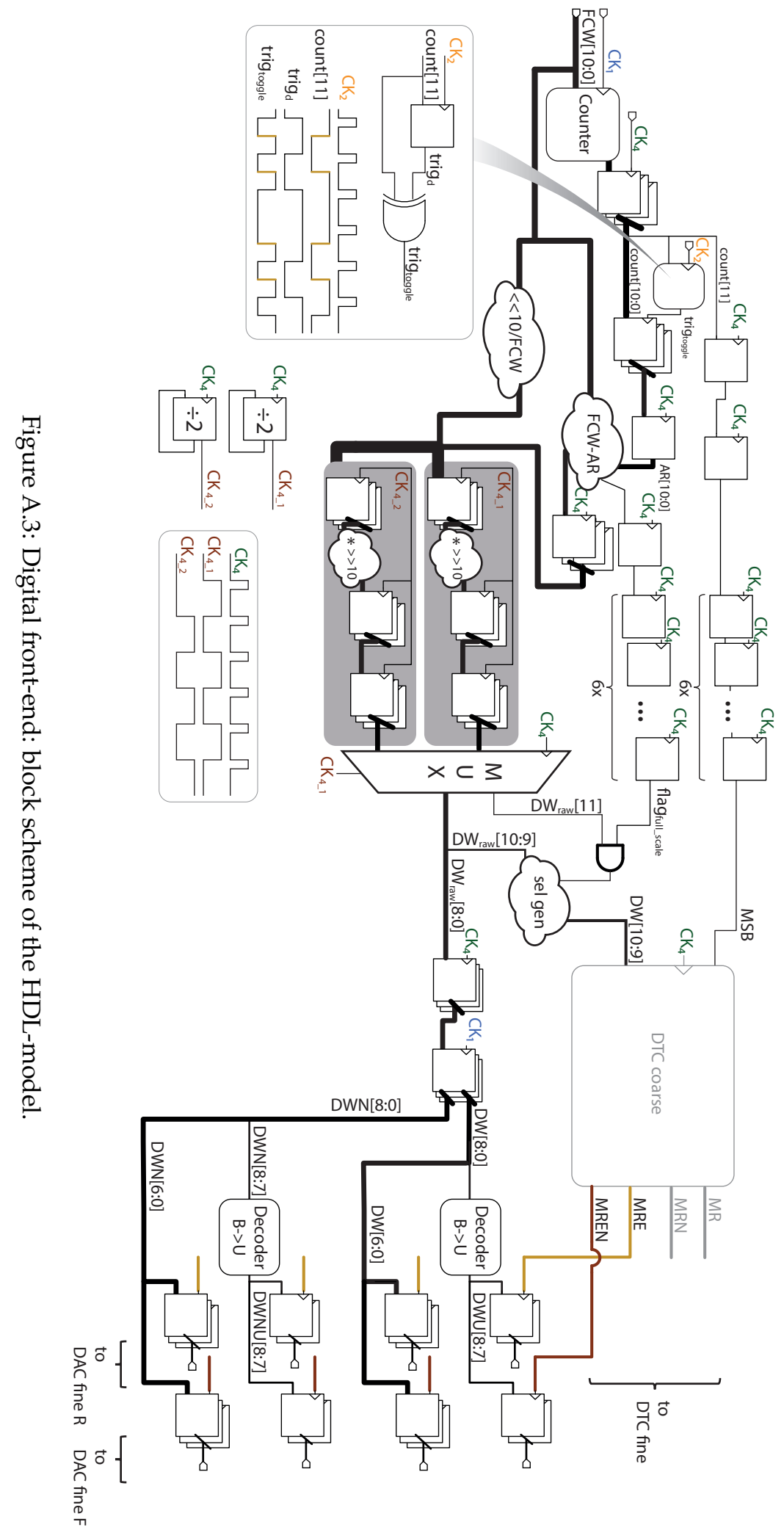


to nodes $A$ and $B$ are generally different for each code, meaning that any eventual disturbance on the reference voltage will couple with different weights on the two nodes $A$ and $B$, depending on the code DW. The case DW $=0$ in Fig. 5.10 leads to the canceling of the reference ripple, while the maximum DW will not benefit from the differential reading of the DAC output. Simulations with estimated parasitic inductors for the bond wires, shown in section 5.6 (Fig. 5.20) do not show voltage ripples in the reference voltage triggered by that the DAC conversion.

\section{A.2 Digital front-end}

The digital part of the DFC system shown in section 5.1 (Fig. 5.1) has been detailed in Fig. A.3. The digital block includes the Pulse-Output Digitalto-Frequency Converter (DFC) (counter) and resamplers to be connected to the DACs of the fine-Digital-to-Time Converters (DTCs). It has been synthesized with digital tools, using the standard Low-Voltage (LVT) library of TSMC65 technology, starting from a HDL-model, illustrated as a block scheme in Fig. A.3, whose Verilog code is detailed in appendix D. The model reflects the Pulse-Output DFC discussed in chapter 4. It contains a 12-bit counter whose MSB bit, count [11] (that can be considered the overflow of a 11-bit counter), is resampled multiple times to meet the timing requirements and then sent to the coarse-DTC. The remaining bits from the counter, count $[10: 0]$, are evaluated by sampling them with the signal trig toggle $_{\text {, }}$ that has a rising edge at every edge of count [11]. The resulting residue AR $[10: 0]$ is processed in combinational blocks (shown as clouds in Fig. A.3) to determine the current Delay Word DW to be sent to the DACs of the fine-DTCs. Pipelining and time-interleaving (gray blocks in Fig. A.3) have been necessary to meet the timing constraints. A multiplexer recombines the two interleaved paths to produce the word $D W_{\text {raw }}[11: 0]$. The word is then resampled multiple times and converted to the right format, with two unary bits, to drive the DACs. In the last set of flip-flops, the digital word is resampled with the signals MRE and MREN coming from the coarse-DTC.

\section{A.3 Calibration}

The calibration procedure has been motivated in section 5.7.2. Most of the calibration can be done digitally, by programming specific bytes placed at different points of the prototype. This is shown in Fig. A.4 (which is an expanded version of Fig. 5.1), where the blocks containing the calibration bytes have been detailed and the bytes are highlighted in blue. The focus of Fig. A.4 is to show the position of the calibration bytes inside the overall architecture, while each individual block can be seen with full details in Fig. 5.2, Fig. 5.4, and Fig. 5.14, respectively. 


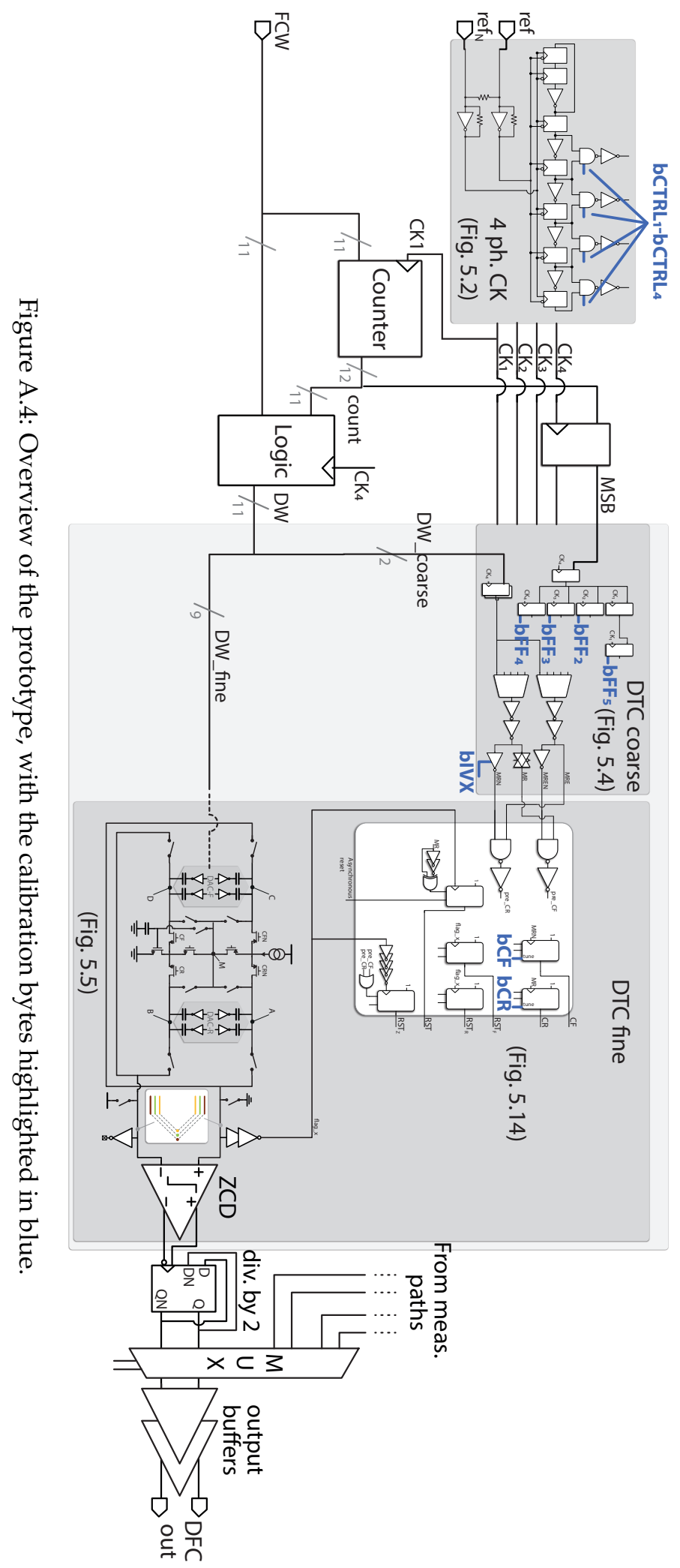




\begin{tabular}{|c|l|}
\hline Calibration byte & \multicolumn{1}{|c|}{ Function } \\
\hline bCTRL $_{x} \quad x=\{1,2,3,4\}$ & Align the clock phases to each other \\
\hline bFF $_{x} \quad x=\{2,3,4,5\}$ & Adjust rising edges of MREN and MRN \\
\hline bCR, bCF & Adjust starting of the ramps \\
\hline bIVX & Adjust rising edges of MRN \\
\hline
\end{tabular}

(a) List of the calibration bytes with their function.

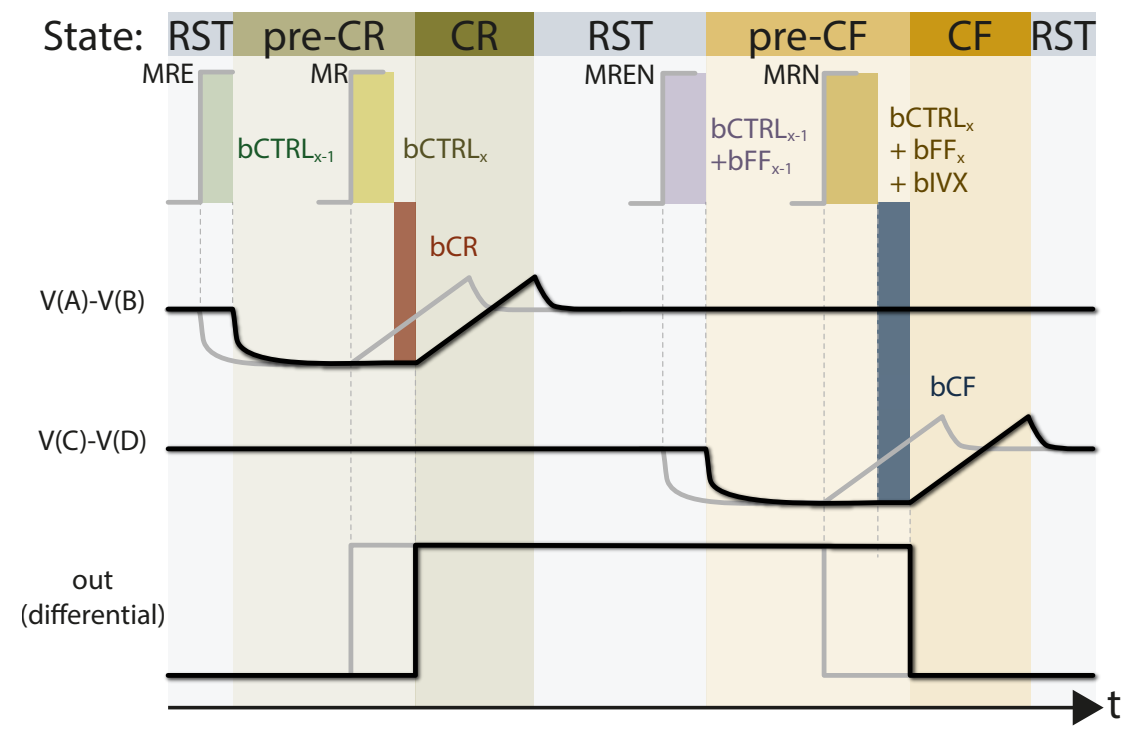

(b) Effect on the main waveforms in the circuit.

Figure A.5: Calibration bytes and their effect on the main waveforms in the circuit. A full cycle of states has been considered

Fig. A.5 lists the calibration bytes and their function, as well as their effect on the main waveforms in the circuit. The gray signals in Fig. A.5 refer to the uncalibrated case, while the black waveforms show the effect of each calibration byte. For simplicity, the gate delays have been ignored and only the delays due to the calibration bytes are considered in Fig. A.5. Thus, for example, the gray ramps are perfectly aligned with the rising edges of $M R$ and MRN. The calibration bytes $b C T R L_{x}$, with $x \in\{1,2,3,4\}$, regulate the alignment of the four clock phases ${ }^{\dagger}$. Therefore, they control the edges of the signals responsible for the DAC conversion (MRE, MREN), and for the ramp starting (MR, MRN), as shown in Fig. A.5. The other calibration bytes can be used to regulate the output duty-cycle: the bytes

\footnotetext{
$\dagger$ Actually these bytes regulate the pull-down of the NAND gates in Fig. 5.2.
} 


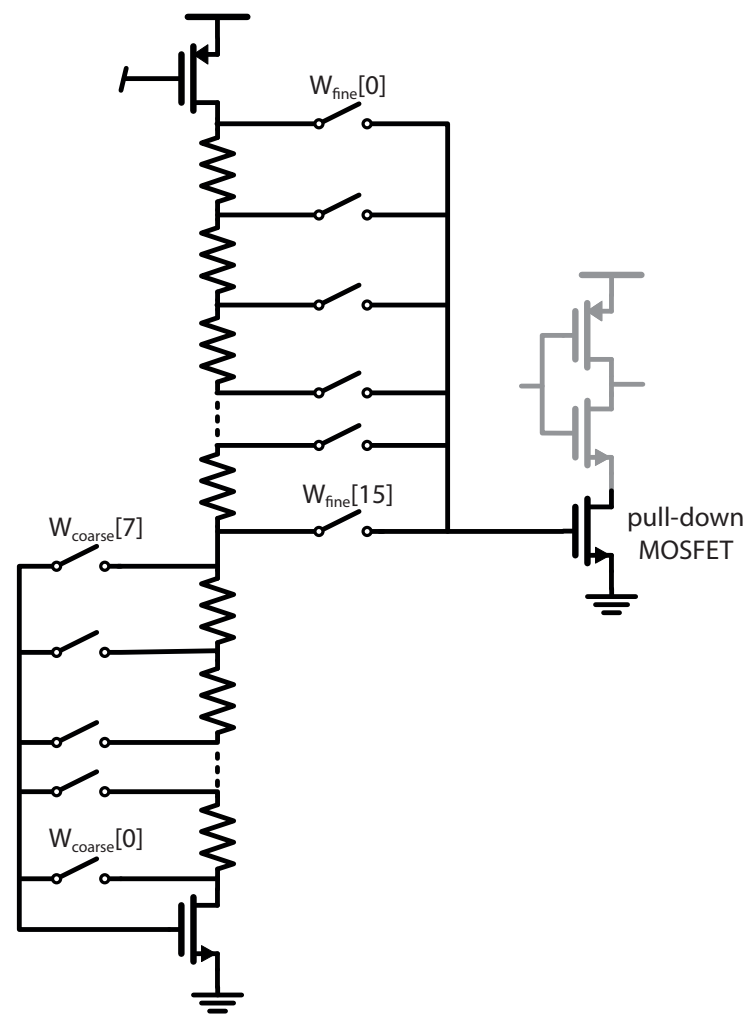

Figure A.6: Pull-down current starving controlled by a resistor ladder, for calibration.

$\mathrm{bFF}_{x}$, with $x \in\{2,3,4,5\}$ (see Fig. A.4), act only on the pull-down during the resampling of the signal MSB, thus they only act on the rising edges of the signals MREN and MRN (see Fig. A.5); the byte bIVX only controls MRN (Fig. A.4 and Fig. A.5); the bytes bCF and bCR adjust the starting time of the ramps and, therefore, the start of the states $C F$ and $C R$, respectively (Fig. A.4 and Fig. A.5). Finally, the fine-scale delay is controlled analogically, by adjusting the core current.

The basic mechanism of all the aforementioned calibration bytes is the adjustment of the pull-down delay through current starving, as shown in Fig. A.6. The pull-down MOSFET has its gate connected to a resistor ladder. The active taps of the ladder depend on the calibration word, that is divided in coarse and fine bits. Therefore, for each calibration word, there is always one coarse and one fine-tap active (i.e. closed switch). The active coarse-tap determines the $V_{\mathrm{GS}}$ of the nMOS at the bottom of the ladder, which is matched with the pull-down MOSFET. Fig. A.6 shows 3 coarse-bits and 4 fine-bits, but in the prototype there are also bigger ladders with 4 coarse-bits and 4 fine-bits.

The calibration procedure consists on the following sequence of steps, that are performed on the prototype in measurement-mode: 
Table A.1: CALIBRATION VALUES FOR $\mathrm{f}_{\mathrm{ref}}=2 \mathrm{GHz}$.

\begin{tabular}{|c|c|c|c|}
\hline Parameter & Value & Parameter & Value \\
\hline bCTRL $_{1}$ & 138 & bFF $_{2}$ & 0 \\
\hline bCTRL $_{2}$ & 154 & bFF $_{3}$ & 0 \\
\hline bCTRL $_{3}$ & 150 & bFF $_{4}$ & 0 \\
\hline bCTRL $_{4}$ & 53 & bFF $_{5}$ & 0 \\
\hline bCR & 127 & blVX & 0 \\
\hline bCF & 0 & Iref $_{\text {core }}$ & $475 \mathrm{uA}$ \\
\hline
\end{tabular}

step 1 Duty-cycle calibration. With FCW set to a power of 2, for example $F C W=1024$, fix the DTC input word to DW $=0$ (phase $C_{2}$ selected) and choose $\mathrm{bFF}_{2}, \mathrm{bIVX}, \mathrm{bCF}, \mathrm{bCR}$ to minimize the even harmonics. The input FCW will not be changed in the following steps.

step 2 Duty-cycle calibration. Repeat step1 with DW $=512$ (phase $\mathrm{CK}_{3}$ selected), DW $=1024$ (phase $\mathrm{CK}_{4}$ selected), DW $=1536$ (phase $\mathrm{CK}_{1}$ selected), and regulate $\mathrm{bFF}_{3}, \mathrm{bFF}_{4}$ and $\mathrm{bFF}_{5}$, respectively, to minimize the even harmonics.

step 3 Fine-scale calibration. Use the modulation method in [CP:1] with DW switching between two values and act on the core current $\mathrm{I}_{\text {ref }}$ core (Fig. 5.8) to measure a spur corresponding to the expected delay. With DW switching between $0-511$ (fine-full scale), the expected delay is $\mathrm{T}_{\text {ref }} / 2-1 \mathrm{LSB}$, but the measurement would have low sensitivity. To decrease the measured uncertainty, a smaller code-step can be used, $i$. e. $\mathrm{DW}=0-8$, corresponding to an expected delay of $\mathrm{T}_{\text {ref }} / 2 / 2048 * 8$.

step 4 Coarse step calibration. Use the modulation method in [CP:1], switching between two adjacent clock phases, and act on bCTRL $L_{x}$, with $x \in\{1,2,3,4\}$, to correctly align the clock phases. As in step 3, a code-step 8 can be used for high sensitivity. Thus:

- $\mathrm{DW}=504\left(\mathrm{CK}_{2}\right)-512\left(\mathrm{CK}_{3}\right)$ acting on $\mathrm{bCTRL_{2 }}$ and $\mathrm{bCTRL} \mathrm{L}_{3}$ to measure the expected delay of $\mathrm{T}_{\text {ref }} / 2 / 2048 * 8$;

- $\mathrm{DW}=1016\left(\mathrm{CK}_{3}\right)-1024\left(\mathrm{CK}_{4}\right)$ acting on $\mathrm{bCTRL} 4$;

- $\mathrm{DW}=1528\left(\mathrm{CK}_{2}\right)-1536\left(\mathrm{CK}_{3}\right)$ acting on $\mathrm{bCTRL} \mathrm{L}_{1}$.

The calibration procedure has been executed with $f_{\text {ref }}=2 \mathrm{GHz}$. The resulting values are listed in Table A.1. An exact $50 \%$ output duty-cycle could not be achieved, reasonably as a result of a systematic delay between $M R$ and MRN, due to layout asymmetries in the two paths. The calibration 
bytes controlling the duty cycle has been used to partially compensate this systematic delay, by setting $\mathrm{bFF}_{x}, \mathrm{bIVX}, \mathrm{bCF}$ to the minimum value 0 and $\mathrm{bCR}$ to the maximum value 127 . In this way, a second harmonic of $\sim-50 \mathrm{dBc}$ has been achieved, corresponding to a duty-cycle error of $0.1 \%$ according to Fig. 4.14. Moreover, the calibration bytes in the current implementation of the prototype do not correct capacitance mismatches between the two sides ( $R$ and F) of the fine-DTC, so the consequent extra fractional spurs, shown in Fig. 4.13, cannot be modified.

When changing $\mathrm{f}_{\text {ref, }}$, the fine-DTC full-scale should be re-calibrated by adjusting the core reference current $\mathrm{I}_{\text {ref }_{\text {core }}}$ (shown in Fig. 5.8). Therefore, the core current should be increased from the value $475 \mu \mathrm{A}$ in Table A.1, that is valid for $f_{\text {ref }}=2 \mathrm{GHz}$. For measurements at the maximum reference frequency $f_{\text {ref }_{\max }}=2.8 \mathrm{GHz}$ (Fig. 5.41), it is $\mathrm{I}_{\text {ref }_{\text {core }}}=780 \mu \mathrm{A}$, while the other calibration parameters have been kept the same as in Table A.1. 


\section{Mathematical derivations for the analysis in chapter 4}

\section{B.1 Derivation of (4.13) and (4.14)}

Following similar steps as for the Digital-to-Period Converter (DPC) in [44] and referring to Fig. 4.8, , the ideal edge timing is:

$$
t_{\text {ideal }}[l]=\frac{l-1}{2} T_{D D S}
$$

With $l \geqslant 1$, rising edges for $l$ odd, falling edges for $l$ even. We can define the time-quantization error between corresponding edges of the real and ideal waveforms as:

$$
\tau_{\mathrm{q}}[\mathrm{l}] \triangleq \mathrm{t}[\mathrm{l}]-\mathrm{t}_{\text {ideal }}[\mathrm{l}]
$$

The signal $s$ in Fig. 4.8 can be written as:

$$
s(\eta)=A\left\{\sum_{\substack{l=1 \\ l \text { odd }}}^{\mathrm{L}} u(\eta-t[l])-\sum_{\substack{l=1 \\ l \text { even }}}^{\mathrm{L}} u(\eta-t[l])\right\}
$$

Where $\eta$ is the time variable, $u(\eta)$ is the step function, $A$ is the signal amplitude and L is defined in (4.10). Using (B.1) and (B.2), the Fourier transform of $s$ is

$$
\begin{aligned}
S(f)=\frac{A}{j 2 \pi f} & \left\{\sum_{\substack{l=1 \\
l \text { odd }}}^{L} e^{-j 2 \pi f\left(\tau_{\mathrm{q}}[l]+\frac{l-1}{2} T_{D D S}\right)}\right. \\
& \left.-\sum_{\substack{l=1 \\
l \text { even }}}^{L} e^{-j 2 \pi f\left(\tau_{\mathrm{q}}[l]+\frac{l-1}{2} \mathrm{~T}_{\mathrm{DDS}}\right)}\right\}
\end{aligned}
$$

This appendix has been previously published in [CP:3] . We added here a few more steps in the mathematical derivation that have been omitted in [CP:3] due to space limitation. 
The output is described by (4.11) and (4.12). The weights $X_{h}$ can be calculated as [103]:

$$
X_{h}=\frac{1}{G_{R R} T_{C K}} S\left(\frac{h}{\operatorname{GRR}_{C K}}\right)
$$

Combining (B.4) and (B.5) it is

$$
\begin{aligned}
X_{h}=\frac{A}{j 2 \pi h} & \left\{\sum_{\substack{l=1 \\
l \text { odd }}}^{L} e^{-j 2 \pi \frac{h}{L}(l-1)} e^{-j 2 \pi \frac{h}{G R R T_{C K}} \tau_{q}[l]}\right. \\
& \left.-\sum_{\substack{l=1 \\
l \text { even }}}^{L} e^{-j 2 \pi \frac{h}{L}(l-1)} e^{-j 2 \pi \frac{h}{\text { RRR TK }_{C}} \tau_{q}[l]}\right\}
\end{aligned}
$$

Where the equality GRR $\mathrm{T}_{\mathrm{CK}}=\frac{\mathrm{L}}{2} \mathrm{~T}_{\mathrm{DDS}}$ has been used. By considering only the sub-harmonics $(h<L / 2)$ and being $\tau_{\mathrm{q}} / \mathrm{T}_{\mathrm{CK}}<<1$ for all $l$, we can approximate the complex exponential:

$$
e^{-j 2 \pi \frac{h}{\operatorname{GRRT}_{C K}} \tau_{\mathrm{q}}[l]} \approx 1-j 2 \pi \frac{\mathrm{h}}{\mathrm{GRR}_{\mathrm{CK}}} \tau_{\mathrm{q}}[\mathrm{l}]
$$

Therefore, (B.6) becomes

$$
\begin{aligned}
X_{\mathrm{h}} \approx-\frac{A}{\mathrm{GRR} T_{\mathrm{CK}}} & \left\{\sum_{\substack{l=1 \\
l \text { odd }}}^{\mathrm{L}} \tau_{\mathrm{q}}[l] e^{-j 2 \pi \frac{\mathrm{h}}{\mathrm{L}}(l-1)}\right. \\
& \left.-\sum_{\substack{l=1 \\
l \text { even }}}^{L} \tau_{\mathrm{q}}[l] e^{-j 2 \pi \frac{\mathrm{h}}{\mathrm{L}}(l-1)}\right\}
\end{aligned}
$$

We can define the quantization error $q_{e}[l]$

$$
q_{e}[l] \triangleq(-1)^{l+1} \frac{\tau_{q}[l]}{\frac{T_{C K}}{2^{N \text { DTC }}}}
$$

Thus, (B.8) can be written as

$$
X_{h} \approx-\frac{A}{G R R T_{C K}} \frac{T_{C K}}{2^{N_{\text {DTC }}}}\left\{\sum_{l=1}^{L} q_{e}[l] e^{-j 2 \pi \frac{h}{L}(l-1)}\right\}
$$

that is equivalent to (4.13) and (4.14).

\section{B.2 Proof of the approximation (4.18)}

We will prove (4.18):

$$
\max _{h}\left|\sum_{l=1}^{L / 2} e^{-j 2 \pi \frac{h}{L} l}\right| \approx \frac{L}{\pi}
$$


We can rewrite the sum in (B.11) as

$$
\sum_{l=0}^{L / 2-1} e^{-j 2 \pi \frac{h}{L}(l+1)}=e^{-j 2 \pi \frac{h}{L}} \sum_{l=0}^{L / 2-1} e^{-j 2 \pi \frac{h}{L} l}
$$

From [117]

$$
\sum_{l=0}^{\mathrm{L} / 2-1} e^{-j 2 \pi \frac{\mathrm{h}}{\mathrm{L}} \mathrm{l}}=\frac{\sin \left(\frac{\pi \mathrm{h}}{2}\right)}{\sin \left(\frac{\pi \mathrm{h}}{\mathrm{L}}\right)} e^{-\mathrm{j} 2 \pi \frac{\mathrm{h}}{\mathrm{L}} \frac{\mathrm{L} / 2-1}{2}}
$$

Therefore

$$
\begin{aligned}
\max _{h}\left|\sum_{l=1}^{L / 2} e^{-j 2 \pi \frac{h}{L} l}\right| \\
=\underbrace{\left|e^{-j 2 \pi \frac{h}{L}}\right|}_{1} \underbrace{\left|e^{-j 2 \pi \frac{h}{L} \frac{L / 2-1}{2}}\right|}_{1} \max _{h}\left|\frac{\sin \left(\frac{\pi h}{2}\right)}{\sin \left(\frac{\pi h}{L}\right)}\right|
\end{aligned}
$$

In the right-hand term of (B.14) the maximum value is obtained for the smallest odd value of $h$, i.e. $h=1$ :

$$
\max _{h}\left|\frac{\sin \left(\frac{\pi h}{2}\right)}{\sin \left(\frac{\pi h}{L}\right)}\right| \stackrel{h=1}{=} \frac{1}{\left|\sin \left(\frac{\pi}{L}\right)\right|} \approx \frac{1}{\frac{\pi}{L}}=\frac{L}{\pi}
$$

Where the approximation of the sine with its argument is good for large L, i.e. all practical values of FCW.

We can also prove:

$$
\max _{h}\left|\sum_{l=1}^{L / 2}(-1)^{l} e^{-j 2 \pi \frac{h}{L} l}\right| \approx \frac{L}{\pi}
$$

The sum in (B.16) can be written as:

$$
\begin{aligned}
\sum_{l=0}^{\mathrm{L} / 2-1}(-1)^{l+1} & e^{-j 2 \pi \frac{\mathrm{h}}{\mathrm{L}}(l+1)} \\
& =(-1) e^{-j 2 \pi \frac{\mathrm{h}}{\mathrm{L}}} \sum_{l=0}^{\mathrm{L} / 2-1}(-1)^{l} e^{-j 2 \pi \frac{\mathrm{h}}{\mathrm{L}} l}
\end{aligned}
$$

From [117]

$$
\begin{array}{r}
\sum_{l=0}^{L / 2-1}(-1)^{l} e^{-j 2 \pi \frac{h}{L} l}=\frac{1-(-1)^{\frac{L}{2}} e^{-j \pi h}}{1-(-1) e^{-j 2 \pi \frac{h}{L}}} \\
=\frac{1+e^{-j \pi h}}{1+e^{-j 2 \pi \frac{h}{L}}}
\end{array}
$$


Where the last equality comes from L/2 being odd. Therefore:

$$
\begin{aligned}
\max _{h}\left|\sum_{l=1}^{L / 2}(-1)^{l} e^{-j 2 \pi \frac{h}{L} l}\right| \\
=|-1| \underbrace{\left|e^{-j 2 \pi \frac{h}{L}}\right|}_{1} \max _{h}\left|\frac{1+e^{-j \pi h}}{1+e^{-j 2 \pi \frac{h}{L}}}\right|
\end{aligned}
$$

The right-hand side of (B.19) is zero for $h$ odd. The maximum value is obtained for the even value of $h$ that is closest to $L / 2$, i.e. $h=L / 2 \mp 1$. Thus it is:

$$
\begin{gathered}
\max _{h}\left|\frac{1+e^{-j \pi h}}{1+e^{-j 2 \pi \frac{h}{L}}}\right| \stackrel{h=\frac{L}{=} \mp 1}{=} \frac{2}{\left|1-e^{ \pm j \frac{2 \pi}{L}}\right|}=\frac{2}{\sqrt{2-2 \cos \left( \pm \frac{2 \pi}{L}\right)}} \\
\approx \frac{2}{\sqrt{2-2\left(1-\frac{1}{2}\left(\frac{2 \pi}{L}\right)^{2}\right)}}=\frac{2}{\frac{2 \pi}{L}}=\frac{L}{\pi}
\end{gathered}
$$

Where the Taylor expansion of the $\operatorname{cosine} \cos (x) \approx 1-\frac{x^{2}}{2}$ is good for all practical values of FCW. The proof of:

$$
\max _{h}\left|\sum_{l=1}^{L / 2}(-1)^{l+1} e^{-j 2 \pi \frac{h}{L} l}\right| \approx \frac{L}{\pi}
$$

follows immediately, since

$$
\begin{aligned}
\left|\sum_{l=1}^{\mathrm{L} / 2}(-1)^{\mathrm{l}+1} e^{-j 2 \pi \frac{\mathrm{h}}{\mathrm{L}} \mathrm{l}}\right| & =\left|-1 \sum_{\mathrm{l}=1}^{\mathrm{L} / 2}(-1)^{\mathrm{l}} e^{-\mathrm{j} 2 \pi \frac{\mathrm{h}}{\mathrm{L}} \mathrm{l}}\right| \\
& =\left|\sum_{l=1}^{\mathrm{L} / 2}(-1)^{\mathrm{l}} e^{-j 2 \pi \frac{\mathrm{h}}{\mathrm{L}} \mathrm{l}}\right|
\end{aligned}
$$

which leads back to (B.16).

\section{B.3 Expected value of Integral Non-Linearity (INL)}

From the theory of probability [113], given a random variable $\tilde{x}$ and a function $g($.$) , the expected value of the random variable \tilde{y}=g(\tilde{x})$ can be calculated from the following equation ((5-55) in [113]):

$$
E\{g(x)\}=\int_{-\infty}^{+\infty} g(x) \xi_{x}(x) d x
$$

where $\xi_{x}(x)$ is the probability density function (p.d.f.) of $\tilde{x}$. For a discretetype random variable $\tilde{x}, \xi_{x}(x)$ is called probability mass function (p.m.f.) 


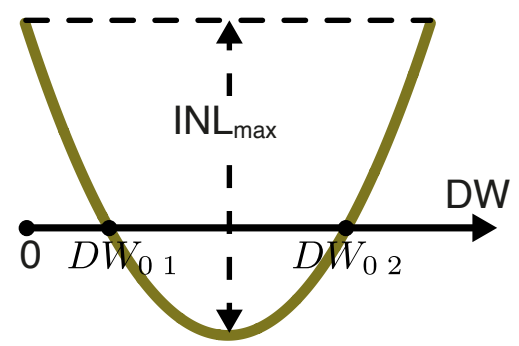

(a) INL (DW)

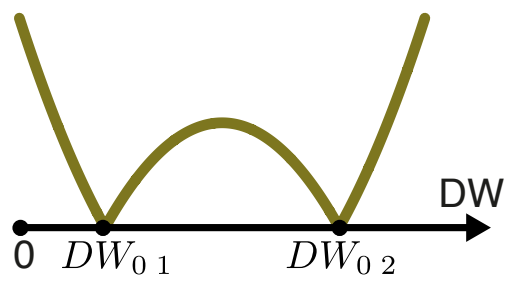

(b) $|\operatorname{INL}(\mathrm{DW})|$

Figure B.1: Parabolic INL and its absolute value.

and it is a sum of Dirac delta functions:

$$
\xi_{x}(x)=\sum_{i} P\left\{\tilde{x}=x_{i}\right\} \delta\left(x-x_{i}\right)
$$

Where $P\left\{\tilde{x}=x_{i}\right\}$ is the probability of the event $\left\{\tilde{x}=x_{i}\right\}$. Thus, for a discretetype random variable, (B.23) becomes:

$$
E\{g(x)\}=\sum_{i} g\left(x_{i}\right) P\left\{\tilde{x}=x_{i}\right\}
$$

Similarly, for a generic input frequency word FCW in a Digital-toFrequency Converter (DFC) with a block scheme like Fig. 4.7, we can consider DW a discrete uniform random variable DW (assuming only integer values), with p.m.f.:

$$
\xi_{\mathrm{DW}}(\mathrm{DW})=\sum_{i=1}^{2^{\mathrm{N}_{\mathrm{DTC}}-2}} \frac{1}{2^{\mathrm{N}_{\mathrm{DTC}}-2}} \delta(\mathrm{DW}-\mathrm{i})
$$

Where $\mathrm{N}_{\text {DTC }}-2$ is the number of bits of each fine Digital-to-Time Converter (DTC), that is the source of INL. Then $E\{|\operatorname{INL}(\mathrm{DW})|\}$ should be calculated from (B.25).

However, to simplify calculations, for $N_{\text {DTC }}$ of a few units, DW can be considered a continuous-type random variable, with uniform p.d.f.:

$$
\xi_{\mathrm{DW}}(\mathrm{DW})=\left\{\begin{array}{cc}
\frac{1}{2^{\mathrm{N} \mathrm{DTC}^{-2}}} & 0 \leqslant \mathrm{DW} \leqslant 2^{\mathrm{N}_{\mathrm{DTC}}-2} \\
0 & \text { otherwise }
\end{array}\right.
$$

And $E\{|I N L(D W)|\}$ can be calculated from (B.23). With $\mathrm{N}_{\mathrm{DTC}}>6$ the error in the calculation with (B.23) instead of (B.25) is $<1 \% \mathrm{INL}_{\max }$.

For the sub-harmonic power calculation in section 4.4.5 the INL function should have mean value 0 , since we are interested in the real error compared to the average delay and the calculations should be independent of delay offsets. A $0-$ mean parabolic INL, with axis in DW $=2^{\mathrm{N}_{\text {DTC }}-3}$ can 


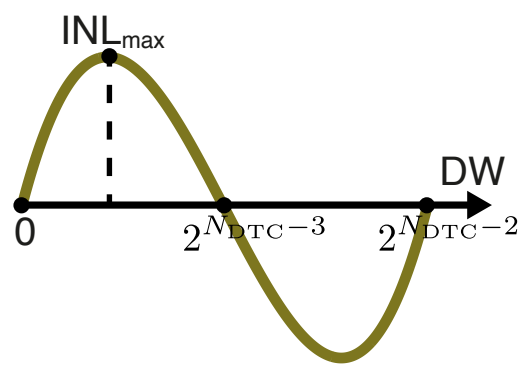

(a) INL (DW)

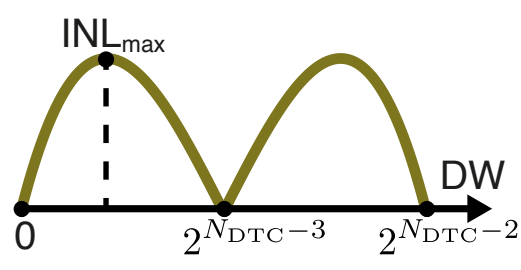

(b) $|\mathrm{INL}(\mathrm{DW})|$

Figure B.2: Cubic INL and its absolute value.

be written as

$$
\begin{aligned}
\mathrm{INL}(\mathrm{DW}) & =\mathrm{DW}\left(\mathrm{DW}-2^{\mathrm{N}_{\mathrm{DTC}}-2}\right) \frac{\mathrm{INL}_{\max }}{\left(2^{\mathrm{N}_{\mathrm{DTC}}-3}\right)^{2}} \\
& +\frac{2}{3} \mathrm{INL}_{\max }
\end{aligned}
$$

And it is shown in Fig. B.1. Its roots are located in

$$
\mathrm{DW}_{01 / 2}=\frac{3 \pm \sqrt{3}}{3} 2^{\mathrm{N}_{\mathrm{DTC}}-3}
$$

Therefore, from (B.28) and (B.23),

$$
\begin{aligned}
& E\{|\operatorname{INL}(\mathrm{DW})|\}=\frac{1}{2^{\mathrm{N}_{\mathrm{DTC}}-2}} \int_{0}^{2^{\mathrm{N}_{\mathrm{DTC}}-2}}|\mathrm{INL}(\mathrm{DW})| \mathrm{dDW} \\
& =\mathrm{INL}_{\max }\left\{\frac{1}{3}\left[2\left(\frac{3-\sqrt{3}}{3}\right)^{3}-1\right]-\left[2\left(\frac{3-\sqrt{3}}{3}\right)^{2}-1\right]\right. \\
& \left.+\frac{2}{3}\left[2 \frac{3-\sqrt{3}}{3}-1\right]\right\} \approx \frac{\mathrm{INL}_{\max }}{4}
\end{aligned}
$$

The same steps can be followed for a generic function INL (DW). The cubic function in Fig. B.2 has the expression:

$$
\begin{aligned}
\mathrm{INL}(\mathrm{DW})= & \mathrm{DW}\left(\mathrm{DW}-2^{\mathrm{N}_{\mathrm{DTC}}-3}\right)\left(\mathrm{DW}-2^{\mathrm{N}_{\mathrm{DTC}}-2}\right) \\
& \frac{\mathrm{INL}_{\max }}{\frac{3-\sqrt{5}}{2}\left(2^{\mathrm{N}_{\mathrm{DTC}}-3}\right)^{3}}
\end{aligned}
$$

By applying in this case the theorem (B.23), it is 


$$
\begin{aligned}
& E\{|\operatorname{INL}(\mathrm{DW})|\}=\frac{1}{2^{\mathrm{N}_{\mathrm{DTC}}-2}} \int_{0}^{2^{\mathrm{N}_{\mathrm{DTC}}-2}}|\mathrm{INL}(\mathrm{DW})| \mathrm{dDW} \\
& \left.=\frac{1}{2^{\mathrm{N}_{\mathrm{DTC}}-2}} \frac{\mathrm{INL}_{\max }}{\frac{3-\sqrt{5}}{2}\left(2^{\mathrm{N}_{\mathrm{DTC}}-3}\right)^{3}} 2 \int_{0}^{2^{\mathrm{N}_{\mathrm{DTC}}-3}} \mathrm{INL}(\mathrm{DW}) \mathrm{dDW}\right] \\
& =\frac{\mathrm{INL}_{\max }}{2(3-\sqrt{5})}
\end{aligned}
$$





\section{Sampled noise power}

\section{C.1 Capacitive DAC}

Fig. C.1 shows the scheme of one matrix of capacitances that can be used to calculate sampled noise power at the matrix output node A. It is derived from Fig. 5.11. Each branch correspond to a weighted capacitance in the matrix. For simplicity a fully binary DAC will be considered. The branch with $\mathrm{C}_{\text {fix }}$ corresponds to the fixed capacitance, showed in gray in Fig. 5.11.

Each inverter connecting a DACcapacitance to the reference voltage or to ground can be modeled as a switch, whose on resistance $R_{\text {on }}$ generates thermal noise that appears at the output $\mathrm{A}$ as $\mathrm{kT} / \mathrm{C}$ noise. Considering the

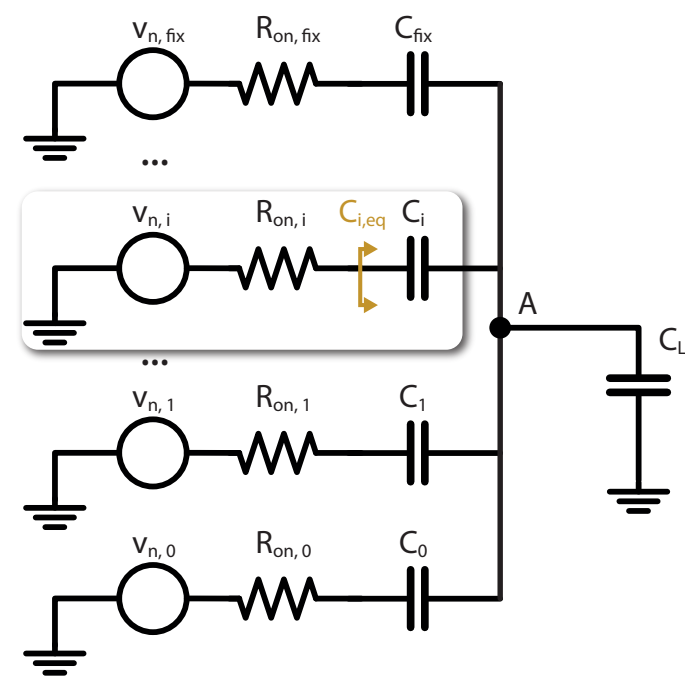

Figure C.1: Scheme of the DAC capacitances to calculate the sampled noise power. 

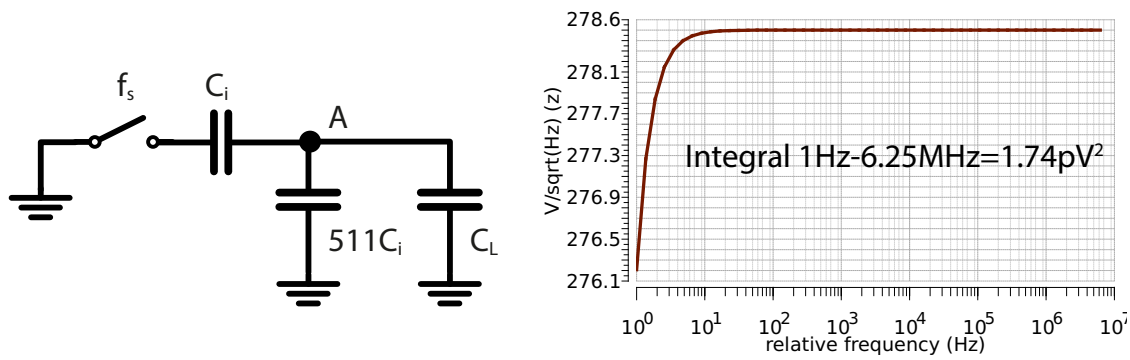

Figure C.2: Noise PSD at node A to verify (C.2). Simulated circuit on the left. Example values for the simulation: $C_{i}=1 \mathrm{fF}, \mathrm{C}_{\mathrm{DAC}}=512 \mathrm{C}_{i}=512 \mathrm{fF}$, $\mathrm{C}_{\mathrm{L}}=1 \mathrm{pF}$. Sample frequency $\mathrm{f}_{\mathrm{s}}=12.5 \mathrm{MHz}$.

$i-$ th branch, the equivalent capacitance seen from $R_{\text {on }}$ is

$$
C_{i, e q}=\frac{C_{i}\left(C_{D A C}-C_{i}+C_{L}\right)}{C_{D A C}+C_{L}}
$$

The sampled noise power on the equivalent capacitance due to the noise generator $v_{n, i}$ transfers to node $A$ according to the capacitance partition

$$
P_{n, i}=\frac{k T}{C_{i, e q}}\left(\frac{C_{i}}{C_{D A C}+C_{L}}\right)^{2}
$$

Where $R_{\text {on }}$ in the other branches has been neglected since it does not affect the final partition but only the transient values. Fig. C.2 shows the noise Power Spectral Density (PSD) at node A, obtained from a Spectre pnoise simulation of one DAC branch. The integral of the PSD until half of the sampling frequency should be equal to (C.2). The simulation result is $1.72 *$ $10^{-12} \mathrm{~V}^{2}$, that agrees with expected noise power from (C.2) $1.8 * 10^{-12} \mathrm{~V}^{2}$. The reason for the $5 \%$ difference is likely due to the simulation accuracy.

The total noise power on node $A$ can be calculated by superposition:

$$
\mathrm{P}_{\mathrm{n}, \mathrm{TOT}}=\sum_{i} \mathrm{P}_{\mathrm{n}, \mathrm{i}}
$$

We can simplify (C.3) in the following way, taking into account the 

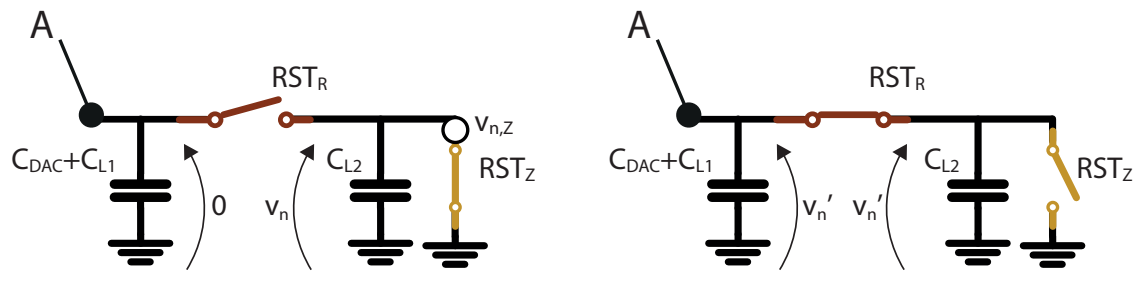

Figure C.3: Charge sharing between node $A$ and one ZCD input.

capacitance weights as in Fig. 5.11

$$
\begin{aligned}
& \mathrm{P}_{\mathrm{n}, \mathrm{TOT}}=\frac{k T}{\left(\mathrm{C}_{\mathrm{DAC}}+\mathrm{C}_{\mathrm{L}}\right)^{2}} \sum_{i} \frac{\mathrm{C}_{i}^{2}}{\mathrm{C}_{\mathrm{i}, \mathrm{eq}}} \\
& =\frac{k T}{C_{D A C}+C_{L}} \sum_{i} \frac{C_{i}}{C_{D A C}-C_{i}+C_{L}} \\
& =\frac{k T}{C_{D A C}+C_{L}}\left[\frac{C_{u}}{C_{D A C}-C_{u}+C_{L}}+\sum_{i=0}^{8} \frac{2^{i} C_{U}}{C_{D A C}-2^{i} C_{u}+C_{L}}\right] \\
& \simeq \frac{k T}{C_{D A C}+C_{L}}\left[\frac{C_{U}}{C_{D A C}+C_{L}}+C_{u} \sum_{i=0}^{8} \frac{2^{i}}{\left(C_{D A C}+C_{L}\right)\left(1-2^{i} \frac{C_{u}}{C_{D A C}+C_{L}}\right)}\right] \\
& \simeq \frac{k T}{C_{D A C}+C_{L}}\left[\frac{C_{U}}{C_{D A C}+C_{L}}+\frac{C_{u}}{C_{D A C}+C_{L}} \sum_{i=0}^{8} 2^{i}\left(1+2^{i} \frac{C_{U}}{C_{D A C}+C_{L}}\right)\right] \\
& =\frac{k T}{\left(C_{D A C}+C_{L}\right)^{2}} C_{U}\left[1+\sum_{i=0}^{8} 2^{i}+\frac{C_{U}}{C_{D A C}+C_{L}} \sum_{i=0}^{8} 2^{2 i}\right] \\
& \simeq \frac{\mathrm{kT}}{\left(\mathrm{C}_{\mathrm{DAC}}+\mathrm{C}_{\mathrm{L}}\right)^{2}} \mathrm{C}_{\mathrm{U}}[1+\frac{511}{2-1}+\underbrace{\frac{1}{512 \cdot 349} \frac{4^{9}-1}{4-1}}_{\approx 0}] \\
& \simeq \frac{k T}{\left(C_{D A C}+C_{L}\right)^{2}} \underbrace{512 C_{u}}_{C_{D A C}}=\frac{k T}{\left(C_{D A C}+C_{L}\right)^{2}} C_{D A C}
\end{aligned}
$$

\section{C.2 Reset switch RST}

The effect on node $A$ due to the noise produced by reset switch $\mathrm{RST}_{z}$ can be evaluated by considering the charge sharing. As shown in Fig. C.3, during the RST state, the noise voltage $v_{n}$ is across $C_{L_{2}}$, while the capacitance $\mathrm{C}_{\mathrm{DAC}}+\mathrm{C}_{\mathrm{L}_{1}}$ is disconnected from it. The total charge is

$$
\left(\mathrm{C}_{\mathrm{DAC}}+\mathrm{C}_{\mathrm{L}_{1}}\right) \cdot 0+\mathrm{C}_{\mathrm{L}_{2}} v_{\mathrm{n}}
$$

In the precharge state the two capacitances are connected together, so 
the total charge is

$$
\left(\mathrm{C}_{\mathrm{DAC}}+\mathrm{C}_{\mathrm{L}_{1}}+\mathrm{C}_{\mathrm{L}_{2}}\right) \cdot v_{\mathrm{n}}^{\prime}
$$

We can write

$$
\mathrm{C}_{\mathrm{L}}=\mathrm{C}_{\mathrm{L}_{1}}+\mathrm{C}_{\mathrm{L}_{2}}
$$

Thus, the voltage ratio is

$$
\frac{v_{n}^{\prime}}{v_{n}}=\frac{C_{L_{2}}}{C_{D A C}+C_{L}}
$$

Therefore, the sampled noise power at node $A$ due to the reset switch $\mathrm{RST}_{z}$ is

$$
\frac{k T}{C_{L_{2}}}\left(\frac{C_{L_{2}}}{C_{D A C}+C_{L}}\right)^{2}=\frac{k T C_{L_{2}}}{\left(C_{D A C}+C_{L}\right)^{2}}
$$




\section{Verilog code for the digital design of the front-end}

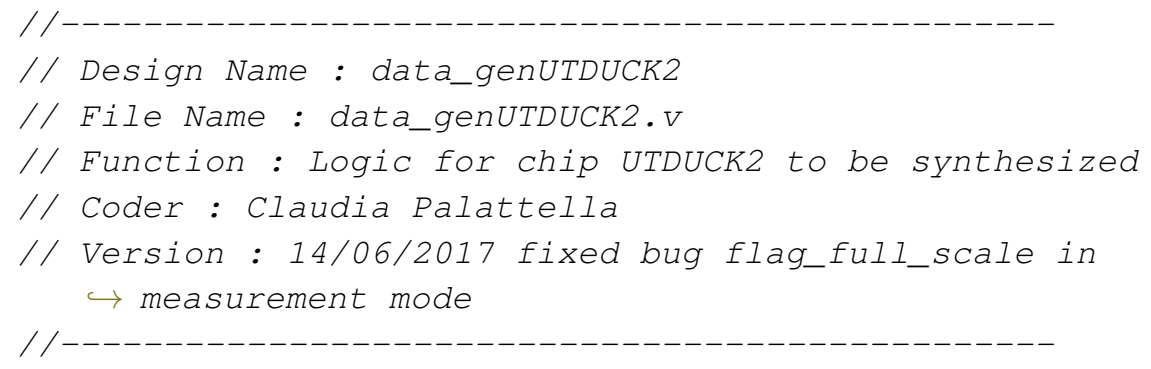


wire [11:0] count, cnt; // internal bus should be $\hookrightarrow$ declared

wire $[11: 0]$ DWFF_1, DWFF_2, DWFF;

wire [1:0] prev_sel_1,prev_sel_2;

wire [21:0] J_step1;

wire [11:0] step1_AR;

wire $[1: 0]$ outA_8U, outB_8U;

wire $[1: 0]$ DWR_8U,DWF_8U, DWRC_8U, DWFC_8U;

wire $[8: 0]$ outAFF, outBFF, outAFF1, outBFF1;

wire $[6: 0] \quad D W R, D W F, D W R C, D W F C$;

wire CK_4_1, CK_4_2 ;

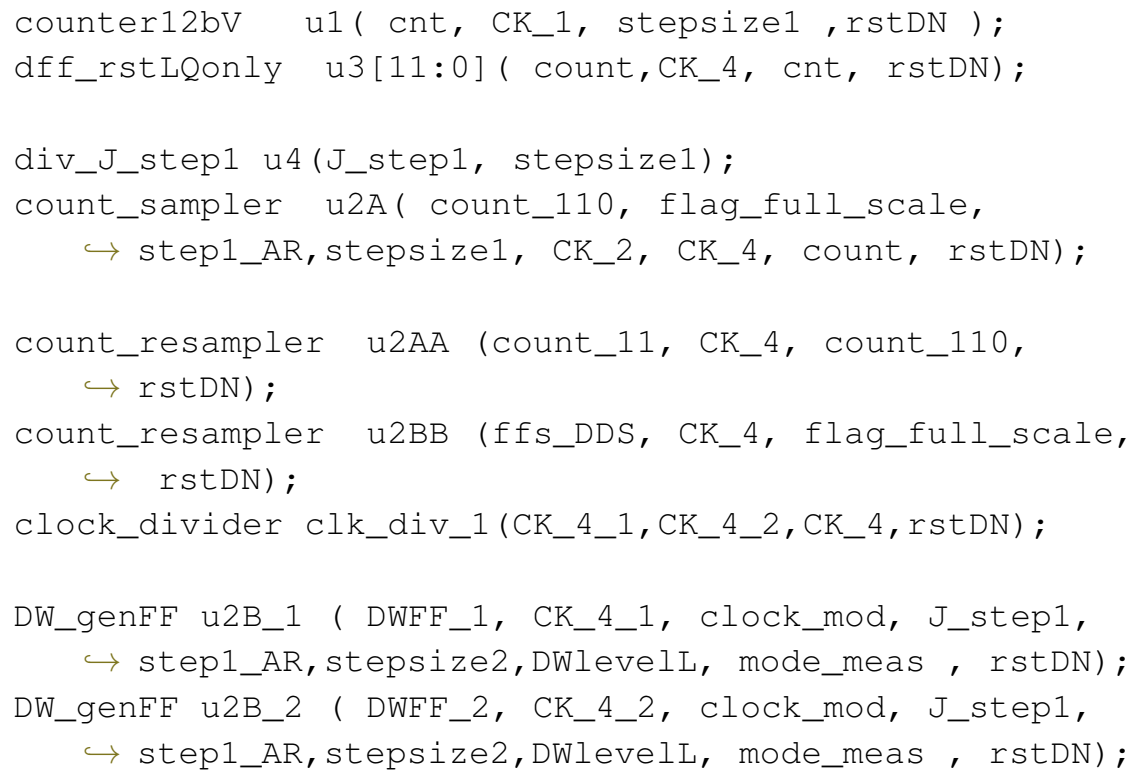

DWFF_MUX mux (DWFF, CK_4, CK_4_1, DWFF_1, DWFF_2) ;

assign DWFF_11=(ffS_DDS \& DWFF[11]);

assign DWNFF_0 $=\sim \mathrm{DWFF}[0]$;

assign prev_sel[1] $=\left(\mathrm{DWFF}[10] \mid \mathrm{DWFF} \_11\right)$;

assign prev_sel[0]=(DWFF[9]|DWFF_11);

dff_rstLQonly u10[8:0] (outAFF, CK_4ext, DWFF [8:0],

$\hookrightarrow$ rstDN);

dff_rstL u10B[8:0] (outAFF1, outBFF1, CK_1, outAFF, rstDN 
data_cross u15 (bu0, CK_4ext,DWNFF_0, DWFF_11, rstDN) ; dff_rstLQonly u15B (bu1, CK_1, bu0, rstDN) ;

dec_bin2un u7 (outA_8U, outA_7U, outAFF1[8:7]) ;

dec_bin2un u8 (outB_8U, outB_7U, outBFF1[8:7]) ;

dff_rstL u11[6:0] (DWR, DWRNout, MSB_resampled_antic,

$\hookrightarrow$ outAFF1[6:0], reset_data_RN); //array of

$\hookrightarrow$ instances

dff_rstL u12[6:0] (DWRC, DWRCNout, MSB_resampled_antic

$\hookrightarrow$, outBFF1[6:0], reset_data_RN); //array of

$\hookrightarrow$ instances

dff_rstL u13[6:0] (DWF, DWFNout, MSB_resampledN_antic,

$\hookrightarrow$ outAFF1[6:0], reset_data_FN); //array of

$\hookrightarrow$ instances

dff_rstL u14[6:0] (DWFC, DWFCNout,

$\hookrightarrow$ MSB_resampledN_antic,outBFF1[6:0], reset_data_FN

$\hookrightarrow$ ); /larray of instances

dff_rstLQonly u15C (DWRCout_0,MSB_resampled_antic, bul,

$\hookrightarrow$ reset_data_RN);

dff_rstLQonly u16C (DWFCout_0,MSB_resampledN_antic,bul,

$\hookrightarrow$ reset_data_FN);

dff_rstL u17[1:0] (DWR_8U, DWRN_8U,

$\hookrightarrow$ MSB_resampled_antic,outA_8U, reset_data_RN);

dff_rstL ul8 (DWR_7U, DWRN_7U, MSB_resampled_antic,

$\hookrightarrow$ outA_7U, reset_data_RN) ;

dff_rstL u19[1:0] (DWF_8U, DWFN_8U,

$\hookrightarrow$ MSB_resampledN_antic,outA_8U, reset_data_FN);

dff_rstL u20 (DWF_7U, DWFN_7U, MSB_resampledN_antic,

$\hookrightarrow$ outA_7U, reset_data_FN);

dff_rstL u21[1:0] (DWRC_8U, DWRCN_8U,

$\hookrightarrow$ MSB_resampled_antic,outB_8U, reset_data_RN);

dff_rstL u22 (DWRC_7U, DWRCN_7U, MSB_resampled_antic,

$\hookrightarrow$ outB_7U, reset_data_RN);

dff_rstL u23[1:0] (DWFC_8U, DWFCN_8U,

$\hookrightarrow$ MSB_resampledN_antic,outB_8U, reset_data_FN) ;

dff_rstL u24 (DWFC_7U, DWFCN_7U, MSB_resampledN_antic, 
$\hookrightarrow$ outB_7U, reset_data_FN) ;

endmodule

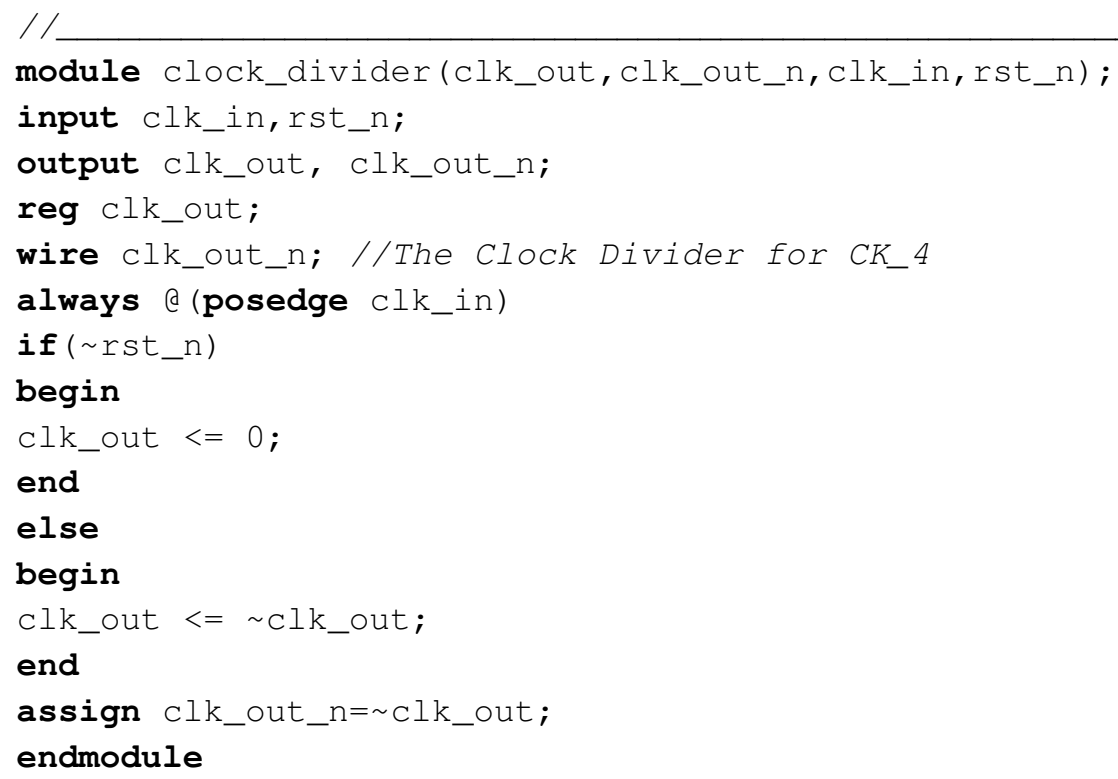


reg $[11: 0]$ out;

always @ (posedge Clk or negedge rstN)

if $(\sim \operatorname{rst})$

out $=0$;

else

out $=($ out + stepsize $) \div(1<<12) ; / /$ modulo $2^{\wedge} 12=4096$

endmodule

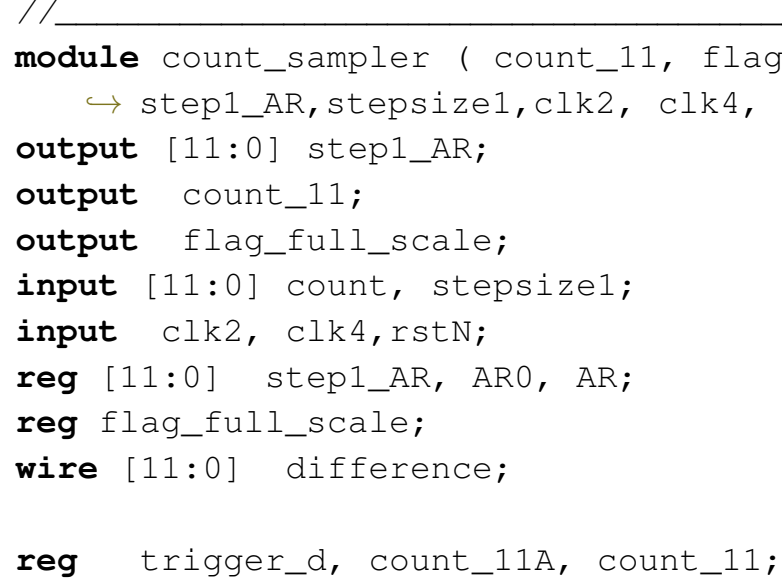

always (posedge clk2) begin

trigger_d $<=$ count [11];

end

//trigger_toggle will have a rising edge every time

$\hookrightarrow$ that count [11] has a rising/falling edge

//Bitwise XOR reduction operator creates pos \& neg

$\hookrightarrow$ edge detect.

wire trigger_toggle;

// assign trigger_toggle = trigger_d^count[11];

xor xorl(trigger_toggle,trigger_d, count[11]);

always (

AR0 [10:0] <=count $[10: 0]$;

$\operatorname{ARO}[11]<=0$;

end

always a (posedge $\mathrm{clk} 4)$ begin 


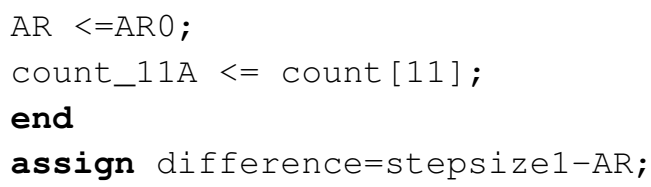




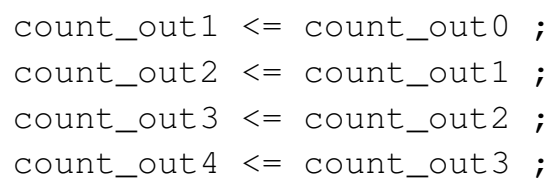

end

end

always a (posedge $\mathrm{clk}$ ) begin

count_out $<=$ count_out 4 ;

end

endmodule

$1 /$

module div_J_step1(J_step1, stepsize1);

output [21:0] J_step1;

input [11:0] stepsize1;

wire [21:0] J_step1;

integer division;

localparam [21:0] $\mathrm{J}=1^{\prime} \mathrm{b} 1<<21 ; / / \mathrm{J}=2 \wedge 11=2048$, shifted $\hookrightarrow$ left 10 positions $=2^{\wedge} 21$

always \&* begin

division=J/stepsize1;

end

assign J_stepl=division;

endmodule

11

module DW_genFF ( DWFF, clk4, clock_mod, J_step1, $\hookrightarrow$ step1_AR, stepsize2,DWlevelL, mode_meas, rstN); output $[11: 0] \mathrm{DWFF}$;

input [21:0] J_step1;

input [11:0] step1_AR;

input [11:0] stepsize2, DWlevelL;

input clock_mod, clk4, mode_meas, rstN;

reg $[11: 0] \quad \mathrm{DWFF}$;

reg $[11: 0]$ DWOFF; 
reg [33:0] pipelined_input;

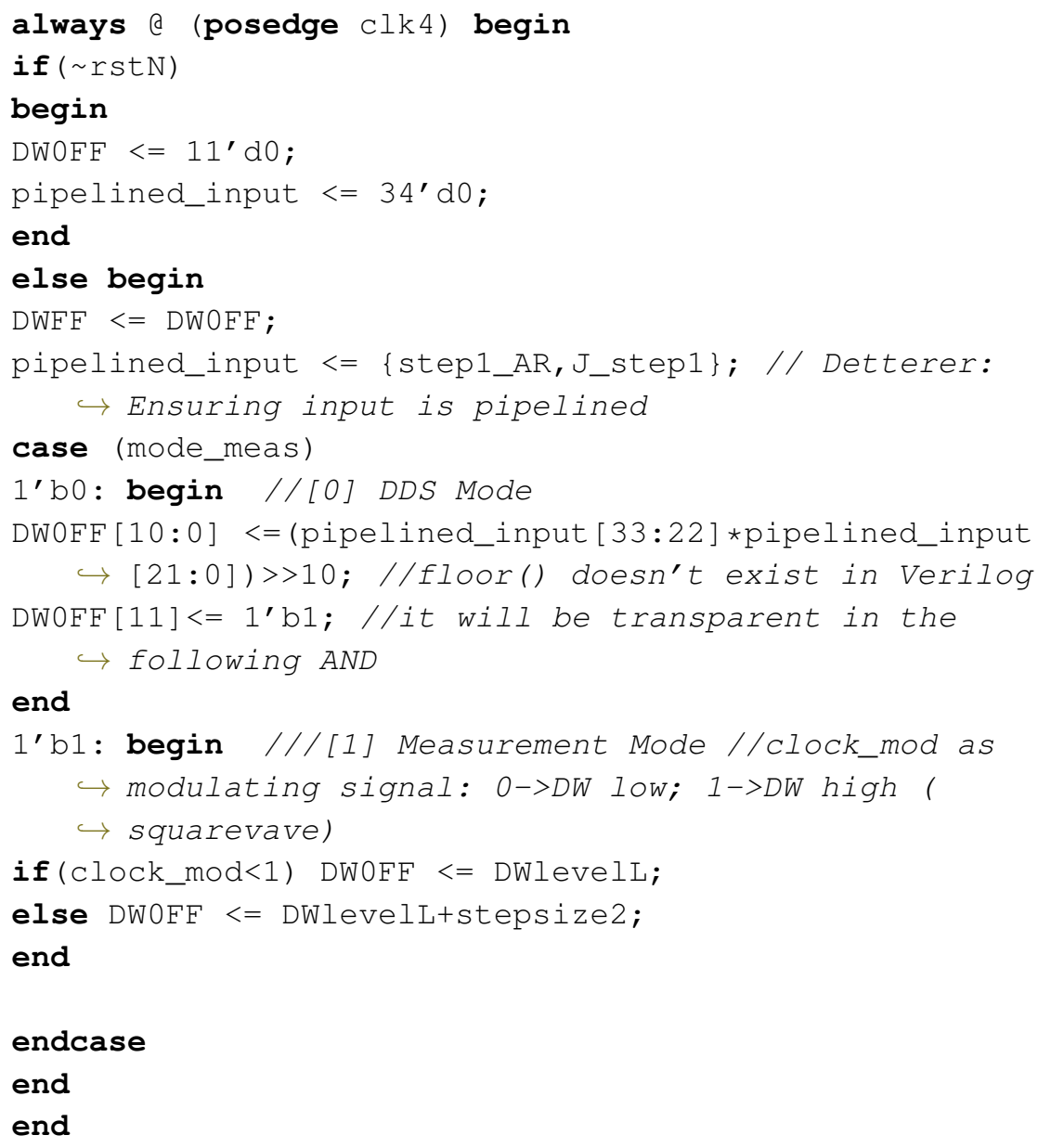


else begin

$q<=d$;

end

end

assign $q \mathrm{q}=\sim \mathrm{q}$;

endmodule

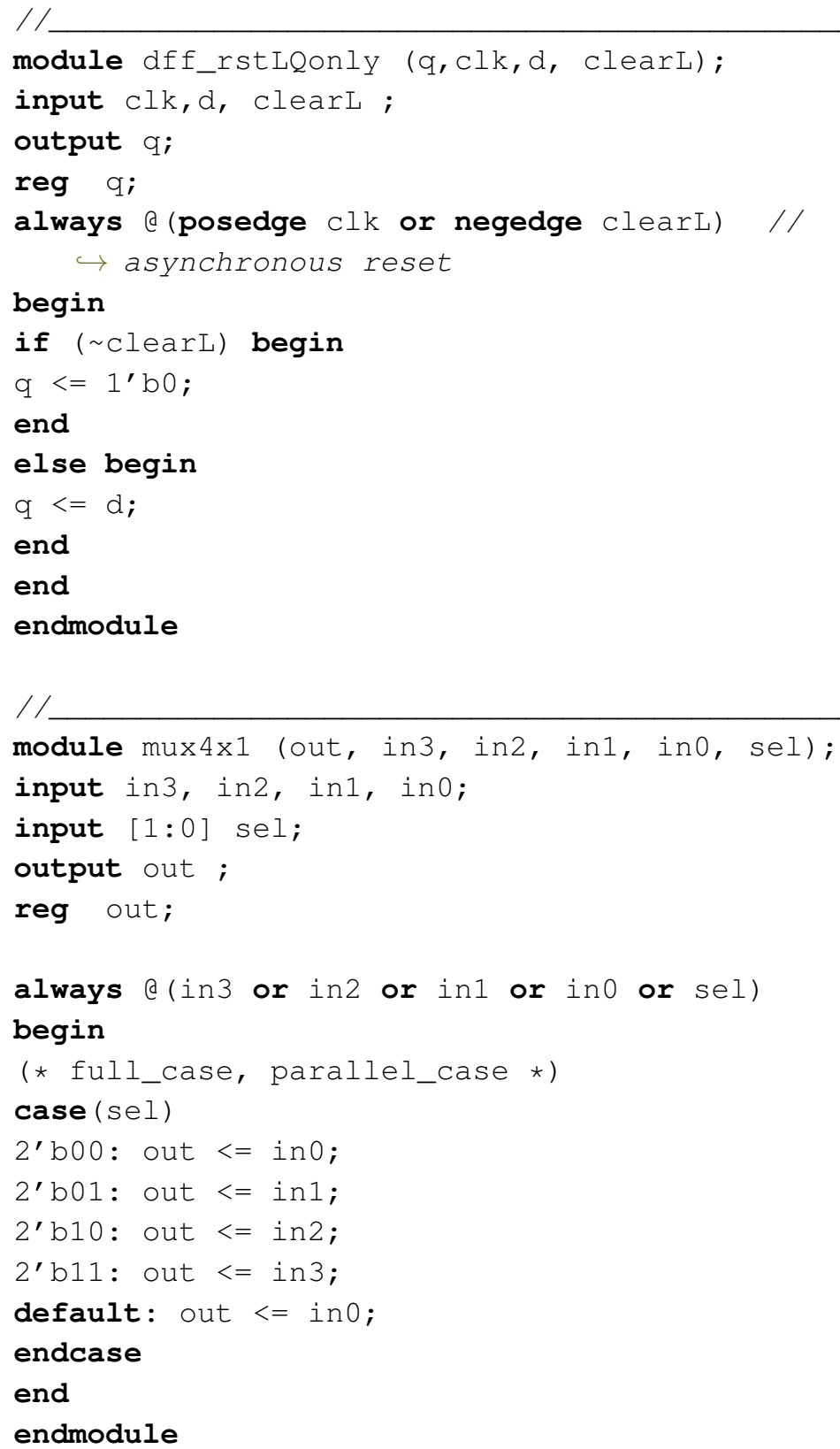




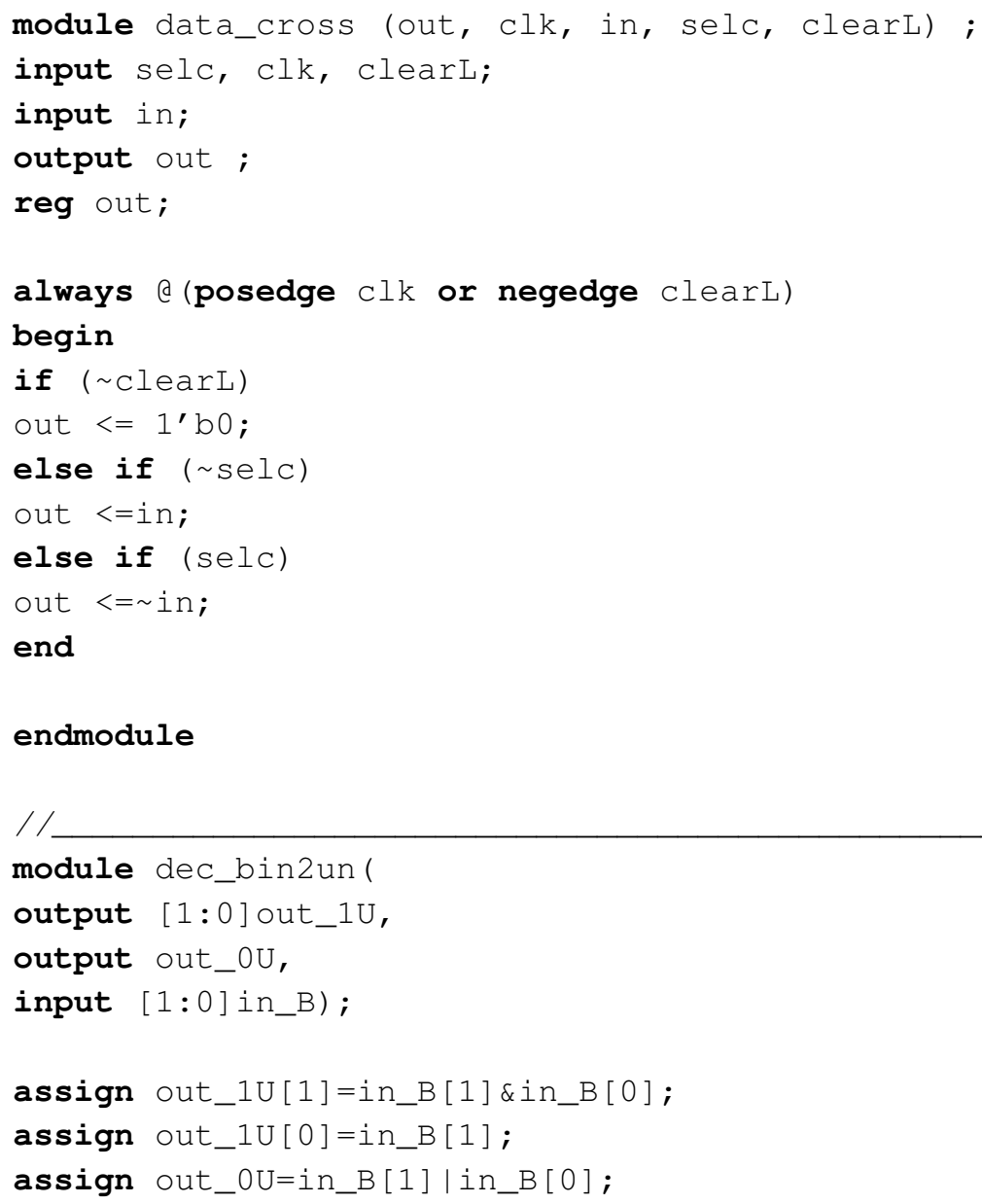




\section{Acknowledgments}

I have been in Twente for more than five years and I have come across many people in this time. We always rely on others to some extent in our life and this is particularly true when you are an expat. Coming alone to Enschede was a challenge for me. I am proud of the way I handled this challenge and am thankful to so many people who contributed to the results I have achieved, not only professionally, but also personally.

Five persons have been constantly present during my PhD journey: Bram Nauta, the head of the ICD group and the first professor I got in contact with, Eric Klumperink, my daily supervisor, Gerdien Lammers, the precious secretary of my group, the technicians Gerard Wienk and Henk de Vries. Bram and Eric have been a point of reference during my studies in Twente. Bram for his charisma and creativity, his capability to identify the key aspects of a problem that brings to effective solutions, his motivational energy. Eric for his analytical curiosity, his practical skills, his empathy and his way of finding positive aspects behind complex situations. I can't thank him enough for his presence during the toughest moments of my $\mathrm{PhD}$ and the precious time he spent with me in the lab. Gerdien has always been an indispensable resource to solve practical problems, handle burocracy, arrange trips or days out, managing the professors' agendas. She was the first person from ICD group that I met, the day of my interview in Twente. During my very first day as employee at the University, she showed me my spot and she welcomed me with a pack of tissues on my desk. I cannot do without them ever since. Gerard has always been the technical problem solver of the group: any issue with software I had, he knew the solution. PhD students pass by, but he stays, keeping the practical know-how within the group. He is indispensable for a successful tape-out. He's also a de facto guardian of the kitchen corner, controlling the Dutch coffee machine and rebuking whoever is against kitchen cleanliness. You can only like someone like him. Henk was a great resource to pick the right equipment for my needs and help to carry such heavy devices. He introduced me to the lab and helped me a lot with spot and equipment reservation. He also used to treat me during the chip measurements, by lending me the best set of tweezers he had, securely stored in his personal drawer... A true gentleman!

As for the other current professors, Harijot was a PhD student during my time in Twente. He has always been very helpful during my design 
reviews and could always suggest a reference in literature I was not aware of. I collaborated with Anne-Johan for the teaching activity. As for Ronan and Frank, I remember their clever contributions during the chiptalks. The discussions with Mark have been crucial to successfully complete my TCAS-I paper. His rigorous yet intuitive perspective has been so inspiring. Thank you all.

I am grateful to everyone I worked and talked with during my PhD, from the first colleagues I met at the University of Twente to the ones that have become true friends and I am still in contact with. The core group of PhD students that I found when I arrived in Twente for the first time was so helpful to get introduced to Dutch culture. Jasper, Mark (now a professor of the ICD group), Michiel, "little" Bram (to distinguish him from the boss), Erik. All of them incredibly sharp. I remember, only a few weeks after my arrival, our Christmas group dinner (meticulously organized by Gerdien) at a brewery, where I learned how beer tradition started as a means to survive from non-potable water several centuries ago. I also learned that Dutch guys drink a lot of beer and stay relatively sober (Jasper was the best at it). I had great discussions with Jasper and Mark when I was struggling with the first measurements on Zhiyu Ru's chip. Having Skype meetings with Zhiyu was also an honor for me. I learned a lot from this first collaboration with a researcher located overseas. Remko, Harish were also there when I arrived and I had great discussions with them. I still keep some extra discrete inverters that Harish gifted me to help me with my measurement setup. Maurizio Burla (from the Telecommunication group) was introduced to me during my first visit in Twente for my interview. He gave me so many practical details about life in Enschede that simplified a lot my settling. Giulia Piccolo (from the Semiconductor group) was the first Italian girl I met at the University. She could understand my little cultural shock and she gave me precious tips for a successful work.

Hugo, Dirk-Jan, Ines, Vishal are the ones I have shared the longest time with. We traveled together, helped each other. We did some teaching activity together. Ines has always been so kind and emphatic, it was a pleasure talking with her about work and also personal things. All of them gave me a valuable support during my research. I enjoyed talking with Johan, Y.C., Ali, Chris, Bart, Alexander, Anoop, Maikel, Joeri, and Labrinus. Thank you, guys!

Finally, the colleagues in my room, the international room of the ICD group. I have learned so much by working close with smart people from all over the world, broadening my perspective in such a multicultural environment. I have already mentioned Harijot and Vishal. We shared so many experiences together. I would also like to thank Ramen, Shadi, Dawei, Zhiliang, Sajad for the several interesting discussions we have had. A special mention to the colleagues in the spot in front of me: Saifullah and Vijaya. Two of the friendliest persons I have ever met. I have shared with Saifullah successful moments and failures, he has always given me 
hope and a boost to my motivation. His help and availability during my final move to Italy have been so meaningful for me. We share the same birth year and similar experiences that keep us connected also presently. Vijaya has always brought positive energy in our room, a good company for hanging out after work, but he is also very focused on work before a deadline. I remember his joy when we got a white board in out room, he was so happy to be able do draw his ideas and questions on the board.

Speaking about closer friend I cannot help mentioning Maurits and Satadal, from the Semiconductor group. They are very curious scientists, attracted by everything related to physics. But they also are true friends of mine. I missed the board games we used to have almost weekly a Maurits' place together with Vishal and Vijaya. So much fun and relaxed time together!

My heartfelt thanks to my parents and to Maurizio. They have been wonderful during the toughest days of my $\mathrm{PhD}$ journey, giving me all the moral support I needed. This thesis is dedicated to my parents, for their endless love and their constant commitment to my personal growth.

Thanks everyone,

Claudia 



\section{Bibliography}

[1] J. Tierney, C. Rader, and B. Gold, "A digital frequency synthesizer," IEEE Transactions on Audio and Electroacoustics, vol. 19, no. 1, pp. 48-57, March 1971.

[2] J. A. Webb, “Digital Signal Generator Synthesizer,” U.S. Patent 3654 450, Apr. 4, 1972.

[3] G. D. Skingle, “Frequency Synthesizer," U.S. Patent 3772 681, Nov. 13, 1973.

[4] C. E. Wheatley, "Digital Frequency Synthesizer With Random Jittering For Reducing Discrete Spectral Spurs," U.S. Patent 4410 954, Oct. 18, 1983.

[5] V. Reinhardt, “Direct digital synthesizers," Dec. 1985.

[6] V. N. Kochemasov and A. N. Fadeev, "Digital-computer synthesizers of two-level signals with phaseerror compensation," Telecommun. Radio Eng., vol. 36/37, p. 55âĂŞ598, October 1982.

[7] P. P. Sotiriadis and K. Galanopoulos, "Direct All-Digital Frequency Synthesis Techniques, Spurs Suppression, and Deterministic Jitter Correction," IEEE Transactions on Circuits and Systems I: Regular Papers, vol. 59, no. 5, pp. 958-968, May 2012.

[8] Cisco, “Cisco Visual Networking Index: Forecast and Trends, 2017 - 2022,” Tech. Rep.

[9] Portable Electronics Market Research Report - Global Forecast 2023. (Last access 2020-6-17). [Online]. Available: https:// www.marketresearchfuture.com/reports/portable-electronics-market-4126

[10] J.-M. Pierson, Large-Scale Distributed Systems and Energy Efficiency: A Holistic View. Wiley, 2015.

[11] F. M. Gardner, Phaselock Techniques, 3rd ed. Wiley-Interscience, 2005.

[12] K. Shu and E. Sanchez-Sinencio, Eds., CMOS PLL Synthesizers: Analysis and Design. Springer, 2005.

[13] D. D. Caro, N. Petra, and A. G. M. Strollo, “Direct Digital Frequency Synthesizer Using Nonuniform Piecewise-Linear Approximation," IEEE Transactions on Circuits and Systems I: Regular Papers, vol. 58, no. 10, pp. 2409-2419, Oct 2011.

[14] T. Yoo, H. C. Yeoh, Y. H. Jung, S. J. Cho, Y. S. Kim, S. M. Kang, and K. H. Baek, “A 2 GHz 130 mW Direct-Digital Frequency Synthesizer With a Nonlinear DAC in $55 \mathrm{~nm}$ CMOS," IEEE Journal of SolidState Circuits, vol. 49, no. 12, pp. 2976-2989, Dec 2014.

[15] J. Vankka and K. A. Halonen, Direct Digital Synthesizers: Theory, Design and Applications, 1st ed. Springer US, 2001.

[16] D. Brandon and K. Gentile. DDS-Based Clock Jitter Performance vs. DAC Reconstruction Filter Performance. Analog Devices. (Last access 2020-6-17). [Online]. Available: https:/ /www.analog.com/ media/en/technical-documentation/application-notes/351016224AN_837.pdf

[17] G. A. Zimmerman and M. J. Flanagan, "Spur-reduced numerically-controlled oscillator for digital receivers," in [1992] Conference Record of the Twenty-Sixth Asilomar Conference on Signals, Systems Computers, Oct 1992, pp. 517-520 vol.1.

[18] Robert P. Gilmore, “Direct digital synthesizer driven phase lock loop frequency synthesizer with clean up phase lock loop," U.S. Patent 5757239 , May 26, 1998.

[19] Earnest R. Jr. Harrison, "Means for Reducing Spurious Frequencies in a Direct Frequency Synthesizer," U.S. Patent 4 185247, Jan 22, 1980.

[20] Robertus L. Van Der Valk, Robertus J. Dequesnoy, Johannes H. A. De Rijk and Menno T. Spijker , "Frequency synthesizer," U.S. Patent 5905388 , May 18, 1999.

[21] H. Nosaka, T. Nakagawa, and A. Yamagishi, "A Phase Interpolation Direct Digital Synthesizer With A Digitally Controlled Delay Generator," in Symposium 1997 on VLSI Circuits, June 1997, pp. 75-76.

[22] H. Nosaka, Y. Yamaguchi, A. Yamagishi, H. Fukuyama, and M. Muraguchi, “A low-power direct digital synthesizer using a self-adjusting phase-interpolation technique," IEEE Journal of Solid-State Circuits, vol. 36, no. 8, pp. 1281-1285, Aug 2001.

[23] T. Finateu, I. Miro-Panades, F. Boissieres, J. B. Begueret, Y. Deval, D. Belot, and F. Badets, “A 500-MHz $\Sigma \Delta$ phase-interpolation direct digital synthesizer," in 2007 IEEE Asian Solid-State Circuits Conference, Nov 2007, pp. 452-455. 
[24] A. Yamagishi, H. Nosaka, M. Muraguchi, and T. Tsukahara, “A phase-interpolation direct digital synthesizer with an adaptive integrator," IEEE Transactions on Microwave Theory and Techniques, vol. 48, no. 6, pp. 905-909, Jun 2000.

[25] L. Dartois and A. Roullet and R. Riboni, "High Frequency Digital Synthesizer With Aperiodic Correction Optimizing The Spectral Purity," U.S. Patent 4792 914, Dec. 20, 1998.

[26] A. C. J. M. Rabaey and B. Nikolic, Digital Integrated Circuits, 2nd ed. Prentice-Hall, 2003.

[27] Hans Tucholski, “Direct digital synthesizer with output signal jitter reduction," U.S. Patent 7103622 , Sep. 5, 2006.

[28] A. Elkholy, S. Saxena, G. Shu, A. Elshazly, and P. K. Hanumolu, “Low-Jitter Multi-Output All-Digital Clock Generator Using DTC-Based Open Loop Fractional Dividers," IEEE Journal of Solid-State Circuits, vol. 53, no. 6, pp. 1806-1817, June 2018.

[29] S. Agarwal and X. Chen, "Phase error correction circuit for a high speed frequency synthesizer," U.S. Patent 7205798 , Apr. 17, 2007.

[30] P. P. Sotiriadis, “Theory of Flying-Adder Frequency Synthesizers-Part I: Modeling, Signals' Periods and Output Average Frequency," IEEE Transactions on Circuits and Systems I: Regular Papers, vol. 57, no. 8, pp. 1935-1948, 2010.

[31] P. P. Sotiriadis, “Theory of Flying-Adder Frequency Synthesizers-Part II: Time- and Frequency-Domain Properties of the Output Signal," IEEE Transactions on Circuits and Systems I: Regular Papers, vol. 57, no. 8, pp. 1949-1963, 2010.

[32] D. Tasca, M. Zanuso, G. Marzin, S. Levantino, C. Samori, and A. Lacaita, “A 2.9-to-4.0-GHz FractionalN Digital PLL With Bang-Bang Phase Detector and 560fs rms Integrated Jitter at 4.5-mW Power," SolidState Circuits, IEEE Journal of, vol. 46, no. 12, pp. 2745-2758, Dec 2011.

[33] N. Markulic, K. Raczkowski, E. Martens, P. E. P. Filho, B. Hershberg, P. Wambacq, and J. Craninckx, “A DTC-Based Subsampling PLL Capable of Self-Calibrated Fractional Synthesis and Two-Point Modulation," IEEE Journal of Solid-State Circuits, vol. PP, no. 99, pp. 1-15, 2016.

[34] H. Liu, D. Tang, Z. Sun, W. Deng, H. C. Ngo, K. Okada, and A. Matsuzawa, “A 0.98mW Fractional-N ADPLL Using 10b Isolated Constant-Slope DTC with FOM of -246dB for IoT Applications in 65nm CMOS," in 2018 IEEE International Solid-State Circuits Conference (ISSCC), Feb 2018.

[35] T. Seong and Y. Lee and S. Yoo and J. Choi, "A -242dB FOM and -75dBc-reference-spur ring-DCO-based all-digital PLL using a fast phase-error correction technique and a low-power optimal-threshold TDC," in 2018 IEEE International Solid - State Circuits Conference - (ISSCC), Feb 2018, pp. 396-398.

[36] N. Markulic, P. T. Renukaswamy, E. Martens, B. van Liempd, P. Wambacq, and J. Craninckx, “A 5.5GHz Background-Calibrated Subsampling Polar Transmitter With -41.3-dB EVM at 1024 QAM in 28nm CMOS," IEEE Journal of Solid-State Circuits, pp. 1-15, 2019.

[37] N. Yan, L. Ma, Y. Xu, S. Chen, X. Liu, J. Xiang, and H. Min, “A Low Power All-Digital PLL With -40dBc In-Band Fractional Spur Suppression for NB-IoT Applications," IEEE Access, vol. 7, pp. 7897-7904, 2019.

[38] S. H. Jung, J. O. Ha, H. J. Yoo, C. W. Cheong, Y. S. Eo, Y. Chun, and W. Kim, “A 3-5 GHz fully integrated CMOS UWB radar chip," in 2013 European Radar Conference, Oct 2013, pp. 57-60.

[39] S. H. Jung and S. G. Kim and W. S. Choi and J. Y. Jeon and J. G. Kim and Y. S. Eo and H. C. Park and H. G. Kim, “A CMOS UWB radar sensor for high speed moving objects," in 2016 46th European Microwave Conference (EuMC), Oct 2016, pp. 1489-1492.

[40] S. Tseng, H. Chou, B. Hu, Y. Kao, Y. Huang, and T. Chu, “Equivalent-Time Direct-Sampling ImpulseRadio Radar With Rotatable Cyclic Vernier Digital-to-Time Converter for Wireless Sensor Network Localization," IEEE Transactions on Microwave Theory and Techniques, vol. 66, no. 1, pp. 485-508, Jan 2018.

[41] D. Zito and D. Pepe and M. Mincica and F. Zito, “A 90nm CMOS SoC UWB pulse radar for respiratory rate monitoring," in 2011 IEEE International Solid-State Circuits Conference, Feb 2011, pp. 40-41.

[42] S. Talwalkar, T. Gradishar, B. Stengel, G. Cafaro, and G. Nagaraj, “Controlled dither in 90 nm digital to time conversion based direct digital synthesizer for spur mitigation," in Radio Frequency Integrated Circuits Symposium (RFIC), 2010 IEEE, May 2010, pp. 549-552.

[43] E. McCune, "Time-filtered squarewave output from direct digital synthesis," in Microwave Symposium Digest (MTT), 2010 IEEE MTT-S International, May 2010, pp. 996-999.

[44] S. A. Talwalkar, "Quantization Error Spectra Structure of a DTC Synthesizer via the DFT Axis Scaling Property," IEEE Transactions on Circuits and Systems I: Regular Papers, vol. 59, no. 6, pp. 1242-1250, June 2012.

[45] S. A. Talwalkar, "Digital-to-Time Synthesizers: Separating Delay Line Error Spurs and Quantization Error Spurs," IEEE Transactions on Circuits and Systems I: Regular Papers, vol. 60, no. 10, pp. 2597-2605, Oct 2013.

[46] Rahkonen, Timo and Eksyma, Harri and Mäntyniemi, Antti and Repo, Heikki, "A DDS Synthesizer 
with Digital Time Domain Interpolator," Analog Integrated Circuits and Signal Processing, vol. 27, no. 1, pp. 109-116, Apr 2001.

[47] G. Manganaro and D. M. W. Leenaerts, Advances in Analog and RF IC Design for Wireless Communication Systems. Academic Press, 2013.

[48] S. Levantino, G. Marzin, and C. Samori, "An Adaptive Pre-Distortion Technique to Mitigate the DTC Nonlinearity in Digital PLLs," IEEE Journal of Solid-State Circuits, vol. 49, no. 8, pp. 1762-1772, Aug 2014.

[49] J. A. McNeill and D. S. Ricketts, The Designer's Guide to Jitter in Ring Oscillators. Springer, 2009.

[50] N. Markulic, K. Raczkowski, P. Wambacq, and J. Craninckx, "A 10-bit, 550-fs step Digital-to-Time Converter in 28nm CMOS," in ESSCIRC (ESSCIRC), 2014 Proceedings of the, Sept 2014.

[51] R. Staszewski, K. Waheed, S. Vemulapalli, F. Dulger, J. Wallberg, C.-M. Hung, and O. Eliezer, "Spur-free all-digital PLL in 65nm for mobile phones," in Solid-State Circuits Conference Digest of Technical Papers (ISSCC), 2011 IEEE International, Feb 2011, pp. 52-54.

[52] M. Safi-Harb and G. Roberts, "70-GHz Effective Sampling Time-Base On-Chip Oscilloscope in CMOS," Solid-State Circuits, IEEE Journal of, vol. 42, no. 8, pp. 1743-1757, Aug 2007.

[53] T. Okayasu, M. Suda, K. Yamamoto, S. Kantake, S. Sudou, and D. Watanabe, "1.83ps-Resolution CMOS Dynamic Arbitrary Timing Generator for gt;4GHz ATE Applications," in 2006 IEEE International Solid State Circuits Conference - Digest of Technical Papers, Feb 2006, pp. 2122-2131.

[54] R. J. de Plassche, CMOS Integrated Analog-to-Digital and Digital-to-Analog Converters, 2nd ed. Springer US, 2003.

[55] R. Mita, G. Palumbo, and M. Poli, "Propagation Delay of an RC-Chain With a Ramp Input," IEEE Transactions on Circuits and Systems II: Express Briefs, vol. 54, no. 1, pp. 66-70, Jan 2007.

[56] P. Chen, F. Zhang, Z. Zong, H. Zheng, T. Siriburanon, and R. B. Staszewski, “A 15- $\mu$ W, 103-fs step, 5bit capacitor-DAC-based constant-slope digital-to-time converter in 28nm CMOS," in 2017 IEEE Asian Solid-State Circuits Conference (A-SSCC), Nov 2017, pp. 93-96.

[57] S. Sievert, O. Degani, A. Ben-Bassat, R. Banin, A. Ravi, W. Thomann, B. Klepser, Z. Boos, and D. SchmittLandsiedel, “A 2 GHz 244 fs-Resolution 1.2 ps-Peak-INL Edge Interpolator-Based Digital-to-Time Converter in 28 nm CMOS," IEEE Journal of Solid-State Circuits, vol. 51, no. 12, pp. 2992-3004, Dec 2016.

[58] H. Jiang, Z. Liu, X. Hao, Z. Zhang, Z. Shen, H. Li, J. Liu, and H. Liao, “A 12-bit 2.5 GHz 0.37ps-PeakINL Digital-to-Time Converter with Parasitic-Insensitive Charge-Based Phase Interpolator," in 2018 IEEE International Symposium on Circuits and Systems (ISCAS), May 2018, pp. 1-5.

[59] X. Gao, E. A. M. Klumperink, M. Bohsali, and B. Nauta, “A Low Noise Sub-Sampling PLL in Which Divider Noise is Eliminated and PD/CP Noise is Not Multiplied by $\mathrm{N}^{2}$," IEEE Journal of Solid-State Circuits, vol. 44, no. 12, pp. 3253-3263, Dec 2009.

[60] K. Raczkowski, N. Markulic, B. Hershberg, J. Van Driessche, and J. Craninckx, “A 9.2-12.7 GHz wideband fractional-N subsampling PLL in 28nm CMOS with 280fs RMS jitter," in Radio Frequency Integrated Circuits Symposium, 2014 IEEE, June 2014, pp. 89-92.

[61] A. Elmallah and M. G. Ahmed and A. Elkholy and W. Choi and P. K. Hanumolu, "A 1.6ps peak-INL 5.3ns range two-step digital-to-time converter in 65nm CMOS," in 2018 IEEE Custom Integrated Circuits Conference (CICC), April 2018, pp. 1-4.

[62] G. Roberts and M. Ali-Bakhshian, "A Brief Introduction to Time-to-Digital and Digital-to-Time Converters," Circuits and Systems II: Express Briefs, IEEE Transactions on, vol. 57, no. 3, pp. 153-157, March 2010.

[63] V. Chillara, Y.-H. Liu, B. Wang, A. Ba, M. Vidojkovic, K. Philips, H. de Groot, and R. Staszewski, “9.8 An $860 \mu \mathrm{W}$ 2.1-to-2.7GHz all-digital PLL-based frequency modulator with a DTC-assisted snapshot TDC for WPAN (Bluetooth Smart and ZigBee) applications," in Solid-State Circuits Conference Digest of Technical Papers (ISSCC), 2014 IEEE International, Feb 2014, pp. 172-173.

[64] N. Pavlovic and J. Bergervoet, "A 5.3GHz digital-to-time-converter-based fractional-N all-digital PLL," in Solid-State Circuits Conference Digest of Technical Papers (ISSCC), 2011 IEEE International, Feb 2011, pp. 54-56.

[65] B. B. O’Brien, “Simple Technique for High-Resolution Time-Delay and Group-Velocity Measurements at Radio Frequencies," Instrumentation and Measurement, IEEE Transactions on, vol. 18, no. 3, pp. 160-162, Sept 1969.

[66] X. Zhu, Y. Li, S. Yong, and Z. Zhuang, "A Novel Definition and Measurement Method of Group Delay and Its Application," Instrumentation and Measurement, IEEE Transactions on, vol. 58, no. 1, pp. 229-233, Jan 2009.

[67] “Ininiium Z-Series Oscilloscopes," Keysight. [Online]. Available: http:/ /literature.cdn.keysight.com/ litweb/pdf/5991-3868EN.pdf

[68] N. Andersson and M. Vesterbacka, "A Vernier Time-to-Digital Converter with Delay Latch Chain Architecture," Circuits and Systems II: Express Briefs, IEEE Transactions on, 2014, Accepted for publication. 
[69] K. Kim, W. Yu, and S. Cho, “A 9 bit, 1.12 ps Resolution 2.5 b/Stage Pipelined Time-to-Digital Converter in 65 nm CMOS Using Time-Register," Solid-State Circuits, IEEE Journal of, vol. 49, no. 4, pp. 1007-1016, April 2014.

[70] S. Haykin, Communication Systems, 4th ed. Wiley, 2000.

[71] S. L. J. Gierkink, “Low-Spur, Low-Phase-Noise Clock Multiplier Based on a Combination of PLL and Recirculating DLL With Dual-Pulse Ring Oscillator and Self-Correcting Charge Pump," Solid-State Circuits, IEEE Journal of, vol. 43, no. 12, pp. 2967-2976, Dec 2008.

[72] “N9030a pxa specifications guide," Keysight.

[73] F. Maloberti, Data Converters, 1st ed. Springer, 2007.

[74] M. Pelgrom, Analog-to-Digital Conversion, 3rd ed., editor, Ed. Springer, 2017.

[75] B. Razavi, RF Microelectronics , 2nd ed. Prentice Hall, 2011.

[76] H. Darabi, Integration of Passive RF Front End Components in SoCs, 1st ed. Cambridge University Press, 2013.

[77] H. J. Visser, Array and Phased Array Antenna Basics. Wiley-Blackwell, 2005.

[78] M. Kihara, S. Ono, and P. Eskelinen, Digital Clocks for Synchronization and Communications. Artech House Publishers, 2002.

[79] T. H. Lin, C. C. Chi, W. H. Chiu, and Y. H. Huang, "A Synchronous 50\% Duty-Cycle Clock Generator in 0.35- $\mu \mathrm{m}$ CMOS," IEEE Transactions on Very Large Scale Integration (VLSI) Systems, vol. 19, no. 4, pp. 585-591, April 2011.

[80] C. Shi and E. SÃąnchez-Sinencio, "On-Chip Two-Tone Synthesizer Based on a Mixing-FIR Architecture," IEEE Journal of Solid-State Circuits, vol. 52, no. 8, pp. 2105-2116, Aug 2017.

[81] M. C. M. Soer, E. A. M. Klumperink, D. J. van den Broek, B. Nauta, and F. E. van Vliet, “Beamformer With Constant-Gm Vector Modulators and Its Spatial Intermodulation Distortion," IEEE Journal of SolidState Circuits, vol. 52, no. 3, pp. 735-746, March 2017.

[82] R. Vishnu and A. S. S., "An avoidance technique for mitigating the integer boundary spur problem in a DDS-PLL hybrid frequency synthesizer," in 2015 International Conference on Communications and Signal Processing (ICCSP), April 2015, pp. 0443-0446.

[83] S. Biswas and V. Revathi, "A fast-switching low-spurious 6-18 GHz hybrid frequency synthesizer," in 2015 IEEE MTT-S International Microwave and RF Conference (IMaRC), Dec 2015, pp. 312-315.

[84] “A Technical Tutorial on Digital Signal Synthesis," Analog Device Inc. [Online]. Available: https: //www.analog.com/media/cn/training-seminars/tutorials/450968421DDS_Tutorial_rev12-2-99.pdf

[85] A. F. Molisch, Wireless Communications, 2nd ed. Wiley, 2011.

[86] A. M. Fahim, Clock Generators for SOC Processors. Circuits and Architectures. Springer US, 2005.

[87] F. Kuo, M. Babaie, H. R. Chen, L. Cho, C. Jou, M. Chen, and R. B. Staszewski, “An All-Digital PLL for Cellular Mobile Phones in 28-nm CMOS with -55 dBc Fractional and -91 dBc Reference Spurs," IEEE Transactions on Circuits and Systems I: Regular Papers, vol. 65, no. 11, pp. 3756-3768, Nov 2018.

[88] C. Yao, W. F. Loke, R. Ni, Y. Han, H. Li, K. Godbole, Y. Zuo, S. Ko, N. Kim, S. Han, I. Jo, J. Lee, J. Han, D. Kwon, C. Kim, S. Kim, S. W. Son, and T. B. Cho, "A $14 \mathrm{~nm}$ fractional-N digital PLL with 0.14 ps rms jitter and $-78 \mathrm{dBc}$ fractional spur for cellular RFICs," in 2017 IEEE International Solid-State Circuits Conference (ISSCC), Feb 2017, pp. 422-423.

[89] C. Ho and M. S. Chen, "A fractional-N digital PLL with background-dither-noise-cancellation loop achieving $<-62.5 \mathrm{dBc}$ worst-case near-carrier fractional spurs in 65nm CMOS," in 2018 IEEE International Solid - State Circuits Conference - (ISSCC), Feb 2018, pp. 394-396.

[90] B. W. Garlepp, K. S. Donnelly, J. Kim, P. S. Chau, J. L. Zerbe, C. Huang, C. V. Tran, C. L. Portmann, D. Stark, Y.-F. Chan, T. H. Lee, and M. A. Horowitz, "A portable digital DLL for high-speed CMOS interface circuits," IEEE Journal of Solid-State Circuits, vol. 34, no. 5, pp. 632-644, May 1999.

[91] D. D. Caro, F. Tessitore, G. Vai, N. Imperato, N. Petra, E. Napoli, C. Parrella, and A. G. M. Strollo, “A 3.3 GHz Spread-Spectrum Clock Generator Supporting Discontinuous Frequency Modulations in 28 nm CMOS," IEEE Journal of Solid-State Circuits, vol. 50, no. 9, pp. 2074-2089, Sept 2015.

[92] S. Sievert, O. Degani, A. Ben-Bassat, R. Banin, A. Ravi, B. U. Klepser, Z. Boos, and D. SchmittLandsiedel, “2.9 A 2GHz 244fs-resolution 1.2ps-Peak-INL edge-interpolator-based digital-to-time converter in 28nm CMOS," in 2016 IEEE International Solid-State Circuits Conference (ISSCC), Jan 2016, pp. $52-54$.

[93] P. P. Sotiriadis, "Exact spectrum and time-domain output of flying-adder frequency synthesizers," IEEE Transactions on Ultrasonics, Ferroelectrics, and Frequency Control, vol. 57, no. 9, pp. 1926-1935, September 2010.

[94] H. Mair and L. Xiu, "An architecture of high-performance frequency and phase synthesis," IEEE Journal of Solid-State Circuits, vol. 35, no. 6, pp. 835-846, June 2000.

[95] David E. Bockelman, “Direct Digital Synthesizer Based on Delay Line With Sorted Taps," U.S. Patent 
6510 191, Jan. 21, 2003.

[96] T. Rapinoja, K. Stadius, L. Xu, S. Lindfors, R. Kaunisto, A. Parssinen, and J. Ryynanen, “A Digital Frequency Synthesizer for Cognitive Radio Spectrum Sensing Applications," IEEE Transactions on Microwave Theory and Techniques, vol. 58, no. 5, pp. 1339-1348, May 2010.

[97] C. E. Wheatley and D. E. Phillips, "Spurious Suppression in Direct Digital Synthesizers," in Thirty Fifth Annual Frequency Control Symposium, May 1981, pp. 428-435.

[98] E. McCune, "Digital frequency synthesizer and method with Vernier interpolation," U.S. Patent 5247469 , Sept. 21, 1993.

[99] L. Vercesi, A. Liscidini, and R. Castello, "Two-Dimensions Vernier Time-to-Digital Converter," IEEE Journal of Solid-State Circuits, vol. 45, no. 8, pp. 1504-1512, Aug 2010.

[100] M. S. Yuan, C. C. Li, C. C. Liao, Y. T. Lin, C. H. Chang, and R. B. Staszewski, “A 0.45V sub-mW all-digital PLL in 16nm FinFET for bluetooth low-energy (BLE) modulation and instantaneous channel hopping using $32.768 \mathrm{kHz}$ reference," in 2018 IEEE International Solid - State Circuits Conference - (ISSCC), Feb 2018, pp. 448-450.

[101] X. Gao, O. Burg, H. Wang, W. Wu, C. T. Tu, K. Manetakis, F. Zhang, L. Tee, M. Yayla, S. Xiang, R. Tsang, and L. Lin, "A 2.7-to-4.3GHz, 0.16p s $\mathrm{rms}_{\mathrm{s}}$-jitter, -246.8dB-FOM, digital fractional-N sampling PLL in 28nm CMOS," in 2016 IEEE International Solid-State Circuits Conference (ISSCC), Jan 2016, pp. 174-175.

[102] X. Gao, E. A. M. Klumperink, and B. Nauta, “Advantages of Shift Registers Over DLLs for Flexible Low Jitter Multiphase Clock Generation," IEEE Transactions on Circuits and Systems II: Express Briefs, vol. 55, no. 3, pp. 244-248, March 2008.

[103] S. S. Soliman and M. D. Srinath, Continuous and Discrete Signals and Systems, 2nd ed. Pearson, 1998.

[104] W. L. Briggs and V. E. Henson, The DFT: An Owners' Manual for the Discrete Fourier Transform . SIAM, 1987.

[105] K. Kundert, "Design of Mixed-Signal Systems on Chip," in 35th Design Automation Conference, 1998 [Online]. Available: https://pdfs.semanticscholar.org/596a/0bfd0749f591fd421d12ad64e9fa2632a8d9. pdf

[106] S. M. Louwsma, A. J. M. van Tuijl, M. Vertregt, and B. Nauta, “A 1.35 GS/s, 10 b, 175 mW TimeInterleaved AD Converter in $0.13 \mu \mathrm{m}$ CMOS," IEEE Journal of Solid-State Circuits, vol. 43, no. 4, pp. 778-786, April 2008.

[107] C. Toumazou, J. Hughes, and N. Battersby, Eds., Switched-Currents: An Analogue Technique for Digital Technology. Peter Peregrinus (IEE), 1993.

[108] J. E. Franca and Y. Tsividis, Eds., Design of Analog-Digital VLSI Circuits for Telecommunications and Signal Processing, 2nd ed. Prentice Hall, 1993.

[109] L. Kull, T. Toifl, M. Schmatz, P. A. Francese, C. Menolfi, M. Brändli, M. Kossel, T. Morf, T. M. Andersen, and Y. Leblebici, "A $3.1 \mathrm{~mW} 8 \mathrm{~b} 1.2 \mathrm{GS} / \mathrm{s}$ Single-Channel Asynchronous SAR ADC With Alternate Comparators for Enhanced Speed in 32 nm Digital SOI CMOS," IEEE Journal of Solid-State Circuits, vol. 48, no. 12, pp. 3049-3058, Dec 2013.

[110] C.-C. Liu, Y.-T. Huang, G.-Y. Huang, S.-J. Chang, C.-M. Huang, and C.-H. Huang, “A 6-bit 220-MS/s time-interleaving SAR ADC in $0.18-\mu \mathrm{m}$ digital CMOS process," in VLSI Design, Automation and Test, 2009. VLSI-DAT '09. International Symposium on, April 2009, pp. 215-218.

[111] T. Kunikiyo, K. Mitsui, M. Fujinaga, T. Uchida, and N. Kotani, “Reverse short-channel effect due to lateral diffusion of point-defect induced by source/drain ion implantation," IEEE Transactions on Computer-Aided Design of Integrated Circuits and Systems, vol. 13, no. 4, pp. 507-514, April 1994

[112] S. Levantino, L. Romano, S. Pellerano, C. Samori, and A. L. Lacaita, "Phase noise in digital frequency dividers," IEEE Journal of Solid-State Circuits, vol. 39, no. 5, pp. 775-784, May 2004.

[113] A. Papoulis and S. U. Pillai, Probability, Random Variables and Stochastic Processes, 4th ed. McGraw-Hill, 2002.

[114] N. Da Dalt and A. Sheikholeslami, Understanding Jitter and Phase Noise. Cambridge University Press, 2018.

[115] A. Lacaita, S. Levantino, and C. Samori, Integrated Frequency Synthesizers for Wireless Systems. Cambridge University Press, 2007.

[116] X. Geng, X. Yu, F. F. Dai, J. D. Irwin, and R. C. Jaeger, “An 11-bit 8.6GHz direct digital synthesizer MMIC with 10-bit segmented nonlinear DAC," in ESSCIRC 2008 - 34th European Solid-State Circuits Conference, Sep. 2008, pp. 362-365.

[117] E. W. Weisstein, “Exponential sum formulas.” [Online]. Available: http://mathworld.wolfram.com/ ExponentialSumFormulas.html 



\section{List of publications}

\section{Peer-reviewed}

[CP:1] C. Palattella, E. A. M. Klumperink, J. Z. Ru, and B. Nauta, “A Sensitive Method to Measure the Integral Nonlinearity of a Digital-to-Time Converter Based on Phase Modulation," IEEE Transactions on Circuits and Systems II: Express Briefs, vol. 62, no. 8, pp. 741-745, Aug 2015.

[CP:2] J. Z. Ru, C. Palattella, P. Geraedts, E. Klumperink, and B. Nauta, “A High-Linearity Digitalto-Time Converter Technique: Constant-Slope Charging," IEEE Journal of Solid-State Circuits, vol. 50, no. 6, pp. 1412-1423, June 2015.

[CP:3] C. Palattella, E. A. M. Klumperink, M. S. O. Alink, and B. Nauta, “Digital-to-Frequency Converters With a DTC: Theoretical Analysis of the Output SFDR," IEEE Transactions on Circuits and Systems I: Regular Papers, pp. 1-14, 2019. 